Clemson University

TigerPrints

August 2020

\title{
Functional Description for Thick Bistable Carbon Fiber Laminates with Rayleigh-Ritz, Abaqus, and Experiments
}

Christopher Henry Knippenberg

Clemson University, christopher.knippenberg@gmail.com

Follow this and additional works at: https://tigerprints.clemson.edu/all_theses

\section{Recommended Citation}

Knippenberg, Christopher Henry, "Functional Description for Thick Bistable Carbon Fiber Laminates with Rayleigh-Ritz, Abaqus, and Experiments" (2020). All Theses. 3407.

https://tigerprints.clemson.edu/all_theses/3407

This Thesis is brought to you for free and open access by the Theses at TigerPrints. It has been accepted for inclusion in All Theses by an authorized administrator of TigerPrints. For more information, please contact kokeefe@clemson.edu. 


\section{Functional Description for Thick Bistable Carbon Fiber Laminates With Rayleigh-Ritz, Abaqus, and Experiments}

\begin{tabular}{c} 
A Thesis \\
Presented to \\
the Graduate School of \\
Clemson University \\
\hline In Partial Fulfillment \\
of the Requirements for the Degree \\
Master of Science \\
Mechanical Engineering \\
Christopher H. Knippenberg \\
August 2020 \\
\\
Dr. Oliver J. Myers, Committee Chair \\
Dr. Garrett Pataky \\
Dr. Suyi Li \\
\end{tabular}




\section{Abstract}

Composite laminates constructed in an asymmetric/unsymmetric cross-ply layup orientation exhibit two stable static equilibrium positions and may be actuated to snap from a primary stable cure shape to an inversely related secondary stable cure shape. This study aims to add functional descriptions of thick square bistable laminates, whose increased thickness risk the loss of bistability, through previously established analytical and finite element analysis (FEA) approaches as well as experimentation. Rayleigh-Ritz and Abaqus CAE 6.14 FEA software were both used to determine the cure shapes of carbon fiber reinforced polymer (CFRP) laminates composed of DA409 and TR50S-12k carbon fibers. These laminates were modeled to act as square thick bistable composites with sidelengths up to $0.914 \mathrm{~m}$. Visualizations of the out-of-plane displacements are shown for both methods with descriptions of each approach. Experiments using DA409/8552 and TR50S12K/Newport 301 prepreg are used to further describe and develop the fundamental description for thick bistable laminates in terms of loss of bistability, deflection, curvature, actuation load, and shape.

The analytical model is an extension of Hyer's (2002) and Mattioni's (2009) work applied to thick bistable laminates where the primary assumption was the $\mathrm{x}$-axis curvature equaled the negative $y$-axis curvature for the primary and secondary stable positions, respectively. This assumption leads to the already cemented conclusion that bistable laminates, once cured, take on one of two equal, yet inversely related, paraboloid shapes. Fourth order polynomials were used to describe the curvature along the principle laminate axes, differing from the previously used Menger curvatures, (three-point approximation). Bifurcation plots using peak deflections and average curvature generated from Rayleigh-Ritz, FEA simulations, and experiments clearly showed bistability existed to approximately 30 plies; however, FEA showed predictions upwards of 70 plies. On the other hand, energy landscapes generated from FEA indicated a significant degradation of bistability starting at 
36 plies. Experimentation was performed on a test stand mimicking the same boundary conditions used in FEA while applying a centroidal out-of-plane transverse load. Experimental observations showed decreased peak displacements of stable cure shapes in addition to indications that the $\mathrm{x}$ axis curvature had a significant difference in magnitude compared to the negative y-axis curvature. However, the existence of bistability agreed closely with Rayleigh-Ritz and FEM energy landscape plots, with clear "snaps" ending at thicknesses of 28-36 plies. Moreover, actuation force was found to be significantly similarly during experiments when compared to FEA simulations. Significant differences in curvature predictions and bistability loss from FEA was attributed to the combination of material characteristic differences for DA409/G35 and TR50S-12K, limitations of the experimental setup, and hand layup fabrication errors, curvature calculation methods, and the exclusion of defects in models. Lastly, although this paper raises more questions, it also shows viability of thicker bistable laminates to be used in macroscale applications where shape morphing or shape-retention attributes are a necessary constraint. 


\section{Dedication}

This paper is dedicated to those who remain compassionate in their work towards bettering society, creating progress, and providing long lasting contributions.

To my family.

To my grandfather, Dr. Rodney K. Skogerboe.

To my close friends. 


\section{Acknowledgments}

Thanks would like to be extended to the Clemson University Department of Mechanical Engineering, whose professors and fellow students have guided me in this almost decade long journey. I would also like to extend a very grateful thanks to my family and close friends, including in no particular order Emily E. Johnson, Ian McCraw, David Kim, Christopher Nelon, and Jon Oakley, who supported me during this period, as I could not have completed this work without them. 


\section{Table of Contents}

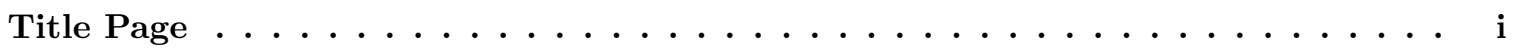

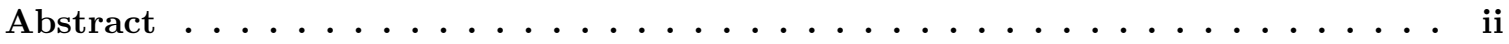

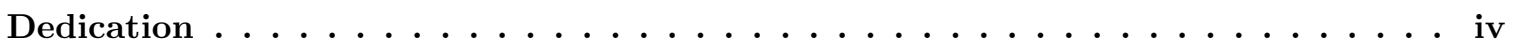

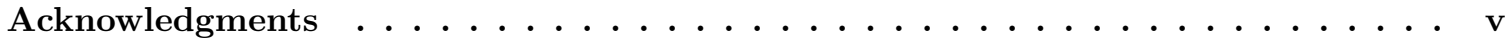

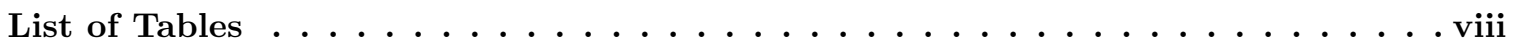

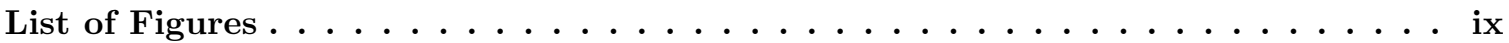

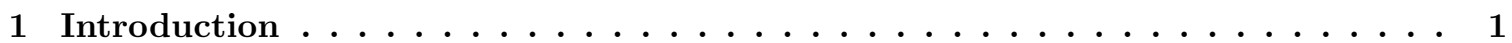

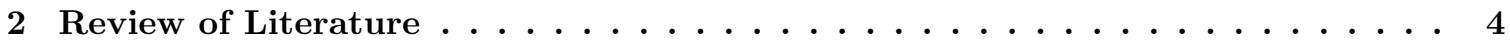

2.1 Mechanics of Composite Materials . . . . . . . . . . . . . . . . . . 4

2.2 Bistable Laminates Cure Shape Prediction . . . . . . . . . . . . . . . . . . . . 15

2.3 Bistable Laminates Static Snapthrough Behavior . . . . . . . . . . . . . . . . . . . 21

2.4 Bistable Laminates Potential Applications . . . . . . . . . . . . . . . . . . . . 22

2.5 Fabrication Techniques for CFRP Structures . . . . . . . . . . . . . . . . 23

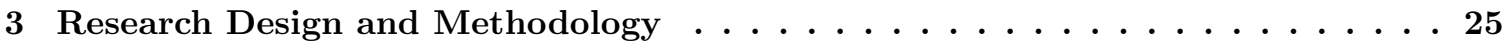

3.1 Purpose of the Study . . . . . . . . . . . . . . . . . . . . . . 25

3.2 Explanation of Research Design . . . . . . . . . . . . . . . . . . . . . 26

3.3 Justification of the Methodological Decisions and Their Limits . . . . . . . . . . 41

3.4 Summary of Methods . . . . . . . . . . . . . . . . . . . . 42

4 Results and Implications $\ldots \ldots \ldots \ldots \ldots \ldots$

4.1 Cure Shape . . . . . . . . . . . . . . . . . . . . . . . . 43

4.2 Snapthrough behavior . . . . . . . . . . . . . . . . . . . . . 63

4.3 Sources of Error . . . . . . . . . . . . . . . . . . . . . 70

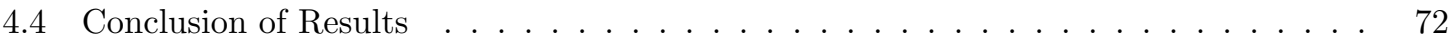

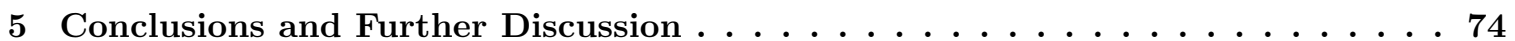

5.1 Answering the Research Question . . . . . . . . . . . . . . . . . . . . . 75

5.2 Theoretical Implications and Recommendations for Further Research . . . . . . . . . 77

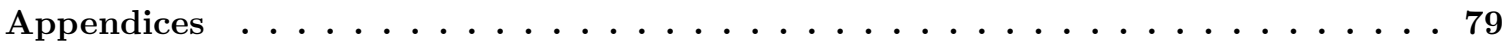

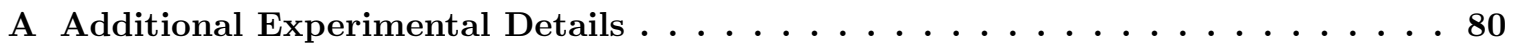

B Selected Raw Data and Images . . . . . . . . . . . . . . 85 


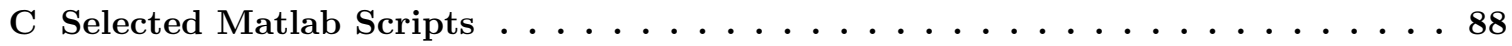

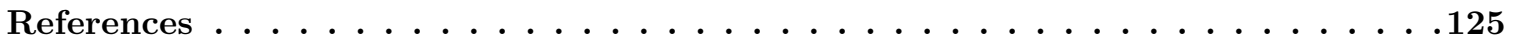




\section{List of Tables}

3.1 Elastic and Flexural Moduli Comparison Table between DA409 and AS4 . . . . . . 26

3.2 Material Properties Used for AS4/8552 and TR50S-12K/NP301. . . . . . . . . . . . 26

3.3 Material Properties Used for AS4/8552 and TR50S-12K/NP301 during FEA. . . . . 33

4.1 Calculated Energy (Joules) of Stable Equilibrium Shapes Generated from Rayleigh-Ritz. 44

4.2 DA409: Coefficients for $P_{4}$ Polynomials Describing Deflection Shape Generated from FEA. . . . . . . . . . . . . . . . . . . . . . . . 52

4.3 TR50S: Coefficients for $P_{4}$ Polynomials Describing Deflection Shape Generated from FEA. . . . . . . . . . . . . . . . . . . . . 52 


\section{List of Figures}

1.1 Tradeoff Between Structural Requirements for Shape Adaptive Structures . . . . . . 2

2.1 Possible Laminate Shapes. . . . . . . . . . . . . . . . . . . . . . . . . . . . . . . . . . 16

2.2 Hyer's Bifurcation Diagram. . . . . . . . . . . . . . . . . . . . . . 17

2.3 Mattioni's Out-of-Plane Displacement Predictions. . . . . . . . . . . . . . . . 20

3.1 Y and -Y FEA Boundary Conditions Highlighted on the Mesh for Determining Snapthrough Actuation Load. . . . . . . . . . . . . . . . . . . . . . 32

3.2 Nodes Contained in Sets are Highlighted in Red Indicating Locations of Loads and Boundary Conditions. . . . . . . . . . . . . . . . . . . 34

3.3 Cooling Boundary Conditions for Initial Step. . . . . . . . . . . . . . . . . . . 35

3.4 FEA Mesh with Highlights of the Loading Area Used for Determining Snapthrough Actuation Loads. . . . . . . . . . . . . . . . . . . . . . . 36

3.5 Vacuum Bag Opened up to Show Layup with Green Peel Ply, Red Peel Ply, Breather, and Transparent Vaccum Bag Sealed with Mastic Tape. . . . . . . . . . . . . . 39

3.6 Simply Supported Loading Condition Experiments. . . . . . . . . . . . . . . . . . . . 40

3.7 Clamped Fixture Support Diagram for Experiments. Eye bolt was the Location of Applied Load. . . . . . . . . . . . . . . . . . . . . . . . 40

4.1 Energy Developed at Equilibrium Stable Configurations for Increasing Thickness . . 44

4.2 Visualizations of Cure Shape Stable Configurations for a 4-Ply DA409 Laminate from Rayleigh-Ritz . . . . . . . . . . . . . . . . . . . . 45

4.3 30-Ply DA409 Rayleigh-Ritz Shape Cure Shape Prediction Using Matlab. Notice the Parabolic Edge Compared to the Flat Edge of Figure 4.2. . . . . . . . . . . . . . . 46

4.4 Saddle Shape Prediction for 36-Ply DA409 Laminate Generated by Rayleigh-Ritz Using Matlab surf. . . . . . . . . . . . . . . . . . . . . . . . 46

4.5 Rayleigh-Ritz Bifurcation of Deflections for DA409. . . . . . . . . . . . . 47

4.6 Rayleigh-Ritz Bifurcation of Deflections for TR50S. . . . . . . . . . . . . . . . . 47

4.7 Rayleigh-Ritz Bifurcation of Curvature for DA409. . . . . . . . . . . . . . . . . . 49

4.8 Rayleigh-Ritz Bifurcation of Curvature for TR50S. . . . . . . . . . . . . . . . . 49

4.9 Post-Cure Visualizations of Stable Shapes for 14-Ply DA409. . . . . . . . . . . . . . 51

4.10 Bifurcation of Deflection for DA409 Generated from FEA . . . . . . . . . . . . . 51

4.11 Bifurcation of Deflection for TR50S Generated from FEA . . . . . . . . . . . . . 52

4.12 Bifurcation of Curvature for DA409 Generated from FEA . . . . . . . . . . . . . . 53

4.13 Bifurcation of Curvature for TR50S Generated from FEA . . . . . . . . . . . . . 54

4.14 Selected Energy Landscapes of TR50S. . . . . . . . . . . . . . . . . . 55

4.15 Selected Nondimensionalized TR50S Energy Landscapes. . . . . . . . . . . . . . . . 55

4.16 Selected Nondimensionalized TR50S Energy Landscapes from $0.8<t<2$. Inset: Figure $4.15 \ldots \ldots \ldots \ldots \ldots \ldots \ldots \ldots$

4.17 Stable Configuration A for a 6-Ply $325 \mathrm{~mm} x$ x 325mm Sample. . . . . . . . . . . . . . 57

4.18 Stable Configuration B for a 6 -Ply $325 \mathrm{~mm} \times 325 \mathrm{~mm}$ Sample. . . . . . . . . . . . . 58 
4.19 Nondimensional DA409 Experimental Deflection Compared to Rayleigh-Ritz and FEA. 59

4.20 Nondimensional TR50S Experimental Deflection Compared to Rayleigh-Ritz and FEA. 59

4.21 Nondimensional DA409 Curvature for Rayleigh-Ritz, FEA, and Experimentation. . . 60

4.22 Nondimensional TR50S Curvature for Rayleigh-Ritz, FEA, and Experimentation. . . 60

4.23 DA409 18-Ply Cure-Shape Side-by-Side Visualization from FEA and Experiments. . 61

4.24 DA409 36-Ply Saddle Shape Unstable Sample. It was Observed that Axis Curvatures were not Equal in Magnitude. . . . . . . . . . . . . . . . . . . . . 61

4.25 Force-Displacement of Centroid for 14-Ply TR50S Laminate. . . . . . . . . . . . . 64

4.26 Snapthrough and Snapback Actuation Load Curves for DA409 and TR50S Predicted by Finite Element Analysis. . . . . . . . . . . . . . . . . . . . . . . . . . 64

4.27 Snapthrough/Snapback Actuation Load Curve for DA409 as a Function of Number

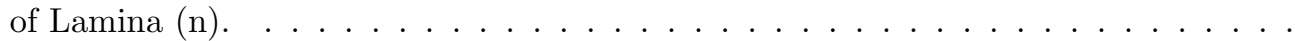

4.28 Snapthrough/Snapback Actuation Load Curve for TR50S as a Function of Number

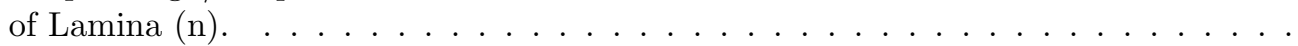

4.29 Nondimensional Snapthrough Actuation Load Curve for DA409 as a Function of Num-

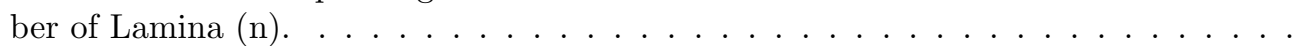

4.30 Nondimensional Snapthrough Actuation Load Curve for TR50S as a Function of Number of Lamina $(\mathrm{n}) . \ldots$. . . . . . . . . . . . . . . . . . . . .

4.31 TR50S 10 Ply Experimental Cure Shape in Clamped Test Stand with Loading Apparatus to Centroid. . . . . . . . . . . . . . . . . . . 68

4.32 TR50S 10-Ply Experimental Intermediate Modal Shape A. . . . . . . . . . . . . . 69

4.33 TR50S 10-Ply Experimental Intermediate Modal Shape B. . . . . . . . . . . . . . . . 69

4.34 TR50S 10-Ply Experimental Intermediate Modal Shape C. . . . . . . . . . . . . . . . 69

4.35 FEA Generated TR50 Linear Perturbation Modal Solutions for a 10-ply Laminate. . 70

4.36 28-Ply TR50S Sample Accidentally Bonded with Mold Plate. . . . . . . . . . . . . 71

4.37 Sample Surface Defects for a DA409 Laminate. . . . . . . . . . . . . . . . . . . 72

4.38 FEA Generated DA409 Bifurcation Plots Examining the Effects of Lamina Thickness on Results. . . . . . . . . . . . . . . . . . . . . . 73

A.1 Stand Used to Increase Consistency of Prepreg Unitape Cutting. . . . . . . . . . . . . . 81

A.2 Typical Vacuum Bag Technique Layup $[40] \ldots \ldots$. . . . . . . . . . . . . . . . 82

A.3 Vacuum Bag Technique Layup Procedure with Green Peel Ply, Red Peel Ply, Breather, and Transparent Vaccum Bag Sealed with Mastic Tape against the Mold. . . . . . . 82

A.4 Inside the Oven. A Hose was Fitted Through the Sidewall to Attach to a Fitting on the Mold Plate. . . . . . . . . . . . . . . . . . . . . . . 83

A.5 Vacuum Bag Pump Used. . . . . . . . . . . . . . . . . . . . . . . . 83

A.6 Toggle Clamp on 80/20 Material Shown Mounted to Bracket. . . . . . . . . . . . . 84

A.7 Toggle Clamp Bracket Designed to Accept 1" Gussets and a McMasterCarr 5128A24 Toggle Clamp. . . . . . . . . . . . . . . . . . . . . . . 84

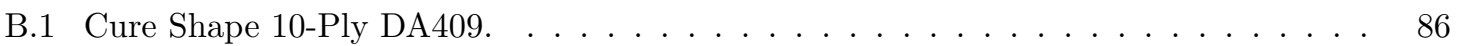

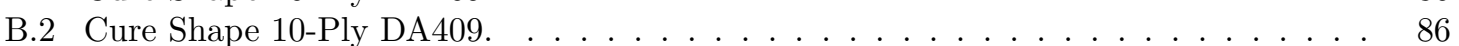

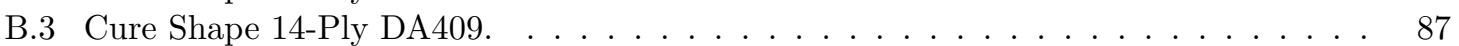

B.4 Cure Shape 14-Ply DA409. . . . . . . . . . . . . . . . . . . . . 87 


\section{Chapter 1}

\section{Introduction}

Materials research is an ongoing field whose desire is to develop new materials and study the behaviors of said materials so that they may be implemented into industry. New materials are continuously being created in order to fit current niche use and also to push future engineering designs. Materials research can be subdivided into two fields: material development and properties and behavior, where material properties drive the behavior of said materials under certain conditions. The design space for a material is dependent on the intended behavior of the material in the conditions dictated by design requirements. Thus, the functionality of a material is a product of its behavior.

A subfield of material development is smart responsive materials that have the inherent property of coupling across physical domains such that the materials provide an adaptive or reactive stance to environmental stimuli $[1,2]$. Examples of materials that exhibit these coupling properties are thermo-mechanical coupling shape memory alloys/polymers, electro-mechanical piezoelectrics, photovoltaics and photomechanical materials, and ferrofluids.

Shape-morphing materials are another unique subfield in that they have the ability to adapt shape to a given environment or situation without the need for external components. This leads to the creation of structures which have the conflicting attributes of load bearing, lightweight, and shape-adaptive [3]. The functionality of shape-morphing structures and materials is ongoing and a desire to find engineering solutions that meet all of these requirements has been a challenge. One such solution has been the creation and study of multistable shape-morphing materials, that can exhibit large displacements without conventional actuator mechanisms. 


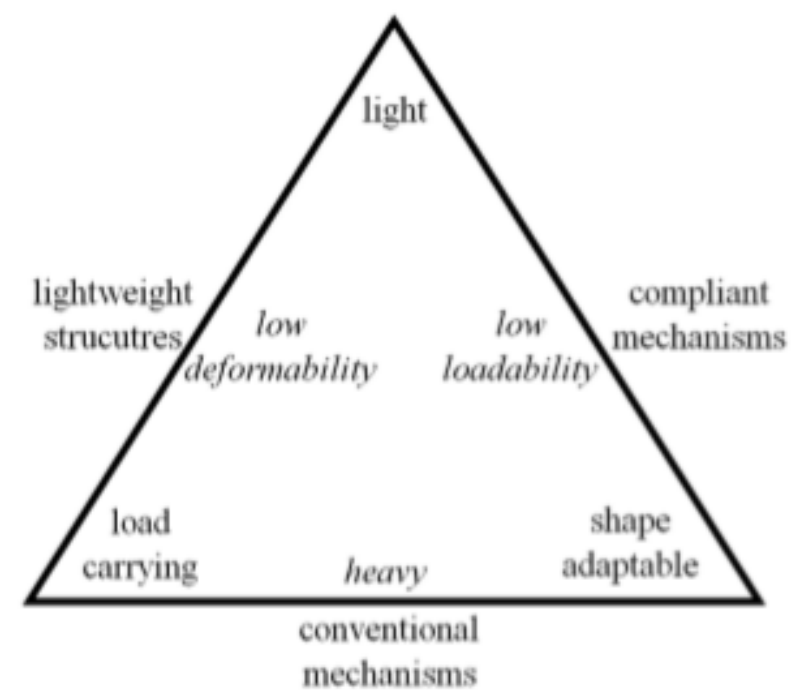

Figure 1.1: Tradeoff Between Structural Requirements for Lightweight Shape Adaptations (With permission to republish from[3]).

Bistable unsymmetric cross-ply laminated composites is one of the most widely studied multistable shape-morphing materials as their incorporation of carbon fiber reinforced polymers and large displacements meets some of the major design challenges while also having the ability to tailor the stiffnesses [3]. Additionally, the natural stability aspects of bistable laminates allows them to maintain their shape without the need for external mechanisms. These laminates are created using the geometric nonlinearities and the mismatched longitudinal and transverse thermal expansion coefficients assembled about the midplane $[4,5]$. When laminates are fabricated this way, upon reaching final post-cure temperature, residual bending and twisting stresses create significant outof-plane deformations in addition to multiple equilibrium configurations $[3,5,6]$. Using external loads and moments it is possible to cause these laminates to actuate snapthrough from one primary equilibrium configuration to a secondary equilibrium configuration $[4,5,7,8]$. The functionality of bistable laminates has not yet been fully developed as two significant cons exist. The first is that the loads to actuate snapthrough are not necessarily high enough to resist snapthrough when desired and second these laminates are highly susceptible to the effects of moisture ingress [3]. Many concepts have been developed to mitigate these downsides such as incorporation of stiffeners,varied layups, and geometries. With this in mind, these bistable laminates still have potential applications in the automotive, aerodynamic, and renewable industries once these challenges are fully addressed. 
In order to meet future industry implementation, this research aims to define the functional design space of thick bistable carbon fiber laminated composites, whose increased thickness risk the loss of bistability, via descriptions of their stable shapes after fabrication and the forces necessary to induce snapthrough. As such, a previously established physics-based analytical method and a finite element methods will be applied to these thick laminated plates along with comparisons to experimental samples will be done. Additionally, studies analyzing the thickness parameter and bistable behavior will be explored from finite element analysis and experimental confirmation. 


\section{Chapter 2}

\section{Review of Literature}

This literature review highlights the relevant mechanics of composite materials and relevant research done on bistable CFRP laminates, the prediction of their post-cure shapes, the study of actuation force to cause snapthrough, and functional applications.

\subsection{Mechanics of Composite Materials}

Composites are materials comprised of two or more constituent materials that tend to have different material properties. Composites take advantage of each individual materials physical or chemical properties and combine them into one to form a new material with new properties. Some motivations to create composites are favorable strength, stiffness, fatigue life, corrosion resistance, weight, or thermal coefficients $[6,9,10]$. Because of this, composites have been widely fielded in the aerospace, aerospace, automotive, and renewable energy sectors $[6,7]$.

Basic composite geometry is simple since the two categories of constituents that make up composite materials are the matrix and reinforcement. The matrix is the binding agent that holds the reinforcement in place. Reinforcements are stronger than the matrix and give composites their desired characteristics while the matrix tends to make the overall composite lighter or cheaper. Matrix materials are typically either polymers, metals, ceramics, or carbon. Furthermore, composites can be broken down into three categories based on their geometries: fiber-based composites, particulate composites, laminated composites, or combination thereof. 


\subsubsection{Composite Laminate Nomenclature and Types}

Composites laminates are made up many layers, or lamina, usually bonded together. These laminates can be described simply by their principle material directions, size, and stacking sequence. The principle material directions of lamina are parallel and perpendicular to the fibers $[6,10,11]$. When multiple lamina are assembled into a laminate, a global principle material coordinate system is established. Stacking sequence refers to the order in which lamina are assembled and determine which special types of laminates they may be. Simple laminates would be labeled as [0/45/90] which corresponds to one layer oriented at 0 degrees, the next layer at 45 degrees, and the third layer at 90 degree. If a layer in a specific orientation was repeated, then an example would be $\left[0_{2}, 90_{3}\right]$. If the entire stacking sequence was repeated multiple times then a subscript would fall outside of the bracket, $[0,90]_{3}$. If a laminate was symmetric about the middle surface then an example notation would be $[0,45,90]_{s}$ where the subscript $s$ is used. Categories of laminates based on stacking sequence follows $[6,10]$ :

Balanced Laminate: Each $+\theta$ ply is balanced with a $-\theta$ ply but does not include $0^{\circ}$ and $90^{\circ}$ plies.

Symmetric Laminate: Laminate plies are mirrored about the geometric midplane.

Angle Ply Laminate: Laminate has plies oriented at angles other than the principle material directions.

Some other important terms are [10]:

Homogeneous: Material properties are the same throughout the material at any point. Composites are generally considered heterogeneous (non-homogeneous) due to the combination of matrix and fiber. However, when discussing the linear elastic behavior of laminates, the material may be generalized as homogeneous (referred to as smearing).

Anisotropic: Material properties are not the same in any direction.

Isotropic: Material properties are the same in all directions. Laminates are not isotropic.

Transversely Isotropic: There exists one plane that has material properties that are the same in any direction of said plane.

Orthotropic: Different material properties exist in three perpendicular planes. These properties are direction specific. 
Laminated composites are usually made of continuous fiber-reinforcement with the assumption that the material in question is made up of one or more plies (layers) with each ply consisting of fibers uniformly parallel and continuous, the material can be examined in a plane stress scenario, and that the thickness is much smaller than the length or width $[6,10]$.

\subsubsection{Classical Lamination Theory}

When studying the stress-strain behavior of fiber-reinforced laminates, classical lamination theory (CLT) is typically used. The elastic constants for this theory are derived from a study of the macromechanics of a lamina, the micromechanics of a lamina, and the macromechanics of a laminate.

\subsubsection{Macromechanics of a Lamina}

Generally, Hooke's Law for anisotropic materials can be described by the equation:

$$
\sigma_{i}=C_{i j} \epsilon_{j}
$$

where $C_{i j}$ is the stiffness tensor characterized by 36 material constants.

$$
\left[\begin{array}{c}
\sigma_{11} \\
\sigma_{22} \\
\sigma_{33} \\
\sigma_{44} \\
\sigma_{55} \\
\sigma_{66}
\end{array}\right]=\left[\begin{array}{llllll}
C_{11} & C_{12} & C_{13} & C_{14} & C_{15} & C_{16} \\
C_{21} & C_{22} & C_{23} & C_{24} & C_{25} & C_{26} \\
C_{31} & C_{32} & C_{33} & C_{34} & C_{35} & C_{36} \\
C_{41} & C_{42} & C_{43} & C_{44} & C_{45} & C_{46} \\
C_{51} & C_{52} & C_{53} & C_{54} & C_{55} & C_{56} \\
C_{61} & C_{62} & C_{63} & C_{64} & C_{65} & C_{66}
\end{array}\right]\left[\begin{array}{c}
\epsilon_{11} \\
\epsilon_{22} \\
\epsilon_{33} \\
\epsilon_{44} \\
\epsilon_{55} \\
\epsilon_{66}
\end{array}\right]
$$

When an orthogonal material is being studied, the stiffness tensor can be simplified to contain nine material constants 


$$
\left[\begin{array}{c}
\sigma_{11} \\
\sigma_{22} \\
\sigma_{33} \\
\sigma_{44} \\
\sigma_{55} \\
\sigma_{66}
\end{array}\right]=\left[\begin{array}{cccccc}
C_{11} & C_{12} & C_{13} & 0 & 0 & 0 \\
C_{21} & C_{22} & C_{23} & 0 & 0 & 0 \\
C_{31} & C_{32} & C_{33} & 0 & 0 & 0 \\
0 & 0 & 0 & C_{44} & 0 & 0 \\
0 & 0 & 0 & 0 & C_{55} & 0 \\
0 & 0 & 0 & 0 & 0 & C_{66}
\end{array}\right]\left[\begin{array}{c}
\epsilon_{11} \\
\epsilon_{22} \\
\epsilon_{33} \\
\epsilon_{44} \\
\epsilon_{55} \\
\epsilon_{66}
\end{array}\right]
$$

For transversely isotropic materials, Hooke's Law simplifies further to only five material constants. For example, the following equation shows isotropy in the 2-3 plane:

$$
\left[\begin{array}{c}
\sigma_{11} \\
\sigma_{22} \\
\sigma_{33} \\
\sigma_{44} \\
\sigma_{55} \\
\sigma_{66}
\end{array}\right]=\left[\begin{array}{cccccc}
C_{11} & C_{12} & C_{13} & 0 & 0 & 0 \\
C_{21} & C_{22} & C_{23} & 0 & 0 & 0 \\
C_{31} & C_{32} & C_{33} & 0 & 0 & 0 \\
0 & 0 & 0 & \frac{C_{22}-C_{23}}{2} & 0 & 0 \\
0 & 0 & 0 & 0 & C_{55} & 0 \\
0 & 0 & 0 & 0 & 0 & C_{55}
\end{array}\right]\left[\begin{array}{c}
\epsilon_{11} \\
\epsilon_{22} \\
\epsilon_{33} \\
\epsilon_{44} \\
\epsilon_{55} \\
\epsilon_{66}
\end{array}\right]
$$

And for an isotropic material:

$$
\left[\begin{array}{c}
\sigma_{11} \\
\sigma_{22} \\
\sigma_{33} \\
\sigma_{44} \\
\sigma_{55} \\
\sigma_{66}
\end{array}\right]=\left[\begin{array}{cccccc}
C_{11} & C_{12} & C_{13} & 0 & 0 & 0 \\
C_{21} & C_{22} & C_{23} & 0 & 0 & 0 \\
C_{31} & C_{32} & C_{33} & 0 & 0 & 0 \\
0 & 0 & 0 & \frac{C_{11}-C_{12}}{2} & 0 & 0 \\
0 & 0 & 0 & 0 & \frac{C_{11}-C_{12}}{2} & 0 \\
0 & 0 & 0 & 0 & 0 & \frac{C_{11}-C_{12}}{2}
\end{array}\right]\left[\begin{array}{c}
\epsilon_{11} \\
\epsilon_{22} \\
\epsilon_{33} \\
\epsilon_{44} \\
\epsilon_{55} \\
\epsilon_{66}
\end{array}\right]
$$

For plane stress considerations, when the lamina is considered sufficiently thin, and stresses through the thickness are considered negligible, the plane stress Hooke's Law for an orthotropic lamina is given as: 


$$
\left[\begin{array}{c}
\sigma_{1} \\
\sigma_{2} \\
\tau_{x y}
\end{array}\right]=\left[\begin{array}{ccc}
Q_{11} & Q_{12} & 0 \\
Q_{21} & Q_{22} & 0 \\
0 & 0 & Q_{66}
\end{array}\right]\left[\begin{array}{c}
\epsilon_{1} \\
\epsilon_{2} \\
\gamma_{12}
\end{array}\right]
$$

Rearranged for strain-stress:

$$
\left[\begin{array}{c}
\epsilon_{1} \\
\epsilon_{2} \\
\gamma_{12}
\end{array}\right]=\left[\begin{array}{ccc}
S_{11} & S_{12} & 0 \\
S_{21} & S_{22} & 0 \\
0 & 0 & S_{66}
\end{array}\right]\left[\begin{array}{c}
\sigma_{1} \\
\sigma_{2} \\
\tau_{x y}
\end{array}\right]
$$

where $Q_{i j}$ and $S_{i j}$ are the reduced stiffness coefficients and reduced compliance coefficients, respectively $[6,11]$.

$$
\begin{aligned}
Q_{11} & =\frac{E_{1}}{1-\nu_{21} \nu_{12}} \\
Q_{12} & =\frac{\nu_{12} E_{2}}{1-\nu_{21} \nu_{12}} \\
Q_{22} & =\frac{E_{2}}{1-\nu_{21} \nu_{12}} \\
Q_{66} & =G_{12}
\end{aligned}
$$

The reduced stiffness and reduced compliance matrices are related by:

$$
[Q]=[S]^{-1}
$$

The final consideration is when the fibers are not orientated in a principle material direction. Fibers on the lamina local coordinate system $(1,2,3)$ thus are transformed to the global coordinate system $(\mathrm{x}, \mathrm{y}, \mathrm{z})$ based on angle $\theta$ through the use of the transformation matrix, $\mathrm{T}$ : 


$$
[T]=\left[\begin{array}{ccc}
\cos ^{2}(\theta) & \sin ^{2}(\theta) & 2 \cos (\theta) \sin (\theta) \\
\sin ^{2}(\theta) & \cos ^{2}(\theta) & -2 \cos (\theta) \sin (\theta) \\
-\cos (\theta) \sin (\theta) & \cos (\theta) \sin (\theta) & \cos ^{2}(\theta)-\sin ^{2}(\theta)
\end{array}\right]
$$

The application of the transformation matrix to lamina stresses would be as follows:

$$
\left[\begin{array}{c}
\sigma_{1} \\
\sigma_{2} \\
\tau_{12}
\end{array}\right]=[T]\left[\begin{array}{c}
\sigma_{x} \\
\sigma_{y} \\
\tau_{x y}
\end{array}\right]
$$

Conversely,

$$
\left[\begin{array}{c}
\sigma_{x} \\
\sigma_{y} \\
\tau_{x y}
\end{array}\right]=[T]^{-1}\left[\begin{array}{c}
\sigma_{1} \\
\sigma_{2} \\
\tau_{12}
\end{array}\right]
$$

The equation to obtain $\bar{Q}$ for angle $\theta$ is

$$
\bar{Q}=T^{-1} Q T
$$

When transforming strains from global to local coordinates the rotation matrix $[R]$ is used in the manner:

$$
[R]=\left[\begin{array}{lll}
1 & 0 & 0 \\
0 & 1 & 0 \\
0 & 0 & 2
\end{array}\right]
$$

Transforming strains is done with the formulas:

$$
\left[\begin{array}{c}
\epsilon_{1} \\
\epsilon_{2} \\
\tau_{12}
\end{array}\right]=[R][T][R]^{-1}\left[\begin{array}{c}
\epsilon_{x} \\
\epsilon_{y} \\
\tau_{x x y}
\end{array}\right]
$$


Hence, the relationship between global stress and global strain can be written:

$$
\left[\begin{array}{c}
\sigma_{x} \\
\sigma_{y} \\
\tau_{x y}
\end{array}\right]=[T]^{-1}[Q][R][T][R]^{-1}\left[\begin{array}{c}
\epsilon_{x} \\
\epsilon_{y} \\
\tau_{x x y}
\end{array}\right]
$$

Note that

$$
\bar{Q}=[T]^{-1}[Q][R][T][R]^{-1}
$$

Thus, the global stress-strain relation becomes:

$$
[\sigma]=[\bar{Q}][\epsilon]
$$

\subsubsection{Micromechanics of a Lamina}

Micromechanics of a lamina involve using the individual material properties and volume fractions to determine the engineering constants such as the elastic modulus, Poisson's ratio, and the shear modulus. Smearing assumptions are applied such that the stress-strain behavior is considered linear homogeneous, and isotropic. Fibers are assumed to be linear elastic, distributed evenly in the lamina, and there is an absence of imperfections on the fiber/matrix interface.

The individual material properties of interest are the modulus of elasticity for the fiber $\left(E_{f}\right)$ and matrix $\left(E_{m}\right)$, Poisson's ratio for the fiber $\left(\nu_{f}\right)$ and matrix $\left(\nu_{m}\right)$, the shear moduli for the fiber $\left(G_{f}\right)$ and matrix $\left(G_{m}\right)$, and thermal expansion coefficients for fiber $\left(\alpha_{f}\right)$ and matrix $\left(\alpha_{m}\right)$. The engineering constants for the lamina can then be calculated from the following formulas $[6,10,12]$ :

$$
\begin{aligned}
E_{1} & =E_{f} V_{f}+E_{m} V_{m} \\
E_{2} & =\frac{E_{f} E_{m}}{E_{f} V_{m}+E_{m} V_{f}} \\
\nu_{12} & =\nu_{f} V_{f}+\nu_{m} V_{m} \\
G_{12} & =\frac{G_{f} G_{m}}{G_{f} V_{m}+G_{m} V_{f}} \\
\alpha_{1} & =\frac{E_{f} \alpha_{f} V_{f}+E_{m} \alpha_{m} V_{m}}{E_{f} V_{f}+E_{m} V_{m}} \\
\alpha_{2} & =\left(1+V_{f}\right) \alpha_{f} V_{f}+\left(1+V_{m}\right) \alpha_{m} V_{m}-\alpha_{1}\left(V_{f}^{2}+V_{m}^{2}\right)
\end{aligned}
$$




\subsubsection{Macromechanics of a Laminate}

Now that the foundation and stress-strain relation has been demonstrated for a lamina, classical lamination theory (CLT) can be defined. For CLT to hold true, the following assumptions are made $[6,11]$ :

1. The displacements are continuous throughout the laminate domain.

2. The Kirchoff hypothesis is assumed to be valid and the midplanes can be used to show the shape of the laminate.

3. The strain-displacement relation is linear.

4. Linear-elastic behavior.

5. The out-of-plane through thickness stresses are sufficiently small, therefore plane stress.

6. Ideal bonding of lamina.

The Kirchoff hypothesis is that the midplane surface for a three dimensional plate can be used to represent a two dimensional model. Additionally, the hypothesis says that (1) straight lines remain straight before and after deformation, (2) transverse normals are inextensible, and (3) the transverse normal rotate to remain perpendicular the midsurface during deformation. Based on the Kirchoff assumptions, any point in $(x, y, z)$ is displaced by a vector $u=u e_{x}+v e_{y}+z e_{z}$. Applying the Green strain tensor and the infinitesimal strain tensor, and the second Piola-Kirchoff stress tensor and Cauchy stress tensor, it is required that the displacements be defined as [11]:

$$
\begin{aligned}
u(x, y, z, t) & =u_{0}(x, y, t)-z \frac{\partial w_{0}}{\partial x} \\
v(x, y, z, t) & =v_{0}(x, y, t)-z \frac{\partial w_{0}}{\partial y} \\
w(x, y, z, t) & =w_{0}(x, y, t)
\end{aligned}
$$

Where the displacements $u_{0}, v_{0}$, and $w_{0}$ are that of the midplane. The associated strains can be calculated either using the linear strain-displacement relation or the nonlinear strain-displacement relation. The nonlinear strains are shown [11]:

$$
E_{x x}=\frac{\partial u}{\partial x}+\frac{1}{2}\left[\left(\frac{\partial u}{\partial x}\right)^{2}+\left(\frac{\partial v}{\partial x}\right)^{2}+\left(\frac{\partial w}{\partial x}\right)^{2}\right]
$$




$$
\begin{aligned}
& E_{y y}=\frac{\partial v}{\partial y}+\frac{1}{2}\left[\left(\frac{\partial u}{\partial y}\right)^{2}+\left(\frac{\partial v}{\partial y}\right)^{2}+\left(\frac{\partial w}{\partial y}\right)^{2}\right] \\
& E_{z z}=\frac{\partial w}{\partial z}+\frac{1}{2}\left[\left(\frac{\partial u}{\partial z}\right)^{2}+\left(\frac{\partial v}{\partial z}\right)^{2}+\left(\frac{\partial w}{\partial z}\right)^{2}\right] \\
& E_{x y}=\frac{1}{2}\left(\frac{\partial u}{\partial y}+\frac{\partial v}{\partial x}+\frac{\partial u \partial u}{\partial x \partial y}+\frac{\partial v \partial v}{\partial x \partial y}+\frac{\partial w \partial w}{\partial x \partial y}\right) \\
& E_{x z}=\frac{1}{2}\left(\frac{\partial u}{\partial z}+\frac{\partial w}{\partial x}+\frac{\partial u \partial u}{\partial x \partial z}+\frac{\partial v \partial v}{\partial x \partial z}+\frac{\partial w \partial w}{\partial x \partial z}\right) \\
& E_{y z}=\frac{1}{2}\left(\frac{\partial v}{\partial z}+\frac{\partial w}{\partial y}+\frac{\partial u \partial u}{\partial y \partial z}+\frac{\partial v \partial v}{\partial y \partial z}+\frac{\partial w \partial w}{\partial y \partial z}\right)
\end{aligned}
$$

If the small strain assumption was applied then all 2nd order strain terms can be considered negligible so long as the displacement gradients are linear. Additionally, if the transverse normal rotations $\partial w_{0} / \partial x$ and $\partial w_{o} / \partial y$ are moderate (10-15 degrees), then these three terms cannot be considered negligible compared to $\epsilon$ :

$$
\left(\frac{\partial w}{\partial x}\right)^{2},\left(\frac{\partial w}{\partial y}\right)^{2},\left(\frac{\partial w}{\partial x} \frac{\partial w}{\partial y}\right)
$$

Thus, considering the above assumptions and displacement field, the associated strains for small strains and moderate rotations:

$$
\begin{aligned}
\epsilon_{x x} & =\frac{\partial u}{\partial x}+\frac{1}{2}\left(\frac{\partial w}{\partial x}\right)^{2}-z \frac{\partial^{2} w_{o}}{\partial x^{2}} \\
\epsilon_{x y} & =\frac{1}{2}\left(\frac{\partial u}{\partial y}+\frac{\partial v}{\partial x}+\frac{\partial w}{\partial x} \frac{\partial w}{\partial y}\right)-z \frac{\partial^{2} w_{0}}{\partial x \partial y} \\
\epsilon_{y y} & =\frac{\partial v}{\partial y}+\frac{1}{2}\left(\frac{\partial w}{\partial y}\right)^{2}-z \frac{\partial^{2} w_{0}}{\partial y^{2}} \\
\epsilon_{x z} & =\frac{1}{2}\left(\frac{\partial u}{\partial z}+\frac{\partial w}{\partial x}\right) \\
\epsilon_{z z} & =\frac{\partial w}{\partial z} \\
\epsilon_{y z} & =\frac{1}{2}\left(\frac{\partial v}{\partial z}+\frac{\partial w}{\partial y}\right)
\end{aligned}
$$

These strains are called the von Karmin strains of von Karmin plate theory. Here, considering thin plate theory assumptions, the transverse strains $\left(\epsilon_{x z}, \epsilon_{y z}, \epsilon_{z z}\right)$ can be equal to zero. The strains can further be written in their midplane forms [11]: 


$$
\epsilon^{0}=\left[\begin{array}{c}
\epsilon_{x}^{0} \\
\epsilon_{y}^{0} \\
\epsilon_{x} y^{0}
\end{array}\right]=\left[\begin{array}{c}
\frac{\partial u^{0}}{\partial x}+\frac{1}{2}\left(\frac{\partial w^{0}}{\partial x}\right)^{2} \\
\frac{\partial v^{0}}{\partial y}+\frac{1}{2}\left(\frac{\partial w^{0}}{\partial y}\right)^{2} \\
\frac{1}{2}\left(\frac{\partial u^{0}}{\partial y}+\frac{\partial v^{0}}{\partial x}+\frac{\partial w^{0}}{\partial x}\left(\frac{\partial w^{0}}{\partial y}\right)\right.
\end{array}\right]
$$

Equation 2.44 are the membrane strains and Equations 2.45-2.47are the bending strains (curvatures) [11]. From these equations, the strain for any point in the domain can be calculated using the total strain relation:

$$
\left[\begin{array}{c}
\epsilon_{x} \\
\epsilon_{y} \\
\epsilon_{x y}
\end{array}\right]=\left[\begin{array}{c}
\epsilon_{x}^{0} \\
\epsilon_{y}^{0} \\
\epsilon_{x y}^{0}
\end{array}\right]+z\left[\begin{array}{c}
\kappa_{x} \\
\kappa_{y} \\
\kappa_{x y}
\end{array}\right]
$$

The importance of the inclusion of von Karmin strains is that it has been found that although CLT smearing can accurately capture static deflections, natural vibration frequencies and mode shapes, buckling loads and mode shapes, and thermal expansion coefficients, it cannot be relied upon to predict the response of thicker laminates, predict edge response behavior, or predict room temperature shapes of thin unsymmetric laminates [4].

\subsubsection{Constitutive Relations for CLT}

Practically, thin and moderately thick laminates, which have a thickness that is small compared to the in-plane dimensions, is in a state of plane stress [11]. Thus, the plane stress reduced constitutive equations are used. Thus, using Hookes Law, the stresses for each $k^{t h}$ ply are given by $[6,10,11]$ : 


$$
[\sigma]_{k}=[\bar{Q}]_{k}[\epsilon]_{k}
$$

and expanding this equation to include midplane strains as defined in Eq. 2.48,

$$
\left[\begin{array}{c}
\sigma_{x} \\
\sigma_{y} \\
\sigma_{x y}
\end{array}\right]_{k}=\left[\begin{array}{lll}
\bar{Q}_{11} & \bar{Q}_{12} & \bar{Q}_{16} \\
\bar{Q}_{21} & \bar{Q}_{22} & \bar{Q}_{26} \\
\bar{Q}_{16} & \bar{Q}_{26} & \bar{Q}_{66}
\end{array}\right]_{k}\left[\left[\begin{array}{c}
\epsilon_{x}^{0} \\
\epsilon_{y}^{0} \\
\gamma_{x y}^{0}
\end{array}\right]+z\left[\begin{array}{c}
\kappa_{x} \\
\kappa_{y} \\
\kappa_{x y}
\end{array}\right]\right]
$$

Eq. 2.50 allows the stress-strain relationship for a laminate to be identified. It is important to note that $[\bar{Q}]$ may not be consistent through the thickness of the laminate due to possible variations in lamina orientations. This means that strains vary continuously through the thickness while due to ply interactions stress does not. Therefore, it is considerably advantageous to define the forces and moments in terms of the stresses. This is done through the force and moment resultant integrations:

$$
\begin{aligned}
N_{x} & =\int_{-t / 2}^{t / 2} \sigma_{x} d z \\
M_{x} & =\int_{-t / 2}^{t / 2} \sigma_{x} z d z
\end{aligned}
$$

Integrating Eq. 2.51-2.52 yield over the laminate thickness, $t$, yields the following two equations:

$$
\begin{aligned}
& {\left[\begin{array}{c}
N_{x} \\
N_{y} \\
N_{x y}
\end{array}\right]=\left[\begin{array}{lll}
A_{11} & A_{12} & A_{16} \\
A_{21} & A_{22} & A_{26} \\
A_{16} & A_{26} & A_{66}
\end{array}\right]\left[\begin{array}{c}
\epsilon_{x}^{0} \\
\epsilon_{y}^{0} \\
\gamma_{x y}^{0}
\end{array}\right]+\left[\begin{array}{lll}
B_{11} & B_{12} & B_{16} \\
B_{21} & B_{22} & B_{26} \\
B_{16} & B_{26} & B_{66}
\end{array}\right]\left[\begin{array}{c}
\kappa_{x} \\
\kappa_{y} \\
\kappa_{x y}
\end{array}\right]} \\
& {\left[\begin{array}{l}
M_{x} \\
M_{y} \\
M_{x y}
\end{array}\right]=\left[\begin{array}{lll}
B_{11} & B_{12} & B_{16} \\
B_{21} & B_{22} & B_{26} \\
B_{16} & B_{26} & B_{66}
\end{array}\right]\left[\begin{array}{c}
\epsilon_{x}^{0} \\
\epsilon_{y}^{0} \\
\gamma_{x y}^{0}
\end{array}\right]+\left[\begin{array}{lll}
D_{11} & D_{12} & D_{16} \\
D_{21} & D_{22} & D_{26} \\
D_{16} & D_{26} & D_{66}
\end{array}\right]\left[\begin{array}{c}
\kappa_{x} \\
\kappa_{y} \\
\kappa_{x y}
\end{array}\right]}
\end{aligned}
$$

Where the $A_{i j}$ are the extensional stiffnesses, $B_{i j}$ are the bending-extensional stiffnesses, and the $D_{i j}$ are the bending stiffness. These can all be defined in terms of the lamina stiffness $\left(\bar{Q}_{i j k}\right)$ as functions of stacking positions in the laminate: 


$$
\begin{aligned}
A_{i j} & =\sum_{k=1}^{N} Q_{i j}^{k}\left(z_{k}-z_{k-1}\right) \\
B_{i j} & =\frac{1}{2} \sum_{k=1}^{N} Q_{i j}^{k}\left(z_{k}^{2}-z_{k-1}^{2}\right) \\
D_{i j} & =\frac{1}{3} \sum_{k=1}^{N} Q_{i j}^{k}\left(z_{k}^{3}-z_{k-1}^{3}\right)
\end{aligned}
$$

$[\mathrm{A}],[\mathrm{B}],[\mathrm{D}]$ are $3 \mathrm{x} 3$ symmetric laminate coefficient matrices that are related to the stacking se-

quences. When considering thermal effects, the thermal resultant forces and moments are $[11,6,10]$ :

$$
\begin{aligned}
N_{i}^{t h} & \left.==\sum_{k=1}^{N} \sum_{k=1}^{N} Q_{i j}^{k} \alpha_{j}^{k}\right)\left(z_{k}-z_{k-1}\right) \Delta T \\
M_{i}^{t h} & \left.==\frac{1}{2} \sum_{k=1}^{N} \sum_{k=1}^{N} Q_{i j}^{k} \alpha_{j}^{k}\right)\left(z_{k}^{2}-z_{k-1}^{2}\right) \Delta T
\end{aligned}
$$

Combining all of this, the contracted laminate constitutive force-strain relations are:

$$
\left[\begin{array}{l}
N \\
M
\end{array}\right]=\left[\begin{array}{ll}
A & B \\
C & D
\end{array}\right]\left[\begin{array}{l}
\epsilon^{0} \\
\kappa
\end{array}\right]
$$

This concludes the overview of applicable extended classical lamination theory which included the von Karmin strains. Typically, it was found that CLT worked well for most laminates except in specific instances, one of those being the prediction of stable equilibrium shapes for thin asymmetric laminates.

\subsection{Bistable Laminates Cure Shape Prediction}

Bistable laminates research history can be divided into predictions of room temperature cure shapes, inducing or actuating snapthrough behavior, and application use. 


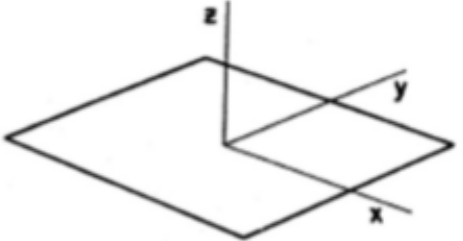

(a)

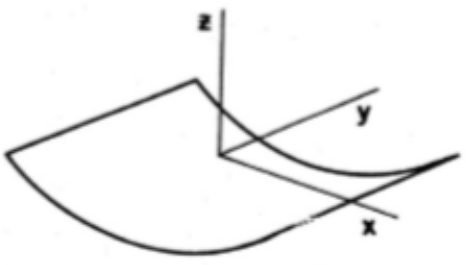

(c)

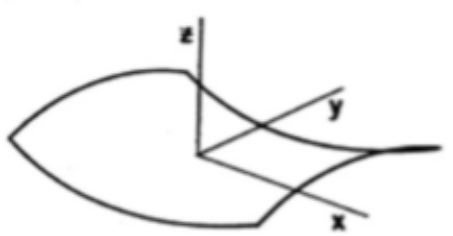

(b)

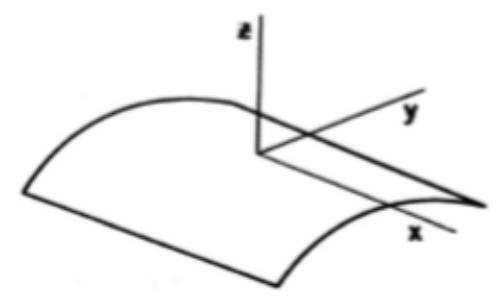

(d)

Figure 2.1: Possible Laminate Shapes (a) Flat (b) Unstable Saddle Shape $\kappa_{x}=\kappa_{y}$ (c) and (d) Stable Cylindrical Shapes of Equal Opposite Perpendicular Axis Curvatures (With permission to republish $[4])$.

\subsubsection{Analytical Development}

Asymmetric bistable composites were first widely studied by Hyer in his 1981 paper. In this paper, he was able to pique much interest by stating possible applications in shape-morphing and actuation materials [4]. His work experimentally investigated bistable phenomenon behavior and how it was not predictable through classical lamination theory unless von Karmin plate theory was included. The von Karmin plate theory was adequate in extending viable descriptions of the geometric nonlinearities that CLT did not capture due to disinclusion of moderate rotations. Furthermore, it was stated that bistable behaviors source was due to the residual stresses created from mismatched principle thermal expansion coefficients which originally caused disinterest due to coupled bending-extension behavior $[4,13]$. However, Hyer was able to show accurate predictions of the post-cure shape cylindrical curvatures could be made using von Karmin plate theory, that thicker laminates saddle shapes could be predicted by CLT, and that snapthrough shapes at room temperatures had curvatures which were inversely related through equal and opposite magnitudes $[4,13,14]$.

Hyer developed an analytical model that included the effects of thermal expansion to predict 

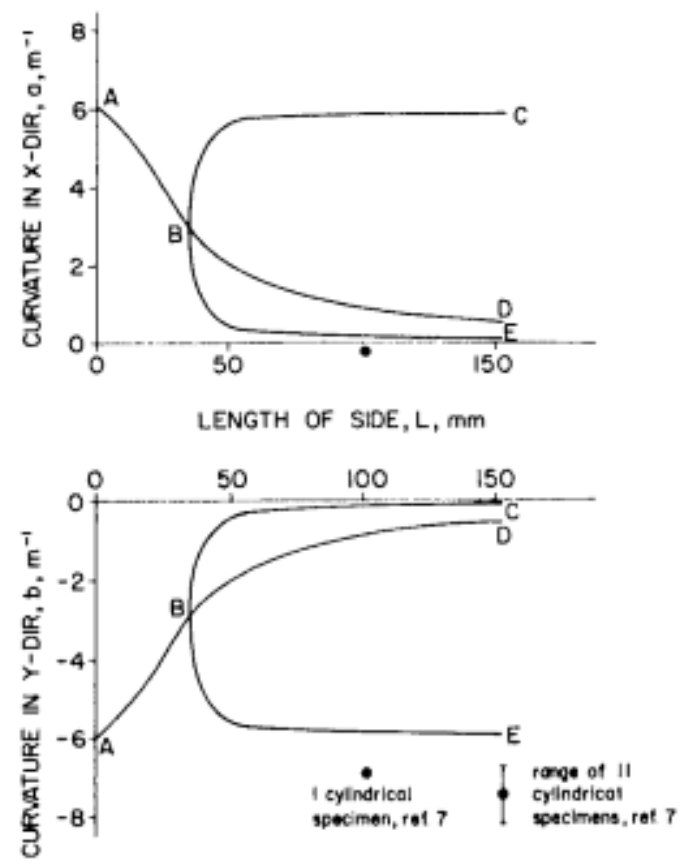

Figure 2.2: Hyer's Bifurcation Diagram $\left[0_{2} / 90_{2}\right]_{T}$ and $\left[0_{4} / 90_{4}\right]_{T} 150 \mathrm{~mm} x 150 \mathrm{~mm}$ T300/5208 laminates. Path ABD is predicted by CLT as saddle shape. Branches ABC and ABE are two stable cylindrical shapes. Point B is the critical length bifurcation (With permission to republish[4]).

the post cure shapes using the Rayleigh-Ritz minimization of potential energy $[4,13]$. This model allowed Hyer to create bifurcation diagrams which showed the curvature of four-ply graphite-epoxy laminates. These bifurcation diagrams showed a critical sidelength that corresponded to a bifurcation point.

This critical point indicated that laminates whose sidelength were less than the critical length could be described accurately through classical lamination theory. Conversely, laminates that had sidelengths greater than this critical length exhibited bistable behavior via the indication of two possible curvatures. Hyer also suggested that the laminates were susceptible to the effects of moisture absorption, viscoelastic relaxation, and any other mechanism that could have an effect on internal stresses would affect the critical length $[2,4,6]$. Hyer, lastly suggested, that asymmetric curing, cooling, and moisture absorption could result in one of the two bistable configurations having an increased snapthrough actuation force in one direction compared to snapback.

Hyer continued his work by including $[0 / 0 / 0 / 90]$ and $[0 / 0 / 90 / 0]$ layups realizing that the 
out-of-plane deflections were a multiple order of magnitude of the thickness, he concluded that the strain-displacement relationship was nonlinear, and thus included additional terms in his analytical model $[14,15]$. Additionally, he found that if a laminate was just above the critical length threshold that the effects of moisture ingress could cause a laminate to fall just below the critical length threshold and snapthrough would occur without actuation loads. However, since the results were only concerned with room-temperature shapes, did not include shape-temperature relationships, and all laminates were assumed to be orthotropic; thus not accounting for imperfections, his updated theory did not show significant changes in the out-of-plane displacements or inplane strains [14].

The shape-temperature relationship was unable to be studied due to the autoclaving or vacuum bag fabrication processes. These processes constrained the laminates to the molds and were invisible due to the use of the vacuum bags which rendered deformation observations and measurements impossible [14]. It was assumed that the cooling process was reversible so Hamamoto and Hyer reheated laminates, removed them from heat, and measured deformation during the subsequent cooling. Including geometric variances in fabrication (lamina orientation, thickness, and heat distribution), they found another source of imperfections which was that with just as as little as $1 \%$ variation in lamina thickness was influential enough on the curvature-temperature curves to make the bifurcation point disappear $[14,16,17]$. This is significant since, it is expected that there be $\pm 2 \%$ thickness variance in lamina due to manufacturing $[14,18]$. Additionally, Hamamoto and Hyer found that when they added additional terms to the displacement polynomial approximation to account for inplane shear strain, they found that shear strain changes were negligible for square laminates with small or large length-to-thickness aspect ratios. For shear strains between large and small aspect ratios, the effects were found ot be significant [19].

Hyer's theory was then applied to angle-ply laminates which showed an exhibition of a new twist curvature term in the out-of-plane displacement polynomial $[14,20]$. New approximation polynomials were used but resulted in non-ideal solution cases. Both Emam and Tawfik state that several back and forth iterative studies were done with no significant improvements, including an angle-ply study that transformed structural coordinate system curvatures to principle coordinate curvatures $[14,19,20,21,22,23,24]$. Notably, Mattioni et. al refines curvature prediction by no longer assuming a constant curvature across the domain by introducing a fourth order polynomial for out-of-plane displacement which also maintained in-plane strain assumptions [5, 14]. Mattioni's work modified Hyer's displacement function in an attempt to also try and capture edge behavior 
more accurately. Up to this point, Hyer had been using the out-of-plane displacement [5]:

$$
w(x, y)=-\frac{1}{2}\left(w_{20} x^{2}+w_{02} y^{2}+w_{11} x y\right)
$$

where $w_{20}$ and $w_{02}$ are the constant curvature for $x$ and $y$, respectively, while $w_{11}$ is the twist curvature. The fourth order polynomial was introduced to the displacement function as the product of two parabolas [5]:

$$
\begin{aligned}
w(x, y) & =P(x) Q(y) \\
P(x) & =p_{0}+p_{1} x+p_{2} x^{2} \\
Q(y) & =g_{o}+g_{1} y+g_{2} y^{2}
\end{aligned}
$$

giving the displacement function as:

$$
w(x, y)=w_{00}+w_{10} x+w_{01} y+w_{20} x^{2}+w_{02} y^{2}+w_{11} x y+w_{12} x y^{2}+w_{21} x^{2} y+w_{22} x^{2} y^{2}
$$

Not changing any other assumptions of Hyer's theory, Mattioni created a system with 20 unknown parameters. However, the results of Mattioni's model for $\left[0_{4}, 90_{4}\right] 180 \mathrm{~mm}$ square CFRP plates predicted parabolic edges (whereas Hyer's model produces flat edge) providing marginal improvement. Using this model, Mattioni's method worked for non-free edge boundaries during his piecewise layup studies where one half of the laminate was flat and the other half was bistable. Thus, Mattioni was able to study the effects of non-free edge boundary conditions.

A. Pierrera coupled the analytical approach with a path-following algorithm increasing the out-of-plane displacement polynomial approximation to the $11^{\text {th }}$ order. However, even with more favorable results, finite element analysis (FEA) was found to perform better for edge displacement descriptions $[9,14,21,25]$.

\subsubsection{Finite Element Analysis Cure Shape Development}

Finite Element Methods were used to model unsymmetric multistable laminates due to the ease of visualization and measuring cure shapes with acceptable accuracy. Emam states that 


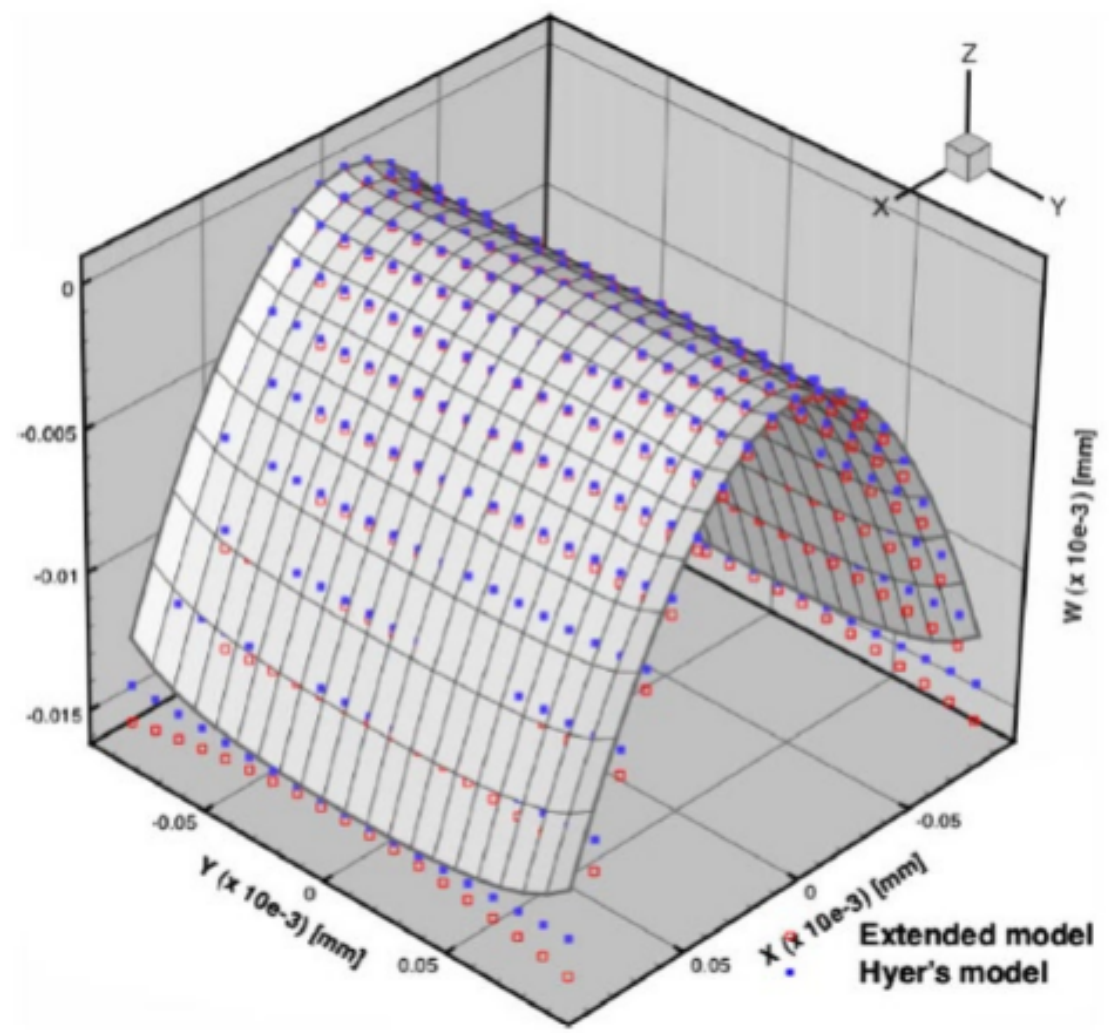

Figure 2.3: Mattioni's Out-of-Plane Displacement Predictions vs. Hyer's 1996 Model. Note the Straight Edge Predicted by Hyer and the Parabolic Edge by Mattioni. Permission to republish from $[5]$ 
the one downside of utilizing the FEM was that finding other equilibirum solutions was not easy or straightforward [14]. Betts et al. found that arbitrary layup laminate shapes were able to be captured adequately through FEM but the results were sensitive to imperfecctions resulted from temperature variations, ply thickness, edge conditions, and matrix variation [14, 18]. Moreover, Giddings et al. found with ANSYS software which included imperfection details was able to better account for cure shape predictions $[14,26]$. Furthermore, Brampton et al. found that laminate shape sensitivity was most directly affected by thermal expansion coefficients, moisture absorption, and individual ply parameters $[14,17]$. Tawfik found the same sensitivity issues were apparent when he studied unsymmetric laminates with Abaqus FEA software exploring the effects of varying geometries and environmental effects and established detailed procedures [21]. Kemmann and Myers investigated the effect of partially symmetric piecewise laminates (similar to Mattioni's piecewise studies) and how boundary conditions influenced bistability in Abaqus finding that up to $83 \%$ symmetry can be introduced until bistability is lost [2]. Phatak examined the effects of varying length, width, and thickness of rectangular laminates. Ultimately, using Abaqus, Phatak determined that there existed a geometric critical ratio for sidelength-to-ply of 1.2:1 (in inches-to-number of plies) describing the critical loss of bistability [9]. This key ratio signifies a distinction in comparison to Rayleigh-Ritz models. All of these studies were compared with experimental results which provided some level of validation.

\subsection{Bistable Laminates Static Snapthrough Behavior}

Because bistable laminates exhibit an inherent ability to shape-morph between two equilibrium positions, studies have been done to characterize this behavior. The official nomenclature used to describe this shape-morphing phenomena is "snapthrough." Snapthrough occurs when sufficient external forces or moments have been applied to a bistable laminate. Snapthrough usually occurs rapidly once sufficient energy has been introduced to the system. Additionally, the laminate will not require any external loads to hold the structure in the new position. Identifying these snapthrough forces and loads is paramount to bistable behavior description [3, 14].

Dano and Hyer first studied snapthrough behavior of unsymmetric cross-ply laminates with external applied edge forces that resultant in moments. Dano and Hyer modeled the behavior using a Rayleigh-Ritz approach coupled with virtual work principles which gave favorable correlation 
[27]. Schlecht and Schulte continued snapthrough studies by examining edge actuation while keeping the center fixed with FEA software [28]. Dano and Hyer continued their work by examining three different families of bistable unsymmetric laminates by way of the Rayleigh-Ritz and virtual work approximations [24]. Futhermore, they were able to predict forces and moments with decent accuracy and suggested the use of a smart material to cause actuation [14, 24]. Emam states that the work by Dano and Hyer was approximate, did not account for polymer relaxation during snapthrough and that snapthrough load prediction was on average $40 \%$ higher $[14,24,29]$. Diaconu et al. attributes the high predictions for snapthrough or "buckling" to analytical model restrictions following shapes with constant curvature [29]. Meanwhile Pirrera suggested that higher-order displacement polynomials would alleviate the analytical buckling load predictions [25].

Tawfik used Abaqus and experimental comparisons to model both the cure shape and static snapthrough behavior while considering various sidelength-to-thickness ratios [14, 21]. The use of an Instron Microtester applied to the center point of experimental samples allowed accurate measurements of snapthrough loads for FEA comparisons [21]. Following this, Tawfik plotted the snapthrough loads from FEA and experiments against geometric aspect ratios which showed strong correlations for his procedures [21]. Finally, Tawfik identified critical aspect ratios related to the critical bifurcation points. Cantera et al. measured both the snapthrough and snapback phenomena with each of the four corners of thin laminates fixed and an applied vertical central load using Rayleigh-Ritz extensions of Hyer's model [30]. It was found that Rayleigh-Ritz still predicted higher snapthrough and snapback loads than found in experiments and was correlated to stiffer loaddisplacements and the Hyer displacement assumption [30]. Finally, Cantera recognized that there existed a difference between snapthrough and snapback actuation loads and noted that there existed intermediate equilibrium positions during these snapping events.

\subsection{Bistable Laminates Potential Applications}

Bistable laminates have piqued a fair amount of interest due to two main characteristics exhibited. The first characteristic is their large deformation cure shapes and the second main characteristic is their ability to retain a curved surface. As such, bistable laminates could be used to create curved structures without the need for a specially crafted curved mold [21]. Another application, proposed by Schultz, was to have bistable laminates act as transformable ducts for the 
control of fluid flow [31]. Mattioni et al. proposed creating a piecewise symmetric and unsymmetric panel to be used as wing spars for a variable sweep wing, blended bistable winglets, and variable camber trailing-edges $[21,32]$. Tawfik also explored variable stacking sequences for inclusion into morphed elliptic cambered span wings [21]. Later Schultz studied airfoil structures that utilized bistable composites actuated by piezoelectrics [33]. Daynes et. al studied aeroelastic behavior of bistable helicopter blade structures created with Hexcel 913 laminates that were actuated to create trailing-edge "flapping" [34]. Moreover, several researchers have explored the use of bistable laminates combined with piezoelectrics as energy harvesters. Arrieta et al. discovered that bistable laminates bonded with piezoelectric patches were able to produce high power outputs for their size for intermittency and amplitude cycling [35]. Betts et al, found that the power output generated was high during snapthrough and optimized laminate geometry to maximize this effect concluding that square laminates were the most effective [18]. Betts et. al in another paper found that thick bistable laminates were able to increase power generation further [36]. A final potential application is the incorporation of bistable laminates to induce twist in wind turbine and/or helicopter blades to reduce torsional loads, vibrations, and increase aerodynamic efficiency [37].

\subsection{Fabrication Techniques for CFRP Structures}

Several methods are available to create CFRP structures. These methods provide a variety of options for low-cost, high-production with advanced automation to single sample creation requiring simple equipment. Another aspect for choosing a manufacturing method is the geometric complexity of the part, where a more complex design will require more effort and complexity of the fabrication process [2].

\subsubsection{Hand Layup}

The simplest method requiring the least amount of tools, hand layup involves pressing a resin-hardener epoxy matrix into a dry fibers. The resin-hardener matrix is usually measured by volume and mixed to a specific ratio. Once this is done, the matrix mixture will start setting and should be applied ot the dry fibers to saturate or wet them out. The wetted out fibers are then placed onto the mold in the desired location with a prepared peel-ply or mold release agent and allowed to dry at room-temperature for 24-72 hours or in an oven for just several hours $[2,6]$. 


\subsubsection{Vacuum Bagging}

The next simplest fabrication method is vacuum bagging where prepreg material, fiber prepared from a factory with resin mixed in, is assembled and left to cure under vacuum pressure. Prepreg material usually keep very cold to make handling easier in addition to allowing the material to be assembled without it sticking to itself. Effectively, the lower temperatures cease the curing process of the hardener in the resin. The prepreg is assembled onto a mold and covered with peel ply, breather fabric, and an air impermeable bag. Then a vacuum fitting is fitted to the bag to remove air from the closed system and pull the prepreg material together while the resin cures at room temperature or in the oven at elevated temperatures [6].

\subsubsection{Infusion}

Infusion is an advanced, somewhat automated, version of typical vacuum bagging where dry fibers are placed into a mold and resin is forced through the dry fibers until they wet out via a vacuum. The process for performing infusion is to place the fibers in a mold, use a perforated peel ply, apply a vacuum bag over top, and attach to an inlet and outlet. The inlet allows premixed resin to be drawn into the bag with a heated vacuum until the fibers are soaked through while the outlet allows any excess resin to be pulled out of the bag [6]. Usually, the system has a resin catch to inhibit resin damage to the pump and the system is closed off from the pump after enough resin has been allowed to penetrate the dry fibers.

\subsubsection{Compression Molding}

There are two types of compression molding, one method uses liquid resin pressed into dry fiber on a two part mold under high pressure. The liquid resin is injected at a high temperature use a heated hydraulic pump. A variation of this method involves used wetted out fibers that are then pressed together.

The second type is prepreg compression molding. This fabrication process is similar to that above, except prepeg fibers are placed into the two-part mold, and the mold is pressed together under heated pressure, typically in cure times requiring less than three minutes $[2,6]$. 


\section{Chapter 3}

\section{Research Design and Methodology}

This chapter describes the methods used to determine laminate shapes after the completion of the curing step, the approach used to calculate the principle deformations and curvatures, the determination of snapthrough actuation loads, energy analysis, and techniques used for experimental samples as well as study importance, delimitations, and method justification and limits.

\subsection{Purpose of the Study}

The purpose behind this study is to expand the current design space for bistable CFRP laminates. The laminates of interest are thin plate CFRP with a $\left[0_{k} / 90_{k}\right], k>0$, layup. However, the laminates have their thickness increased incrementally, where each increase in thickness risks the loss of bifurcation. In other words, increasing the thickness changes the length-to-thickness aspect ratio approaching Phatak's length-to-ply 1.2:1 determination (equivalent length-to-thickness ratio $=152$ )as well as implying increased stiffnesses and actuation loads [9]. Additional literature has not been found for laminates that push the thickness influence on bistability and have rather focused more on changing length or width dimensions or increasing the accuracy of shape predictions. In order for design engineers to make use of these laminates in the future, this research aims to describe the bistability behavior for thick bistable laminates, examining the length-to-thickness ratio, as well as providing design space information about cure shape predictions and snapthrough behavior predictions. 


\begin{tabular}{|l|c|c|}
\hline & DA409U/G35 & AS4/8552 \\
\hline \hline $\mathbf{E}_{\mathbf{1}}$ & 129 & 135 \\
$\mathbf{E}_{\text {bend }}$ & 123 & 126 \\
\hline
\end{tabular}

Table 3.1: Elastic and Flexural Moduli Comparison Table between DA409 and AS4

\begin{tabular}{|l|c|c|}
\hline & AS4/8552 & TR50S-12K/NP301 \\
\hline \hline $\mathbf{E}_{\mathbf{1}}[\mathbf{G P a}]$ & 135 & 142 \\
$\mathbf{E}_{\mathbf{2}}[\mathbf{G P a}]$ & 9.5 & 9.0 \\
$\nu_{\mathbf{1 2}}[-]$ & 0.3 & 0.29 \\
$\mathbf{G}_{\mathbf{1 2}}[\mathbf{G P a}]$ & 5 & 7.85 \\
$\mathbf{G}_{\mathbf{2 3}}[\mathbf{G P a}]$ & 3.97 & 5.23 \\
$\mathbf{G}_{\mathbf{1 3}}[\mathbf{G P a}]$ & 7.17 & 5.23 \\
$\alpha_{\mathbf{1}}\left[{ }^{\mathbf{O}} \mathbf{C}^{-\mathbf{1}}\right]$ & $-2.00 \mathrm{e}-08$ & $-1.00 \mathrm{e}-06$ \\
$\alpha_{\mathbf{2}}\left[{ }^{\circ} \mathbf{C}^{-1}\right]$ & $-3.27 \mathrm{e}-05$ & $-3.35 \mathrm{e}-05$ \\
$\mathbf{t}[\mathbf{m m}]$ & 0.2 & 0.2 \\
\hline
\end{tabular}

Table 3.2: Material Properties Used for AS4/8552 and TR50S-12K/NP301.

\subsection{Explanation of Research Design}

All laminates shapes studied were squares, that is the length equaled the width. Two materials were used for data extraction during this research with the intent of extracting trends in behavioral characteristics not purely dependent on materials. DA409/G35-150 and TR50S-12K/Newport 301 carbon fiber/epoxy resins were the prepreg unitape materials used for experiments. Since not all properties were found for DA409/G35-150, AS4/8552 was used as a substitute for property parameters for the analytical and finite element models due to the similar tensile $\left(E_{1}\right)$ and flexural $\left(E_{\text {bend }}\right)$ moduli (Table 3.1) $[2,7,9]$. The material properties used for modeling can be found in Table 3.2. Note that the thickness values $(t)$ found in Table 3.2 are the thickness per lamina after curing. The thickness per lamina of prepreg material is $t=0.12 \mathrm{~mm}$.

Analysis performed consisted of Hyers 1998 extended analytical model applied to thick bistable laminates, a finite element model performed in Abaqus CAE 6.14, and comparison with small sample experimentation to verify results. All laminates were modeled or fabricated with bistable layups, $\left[0_{k}, 90_{k}\right], k>0$, for $k$ layers until loss of bistability was found. The design parameters identified as crucial to a functional description were deflections, curvatures, energy (energy may be a discussion instead of a key parameter), snapthrough actuation force, and thickness to maintain bistability. 
Post-cure shape was determined by locating 11 equidistant nodes along the principle midline $\mathrm{x}$-axis and principle midline $\mathrm{y}$-axis of each laminate. Each of these nodes was then measured from a datum line, (the midplane of the undeformed pre-cure laminate), $z=0$, for the determination of outof-plane deflections. These out-of-plane deflections along the principle $\mathrm{x}$ and $\mathrm{y}$ axes can determine the principle paraboloid shapes of the laminates. This was achieved by using a domain for each midline from $-L / 2$ to $L / 2$ and using a fourth order bestfit polynomial to match each deflection shape.

Curvatures were derived from the deflection bestfit polynomials. Previously, there was another method used to determine the mean curvature of the laminate, essentially Menger curvature $[8,38]$.

$$
\kappa_{M}=\frac{1}{R}=\frac{4 A}{|x-y||y-z||z-x|}
$$

where $\mathrm{A}$ is the area of the triangle spanned by points $\mathrm{x}, \mathrm{y}$, and $\mathrm{z}$ (so long as the three points are colinear). This Menger curvature is the inverse of the radius $(R)$ of a circle whose equation is fitted to three measured points along the laminate span. This can be done piecewise iteratively for three nodes across the principle axes, or using the centroid and two edges. Another method to calculate curvature was parametrically using the function $(y)$ that bestfit the deflections:

$$
\kappa_{P}=\frac{y^{\prime \prime}}{\left(1+y^{\prime 2}\right)^{3 / 2}}
$$

It was determined through a small internal parametric study that the parametric curvature was more ideal and produced a smaller sum squared error when the curvature was not equivalent to a perfect circle. Otherwise, the Menger curvature proved to sufficient. In this work, parametric curvatures were used for laminates that were bistable in order to provide values along any point of either principle axis while the Menger curvature was used on nonbistable laminates or when enough data was unobtainable. 


\subsubsection{Analytical Model}

Hyer discussed an extended and updated version of his original analytical approach which was based on the Rayleigh-Ritz minimization of the potential strain energy within the laminate. His updated model changed basis from an assumed out-of-plane displacement paraboloid (Eq.3.3) to assumed strain polynomials in the principle $\mathrm{x}$ and $\mathrm{y}\left(\epsilon_{x}\right.$ and $\left.\epsilon_{y}\right)$ in Eq. 3.4-3.5 [5, 14, 24]:

$$
w^{0}(x, y)=\frac{1}{2}\left(w_{20} x^{2}+w_{02} y^{2}+w_{11} x y\right)
$$

where the coefficients are unknown constants with subscripts indicating the power of the $\mathrm{x}$ or $\mathrm{y}$ independent variable $[5,24]$. The $x^{2}$ and $y^{2}$ coefficients describe their respective curvatures while the $x y$ coefficient describes twist. According to Hyer's updated model, the mid-surface inplane strains are assumed to be:

$$
\begin{aligned}
& \epsilon_{x}^{0}=\epsilon_{x 00}+\epsilon_{x 20} x^{2}+\epsilon_{x 11} x y+\epsilon_{x 02} y^{2} \\
& \epsilon_{y}^{0}=\epsilon_{y 00}+\epsilon_{y 20} x^{2}+\epsilon_{y 11} x y+\epsilon_{y 02} y^{2}
\end{aligned}
$$

Thus, the approach undertaken was to use Eq. 3.4-3.5 to analyze a thick bistable square laminate by determining the total potential energy contained within the plate and calculating the resultant stable shapes via a minimization (first variation) of potential energy. For this scenario, the total potential energy of the plate is equivalent to the internal mechanical and thermal strains. In addition, since it is known that classical lamination theory does not adequately capture the cured shape, nonlinear terms were included within the strains in order to adequately capture the stable equilibrium behavior $[7,11,14,19]$.

The general equation of potential energy for the plate is the integral of its strain energy density, with thermal considerations, over the plate volume $[4,11,39]$ :

$$
W=\frac{1}{2} \int_{V}(C \epsilon-\epsilon \alpha \Delta T) d V
$$

where $\epsilon$ is the strain, $\alpha$ is the thermal expansion coefficient, $C$ is the stiffenss matrix, and $\Delta T$ is the change in temperature between the maximum fabrication temperature and final temperature after 
cooldown $\left(\Delta T=120^{\circ} C\right)$. In tensor form:

$$
W=\frac{1}{2} \int_{V}\left(\epsilon^{\prime} C \epsilon-\epsilon^{\prime} \alpha \Delta T\right) d V
$$

Using the plane stress assumption, Eq. 3.7 becomes

$$
W=\frac{1}{2} \int_{-L_{x} / 2}^{L_{x} / 2} \int_{-L_{y} / 2}^{L_{y} / 2}\left(\epsilon^{\prime} C \epsilon-\epsilon^{\prime} \alpha \Delta T\right) d x d y
$$

where $L$ is the plate sidelength. According to CLT, the stiffness matrix $C$ can be written as the $A B B D$ matrix. In addition, the thermal energy density, $\epsilon^{\prime} \alpha \Delta T$, will be represented as the strain vector, $\epsilon$, multiplied by the thermal force resultant, $N^{t h}$, and thermal moment resultant, $M^{t h}$, vector, such that Eq. 3.8 now becomes

$$
W=\frac{1}{2} \iint\left(\left[\begin{array}{l}
\epsilon^{0} \\
\kappa
\end{array}\right]^{\prime}\left[\begin{array}{ll}
A & B \\
B & D
\end{array}\right]\left[\begin{array}{l}
\epsilon^{0} \\
\kappa
\end{array}\right]-\left[\begin{array}{l}
\epsilon^{0} \\
\kappa
\end{array}\right]^{\prime}\left[\begin{array}{l}
N^{t h} \\
M^{t h}
\end{array}\right]\right) d x d y
$$

where $\epsilon$ consists of the mid-plane strains $\left(\epsilon^{0}\right)$ and the curvatures $(\kappa)$. Equations 3.10-3.12 defines the ABBD stiffness matrix typical of composite theory:

$$
\begin{aligned}
A_{i j} & =\sum_{k=1}^{N} Q_{i j}^{k}\left(z_{k}-z_{k-1}\right) \\
B_{i j} & =\frac{1}{2} \sum_{k=1}^{N} Q_{i j}^{k}\left(z_{k}^{2}-z_{k-1}^{2}\right) \\
D_{i j} & =\frac{1}{3} \sum_{k=1}^{N} Q_{i j}^{k}\left(z_{k}^{3}-z_{k-1}^{3}\right)
\end{aligned}
$$

where $Q^{k}$ is the reduced stiffness matrix of the $k$ th layer of the composite ( $n$ layers) and $z_{k}$ is the location along the z-axis of the bottom of a lamina and $z_{k-1}$ is the location of the top of the lamina $[6,7,10] . N^{t h}$ and $M^{t h}$ are defined in Eq. $3 \cdot 13-3.14$ as

$$
\begin{aligned}
N_{i}^{t h} & ==\sum_{k=1}^{N} \sum_{k=1}^{N} Q_{i j}^{k} \alpha_{j}^{k}\left(z_{k}-z_{k-1}\right) \Delta T \\
M_{i}^{t h} & ==\frac{1}{2} \sum_{k=1}^{N} \sum_{k=1}^{N} Q_{i j}^{k} \alpha_{j}^{k}\left(z_{k}^{2}-z_{k-1}^{2}\right) \Delta T
\end{aligned}
$$


In order to get the out-of-plane strains, the associated $x$ and $y$ mid-plane displacements have to be solved as a function of the assumed strains. The inplane displacements are:

$$
\begin{aligned}
& u^{0}=\int\left[\epsilon_{x}^{0}-\frac{1}{2}\left(\frac{\partial w^{0}}{\partial x}\right)^{2}\right] d x+u_{01} y+u_{03} y^{3} \\
& v^{0}=\int\left[\epsilon_{y}^{0}-\frac{1}{2}\left(\frac{\partial w^{0}}{\partial y}\right)^{2}\right] d y+v_{01} y+v_{03} y^{3}
\end{aligned}
$$

Thus, the mid-plane strain vector with the inclusion of von-Karmin nonlinear terms is

$$
\epsilon^{0}=\left[\begin{array}{c}
\epsilon_{x}^{0} \\
\epsilon_{y}^{0} \\
\epsilon_{x y}^{0}
\end{array}\right]=\left[\begin{array}{c}
\frac{\partial u^{0}}{\partial x}+\frac{1}{2}\left(\frac{\partial w^{0}}{\partial x}\right)^{2} \\
\frac{\partial v^{0}}{\partial y}+\frac{1}{2}\left(\frac{\partial w^{0}}{\partial y}\right)^{2} \\
\frac{1}{2}\left(\frac{\partial u^{0}}{\partial y}+\frac{\partial v^{0}}{\partial x}+\frac{\partial w^{0}}{\partial x}\left(\frac{\partial w^{0}}{\partial y}\right)\right.
\end{array}\right]
$$

and the mid-plane curvatures are defined as

$$
\kappa=\left[\begin{array}{c}
\kappa_{x} \\
\kappa_{y} \\
\kappa_{x y}
\end{array}\right]=\left[\begin{array}{c}
-\frac{\partial^{2} w^{0}}{\partial x^{2}} \\
-\frac{\partial^{2} w^{0}}{\partial y^{2}} \\
-\frac{\partial^{2} w^{0}}{\partial x \partial y}
\end{array}\right]
$$

The total strain is then the sum:

$$
\epsilon=\epsilon^{0}+z \kappa
$$

Additionally, to eliminate motion in the x-y plane of the laminate, the linear terms in Eq. 3.15-3.16 are set equal to one another [5].

Plugging in the displacement equations into the strain and curvature relations, then inserting into the total potential energy (Eq. 3.9) along with Eq. 3.10-3.14 develops a functional describing the laminate's potential energy. Integrating this functional over the region $\frac{-L_{x}}{2}<x<\frac{L_{x}}{2}, \frac{-L_{y}}{2}<$ $y<\frac{L_{y}}{2}, n$ plies, yields the nonlinear polynomial functional $W$ as a function of the 15 unknowns established in Eq. 3.3-3.5 and Eq. 3.15-3.17. Minimizing the first variation of the resultant functional yields 15 nonlinear equations which can be used to solve for the 15 unknowns parameters of the system. These parameters can then be used to solve for the energy of the stable configurations at the time of post-cure. 
This system of unknown parameters was obtained with MATLAB's symbolic package, and the parameters were solved numerically with the aid of Wolfram Mathematica's solve function. After the parameters were calculated, they were plugged back into the resultant functional to determine the total potential energy of the laminate. Additionally, the parameters $w_{20}, w_{02}$, and $w_{11}$ were inserted into Eq. 3.3 to determine the out-of-plane displacement and curvatures.

\subsubsection{Finite Element Analysis}

A 3-dimensional laminated composite shell model was created in Abaqus CAE 6.14. All FEA laminate models were $0.914 \mathrm{~m} \times 0.914 \mathrm{~m}$ using $0.2 \mathrm{~mm}$ thickness. The solution technique used for each STEP was a nonlinear Newtonian with Abaqus Standard/CAE. Mesh size was determined by an iterative process of decreasing subsequent mesh size by 50 percent and comparing the results of the previous larger mesh size. If the results of the smaller mesh size were identical, then the mesh size was considered adequate and converging. For most laminates, the mesh size used resulted in 1600 four-node square reduced integration (S4R) elements.

Primary cure shape was calculated via a cooling STEP from a simulated oven temperature down to room temperature $\left(\Delta T=121^{\circ} C\right)$. Secondary cure shape was determined by holding nodes on the edge of the midline y-axis while a sufficient out-of-plane transverse load was applied to actuate snapthrough. The load and edge boundaries were removed, and the centroid fixed. The process was applied again to cause snapback except the midline x-axis edges were held fixed and the load was applied in the negative z-direction. Loading steps used a fixed damping factor of 2.5e-07. An energy versus time plot was generated to show the energy landscape for the simulation and check for bistability. At this point, it was known that global minima at $t=1$ second (end of the post cure step), $t=3=5$ seconds (end of snapthrough and snapback loadings) in the energy plot indicated stable configurations.

The snapthrough actuation loads were determine separately in a different process. The post-cure shape was generated in the same method mentioned above. Then the laminate was clamped/fixed on an edge area of 23mm line along the midline y-axis (Fig. 3.1) while an outof-plane transverse load was applied along the positive z-axis to the centroid on an approximate area $45 \mathrm{~mm} \times 45 \mathrm{~mm}$. This force was a ramp linear increase until the laminate "snapped" secondary stable configuration. A similar procedure was performed for the snapback force except that the midline $\mathrm{x}$-axis edges are clamped and the force is applied in the negative $\mathrm{z}$ direction. Snapthrough 


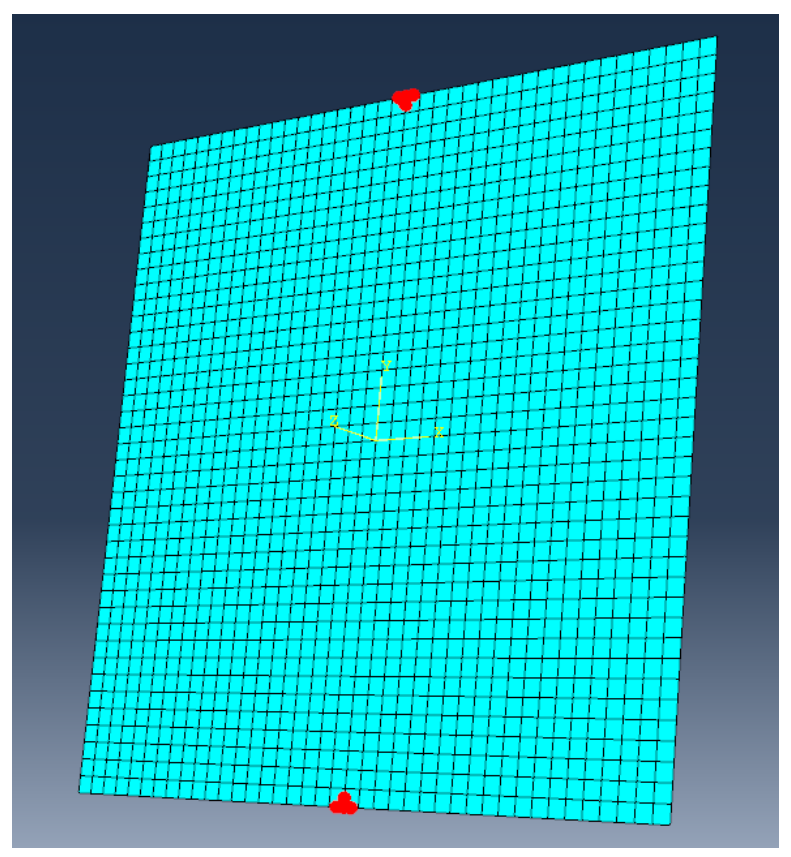

Figure 3.1: Y and -Y FEA Boundary Conditions Highlighted on the Mesh for Determining Snapthrough Actuation Load.

forces were found by plotting the applied load versus the centroid node displacement. During the loading period, if there existed an extremely large displacement for a given load value, this indicated the snapthrough $[7,8]$. Detailed descriptions of the procedures follows.

\subsubsection{Finite Element Analysis Simulation Instructions}

1. Creating the Part Geometry:

a. Open Abaqus and select "With Standard/Explicit Model" under "Create Model Database" contained within the prompt window.

b. Creating the geometry is done by right clicking "Parts" section on the left-hand size of the screen. Here you can name the specific part. Options that need to be selected are: "3D, Deformable, Shell, Type: Planar."

c. Draw the geometry using tools in the Toolbox area. For this research, a $914 \mathrm{~mm}$ x $914 \mathrm{~mm}$ square was created and dimensioned.

d. Next define material properties by right clicking and "Create" under the "Materials" tab in the left tech tree. The options selected are "Type: Mechanical, Elasticity: Elastic, 


\begin{tabular}{|l|c|c|}
\hline & AS4/8552 & TR50S-12K/NP301 \\
\hline \hline $\mathbf{E}_{\mathbf{1}}[\mathbf{G P a}]$ & 13500 & 14200 \\
$\mathbf{E}_{\mathbf{2}}[\mathbf{G P a}]$ & 9500 & 9000 \\
$\nu_{\mathbf{1 2}}[-]$ & 0.3 & 0.29 \\
$\mathbf{G}_{\mathbf{1 2}}[\mathbf{G P a}]$ & 5000 & 7850 \\
$\mathbf{G}_{\mathbf{2 3}}[\mathbf{G P a}]$ & 3970 & 5230 \\
$\mathbf{G}_{\mathbf{1 3}}[\mathbf{G P a}]$ & 7170 & 5230 \\
$\alpha_{\mathbf{1}}\left[{ }^{\mathbf{O}} \mathbf{C}^{-\mathbf{1}}\right]$ & $-2.00 \mathrm{e}-08$ & $-1.00 \mathrm{e}-06$ \\
$\alpha_{\mathbf{2}}\left[{ }^{\circ} \mathbf{C}^{-\mathbf{1}}\right]$ & $-3.27 \mathrm{e}-05$ & $-3.35 \mathrm{e}-05$ \\
$\mathbf{t}[\mathbf{m m}]$ & 0.12 & 0.12 \\
\hline
\end{tabular}

Table 3.3: Material Properties Used for AS4/8552 and TR50S-12K/NP301 during FEA.

Type Lamina." Material properties are then entered in Megapascals to remain consistent with "mm."

e. Additionally another Material Behavior was added by selecting in the same window "Type: Mechanical, Expansion, Type: Orthotropic." The thermal expansion properties were entered as from Table 3.3 with $\alpha_{3}=\alpha_{2}$.

Note that Abaqus requires users to maintain their own consistency with units. The numbers entered for Material 1: "DA409" can be found in Table 3.3. Steps $\mathbf{d}$ and e were repeated to create a second material labeled as "TR50S" whose data values were entered exactly as presenting in Table 3.3 .

f. The layup scheme was created for a composite material by right-clicking the "Sections" in the model tree, giving an appropriate name relative to the layup scheme to be made, and choosing the options "Category: Shell, Type: Composite." This window will close and new window will automatically open were the layup needs to be created. Here you can choose the Material type per lamina, specify the thickness, orientation angle per lamina, integration points and name of each lamina. Each lamina was named as a number thickness specified as $0.2 \mathrm{~mm}$. The first half of all lamina were given the same orientation angle of 0 degrees while the second half were given the orientation angle of 90 degrees. Note that the last row corresponds to the bottom ply and the first row corresponds to the top ply. A "section" was created for each layup being simulated and for each material. For example, "10plyDA409" and "10plyTR50" sections were made.

2. Creating "sets," assigning the material, and mesh. 


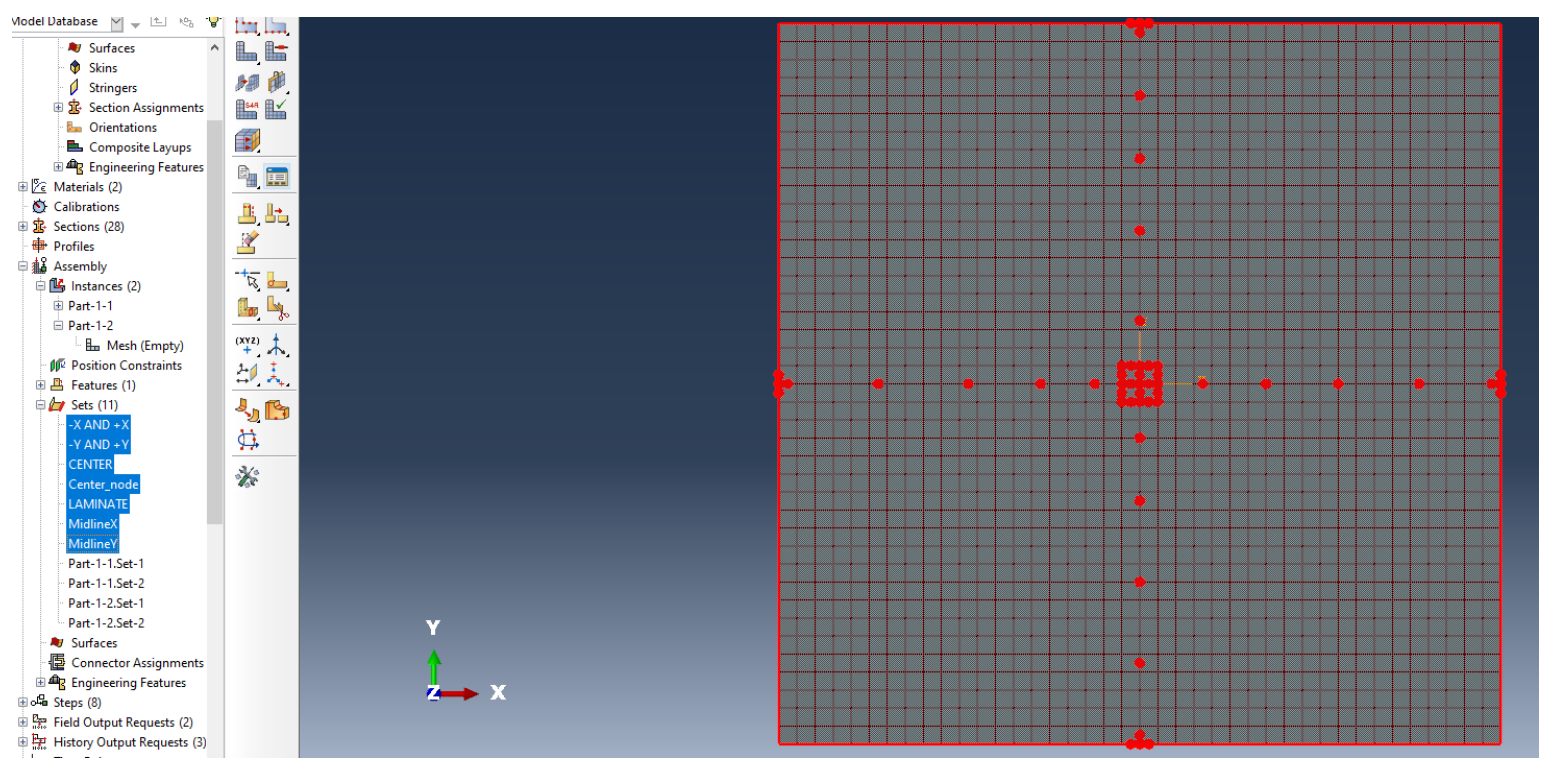

Figure 3.2: Nodes Contained in Sets are Highlighted in Red Indicating Locations of Loads and Boundary Conditions.

Sets are used to specify specific points of interest on the lamina. Sets should be made after the mesh has been created to allow nodes to be used as specific points of interest. If specific regions of the laminate are of interest then it must be partitioned. Partitions were not used explicitly in this research but can be used to assign different mesh types to different regions.

a. Partitions are created by choosing in the toolkit area "Partition Face, Sketch."

b. The mesh is created by opening the "Assembly" subtree in the Model tree area. Right-click "Instance" and create a new instance. Choose the Part that you intend to use, and "Instance Type: Independent." Click "Ok."

c. Next an element type must be chosen. This is done by going to Assembly $\rightarrow$ Instance $\rightarrow$ Part 1-1 $\rightarrow$ mesh(empty). Next choose the "Assign Element Type" in the toolbox area. The options selected were "Standard, Geometric Order: Linear, Second Order Accuracy: Yes."

d. Next select "Seed Part Instance" in the toolbox area. Select global seed size as 23. Then selected the "Mesh Part" in the toolbox area, meshing the entire laminate.

e. Sets are created under Assembly $\rightarrow$ Sets $\rightarrow$ right-click sets and create. Sets were created for specific areas. The following sets were created (Figure 3.2) where specific sets were created for the midline of the $\mathrm{x}$-axis, the midline of the $\mathrm{y}$-axis (both with approximately equidistantly 


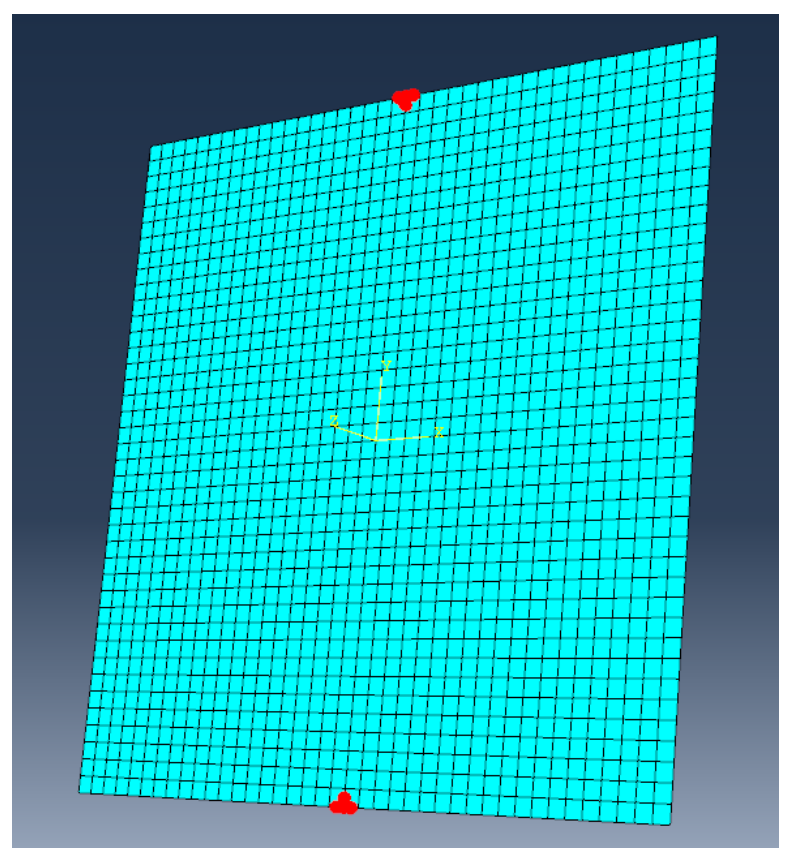

Figure 3.3: Cooling Boundary Conditions for Initial Step.

chosen nodes), the centroid node, a centroid node area, the center edge nodes as a single set for $\mathrm{X}$ and $-\mathrm{X}$ axis, the center edge nodes as a single set for the $\mathrm{Y}$ and $-\mathrm{Y}$ axis, and loading areas for clamping positions in the same locations.

f. Material sections are applied under the "Model $\rightarrow$ Part $\rightarrow$ Section Assignment." Section Assignment is right-clicked and "create" is chosen. Choose "Section" of interest, from "middle surface," then select the "Create new Set" and select the entire laminate.

3. Option A: Simulation Steps, Loads and Boundary Condition Assignment for Cure Shape Calculations.

This Option is for creating a simulation to obtain both cure shape configurations. This simulates cooling of the laminate from elevated oven temperature down to room temperature. Then the laminate is "snapped" from the primary cure-shape configuration to the secondary cure-shape configuration. There are multiple methods to doing this including the "Static:Riks" method. This option utilizes "Static: General" method.

a. For every step, a boundary condition (BCs) is defined. A simulation step is created by right-clicking "Steps" and selecting "Create." However, here the "Initial Step" is propagated. 


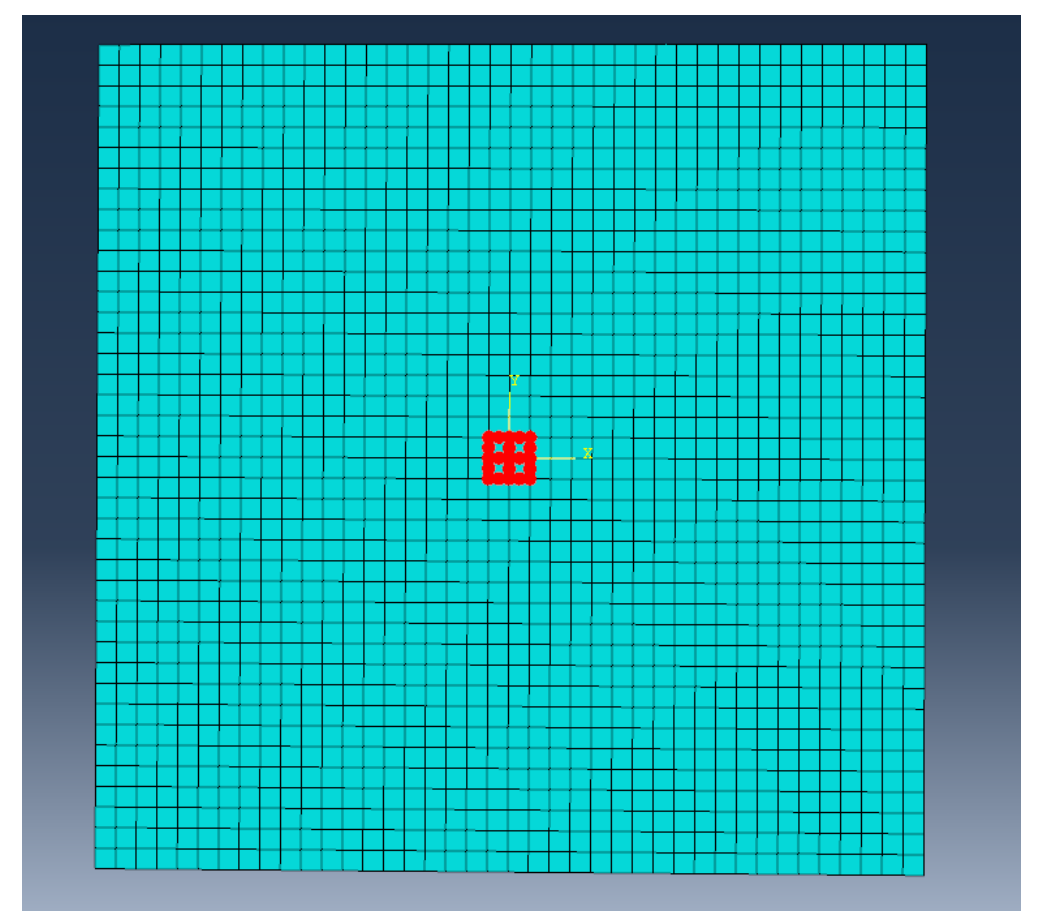

Figure 3.4: FEA Mesh with Highlights of the Loading Area Used for Determining Snapthrough Actuation Loads.

A BC is created by right-clicking "BC" under the initial step, labeling it, and selecting "Category: Mechanical, Type: Displacement/Rotation." The "Center_Node" set is selected with all displacements and rotations set equal to zero (Figure B.2).

b. Cooling: Create another new step, "Name: Cooling, Procedure Type: Static, General." Click continue and select "Nlgeom." Click on "Predefined Field: Status: Modified, Magnitude: 20." The BCs are propagated fro the previous step.

c. Snapthrough Loading Step: Create a new step, "Name: STL, Procedure Type: Static, General." Select automatic stabilization: "Specify Damping Factor: 2.5e-007." Click Ok. Deactivate "BC-1." Assign a boundary condition for the snapthrough load by right-clicking "BC" and selecting "create" BC $\rightarrow$ "Mechanical, Displacement/Rotation" $\rightarrow$ Select "Y and -Y." Assign $U 3=U R 1=U R 2=U R 3=0$ allowing the laminate to mobility in the XY plane. Finally create the loading condition by right-clicking "Loads" and creating a new load "STL_Load" $\rightarrow$ Mechanical, Concentrated Force $\rightarrow$ Select Set "Center_Node" $\rightarrow C F 3=500$ for 500 Newtons. Note that this load is applied in the positive z-direction. Also, as thicknesses 
increase, Abaqus may not converge, thus the remedy being to increase this load to cause snapthrough.

d. Snapthrough Unloading Step: Create a new step $\rightarrow$ "Name: STU, Procedure Type: Static, General" $\rightarrow$ Continue $\rightarrow$ Ok. Deactivate the current BCs. Create a new BC $\rightarrow$ "Mechanical, Displacement/Rotation" $\rightarrow$ Continue $\rightarrow$ Select set "Center_Node." Assign $U 1=U 2=U 3=$ $U R 1=U R 2=U R 3=0$ such that the laminate center is held fixed and the edges can be released from load stress. Finally, deactivate "STL_Load."

e. Snapback Loading Step: Create a new step $\rightarrow$ "Name: SBL, Procedure Type: Static, General" $\rightarrow$ Select automatic stabilization: "Specify Damping Factor: 2.5e-007" [29]. Click Ok. Deactivate BC-1, BC-2, and BC-3. Create a new BC $\rightarrow$ "Mechanical, Displacement/Rotation" $\rightarrow$ Select "X and -X." Assign $U 3=U R 1=U R 2=U R 3=0$ allowing the laminate to move in the XY plane. Apply a new load $\rightarrow$ "Loads" $\rightarrow$ "Mechanical, Concentrated Force" $\rightarrow$ Continue $\rightarrow$ Select "Center_Node" $\rightarrow C F 3=-500$ acting in the negative z-direction.

f. Snapback Unloading Step: Create a new step $\rightarrow$ "Name: SBU, Procedure Type: Static, General" $\rightarrow$ Continue $\rightarrow$ Ok. Deactivate the current BCs. Create a new BC $\rightarrow$ "Mechanical, Displacement/Rotation" $\rightarrow$ Continue $\rightarrow$ Select set "Center_Node. Assign $U 1=U 2=U 3=$ $U R 1=U R 2=U R 3=0$ such that the laminate center is held fixed and the edges are free. Finally, deactivate "STB_Load."

3. Option B: Simulation Steps for Generating Snapthrough Forces. Option B applies the load to an centroidal area versus the concentrated point load in Option A.

a. For every step, a boundary condition (BCs) is defined.A simulation step is created by right-clicking "Steps" and selecting "Create." However, here the "Initial Step" is propagated. A BC is created by right-clicking "BC" under the initial step, labeling it, and selecting "Category: Mechanical, Type: Displacement/Rotation." The "Center" set is selected with all displacements and rotations set equal to zero. (Figure B.2).

b. Cooling: Create a new step, "Name: Cooling, Procedure Type: Static, General." Click continue and select "Nlgeom." Click on "Predefined Field: Status: Modified, Magnitude: 20." The BCs are propagated from the previous step.

c. Snapthrough Loading Step: Create a new step, "Name: STLe1, Procedure Type: Static, 
General." Select automatic stabilization: "Specify Damping Factor: 2.5e-007." Click Ok. Deactivate "BC-1." Assign a boundary condition for the snapthrough load by right-clicking "BC" and selecting "create" BC $\rightarrow$ "Mechanical, Displacement/Rotation" $\rightarrow$ Select "Y and -Y." Assign $U 3=U R 1=U R 2=U R 3=0$ allowing the laminate to mobility in the XY plane. Finally create the loading condition by right-clicking "Loads" and creating a new load "STL_Load" $\rightarrow$ Mechanical, Concentrated Force $\rightarrow$ Select Set "Center" $\rightarrow C F 3=500$ for 500 Newtons. Note that this load is applied in the positive z-direction. Also, as thicknesses increase, Abaqus may not converge, thus the remedy being to increase this load to cause snapthrough.

5. Data Collection was performed through the use of the "field output request" and "history output request." New requests were made within Abaqus to record information during all simulation steps for the displacement of both midline axes and centroid node, internal strain energy, and applied load.

6. Job creation: Right-click "Job" and "Create" a new Job. Jobs were named for their material and thickness and lamina size. Multiple processors were used when available. Right-click on the Job name and select "Submit." After the Job has been completed, results can be found by Job $\rightarrow$ "Results."

\subsubsection{Experimentation}

Experiments were desired to assess the validity of analytical and FEM approaches. DA409 and TR50S prepreg unitape material was used. Square $0.325 \mathrm{~m} \times 0.325 \mathrm{~m}$ samples of DA409 and TR50S were fabricated at $6,8,10$, and 12 ply thicknesses. $0.965 \mathrm{~m} \times 0.965 \mathrm{~m}$ samples of $4,14,18,28$, and 36 plies were fabricated with DA409 while 10, 20, 28, 30, and 36 ply samples were fabricated with TR50S. Samples were purposefully made slightly larger, by approximately $25 \mathrm{~mm}$ on each side, to have more area to clamp and in order to minimize influence of the clamps on bistable behavior. Hand layup and vacuum bagging techniques were used to assemble in the unsymmetric crossply [0/90] pattern. The mold used was a 6061-T6 aluminum 5mm plate sized to fit into the oven. One to five layers of FibRelease 1153 mold release, nylon release peel ply, high temperature nonperforated release ply, Airweave breather fabric, and Stretchlon vacuum bagging were used. One layer of nylon peel ply went between the mold and the laminate and another single layer was laid 


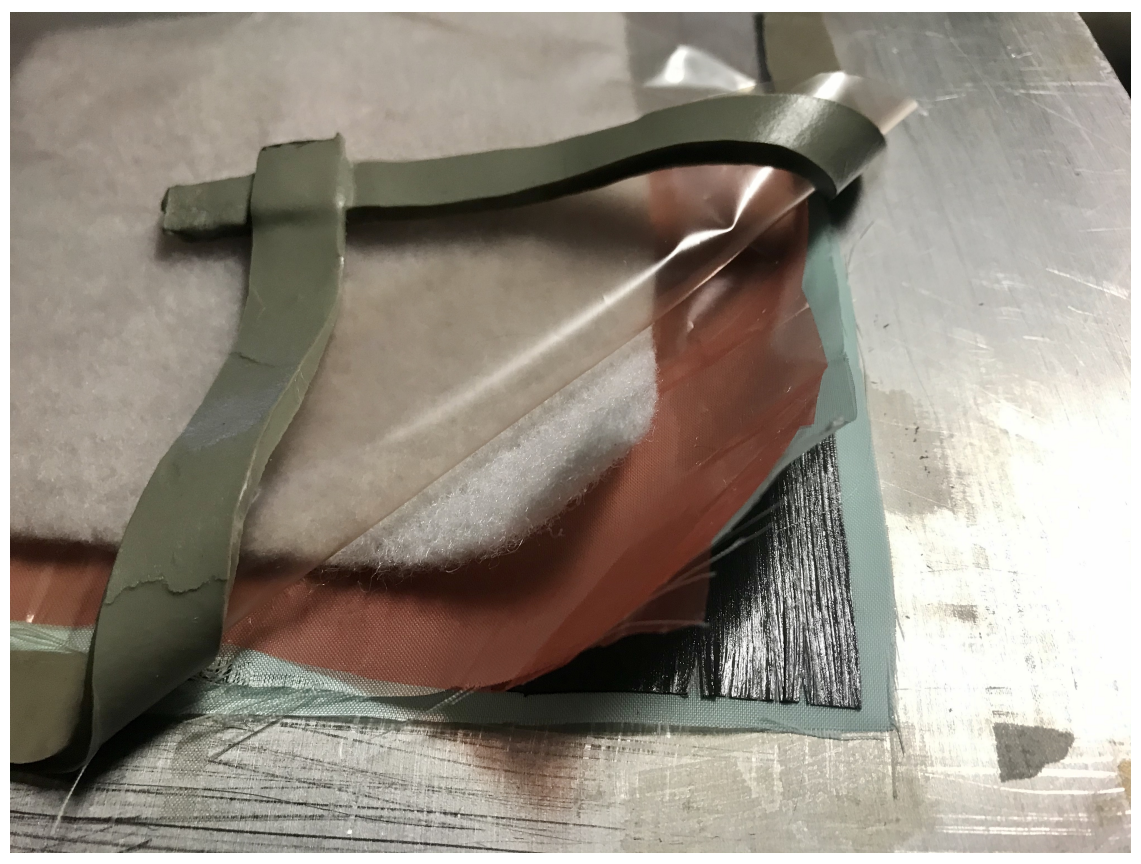

Figure 3.5: Vacuum Bag Opened up to Show Layup with Green Peel Ply, Red Peel Ply, Breather, and Transparent Vaccum Bag Sealed with Mastic Tape.

directly over top of the carbon fiber, followed by the non-perforated release ply, breather fabric, and vacuum bag. DA409 came in $305 \mathrm{~mm}$ wide prepreg unitape sheet rolls that were $0.12 \mathrm{~mm}$ thick and TR50S came in $1016 \mathrm{~mm}$ wide prepreg unitape sheet rolls that were $0.12 \mathrm{~mm}$ thick (per manufacturer). Samples were cured in an oven heated to $140^{\circ} \mathrm{C}$ for 90 minutes then left to cool to room temperature $\left(\Delta T=121^{\circ} C\right)$. Some samples were pulled out of the oven to cool while others were left in the oven.

The cured samples were each tested for the existence of bistable shape morphing by applying loads to observe snapthrough. Bistable samples' deflections were recorded using 11 nodes along the midlines while non-bistable samples had their peak deflections recorded. Snapthrough actuation loads were tested in simply supported configurations on a cleared flat surface with a $25 \mathrm{~mm}$ diameter point loads (Figure 3.4). Additionally, snapthrough actuation loads were tested by clamping the middle of two opposite edges, on a $25 \mathrm{~mm} \times 25 \mathrm{~mm}$ wide area, to a stand in accordance to previously discussed boundary conditions (Fig. 3.5). 4.0mm holes were drilled into the centroid in order to fit a high tensile strength cord to attach weight from below. A bucket was attached to the cord with a force scale. Water was continuously added at a slow rate until the laminates snapped to their secondary stable shape. 


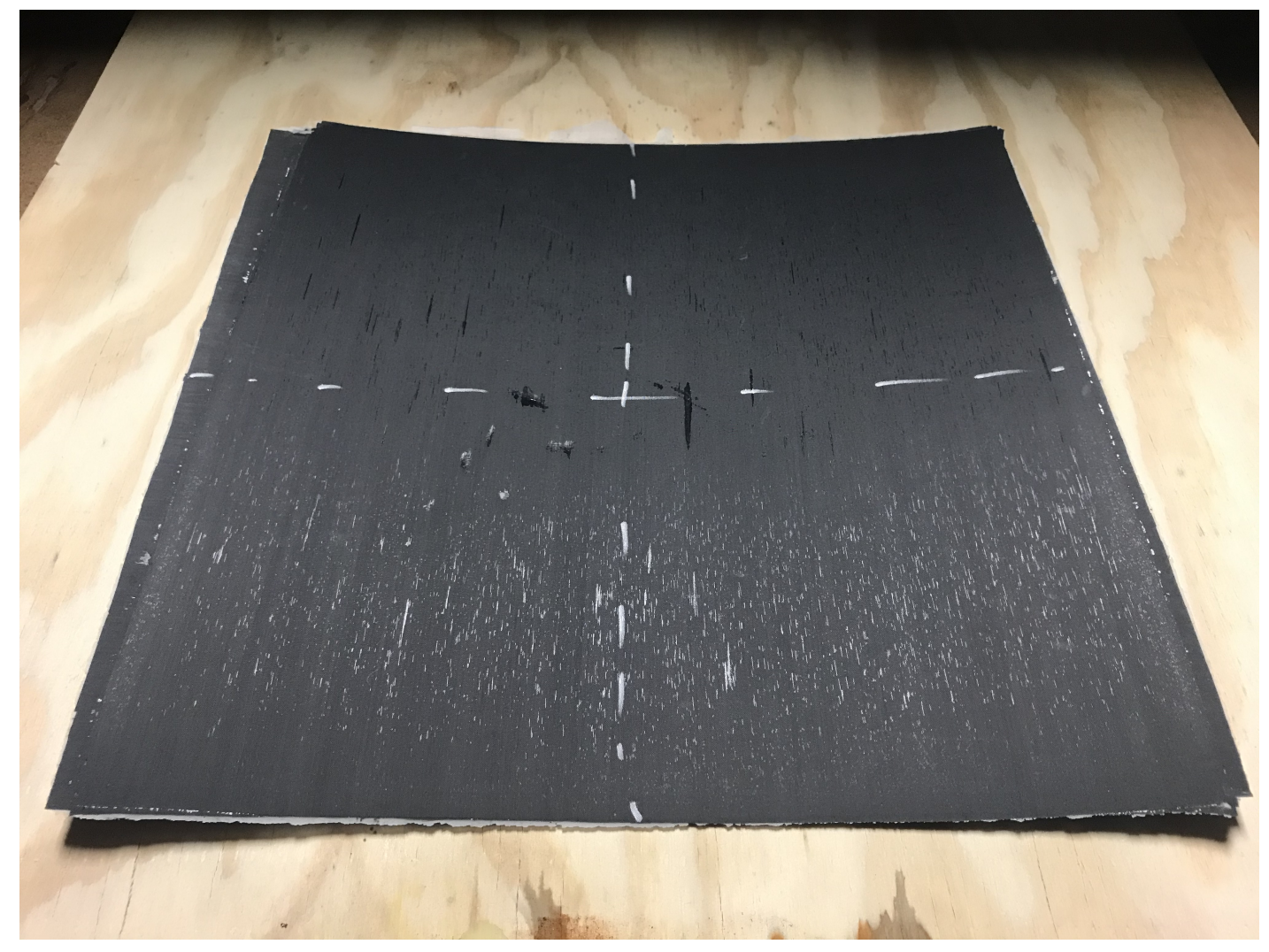

Figure 3.6: Simply Supported Loading Condition Experiments.

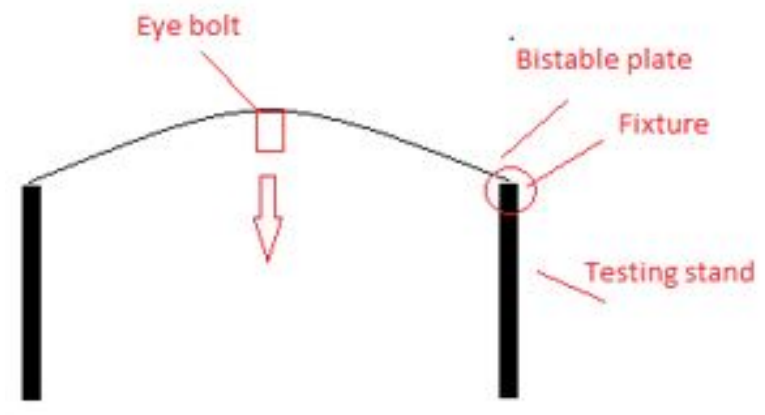

Figure 3.7: Clamped Fixture Support Diagram for Experiments. Eye bolt was the Location of Applied Load. 
Post analysis for all methods was performed in MATLAB by determining the peak centroidal displacement for each model to until just after loss of bistability occurred. These displacements were plotted against sidelength/thickness aspect ratio to create bifurcation plots. Addition information can be found in the appendices.

\subsection{Justification of the Methodological Decisions and Their Limits}

Both a physics based analytical and finite element model were chosen to provide descriptions for the cure-shapes of the laminate for a couple of reasons. The first is that research has pointed towards the idea that FEA is able to generate better descriptions of the cure shape for all regions of the laminate, including edges whereas analytical approaches have only successfully determined generalized shape descriptions. Second, there should be a measurable difference between the two approaches. Additionally the analytical model uses a plane stress assumption to simplify calculations while FEA uses a 3D shell analysis. It is one of the aims of this research to provide quantifiable merit to both methods in determining cure shape. Finally, one method may provide better insight to describe laminates whose geometric characteristics lend themselves to being close to the critical bifurcation point.

The primary post-cure shape was determined through a standard cooldown procedure as it was the simplest. Secondary cure shape was done through an applied loading to see if the laminate would snapthrough or deform plasticly. Snapthrough loadings had to be performed in a different procedure as single nodes were used for the load and boundary conditions for cure shape determinations. As such, a single node point load would likely result in unrealistically high snapthrough loads hence the area increase for solving actuation snapthrough loads. This area is comparable to the area in experiments in the simply supported scenario.

Experiments were performed for samples of two different materials and sizes to provide validation of nondimensional determinations. Sample snapthrough behavior was explored through both clamped and simply supported boundary conditions. Simply supported BCs were used to determine baseline while clamped BCs were used to replicated a two-point attachment system, as if the laminate was apart of a panel system. 


\subsection{Summary of Methods}

The research methodology is designed to explore and identify key characteristics of bistable laminates. Firstly, use Rayleigh-Ritz, FEA, and experiments to determine a description of the critical point of bistability as related to Phatak's length-to-ply 1.2:1 ratio $\left(L_{\text {critical }}^{*}=152\right)$, namely, the extent of this ratio. Secondly, identify deflections and curvatures for thicker laminates in order to provide physical description of the post cure equilibrium shapes. Lastly, using both Rayleigh-Ritz and FEA to provide descriptions of the snapthrough behavior for thick laminates. 


\section{Chapter 4}

\section{Results and Implications}

This chapter details the results obtained from the Rayleigh-Ritz analytical model, finite element model, and experiments. Results will be broken down into the categories of cure shape analysis and snapthrough behavior. At the end of each category will be a comparison between modeling results and experimental findings. The chapter will conclude with a discussion for sources of error and a lamina thickness sensitivity study.

\subsection{Cure Shape}

\subsubsection{Analytical Model Cure Shape Predictions}

After generating the system of equations using MATLAB's symbolic package, the results of Hyer's extended analytical model were obtained through the use of Mathematica's solve function. This function proved to be very useful in its ability to find solutions for the system of 15 nonlinear equations. Generally, solve would generate anywhere from one to 10 satisfactory solutions. Due to the theoretical inability of square laminates to exhibit twist curvature, and since square laminates were the interest of this study, only solutions that had the value of $w_{11}=0$ were recorded. Samples that were less than the sidelength-to-thickness ratio of 152 were all expected to be bistable [9]. For these samples, there was always a minimum of three solutions generated. The solution values of all 15 parameters were then inputted into the resultant functional equation to solve for the total equilibrium potential energy value (Table 4.1). Potential energies of primary and secondary cure 


\begin{tabular}{|c|c|c|}
\hline \# of Plies & $W_{A S 4 / 8552}[\boldsymbol{J}]$ & $W_{T R 50 S-12 K / N P 301}[\boldsymbol{J}]$ \\
\hline \hline $\mathbf{4}$ & -3.56 & -4.68 \\
$\mathbf{1 0}$ & -8.9 & -11.7 \\
$\mathbf{1 4}$ & -12.5 & -16.5 \\
$\mathbf{1 8}$ & -16.2 & -21.3 \\
$\mathbf{2 0}$ & -18.2 & -23.7 \\
$\mathbf{2 8}$ & -26.8 & -34.2 \\
$\mathbf{3 0}$ & -29.4 & -34.2 \\
$\mathbf{3 6}$ & -38.6 & -37.1 \\
$\mathbf{4 0}$ & -45.3 & -47.0 \\
\hline
\end{tabular}

Table 4.1: Calculated Energy (Joules) of Stable Equilibrium Shapes Generated from Rayleigh-Ritz.

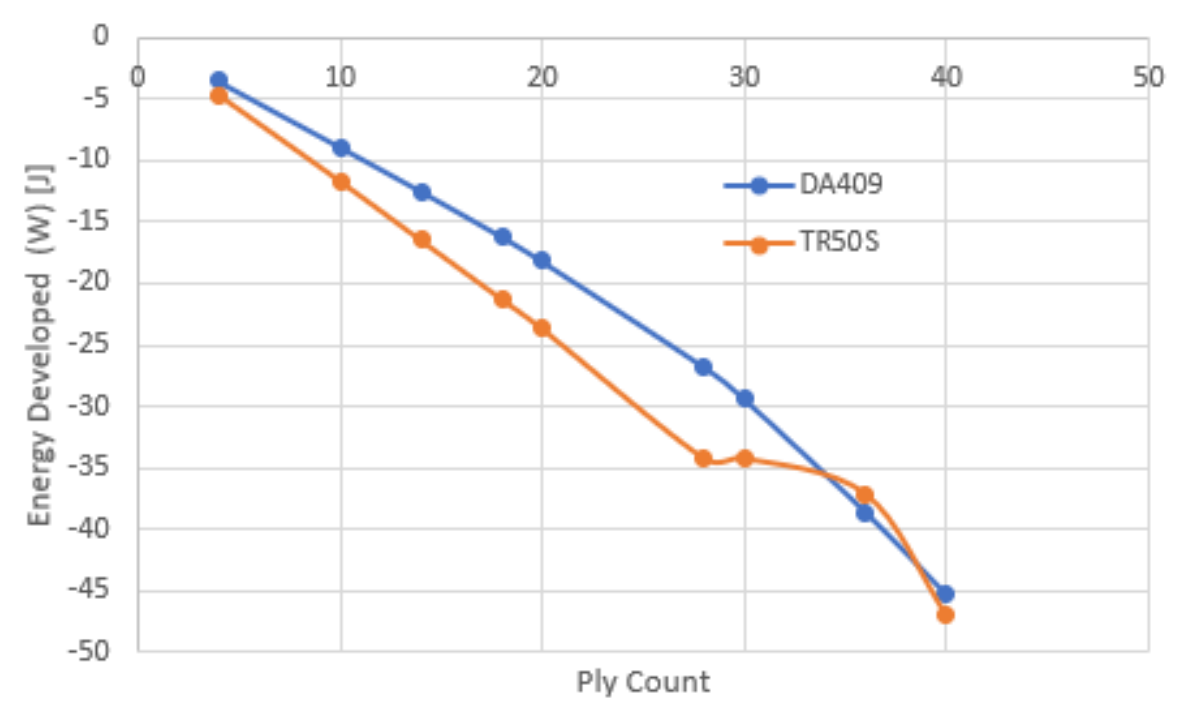

Figure 4.1: Energy Developed at Equilibrium Stable Configurations for Increasing Thickness

shapes were always equivalent.

In Table 4.1, as the sidelength-to-thickness decreased increased, the magnitude of energy developed for equilibrium positions generally increased. It was unsure if any specific trends were present until a plot was created (Figure 4.1).

Figure 4.1 shows an increasing trend in the magnitude of energy developed for both materials, especially for DA409. However, the energy developed for TR50S is equivalent at 28 and 30 plies. Here the curve flattens out then again continues increasing.

The parameters $w_{10}, w_{01}$, and $w_{11}$ were then inputed into a MATLAB script, that utilized the surf function, to visualize the primary and secondary configuration shapes. It was observed that two out of the three solutions for samples of sidelength-to-thickness ratio greater than 152 always 


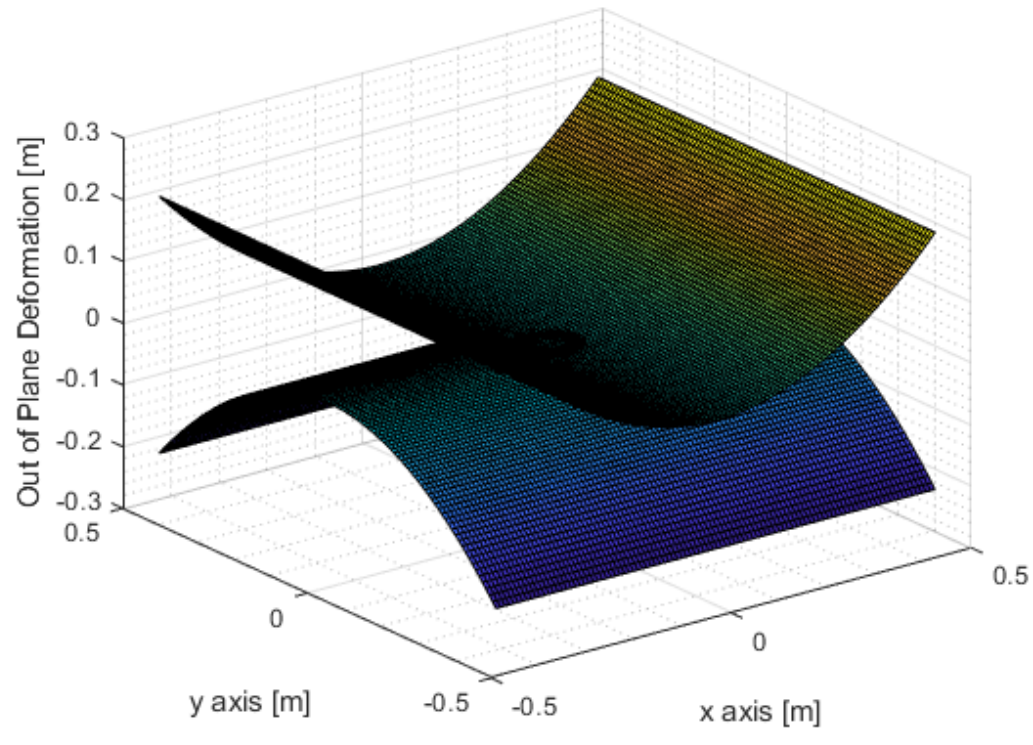

Figure 4.2: Visualizations of Cure Shape Stable Configurations for a 4-Ply DA409 Laminate from Rayleigh-Ritz

indicated primary and secondary stable shapes. These stable configurations were of equal and opposite axis curvature; that is, $\kappa_{x}$ of configuration 1 equaled $-\kappa_{y}$ of configuration 2 . Additionally, the remaining solution was a saddle shape which contained $\kappa_{x}=-\kappa_{y}$. Visualizations of the stable configurations for a 4-ply DA409 laminate can be seen in Figure 4.2. An observation found was that as laminate thickness increased, the edges went from being flat to parabolic in shape (Figure 4.3). However, this could simply be due to the generalized out-of-plane shape assumption. This also implies that the generalized out-of-plane shape polynomial may be more suited for describing thinner bistable plates and becomes less applicable to thick laminates.

For DA409 modeled laminates, when the sidelength-to-thickness ratio was 126.9, only one solution was generated by Mathematica. This solution had $\kappa_{x}=w_{10}=w_{01}=\kappa_{y}$ indicating a saddle shaped laminate, seen in Figure 4.3. Furthermore, the absence of additional solutions implied that bistability of the laminate was lost. Examination of TR50S laminates showed multiple solutions of interest for sidelength-to-ply ratios greater than one. It was not until the thickness reached 40 plies that there existed one solution for the respective system of equations. The solution was similar to the 36-ply DA409 case, in that $\kappa_{x}=-\kappa_{y}$.

The same MATLAB script used to gain visualizations of out-of-plane cure shapes was able 


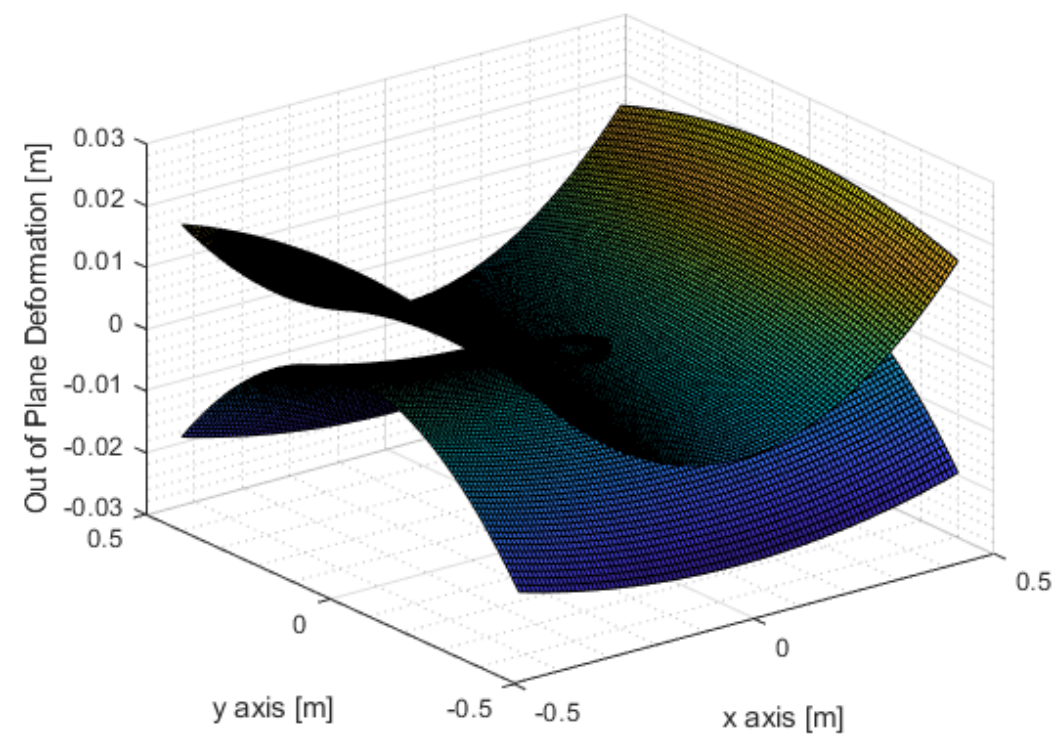

Figure 4.3: 30-Ply DA409 Rayleigh-Ritz Shape Cure Shape Prediction Using Matlab. Notice the Parabolic Edge Compared to the Flat Edge of Figure 4.2.

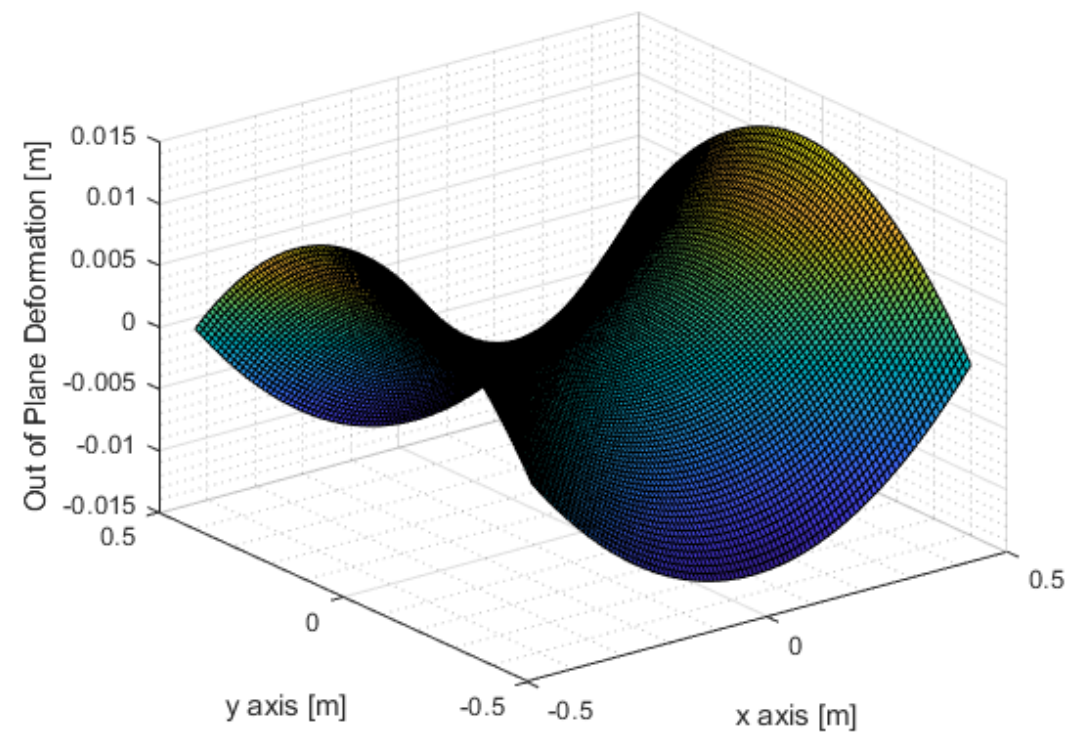

Figure 4.4: Saddle Shape Prediction for 36-Ply DA409 Laminate Generated by Rayleigh-Ritz Using Matlab surf. 


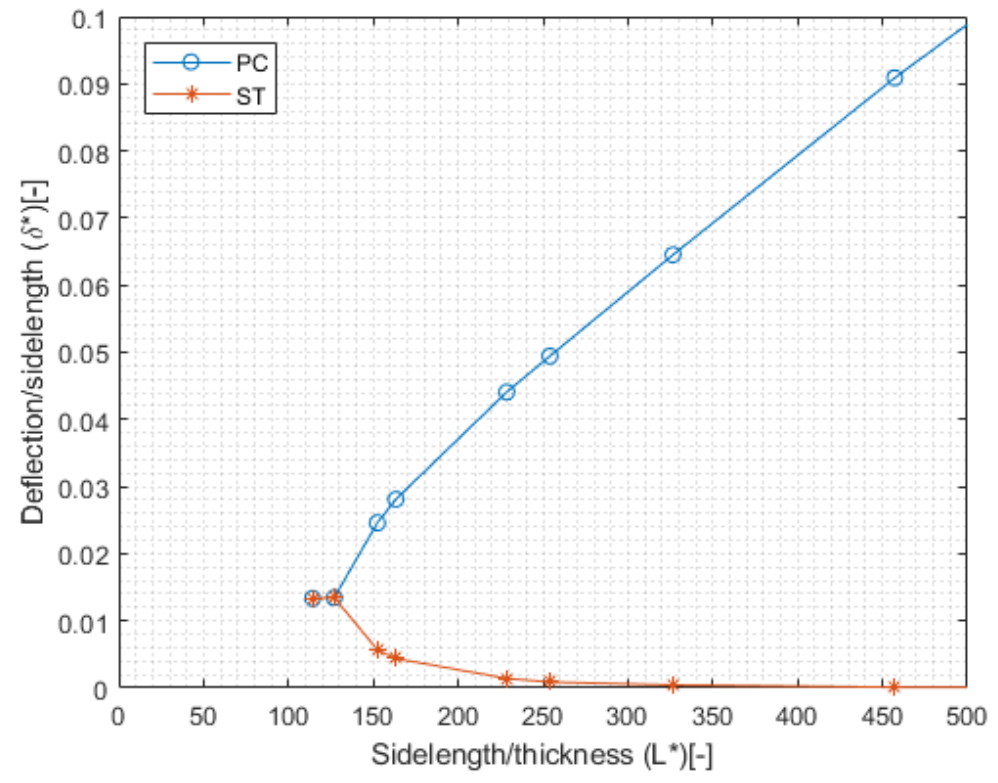

Figure 4.5: Rayleigh-Ritz Bifurcation of Deflections for DA409.

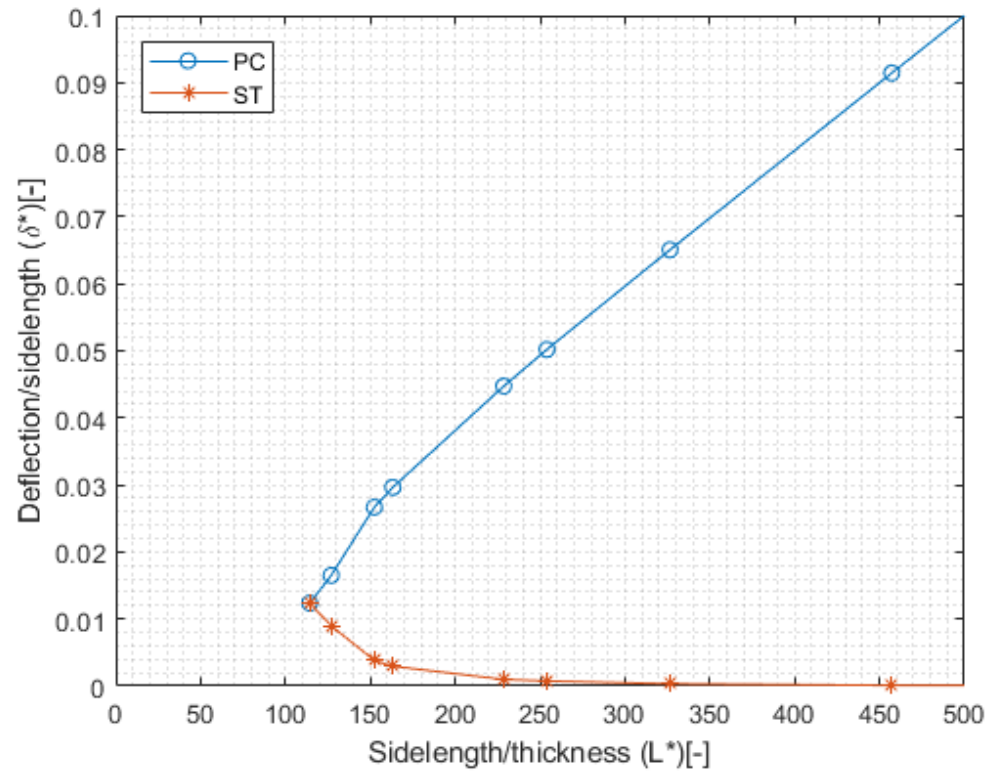

Figure 4.6: Rayleigh-Ritz Bifurcation of Deflections for TR50S. 
to generate maximum absolute deflections relative to both principle material axes. These peak deflections were then used to create bifurcation plots for determination of critical points, as seen for DA409 and TR50S in Figures 4.5-4.6, respectively, where the peak deflections relative to the x-axis were used.

Since sidelength was held constant for both length and width of laminates, the nondimensional aspect ratio sidelength-to-thickness was used in combination with the nondimensionalized quantity deflection-to-sidelength. The formulas used are:

$$
\begin{aligned}
L^{*} & =L / T=\frac{L}{n t} \\
w^{*} & =w_{\max } / L
\end{aligned}
$$

where $L$ is the sidelength, $T$ is the total laminate thickness as a function of number of plies $(n), t$ is the lamina thickness, and $w_{\max }$ is the peak deflection. From Figure 4.5, the DA409 critical point is found to be at $L^{*}=125.9$ which corresponds to 36 plies. Similarly from Figure 4.6 , the TR50S critical point is $L^{*}=114.3$, corresponding to 40 plies. These critical points are visual indicators that bistability is lost. The difference in $L^{*}$ values for critical points is due to TR50S's higher $E_{1}$ stiffness and $G_{12}$ shear stiffness moduli.

Bifurcation of curvature plots were also created from the values of $w_{10}$ and $w_{01}$ for both materials and are shown in Figures 4.7-4.8. Curvature was nondimensionalized using the formula found in equation 4.3. The curvatures used are constant $P_{1}$ functions over the domain since $w_{10}$ and $w_{01}$ are constant in value. These curvatures also used the referenced the $\mathrm{x}$-axis as their datum.

$$
\kappa^{*}=(\kappa)(L)
$$

In Figures 4.7-4.8, the bifurcation of curvature plots had critical points at the same $L^{*}$ values as was indicated in the bifurcation of deflection plots. The suggests that the Hyer extension is can estimate the loss of bistability through either deflection or curvature based bifurcation. The plots also suggest show that as laminate thickness increases, the curvature decreases. Additionally, there exists one axis at the peak of the curvature that remains relatively constant in value until the 


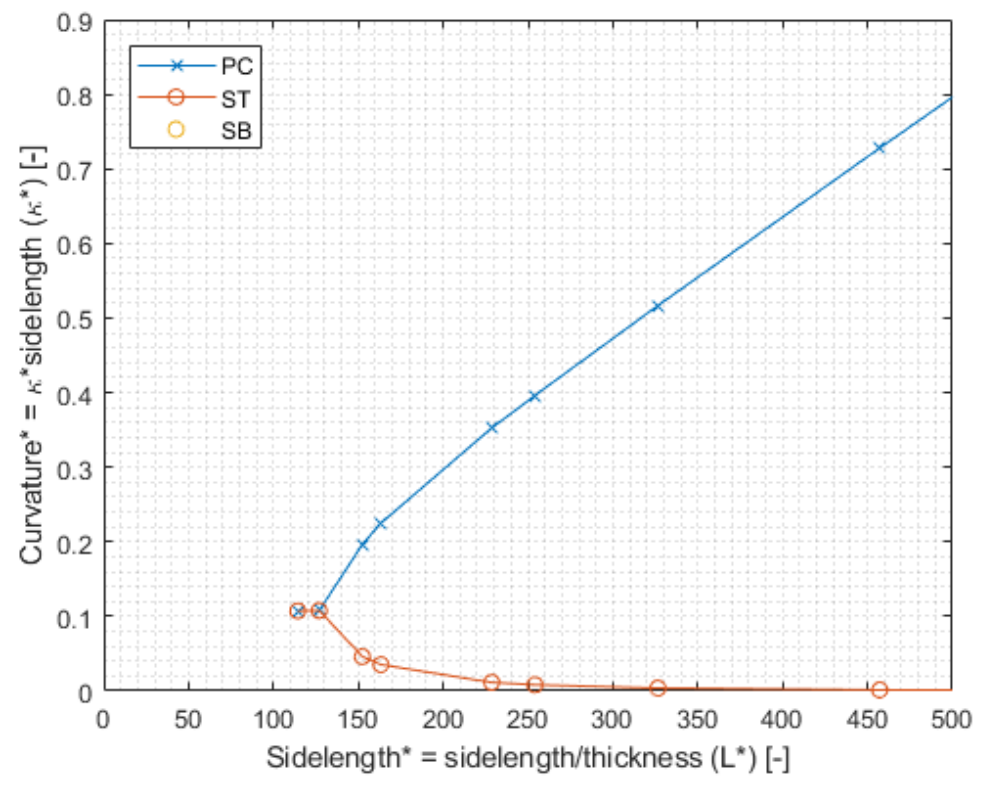

Figure 4.7: Rayleigh-Ritz Bifurcation of Curvature for DA409.

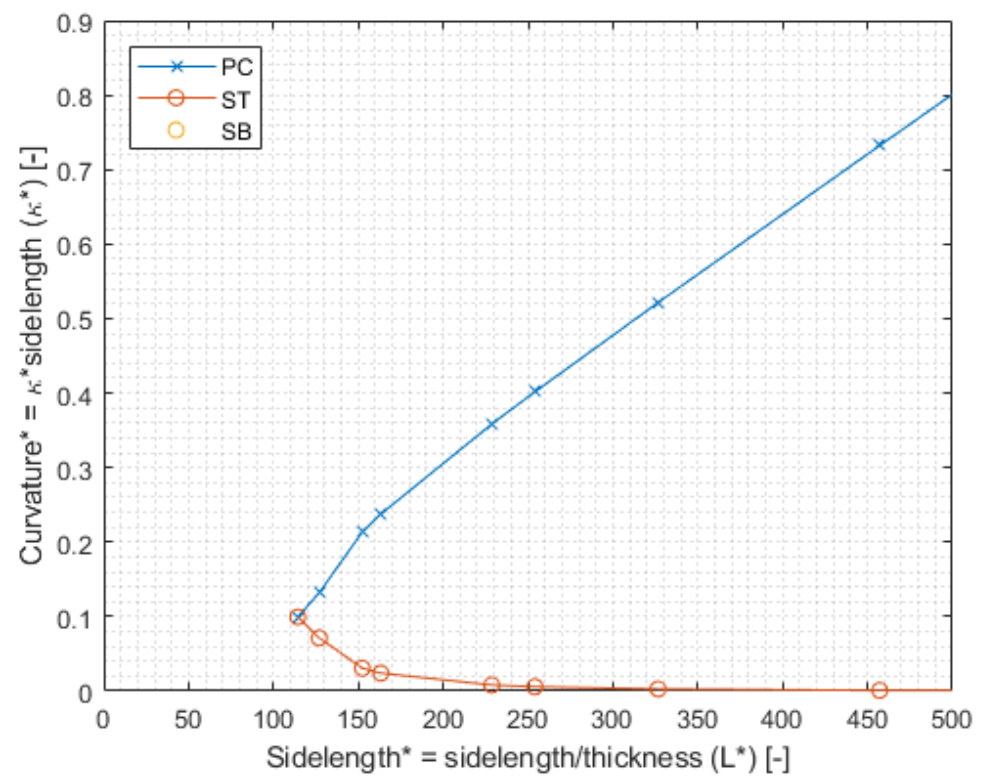

Figure 4.8: Rayleigh-Ritz Bifurcation of Curvature for TR50S. 
thickness is increased substantially upon reaching the critical points.

\subsubsection{Finite Element Model Cure Shape Predictions}

The second approach undertaken to predict the cure shape of asymmetric bistable laminates was the use finite element analysis software. For ease of inputting parameters into Abaqus, millimeter/Newton/second/milliJoule were used. As Abaqus does not keep track of units, to remain consistent, Megapascals were used when inputting moduli from Table 3.1. Data was extracted through the use of Field Output requests and History Output requests (native to Abaqus) and exported to Microsoft Excel files which were analyzed in MATLAB.

Mesh refinement was done systemically until results for FEA simulations converged. The mesh was iteratively decreased in size by $50 \%$ until convergence occurred. For all laminates, this resulted in approximately $1600 \mathrm{~S} 4 \mathrm{R}$ elements with approximate global seed sizes of $23 \mathrm{~mm}$. If the mesh was decreased further, no greater accuracy of data was observed yet time needed for completion of the simulation was increased substantially.

The results of FEA gave paraboloid curved laminates after the completion of the cooling step calculation. As the thickness increased, the magnitude of the curvature decreased on the $\mathrm{x}$ axis while the magnitude of curvature increased slightly on the y-axis. This relationship shows two things. The first is that there exists edge prediction yielded by FEA; and second, as the laminates thickness increases, the more likely that an unstable saddle shape will occur. The post-cure stable configuration for a 30-ply DA409 sample are shown in Figure 4.9.

Bifurcation plots generated based on deflection data of the centroid was determined. The peak deflection was determined from a $P_{4}$ polynomial best fit to the 11 displaced nodes used, shown for DA409 in Table 4.2 and for TR50S in Table 4.3. For each laminate, the $P_{4}$ bestfit equation was different and it is definitely dependent on thickness. However, the physical meaning of the coefficients is unknown.

Nondimensionalized peak deflections were plotted against $L^{*}$ and shown in Figure 4.10 for DA409 and Figure 4.11 for TR50S. Note that the deflections in Figures 4.9 and 4.10 do not converge to a bifurcation point when the value of $L^{*}<100$. This is indicative that bistability was not found when up to 60-ply thicknesses were examined. However, at this point even in spite of the absence of critical points, the magnitude of principle deflections only differed by one order of magnitude ( $L_{x} *=0.0377$ and $\left.L_{y} *=0.0039\right)$ where the difference may not be large enough to be evidence of 


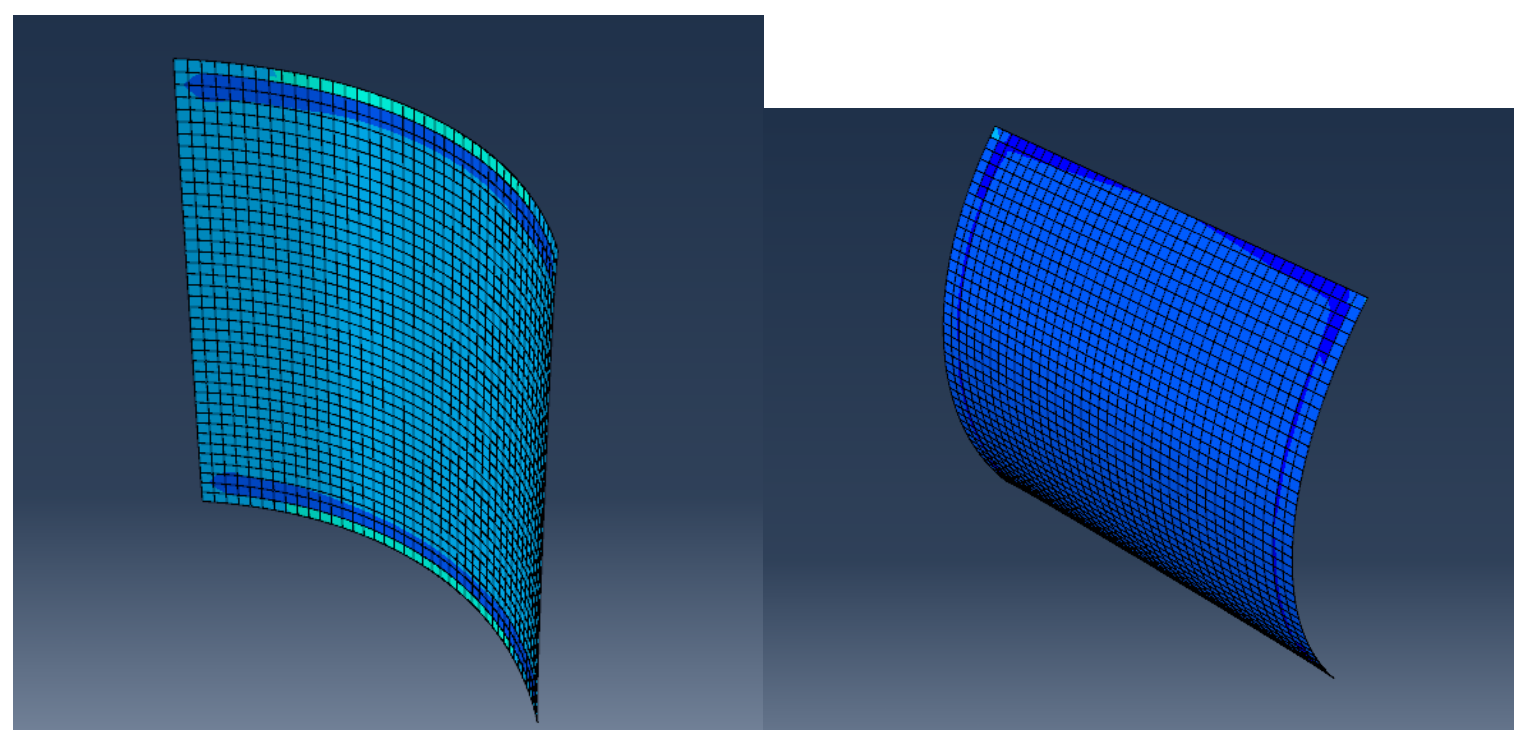

(a) Config. A.

(b) Config. B

Figure 4.9: Post-Cure Visualizations of Stable Shapes for 14-Ply DA409.

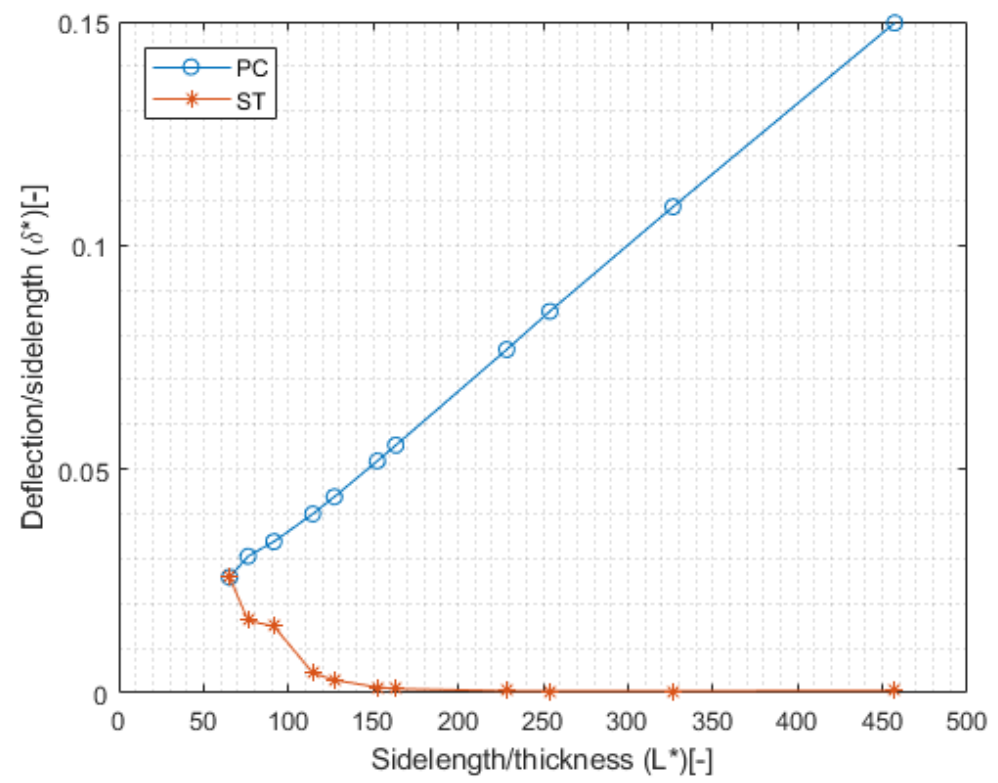

Figure 4.10: Bifurcation of Deflection for DA409 Generated from FEA. 


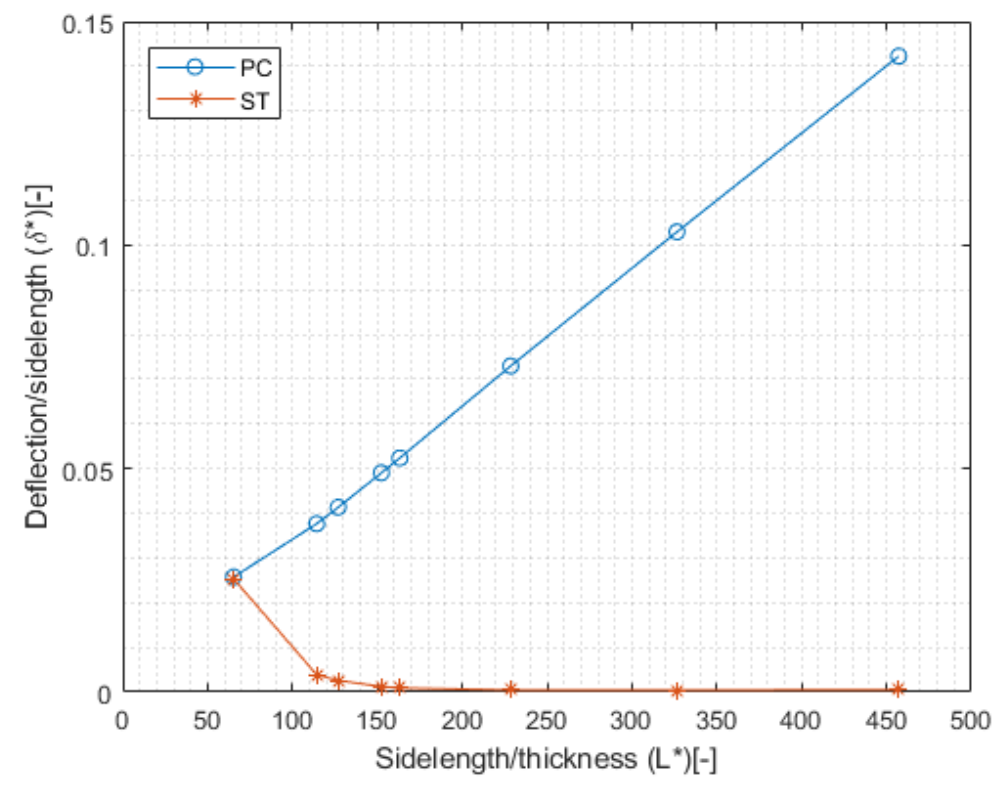

Figure 4.11: Bifurcation of Deflection for TR50S Generated from FEA.

\begin{tabular}{|c|c|c|c|c|c|}
\hline n-plies & $x^{4}$ & $x^{3}$ & $x^{2}$ & $x$ & $x^{0}$ \\
\hline \hline $\mathbf{1 0}$ & $1.59 \mathrm{E}-09$ & $-3.56 \mathrm{E}-08$ & $-1.88 \mathrm{E}-06$ & $2.16 \mathrm{E}-05$ & $8.63 \mathrm{E}-04$ \\
$\mathbf{1 4}$ & $3.98 \mathrm{E}-10$ & $-1.70 \mathrm{E}-08$ & $8.59 \mathrm{Ee}-07$ & $1.56 \mathrm{E}-05$ & $6.25 \mathrm{E}-04$ \\
$\mathbf{1 8}$ & $1.66 \mathrm{E}-10$ & $-9.14 \mathrm{E}-09$ & $-5.51 \mathrm{E}-07$ & $1.21 \mathrm{E}-05$ & $4.92 \mathrm{E}-04$ \\
$\mathbf{2 0}$ & $1.17 \mathrm{E}-10$ & $-6.88 \mathrm{E}-09$ & $-4.67 \mathrm{E}-07$ & $1.07 \mathrm{E}-05$ & $4.44 \mathrm{E}-04$ \\
$\mathbf{2 8}$ & $3.96 \mathrm{E}-11$ & $-2.66 \mathrm{E}-09$ & $-2.92 \mathrm{E}-07$ & $7.69 \mathrm{E}-06$ & $3.20 \mathrm{E}-04$ \\
$\mathbf{3 0}$ & $3.10 \mathrm{E}-11$ & $-2.18 \mathrm{E}-09$ & $-2.64 \mathrm{E}-07$ & $7.13 \mathrm{E}-06$ & $2.97 \mathrm{E}-04$ \\
$\mathbf{3 6}$ & $1.54 \mathrm{E}-11$ & $-1.24 \mathrm{E}-09$ & $-2.00 \mathrm{E}-07$ & $5.84 \mathrm{E}-06$ & $2.46 \mathrm{E}-04$ \\
\hline
\end{tabular}

Table 4.2: DA409: Coefficients for $P_{4}$ Polynomials Describing Deflection Shape Generated from FEA.

\begin{tabular}{|c|c|c|c|c|c|}
\hline - plies & $x^{4}$ & $x^{3}$ & $x^{2}$ & $x$ & $x^{0}$ \\
\hline \hline $\mathbf{1 0}$ & $1.33 \mathrm{E}-09$ & $-3.17 \mathrm{E}-08$ & $-1.71 \mathrm{E}-06$ & $2.05 \mathrm{E}-05$ & $8.22 \mathrm{E}-04$ \\
$\mathbf{1 4}$ & $3.55 \mathrm{E}-10$ & $-1.49 \mathrm{E}-08$ & $-8.10 \mathrm{E}-07$ & $1.47 \mathrm{E}-05$ & $5.94 \mathrm{E}-04$ \\
$\mathbf{2 0}$ & $1.17 \mathrm{E}-10$ & $-5.94 \mathrm{E}-09$ & $-4.72 \mathrm{E}-07$ & $1.03 \mathrm{E}-05$ & $4.26 \mathrm{E}-04$ \\
$\mathbf{2 8}$ & $3.93 \mathrm{E}-11$ & $-2.28 \mathrm{E}-09$ & $-2.97 \mathrm{E}-07$ & $7.28 \mathrm{E}-06$ & $3.04 \mathrm{E}-04$ \\
$\mathbf{3 0}$ & $3.09 \mathrm{E}-11$ & $-1.86 \mathrm{E}-09$ & $-2.69 \mathrm{E}-07$ & $6.78 \mathrm{E}-06$ & $2.84 \mathrm{E}-04$ \\
$\mathbf{3 6}$ & $1.5240 \mathrm{e}-11$ & $-1.0795 \mathrm{e}-09$ & $-2.0498 \mathrm{e}-07$ & $5.58 \mathrm{E}-06$ & $2.33 \mathrm{E}-04$ \\
\hline
\end{tabular}

Table 4.3: TR50S: Coefficients for $P_{4}$ Polynomials Describing Deflection Shape Generated from FEA. 


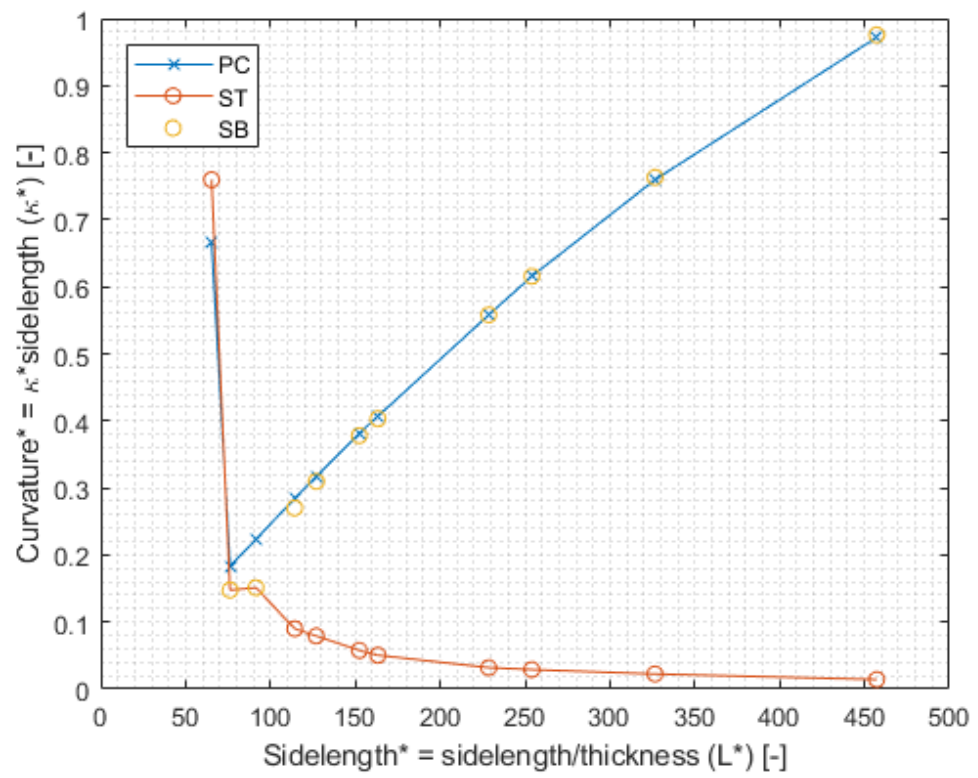

Figure 4.12: Bifurcation of Curvature for DA409 Generated from FEA.

two stable equilibrium configurations.

Moreover, bifurcation of curvature plots were created. The curvature plots are related to the deflection plots by way of Equation 3.2. A detail of importance is that Equation 3.2 may cause sensitivity in the actual calculation of curvatures since it utilizes second-order derivatives. Bifurcation of curvature for DA409 and TR50S are shown in Figures 4.12 and 4.13, respectively.

Similarly to the bifurcation of deflection plots, Figures 4.12-4.13 indicate sidelength-tothickness ratios less than 125 suggesting that upwards of 60 plies are needed to cause loss of bistability. This data contradicts heavily with Rayleigh-Ritz by almost doubling the ply count. Of interest, is that the FEA still maintains polynomial prediction of curvature over the domain, although Figures 4.12-4.13 only show mean curvature. The higher than trend data for curvatures, at $L^{*}=67$ is indicative of saddle shape.

Thus, due to the sidelength-to-thickness ratios below $L_{\text {critical }}^{*}$, the data was indicative of the idea that FEA bifurcation plots were not reliable for prediciting bistability loss. Investigation of energy landscapes were done to determine if there was any other evidence suggesting a different critical point for bistability loss. The energy landscape is the potential strain energy plotted against time or displacement, where the energy is gathered from an Abaqus Field Output Request. In this 


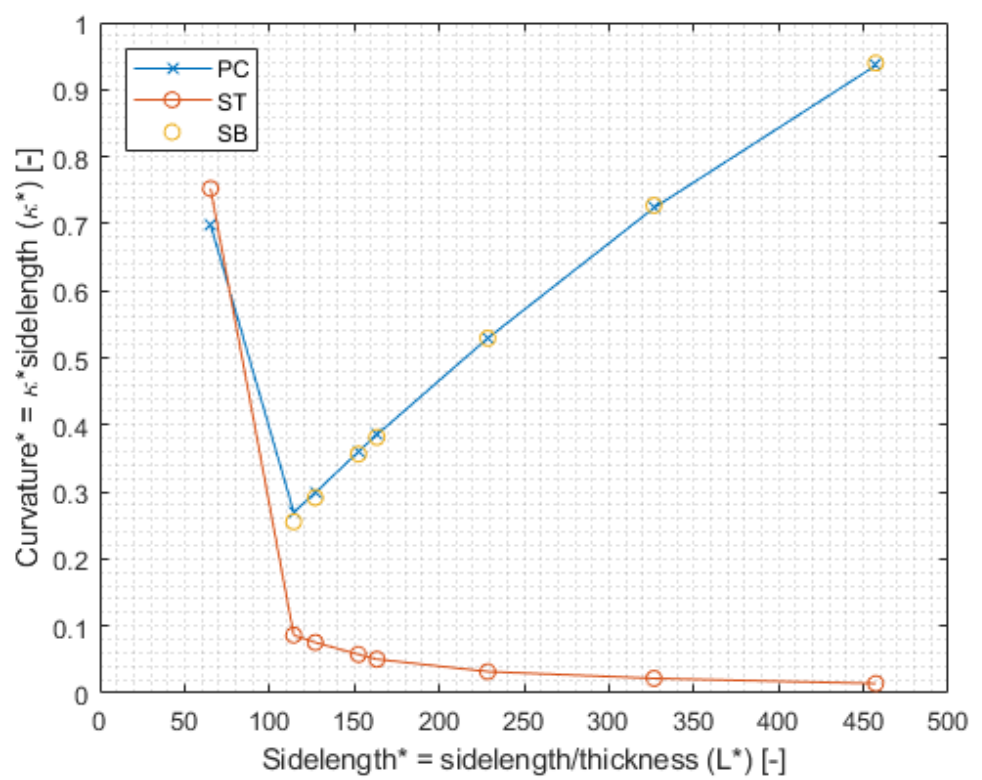

Figure 4.13: Bifurcation of Curvature for TR50S Generated from FEA.

study, time is used to better relate inflection points to STEP events within Abaqus. For $t=1 \mathrm{~s}$ is when the end of the post-cure step is done. For $t=3 \mathrm{~s}$ or $t=5 \mathrm{~s}$ is the end of a loading step, when the laminate should have settled into a natural position. Figure 4.14 shows the TR50S energy landscapes for selected thicknesses, as indicated by the color-coded legend. Figure 4.14 does not indicate any clear trends of all laminates except that they follow the same loading and unloading procedures.

Comparisons between different laminate thicknesses could only be done if energy was nondimensionalized so that the different energy landscapes would collapse together. This was done through Equation 4.4:

$$
E^{*}=\frac{E}{L^{2} T L E_{1}}
$$

where $E$ is strain energy of the laminate determined from a Field Output Request, $L$ is sidelength, $T$ is total thickness as function of the number of lamina (n), and $E_{1}$ is the lamina principle elastic modulus. 


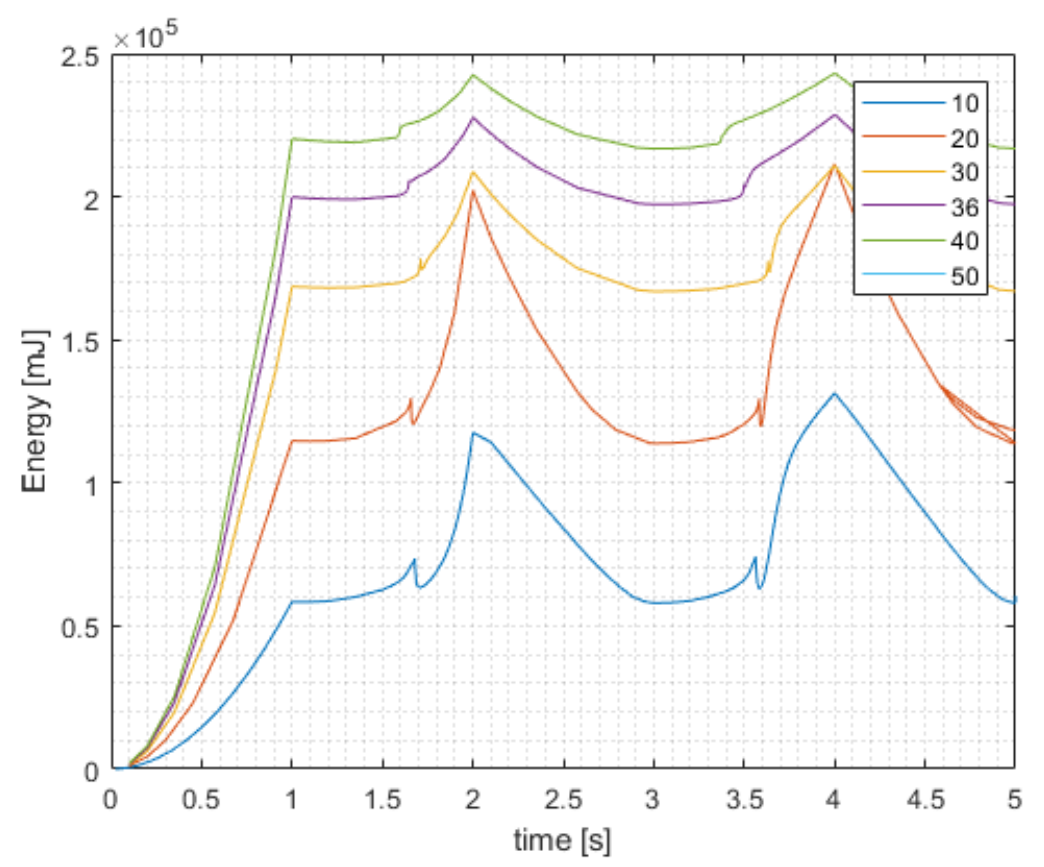

Figure 4.14: Selected Energy Landscapes of TR50S.

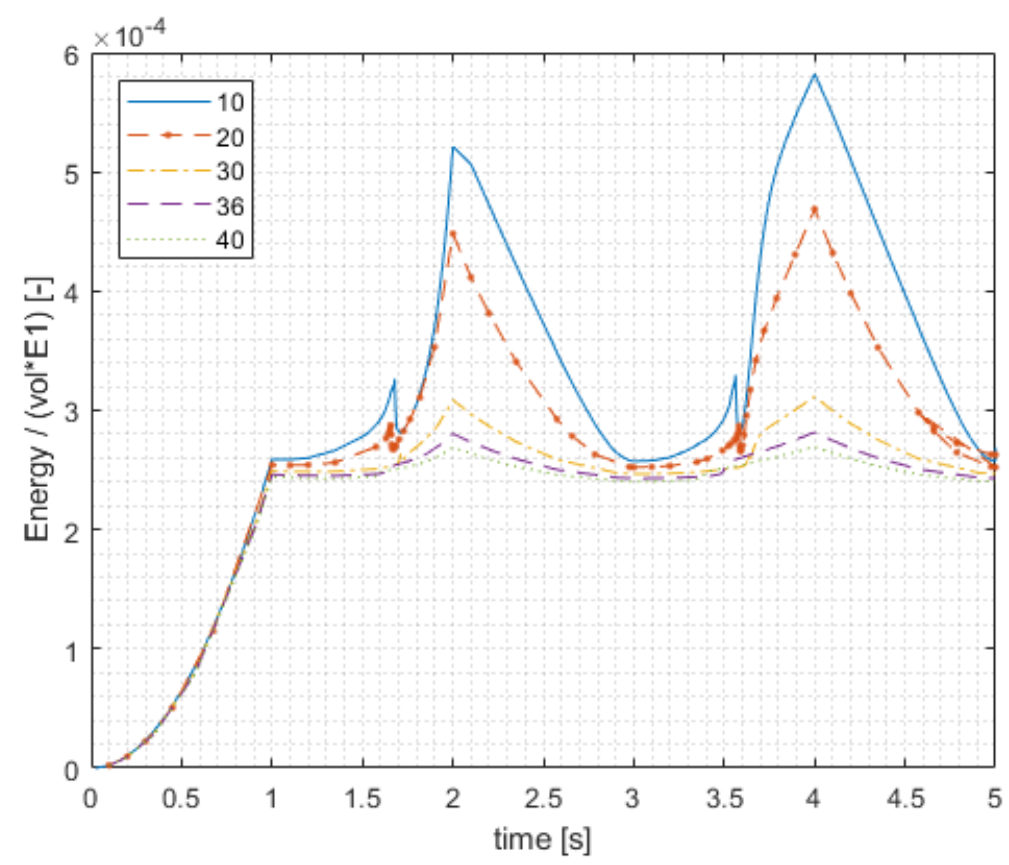

Figure 4.15: Selected Nondimensionalized TR50S Energy Landscapes. 


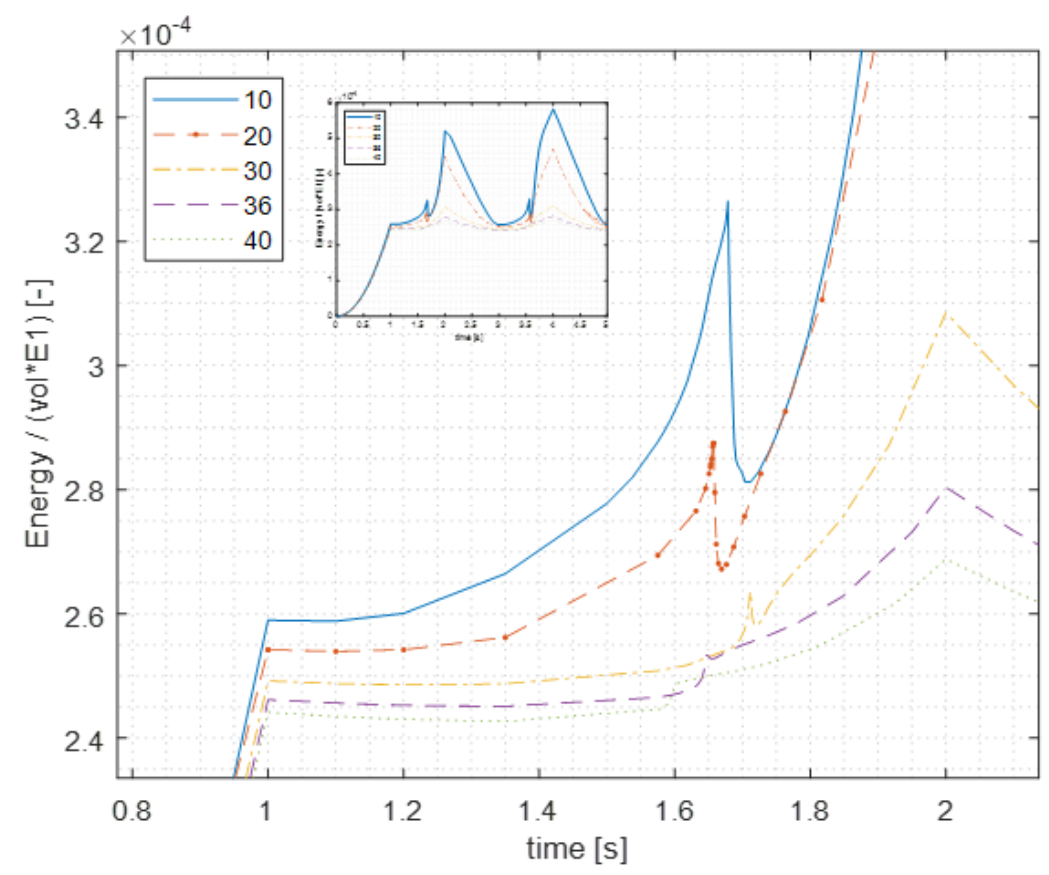

Figure 4.16: Selected Nondimensionalized TR50S Energy Landscapes from $0.8<t<2$. Inset: Figure 4.15.

The same TR50S energy landscapes, nondimensionalized, are shown in Figure 4.15 and Figure 4.16 shows the energy landscapes from $0.8 s<t<2.2 \mathrm{~s}$. In Figure 4.16 , the energy curves have collapsed together but start to separate after the post-cure load STEP of the simulation (4.15 Inset). A point of interest is the large delta in energy with multiple inflection points at approximately $t=1.7 \mathrm{~s}$ in Figure 4.16. As number of plies increases, the point eventually disappears. This point occurs as a result of the snapthrough loading scenario as the strain energy will decrease suddenly. Absence of this point indicates no snapthrough occured even though applied load is increased; rather, it indicates an elastic displacement. Hence, for laminates whose plots that exhibit small or no snapthrough point are indicative of a laminate with only unstable configurations.

Perhaps this is more indicative of loss of bistability for FEA than bifurcation plots relying on curvature. However, adjusting thickness between a pre-cure thickness and post-cure thickness in FEA studies during calibration, has shown tremendous effects on previously found bifurcation points suggesting that bistability for the FEA model is dependent on at least thickness per ply. This interpretation falls more in line with the 152 length-to-thickness ratio determined by [9]. However, this interpretation also brings up the idea of a bifurcation critical point existing as a region on the 


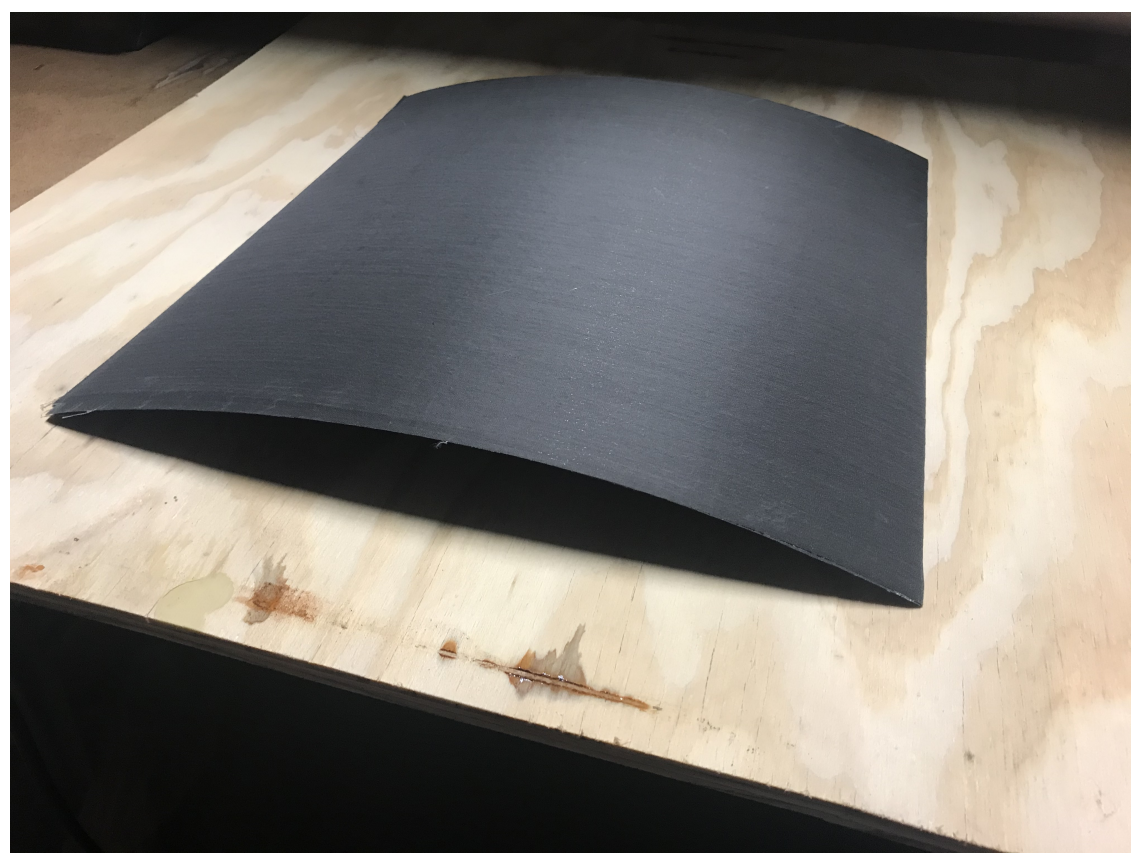

Figure 4.17: Stable Configuration A for a 6-Ply 325mm x 325mm Sample.

curvature-sidelength figures.

\subsubsection{Shape Comparisons Between Models and Experiments}

Experiments were carried out to verify trends and results obtained from both modeling approaches. The Rayleigh-Ritz method utilizes a plane stress assumption while FEA maintains a 3-D shell assumption. These assumptions are delimiting factors in determining effects and usefulness of these modeling approaches. Experimental validation helps decipher any discrepancies. Figures 4.17-4.18 show the two stable equilibrium positions for a 12-ply DA409 laminate.

Experimental deflections were measured for laminates of DA409 from 10 plies to 28 plies in thickness. At 28 plies and thicker, DA409 laminates did not show bistability. Prior to this thickness, samples were all without question bistable. However, measurements on the deflections were still obtained and can be seen in Figure 4.19 with deflections from the Hyer extension and FEA. Observations of TR50S were mixed concerning the presence of bistability. Samples of TR50S were made from 10 plies to 36 plies with bistability observed in 30 and 36 ply samples. Deflection measurements were taken for these two samples 3-point approximations but these samples were donated to SPAWAR (now known as NIWC Atlantic) and no further measurements were obtained. 


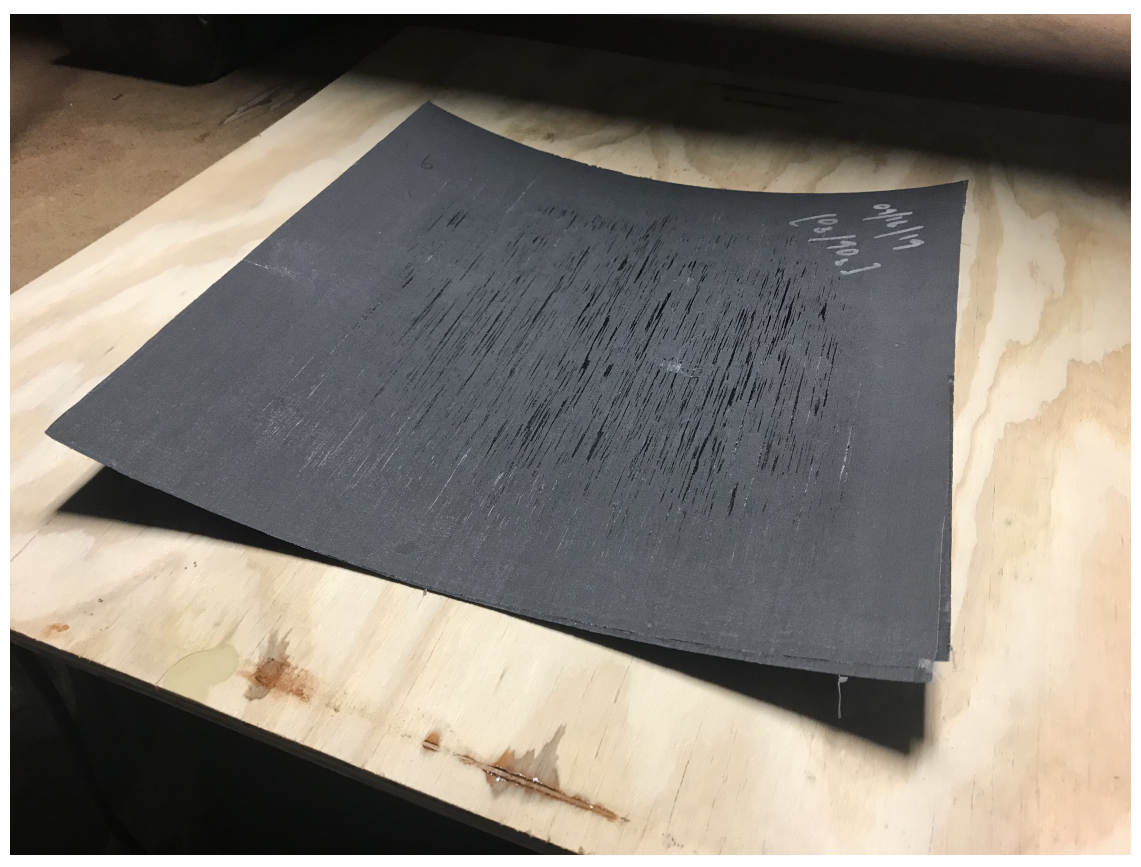

Figure 4.18: Stable Configuration B for a 6-Ply 325mm x 325mm Sample.

An attempt was made to recreate these samples for further study but upon fabricating two 28-ply TR50S samples, neither exhibited bistability. This could be due to subtle differences in prepreg unitape material since a new batch was being used from a different manufacturer under the same specifications.

Right away, it is easy to tell from Figures 4.19-4.20, that the critical points determined from Rayleigh-Ritz and experiments for DA409 and TR50S are similar. Furthermore, it is obvious that FEA predictions when compared to experiments are contradicting in nature. This could be attributed to some physical aspect anecdotally observed in experiments that is not being captured in FEA. The experimental critical point for DA409 experiments is $L^{*}=163$ according to deflection and curvature measurements. However, it was observed that samples that were 28 plies or more in thickness were not bistable, agreeing with the critical $L^{*}=152$. Abnormalities such as the increase in deflection for experiments at $L^{*}=127$ in Figure 4.20 is likely due to the fact that the prepreg material used for this laminate came from a different batch and manufacturer. Similarly, in Figure 4.19 the experimental post-cure deflection at $L^{*}=229$ (gold arrow) is likely due to that specific laminate having a high number of defects in addition to coming form a different batch.

When considering DA409 curvature (Figure 4.21), the critical point occurs at $L^{*}=127$ for 


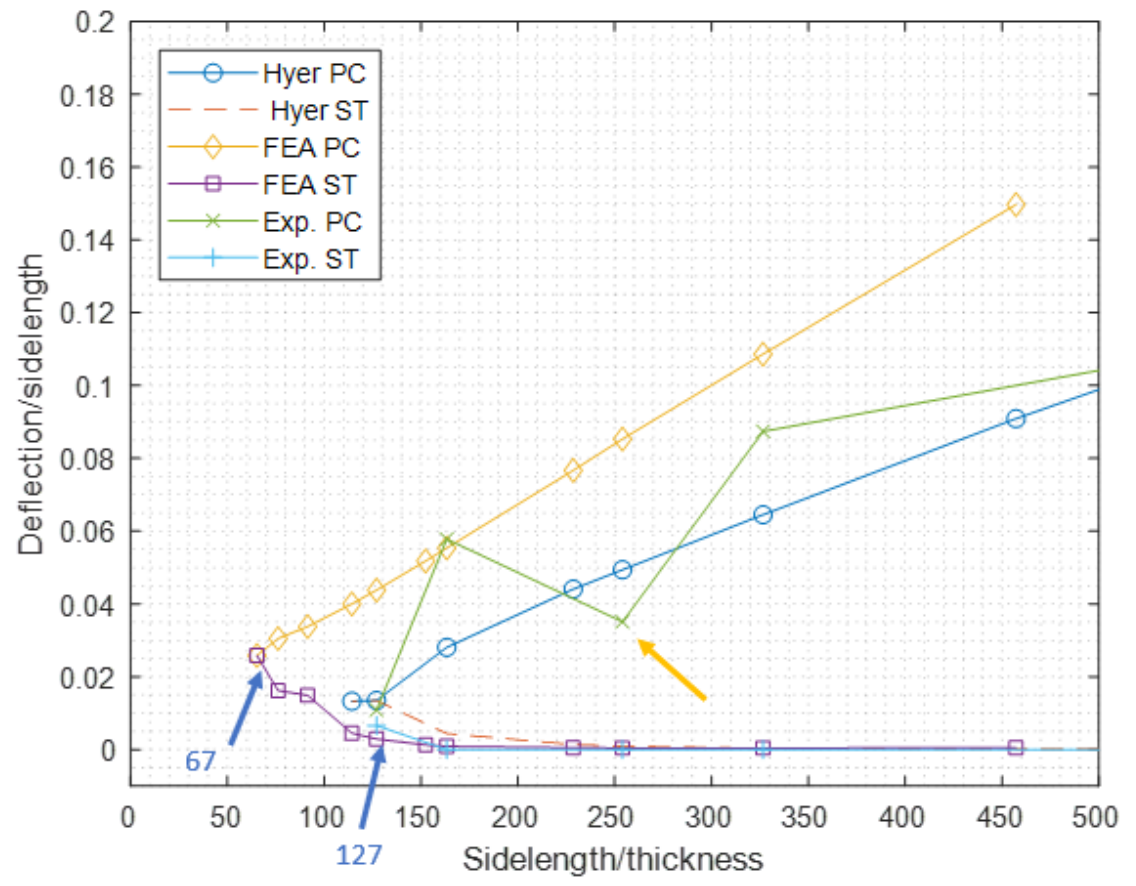

Figure 4.19: Nondimensional DA409 Experimental Deflection Compared to Rayleigh-Ritz and FEA.

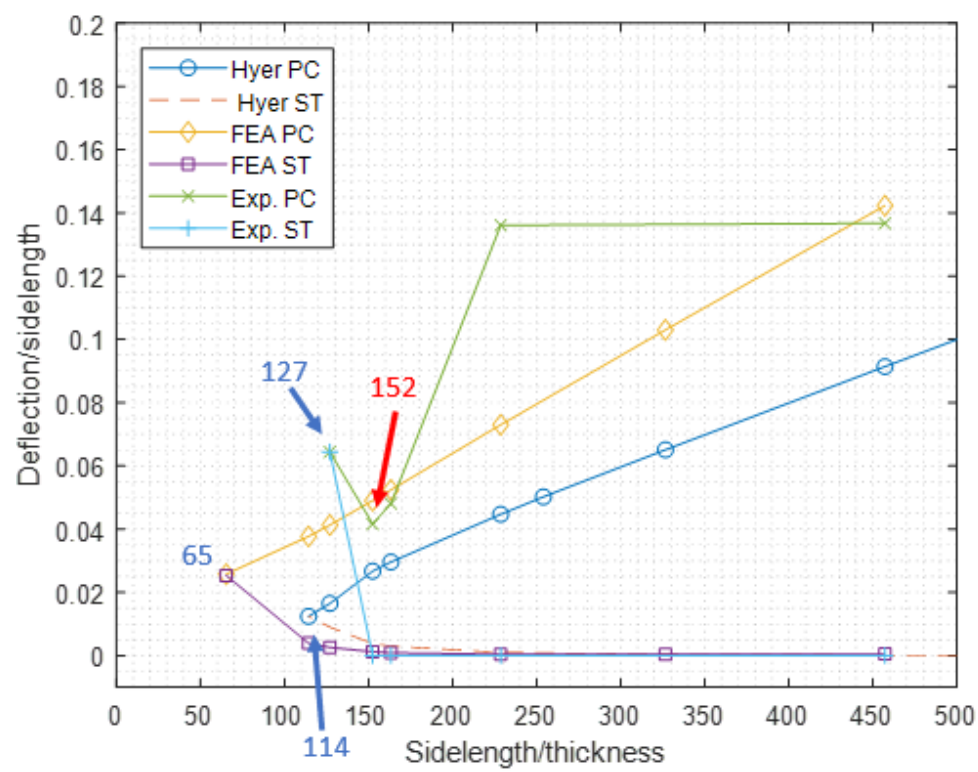

Figure 4.20: Nondimensional TR50S Experimental Deflection Compared to Rayleigh-Ritz and FEA. 


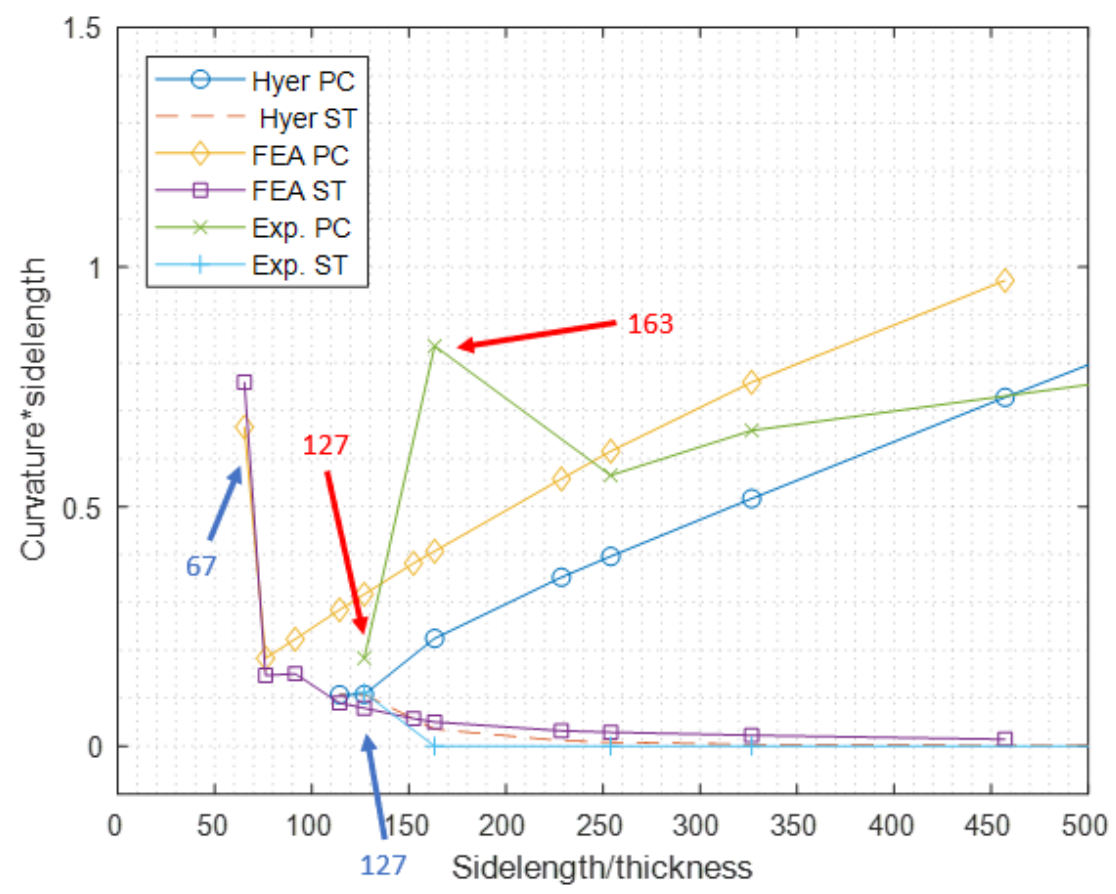

Figure 4.21: Nondimensional DA409 Curvature for Rayleigh-Ritz, FEA, and Experimentation.

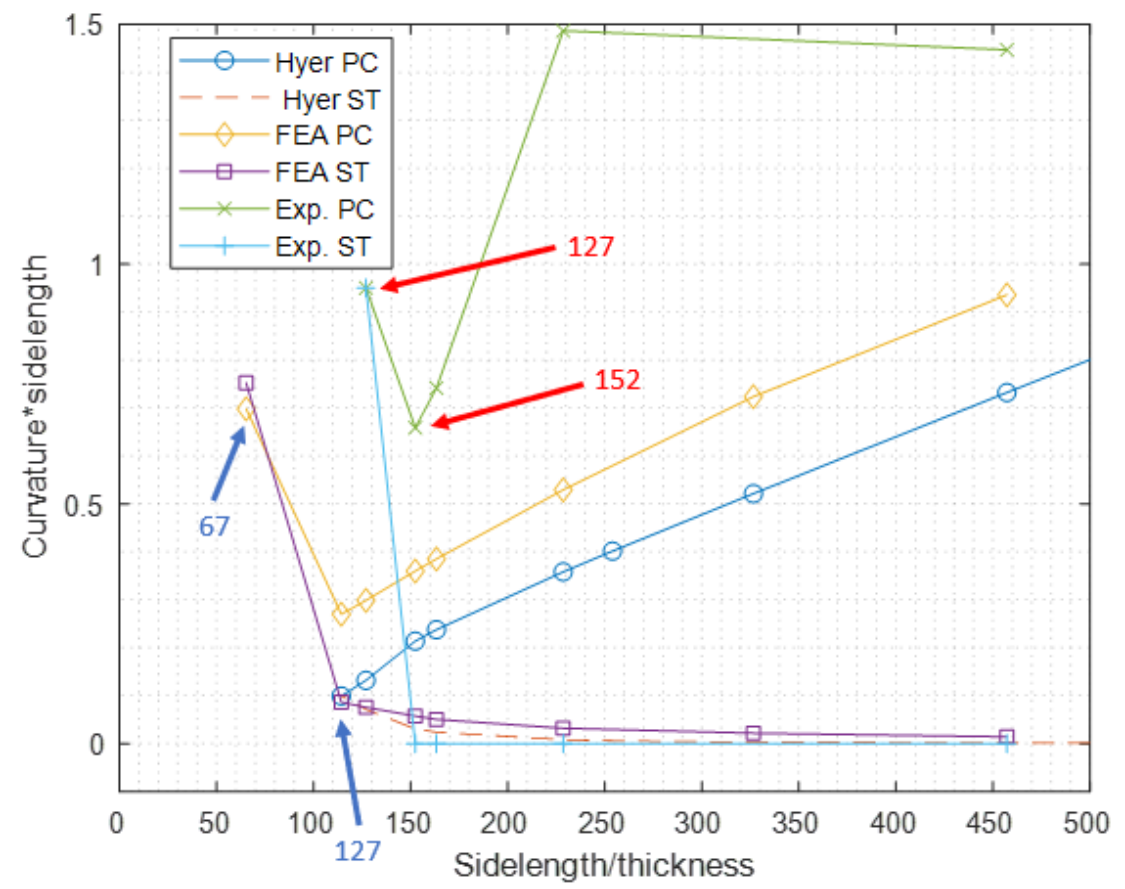

Figure 4.22: Nondimensional TR50S Curvature for Rayleigh-Ritz, FEA, and Experimentation. 


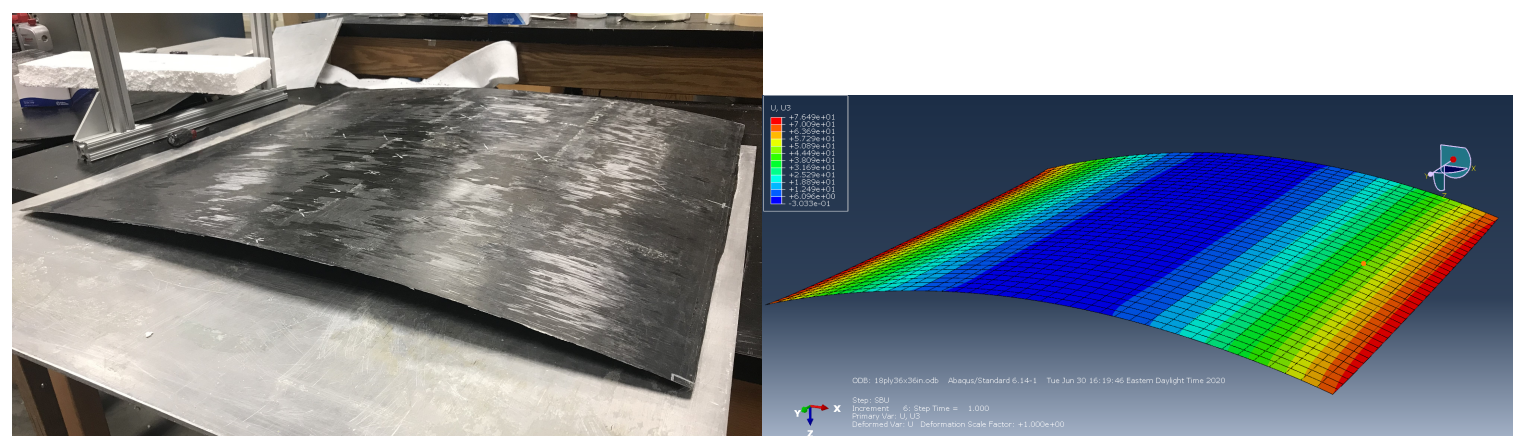

(a) Sample.

(b) FEA 18-ply.

Figure 4.23: DA409 18-Ply Cure-Shape Side-by-Side Visualization from FEA and Experiments.

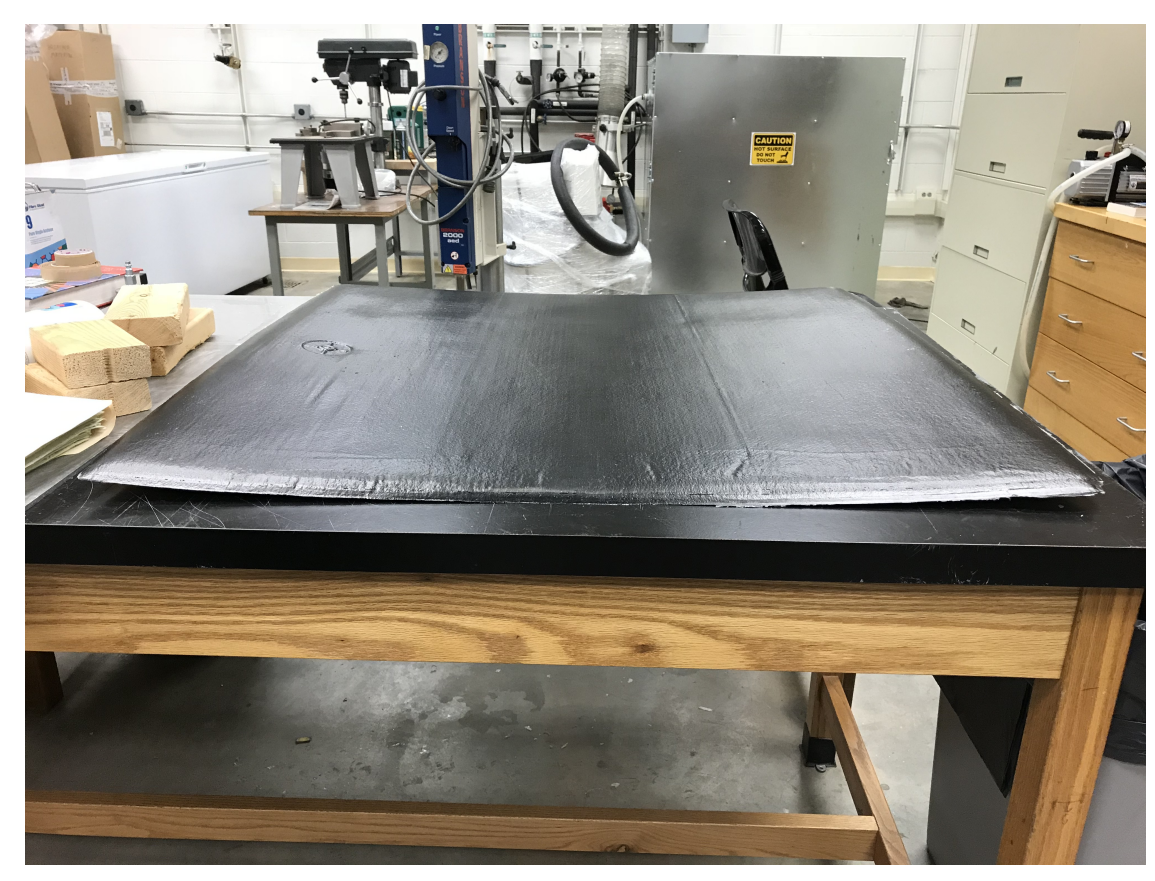

Figure 4.24: DA409 36-Ply Saddle Shape Unstable Sample. It was Observed that Axis Curvatures were not Equal in Magnitude. 
experiments, which agrees with results from Rayleigh-Ritz. For TR50S, the critical point is shown at $L^{*}=127$ and was also observed at $L^{*}=163$ corresponding to 36 and 28 plies, respectively. Of note, the saddle shape magnitudes for a 36-ply (AR of 127) laminate is substantially increased compared to the expected trend, as indicated by the -*- green plot-line. Similar trends are observed in the bifurcation of curvature predicted by FEA for a critical point of $L^{*}=67$. The outlier at $L^{*}=163$ from experiments in Figure 4.21 is probably because the material used for this sample was from a different batch. The likely overprediction of curvature in both Figures 4.21 and 4.22 could be due to the fact that a single curvature value calculated from 11 nodes across a singular principle axis is not very descriptive.

Analytical results agree with literature where stable solutions have the distinct possible of trifurcating into three possible solutions, two of which are stable even though Hyer did not discuss results for laminates with increased thickenss [4]. Trifurcated results existed for all laminate layups up to 34 plies implying that samples could be both bistable or saddle shaped. Experimental evidence suggests that due to variances and imperfections that these results need to be verified. Loss of bistability was clear in Hyer's analytical model because only one solution would exist for said thickness with equal magnitude of curvatures $\kappa_{x}=-\kappa_{y}$. It was not discussed but concavity of the laminates is easily obtained for Rayleigh-Ritz through the use of Gaussian curvature. Moreover, the Rayleigh-Ritz Hyer extension provided a better idea of bistability loss than FEA bifurcation plots. Thus, the plane stress assumption seemed reasonable in this case. However, the prediction of deflections from Rayleigh-Ritz plane stress when compared to experiments was on average $9 \%$ less for DA409 and 30\% less for TR50S. Curvatures from the Rayleigh-Ritz plane stress were found to be $23.1 \%$ less for DA409 and $71 \%$ less for TR50S when compared to experiments. Meanwhile, FEA showed that deflection predictions compared to experiments were on average $5.6 \%$ less for DA409 and $31 \%$ less for TR50S. Curvature predictions from FEA were on average $34 \%$ less for DA409 and $70 \%$ less for TR50S. The overprediction of curvatures is likely because curvature values were calculated from a bestfit line covering 11 nodes along a principle axis. Thus, the curvatures did not account for the other $1500+$ nodes that have varying magnitudes of curvature. If all 1600 nodes were taken into account, it is quite possible that FEA would have produced better results as far as curvature prediction is concerned.

In Figure 4.23, an 18-ply DA409 sample is shown next to the cure shape generated by FEA. Rayleigh-Ritz predicted the peak deflection as $45 \mathrm{~mm}$, FEA predicted $76 \mathrm{~mm}$ peak deflection, and 
experiments were measured to be $32 \mathrm{~mm}$ peak deflection. Figure 4.23 also shows the 18 -ply sample (a) with surface defects. Additionally, noticeable shape change had occurred with moisture exposure over time. Figure 4.24 shows the unstable cure shape of a 36-ply DA409 sample with noticeable saddle shape.

\subsection{Snapthrough behavior}

Snapthrough actuation studies were only done with finite element analysis and experiments. Details about how loads were determined follow as well discussion on observations.

\subsubsection{FEA Snapthrough Predictions}

Force-Displacement graphs were used to determine the actuation load necessary to cause snapthrough phenonema to occur in the bistable laminates. As can be seen in Figure 4.25 the force slowly increases almost linearly with displacement, then there occurs a large displacement for an almost constant value of force. This large displacements corresponds to the center of the laminate changing from one equilibrium position to the next [8]. Additionally, after this large displacement keep increasing due to the fact that larger loads than necessary for actuation were needed for modeling. In the Figure 4.25, for sample 14-ply TR50 laminate, the snapthrough load was found to be $63 \mathrm{~N}$.

Hence, snapthrough actuation load curves were created for DA409 and TR50S where the actuation load for snapthrough and snapback was plotted against the number of plies $(n)$ in Figure 4.26. The load curves were generated from FEA using a clamped loading condition. As would be expected, the thicker the laminates become, the more force is needed to actuation snapthrough. Yet, the actual magnitudes are relatively small. Daynes and Weaver stated that thermally induced residual stresses are the reason for this because thermal stress is small relative to other factors [3]. There also is an observable average $11 \%$ difference between snapthrough and snapback loads; for which, Hyer attributed to asymmetric cooling effects [4]. However, the FEA model was setup such that cooling was to occur uniformly. 


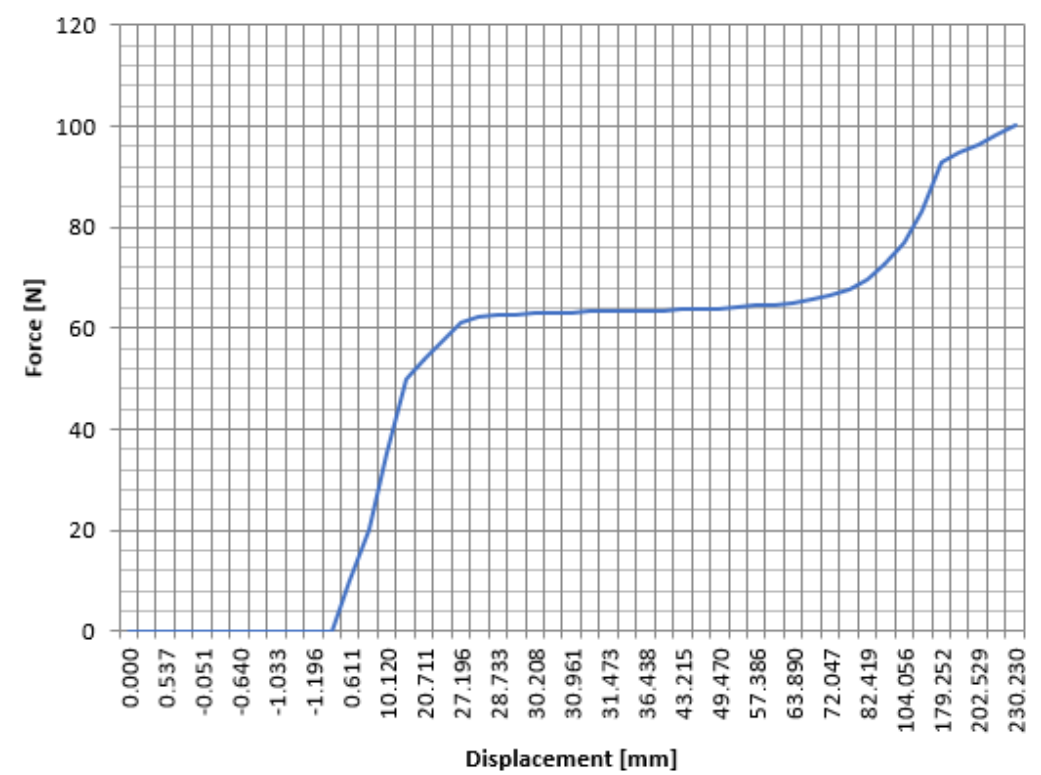

Figure 4.25: Force-Displacement of Centroid for 14-Ply TR50S Laminate.

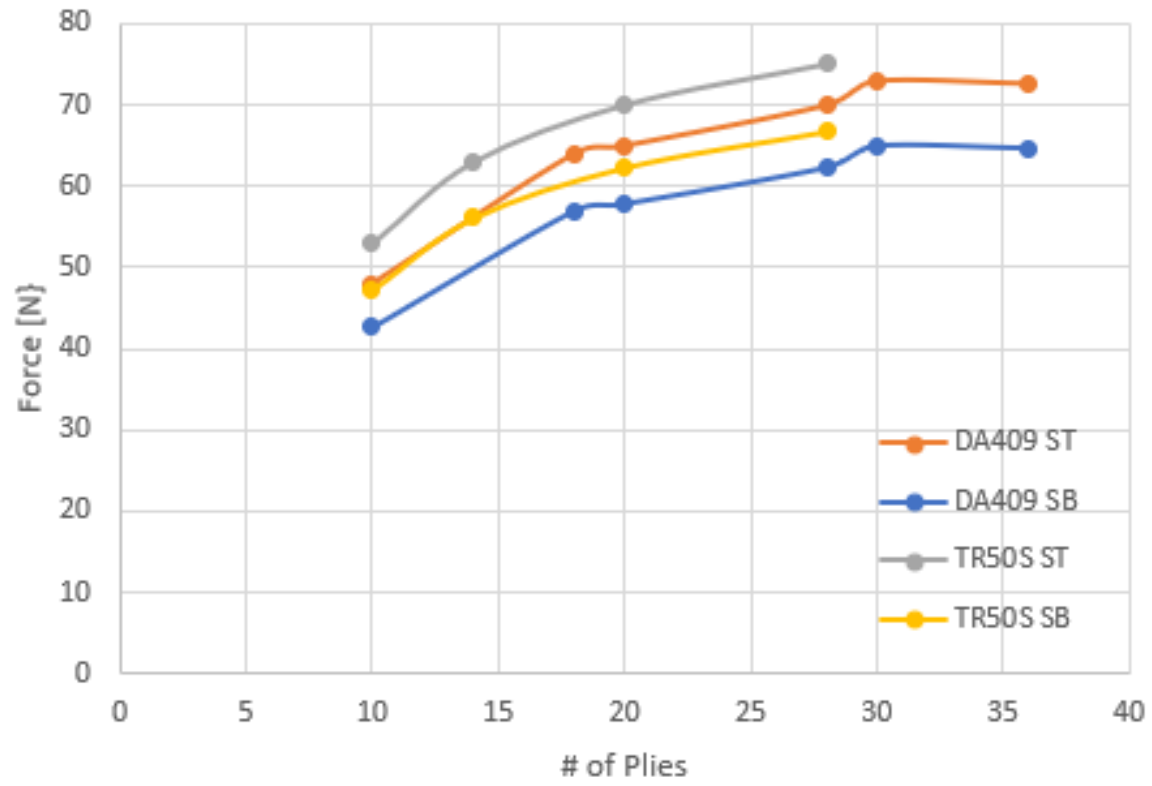

Figure 4.26: Snapthrough and Snapback Actuation Load Curves for DA409 and TR50S Predicted by Finite Element Analysis. 


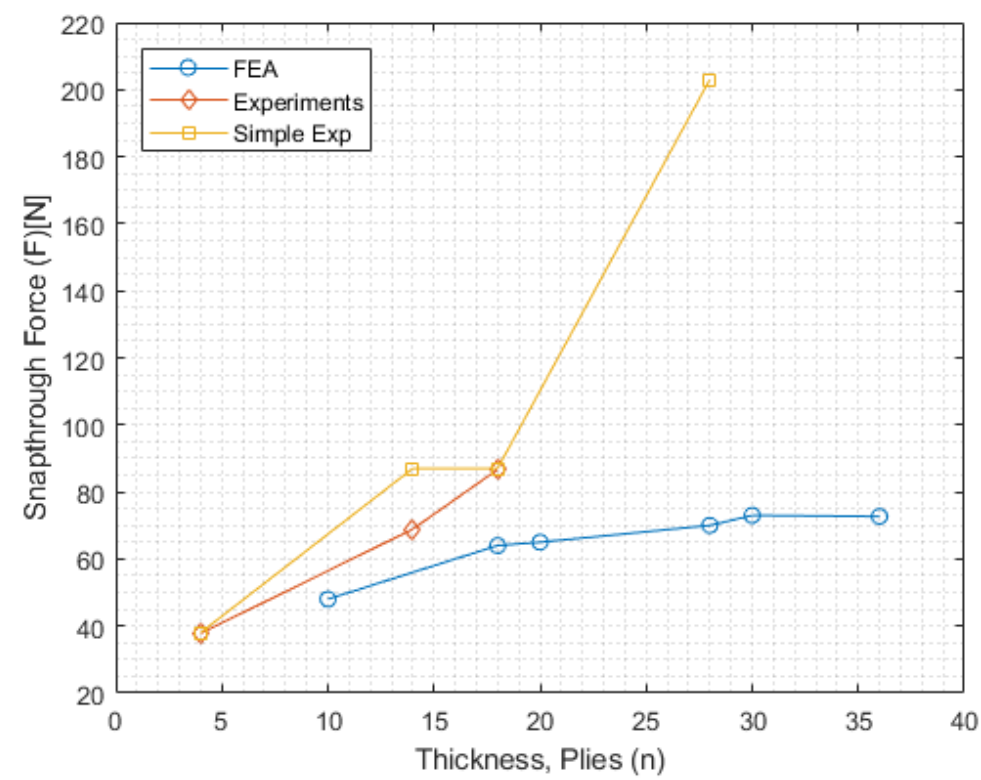

Figure 4.27: Snapthrough/Snapback Actuation Load Curve for DA409 as a Function of Number of Lamina (n).

\subsubsection{How FEA Predictions Compare to Experiments}

Experimental validation for snapthrough was performed to determine the accuracy of snapthrough load predictions. The snapthrough loads were pulled from Figure 4.25 and plotted with the measured actuation loads obtained from experiments.

As can be seen in Figure 4.27 for DA409, the direct comparison given for DA409 laminates ranging from 4-36 plies shows fairly linear increases in snapthrough loads. But, experiments show a steeper increase in load magnitude. Actuation load data was unobtainable for DA409 laminates greater than 18 plies (due to inability to create bistable samples) but between 10-18 plies, there was an average $30 \%$ difference in load magnitudes with the largest discrepancy being at 18 plies. This difference could be attributed to FEA's lack of capturing physical imperfections as well as the influence of gaps created with 0.314 wide DA409 unitape during fabrication. The simply supported experiments show a curve that is has similar behavior to FEA when thin but upon reaching 28-ply thickness, the actuation load increases dramatically to $203 \mathrm{~N}$. This is most likely since the laminate was on the edge of being unstable and acted more like a spring than a true bistable laminate.

Similarly for TR50S in Figure 4.28, the direct comparison shows FEA and experimental 


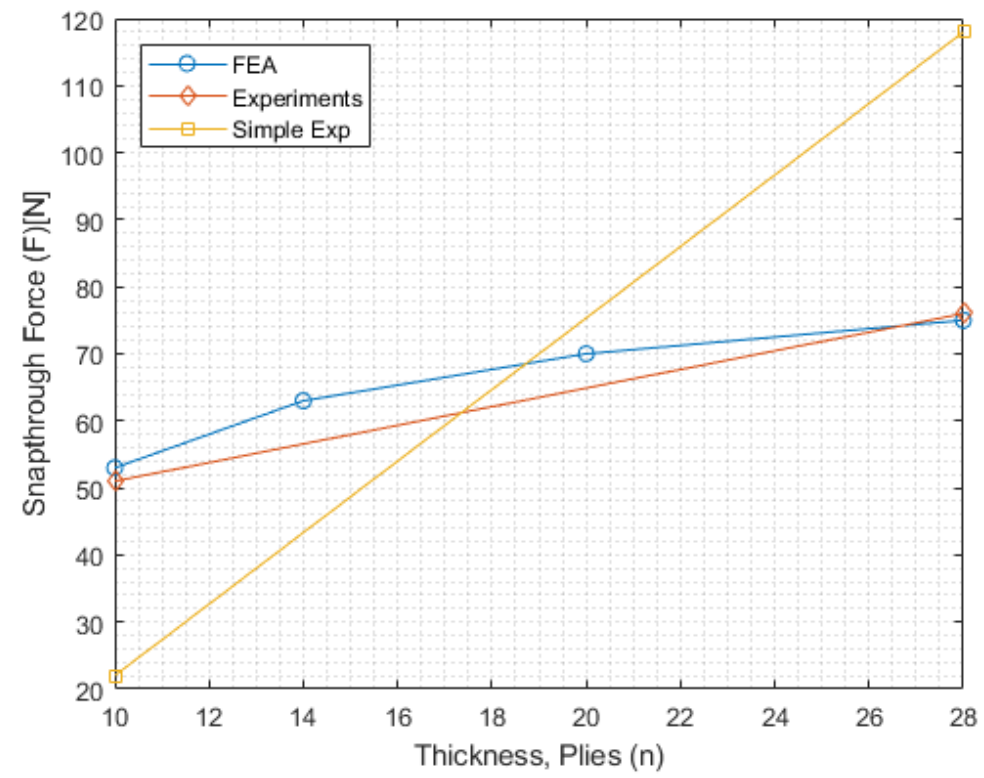

Figure 4.28: Snapthrough/Snapback Actuation Load Curve for TR50S as a Function of Number of Lamina (n).

predictions for snapthrough to be very similar in slope and magnitude with an exhibited average $2.5 \%$ difference. This is probably due to the fact that experimental samples were unavailable (donated to SPAWAR) and no longer available for measurement so that more points could be generated. Furthermore, the effects of moisture ingress also probably had a significant influence on experimental measurements. Simply supported experimental loads align inconsistently with FEA predictions with $22 \mathrm{~N}$ for a 10 -ply laminate up to $118 \mathrm{~N}$ for a 28 -ply laminate yet provided adequate baseline for load magnitudes.

Figures 4.29-4.30 for DA409 and TR50S are similar to the previously mentioned figures except are nondimensionalized to allow snapthrough load predictions for laminates of various $L^{*}$ ratios. Nondimnensionalized forces were found through Equation 4.5, with $E_{1}$ in Gigapascals. For example, when the snapthrough actuation load is required for a TR50S laminate that has $0.314 \mathrm{~m}$ square sides, 8-ply, fixed to a wall as a panel, and $L^{*}=196$ the corresponding $F^{*}=0.65$. This $F^{*}$ value corresponds to an actuation load of $F=9$ N.

$$
F^{*}=F /\left(E_{1} L^{2}\right)
$$




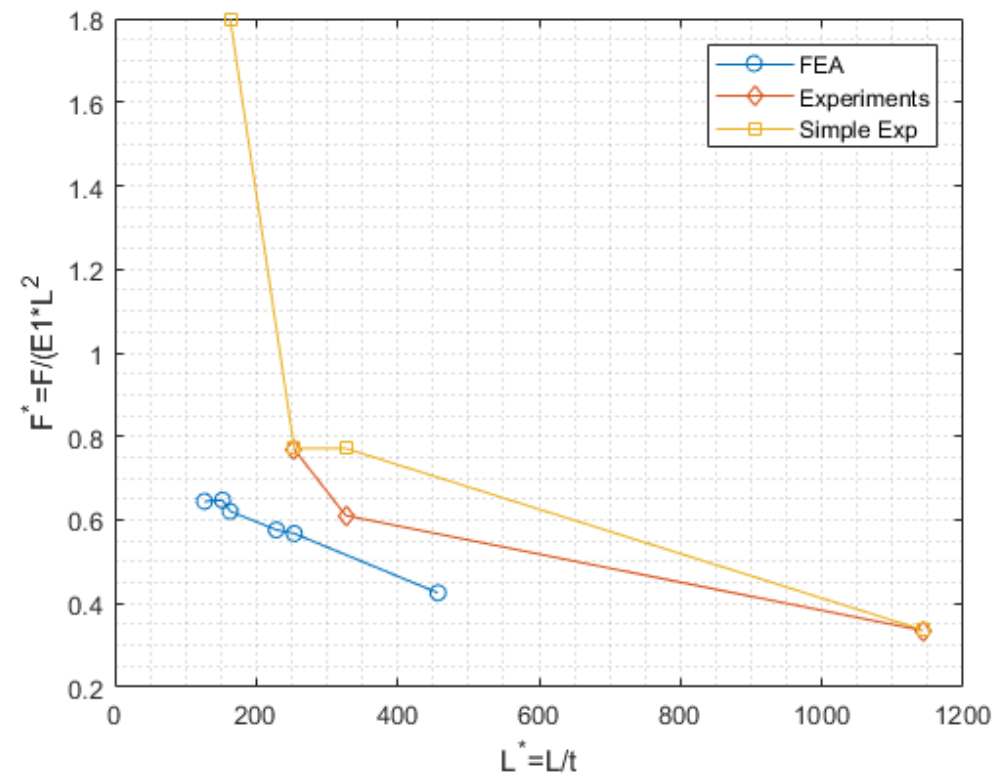

Figure 4.29: Nondimensional Snapthrough Actuation Load Curve for DA409 as a Function of Number of Lamina (n).

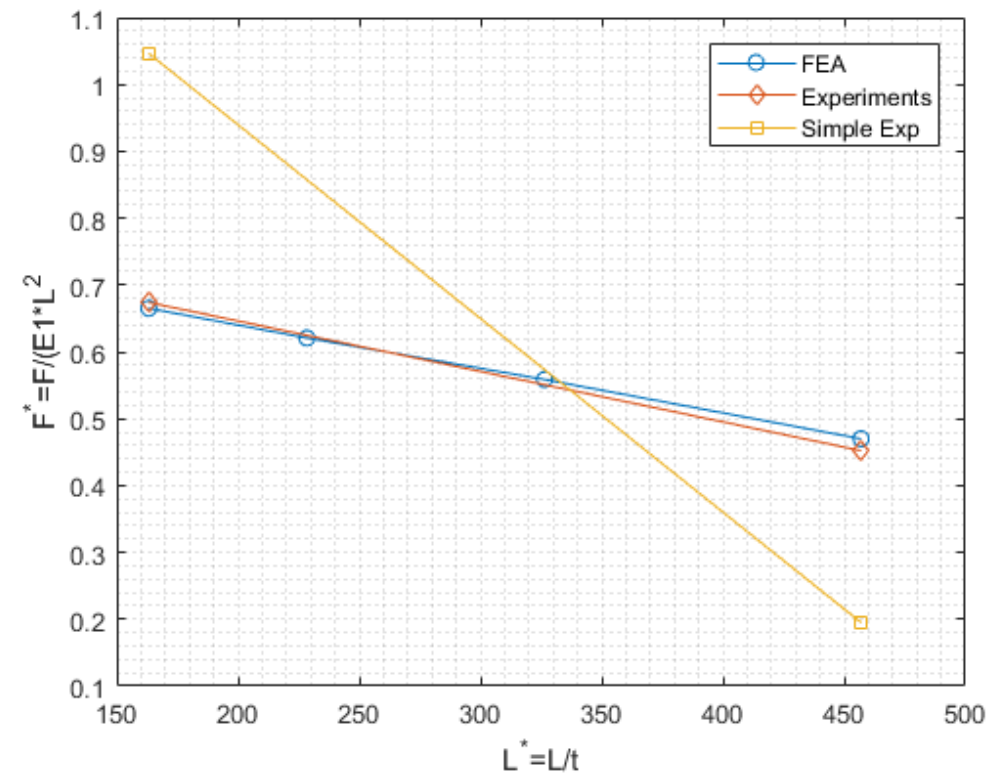

Figure 4.30: Nondimensional Snapthrough Actuation Load Curve for TR50S as a Function of Number of Lamina (n). 


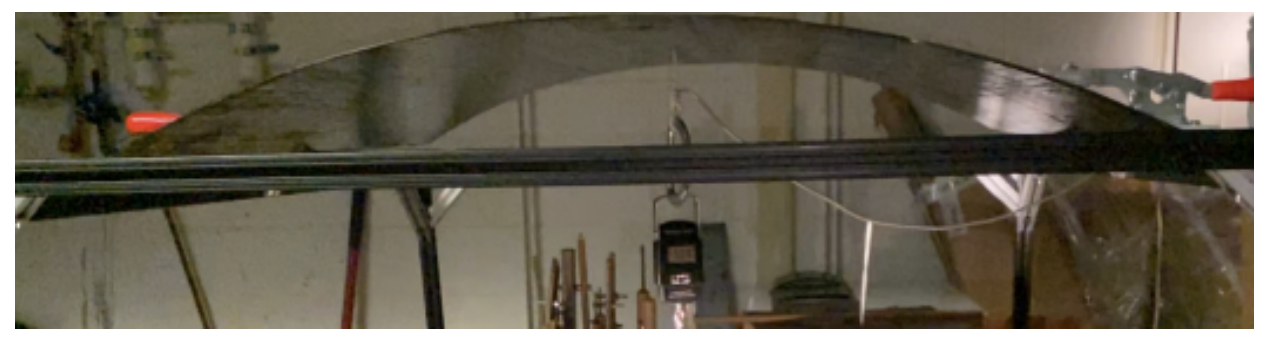

Figure 4.31: TR50S 10 Ply Experimental Cure Shape in Clamped Test Stand with Loading Apparatus to Centroid.

\subsubsection{Observations}

An observation was that drilling $4.0 \mathrm{~mm}$ diameter holes in laminates reduced the shape of samples. In some experiments, where holes larger than $5.0 \mathrm{~mm}$ were made, bistability was lost altogether. This is because the laminates bistability is based on the development of residual stresses and creating a hole in the middle of laminates releases some of the residual stresses, and likely reducing the actuation loads. Additionally, it was determined that the region directly affected by the holes drilled was equivalent to areas ranging from $30 \mathrm{~mm}$ by $30 \mathrm{~mm}$ up to $40 \mathrm{~mm}$ by $40 \mathrm{~mm}$, thus providing the area of effect in FEA.

Another observation during snapthrough was the presence of intermediate modal deformation shapes during snapthrough, similarly as found by Cantera et al. When observed using slowmotion video capture and after the laminate snapthrough had been actuated, multiple intermediate modes could be seen, as depicted in Figures 4.31-4.34. Figure 4.31 is the undeformed, unloaded equilibrium cure shape of a TR50S 10-ply laminate placed in the test-stand. Figure 4.32 is the same laminate after $67 \mathrm{~N}$ have been used to actuate snapthrough and deformation begins. Figures 4.33-4.34 are continuation of the laminate exhibiting two additional intermediate modal shapes. These modes could be described as having some amount of twist (Figure 4.34) or possessing the quality of being a scaled version of the saddle shape solution (Figures 4.32-4.33). It is possible that the additional solutions generated by the Rayleigh-Ritz analytical approach, where twist $\left(w_{11}\right)$ did not equal to zero, are descriptors of these modal shapes. Moreover, these modal shapes could be generated in FEA using Linear Perturbations (Figure 4.35) or Static:riks and eigenvalue solutions to provide comparison. On the other hand, the modal shapes that contain twist could be attributed to the clamps being positioned slightly off center. Modal solutions from Linear Perturbations are shown in Figures 4.35 for comparison. 


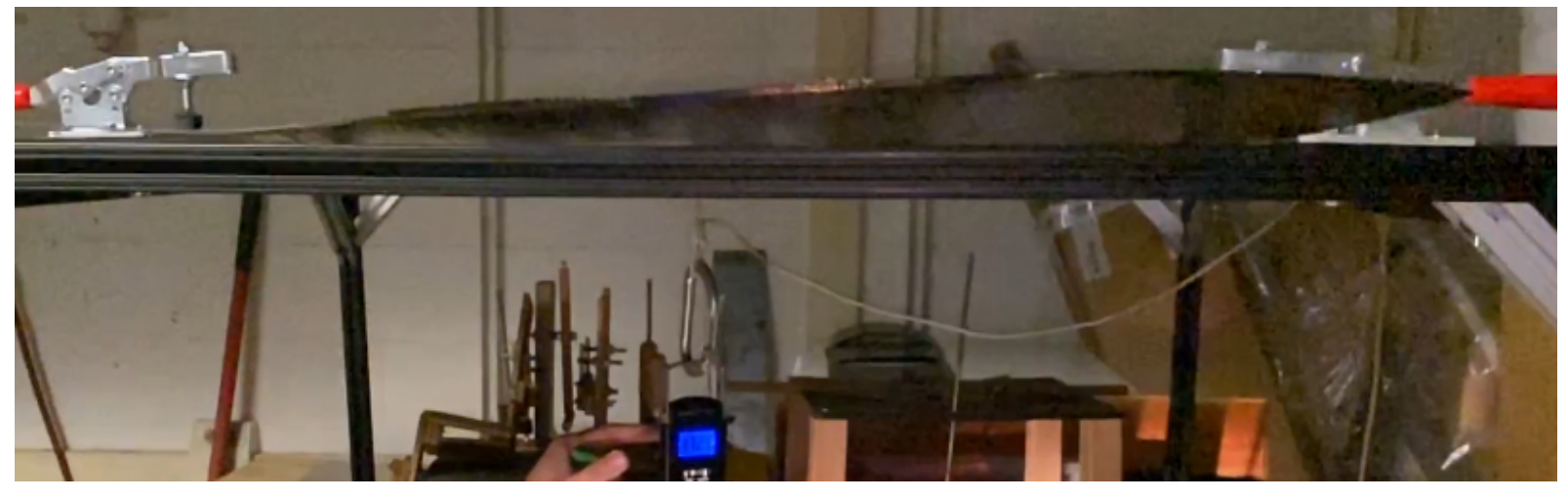

Figure 4.32: TR50S 10-Ply Experimental Intermediate Modal Shape A.

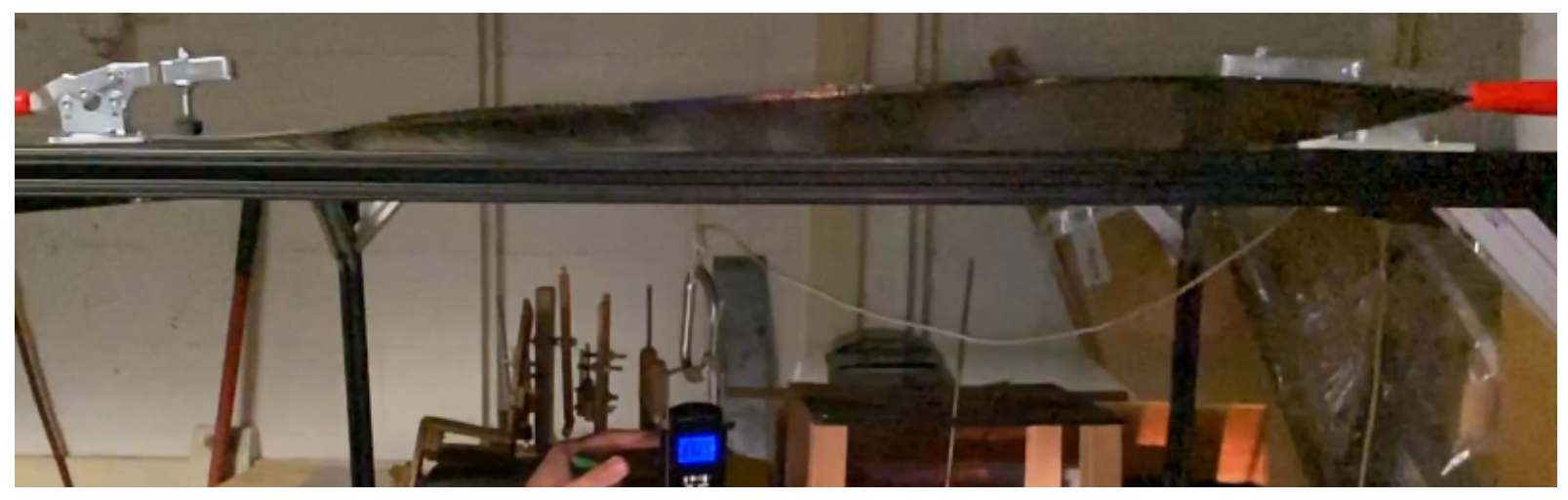

Figure 4.33: TR50S 10-Ply Experimental Intermediate Modal Shape B.

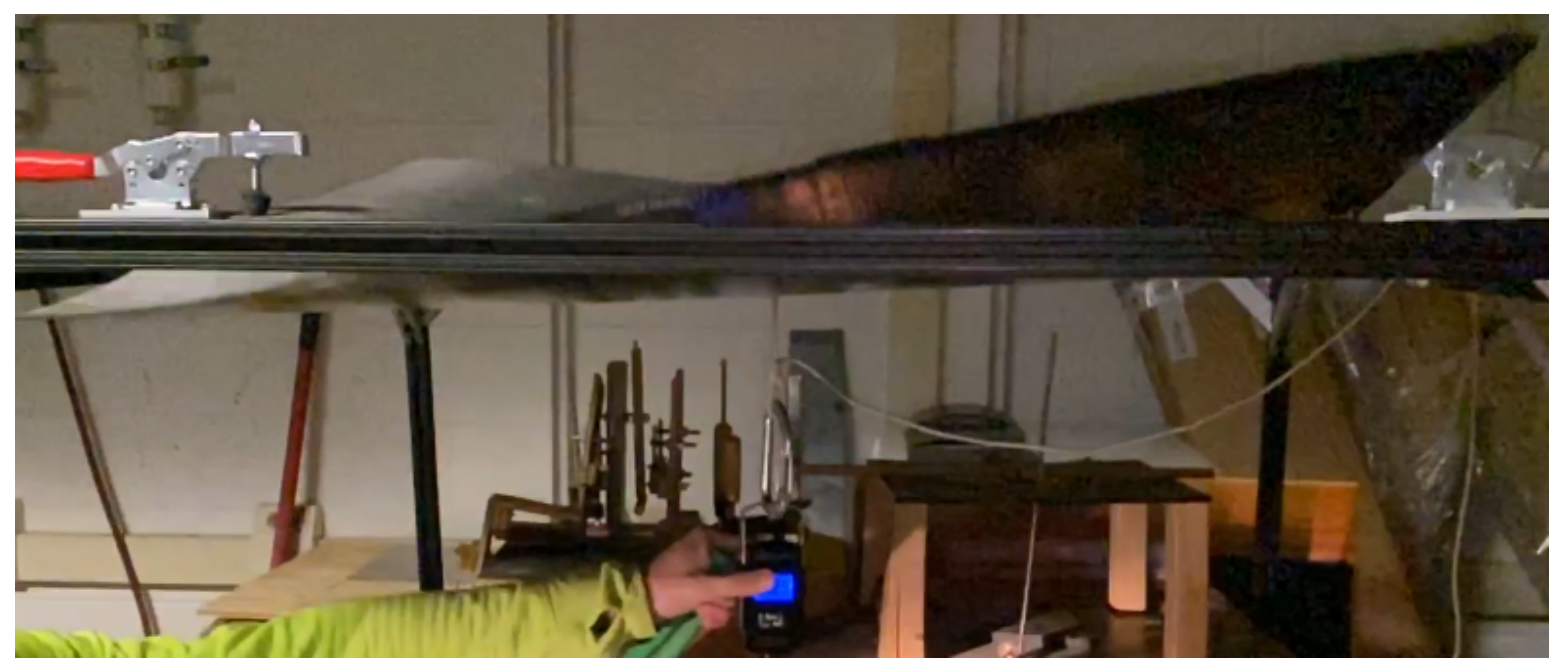

Figure 4.34: TR50S 10-Ply Experimental Intermediate Modal Shape C. 


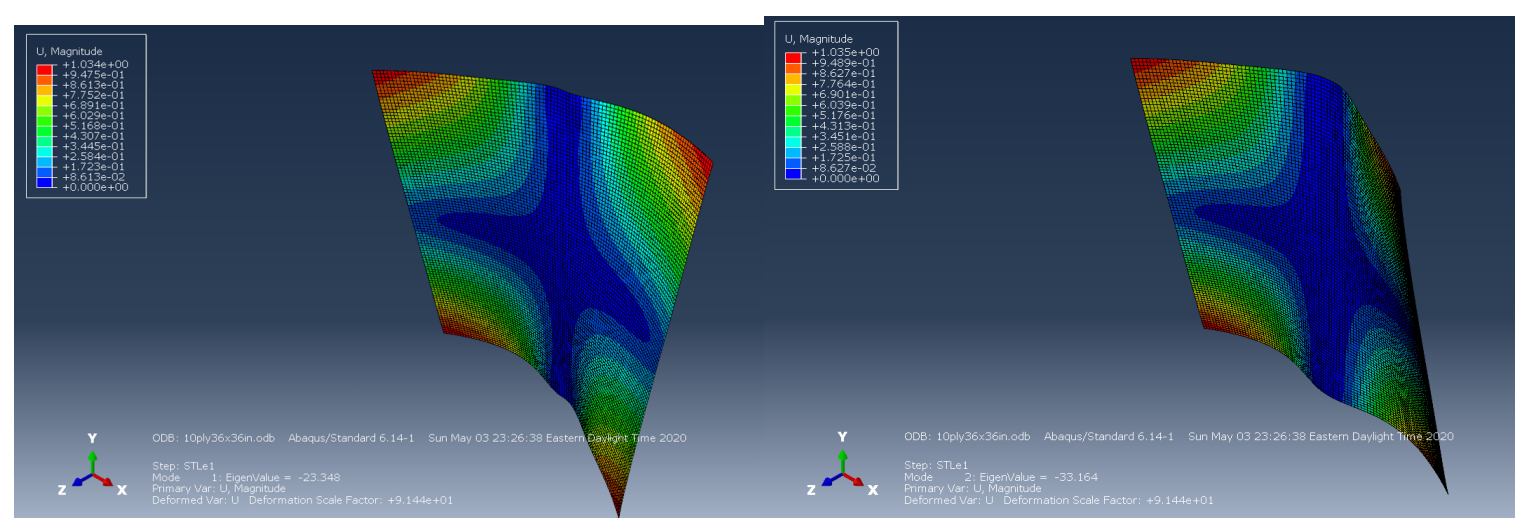

(a) Modal Solution 1 Obtained under 23N Applied Load. (b) Modal Solution 2 Obtained under 33N Applied Load

Figure 4.35: FEA Generated TR50 Linear Perturbation Modal Solutions for a 10-ply Laminate.

Another observation concerning the integration of a metal stiffener to increase the snapthrough force for the laminate. This observation came more form an accident than was performed on purpose but in an instance a laminate remained stuck to the $25 \mathrm{~cm}$ mold plate with the plate taking a curve shape of the laminate (Figure 4.36). Deflection and curvature measurements were not taken but rough force estimates were done to determine how much force was needed to actuate snapthrough. The laminate in question was already thick (30 plies) but upon applying $660 \mathrm{~N}$ to the laminate, it did snapthrough but upon release of the weight, the laminate returned to its original shape. This suggested that the laminate was unstable; however, it served as anecdotal evidence that stiffening agents could be added on the outside of a thick laminate to increase snapthrough loads.

\subsection{Sources of Error}

As has been stated by Dano, Hyer, and other researchers have found before, environment and material have effects on the critical point. It was found that different batches of prepreg unitape yielded mixed results for experimental critical points and thus provided complications for making consistent descriptions. Brampton et. al stated that CFRP material properties can vary significantly due to uncertainties their determination affecting the Young's moduli, and thermal expansion coefficient [17]. Furthermore, these uncertainties continue into the ply thickness, variation in density of unitape fibers, presence of voids in the laminate, and temperature differential in the oven. Hyer stated that even a $1 \%$ variance in thickness could have influential effects on the critical 


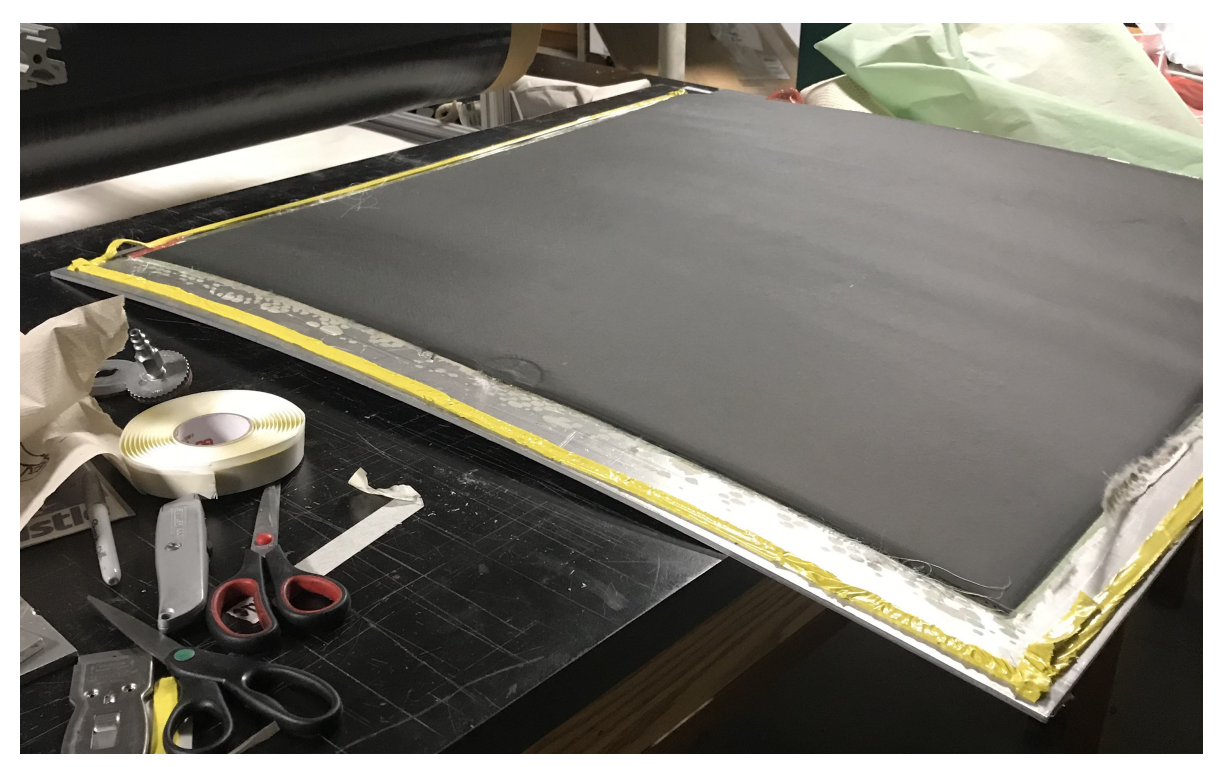

Figure 4.36: 28-Ply TR50S Sample Accidentally Bonded with Mold Plate.

point and that bistable laminates are very susceptible to moisture ingress [24]. The effects of moisture ingress were observed as samples developed loss of bistability 1-3 days after fabrication. To minimize this, attempts were made to gather all data from samples within one day and DA409 samples were reheated to evaporate moisture. However, the same preventative measure could not be done for TR50S samples since they would actually become unstable. The final source of error was due to the nature of using hand layup procedures which allowed for variation in dimension, surface defects (Figure 4.35), and lamina misalignment relative to principle material axes.

\subsubsection{Lamina Thickness Study}

An addition study was done out of curiosity to determine the role of thickness in the determination of bifurcation critical points. The study looked at lamina thicknesses of $0.12 \mathrm{~mm}$ and $0.20 \mathrm{~mm}$ where $0.12 \mathrm{~mm}$ was the manufacturer listing for the prepreg material and $0.20 \mathrm{~mm}$ was the average thickness as measured in cure samples. All other DA409 material properties and constants were kept the same for FEA analysis. Results are shown in Figure 4.38 for the nondimensionalized curvature with the subfigure (a) using $0.12 \mathrm{~mm}$ and subfigure (b) using $0.20 \mathrm{~mm}$. For a $50 \%$ increase in lamina thickness, the critical point moved from $L_{0.12}^{*}=70$ to $L_{0.2}^{*}=73$, corresponding to a 62-ply laminate using $0.2 \mathrm{~mm}$ lamina. This implies that lamina thickness does not have as signifi- 


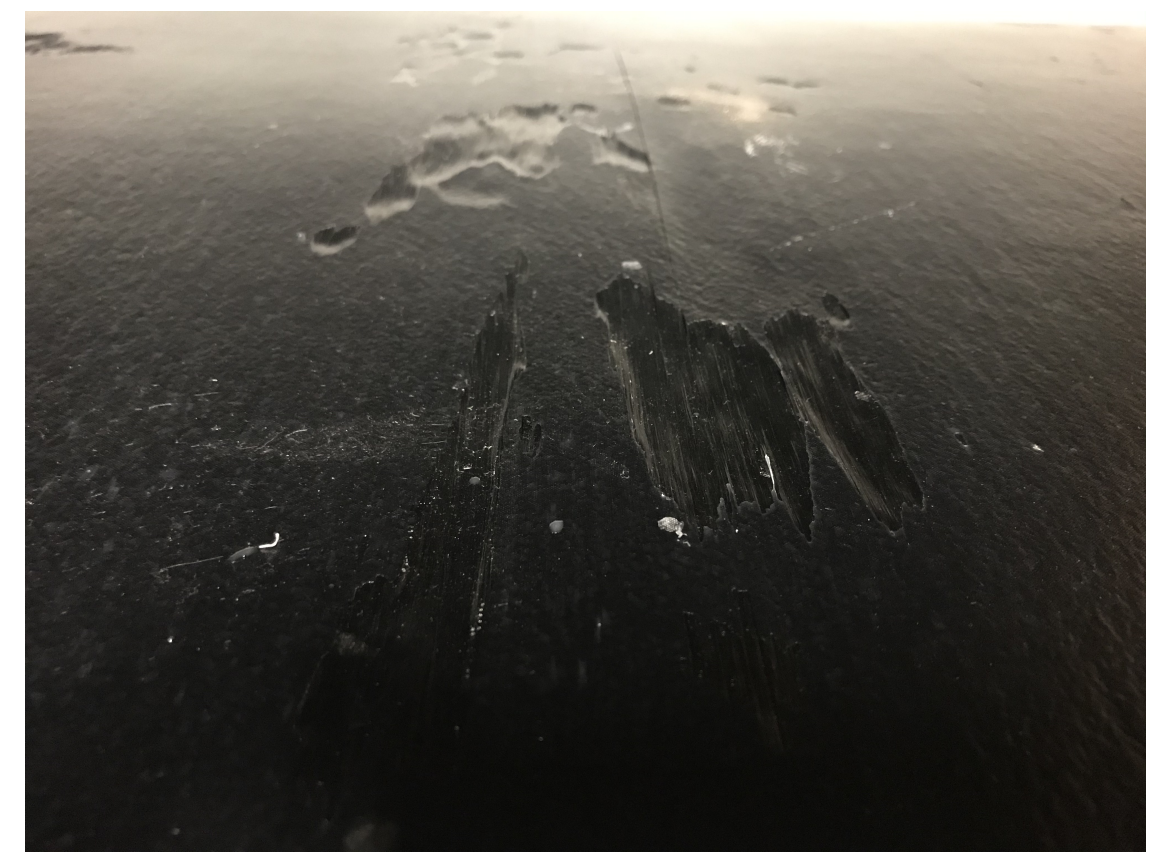

Figure 4.37: Sample Surface Defects for a DA409 Laminate.

cant a role as expected in determining the location of the critical point and supports the facet that FEA predictions for shape require inclusion of polymer relaxation mechanics, and material imperfections and/or bifurcation is not an appropriate method. However, from Figure 4.23, it is known that FEA has been predicted high deflection solutions, implying that FEA may yield better results from an IMPERFECTION command in Abaqus. The IMPERFECTION command scales the nodal displacement geometry of the cure shape, as was used by Tawfik [21].

\subsection{Conclusion of Results}

Determining the loss of bistability with bifurcation plots generated from FEA was inconclusive as FEA suggested bistabilty would occur until greater than 60 plies for both DA409 and TR50S. When this data is compared to experimental observations, it can be seen that this is simply not true. Additionally, when comparing this data against what is found in literature, FEA bifurcation plots appeared unreliable. On the other hand, the energy landscape appears to provide better analysis for the expected prediction of bistability loss and FEA provides good predictions for snapthrough loads. Rayleigh-Ritz gives better results for prediction of bistability but remains as a generalized 


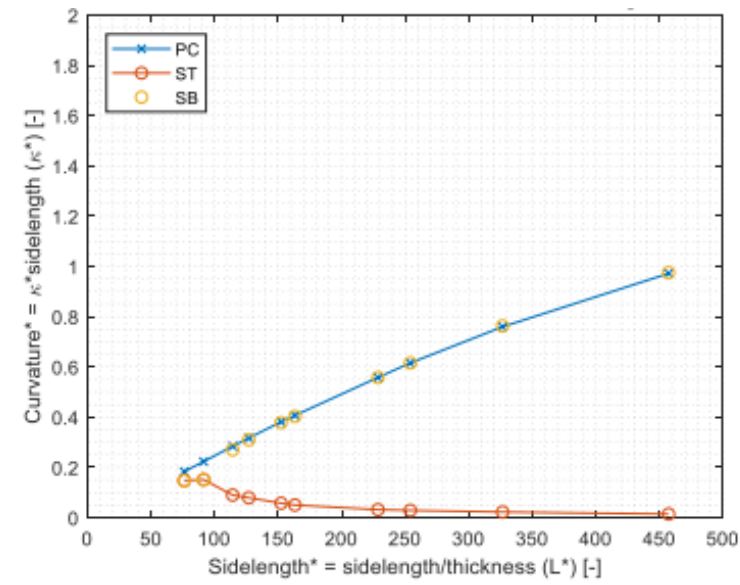

(a) $0.12 \mathrm{~mm}$ Lamina, $L_{\text {critical }}^{*}=70$.

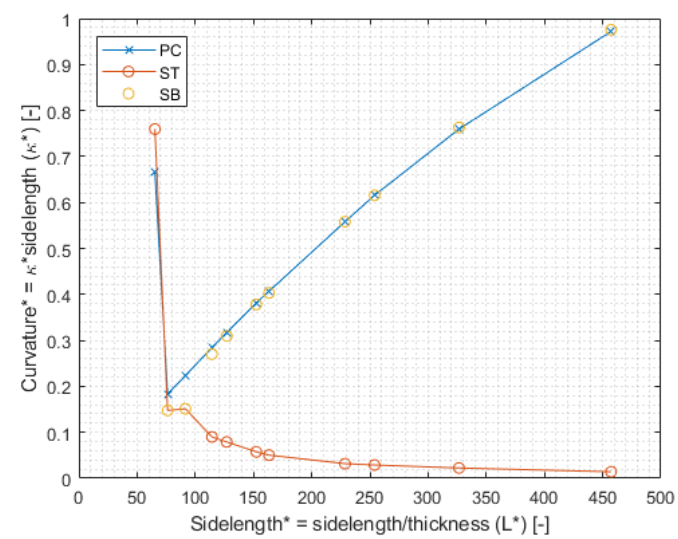

(b) $0.20 \mathrm{~mm}$ Lamina, $L_{\text {critical }}^{*}=73$.

Figure 4.38: FEA Generated DA409 Bifurcation Plots Examining the Effects of Lamina Thickness on Results.

prediction solution. Bifurcation results, shape, and snapthrough appear significantly dependent on lamina thickness. Yet, the lamina thickness study indicated for FEA that thickness did not influence the critical point as much as previously thought. Furthermore bifurcation plots allow the prediction of general shape for a variety sidelength and thickness combinations of laminates specific to each material. Snapthrough load plots provide prediction for specific materials but again can be used for a variety of sidelength and thickness combinations after the load-lamina plot is nondimensionalized. 


\section{Chapter 5}

\section{Conclusions and Further}

\section{Discussion}

Thick bistable laminates were of interest due to the theoretical increased stiffnesses and snapthrough loads, as well as the versatility in creating larger plates. A literature review was done to determine the extent of research done on thick multistable and bistable laminates. Several analytical and finite element analysis approaches have been used to describe bistable laminates. Analytical models rely heavily on a Rayleigh-Ritz analysis of the first variation of the total potential energy of the laminate. However, the majority of shapes studied using this technique were rectangular. FEA was stated to have better shape prediction results for a wider variety of shapes and to be more reliable in boundary prediction and snapthrough load prediction. Thus, FEA has been used to study the behavior of non-traditional shapes. Finally, it was found in literature that a key length-to-thickness aspect ratio of 152 defined the edge of bistability.

From these findings, this thesis looked at using a more computationally simplistic RayleighRitz approach to determine the general cure shapes of thick bistable laminates. The method was first developed by Hyer and is based on strain assumptions. Further, finite element methods were also used to model the cure shape, predict the bifurcation critical point and determine the snapthrough loads in order to provide additional points of reference. Bistability, deflection, curvature, energy landscapes, and abnormal phenomena were examined. 


\subsection{Answering the Research Question}

The goal of this research was to provide more information on the design space for implementation of bistable laminated composites. As stated numerous times, several modeling methods were used to create predictions on bistable behavior by providing descriptions for deflection shape and curvature at equilibrium configurations in addition to load studies for two different boundary condition scenarios. Descriptions for deflection were desired in order to grasp, in a quantifiable manner, the amount of physical space that a bistable laminate would occupy. Curvature was also important because it allows the determination of the concavity of laminates, and the amount of deviation from the mold surface. Snapthrough behavior studies, both observations on how a laminate is moving between equilibrium configurations, and the loads required to cause the laminate to shape-morph are equally as important.

The models allowed reasonable descriptions for the shape parameters to be identified and a graphical relation was provided for determining the deflection and curvature for bistable laminates of varying sizes. The reliability of these prediction plots was compared against experiments and it was evident that, roughly speaking, both models were effective. However, modeling predictions sometimes provided significant percent differences when compared to samples. Each model excelled at different physical predictions. Rayleigh-Ritz was more effective in determining the bifurcation point predicting a loss of bistability at 36 plies, or a sidelength-to-thickness ratio of $L_{\text {critical }}^{*}=127$. Although, it should be noted that Rayleigh-Ritz only used a generalized paraboloid to model the thermal deformation. As such, the paraboloid is limiting because it cannot capture the exact cure shape over the entire domain. Even though the results here show that Rayleigh-Ritz predicted parabolic edges, it is probably more of a coincidence. Despite this, Rayleigh-Ritz yields reliable general shape predictions assuming an average percent error of 19.5\%. Increasing the power of the polynomial would decrease the percent error but would also increase computation solve times for marginal benefit. On the other hand, finite element analysis was better at determining cure shapes than Rayleigh-Ritz due to the fact Abaqus was able to capture edge conditions and variability between equilibrium shapes. Experimental validation agreed by averaging $18 \%$ difference for deflection predictions with a dissimilar measurements for curvature prediction. Additionally, FEA was able exhibit $11 \%$ average difference between equilibrium shapes, in terms of peak deflections and was verified experimentally. However, curvatures on average were predicted to be much lower than 
found from experimental samples. This is believed to be a result of the sudden increase in measured nodal deflection for an experimental saddle shaped laminate, which was included in the averaging scheme or the result of material defects during fabrication. Even though curvature predictions may not have been as close in magnitude as Rayleigh-Ritz, FEA did provide deflection and curvature measurements anywhere in the laminate domain. Predictions for deflection and curvature can be done by multiplying the two laminate principle axis deflection equations. However, the product of the average of two principle axis curvatures (akin to Gaussian curvature) is only an approximation and better FEA shape curvature results would result form taking into account all nodes modeled. Prediction of bistability loss made by FEA was ambiguous in nature. Bifurcation plots suggested laminates containing upwards of 70 plies were needed to be sufficiently thick to eliminate bistable behavior. From experiments, this is known to not be true. Examination of the energy landscapes seemed to provide more insight on bistable behavior. Determining the point at which the laminate does not snapthrough on the energy landscape is easy, and it can be determining at which thicknesses bistability no longer occurs. Using the energy landscape gave results that agreed with the $L_{\text {critical }}^{*}=152$. Furthermore, investigating the lamina thickness also indicated changes in the energy landscape giving the postulation of lamina thickness sensitivity. Thus, it can be said performing a detailed survey of bistable behavior is best done through the use of finite element software, especially if predictions take into account all nodes. The reasoning likely resides in the fact that FEA utilizes a 3D composite shell model whereas Rayleigh-Ritz relies on generalization out-of-plane deformation predictions and extended classical lamination theory assumes plane stress.

A direct comparison between Rayleigh-Ritz and FEA was not completed concerning snap through actuation loads since a method of virtual work was not included for the analytical model as well as claims in literature stating that virtual work was underwhelming. Because of this FEA was the only method used to create descriptions of snapthrough behavior. Snapthrough loads were able to be predicted accurately for TR50S but were not as satisfactory for DA409. This is most likely due to the result that DA409 laminates had an observably higher amount of surface and fabrication defects. Narrow prepreg unitape sheets likely led to the creation of macrovoids when two DA409 sheets were laid next to each other without overlapping. Since, TR50S was provided in continuous and wide prepreg sheets, these macrovoids could be avoided. Moreover, during snapthrough video capture, it was observed that the laminates exhibited intermediate equilibrium shapes. The significance of these intermediate shapes is not known. 
Thus, it can be concluded that the original research intent has been met but with many new questions created. Both of the previously established analyses can provide adequate descriptions but should currently be used with caution or verified with experimental results.

\subsection{Theoretical Implications and Recommendations for Fur- ther Research}

Overall, Rayleigh-Ritz is effective in quick generalized displacement analysis for cure shape and lays out sufficient deflection and curvature predictions. Additionally there may potential for the out-of-plane deflection (Equation 3.2) to adequately capture edge boundary conditions, which was observed to not be possible for thin CFRP bistable laminates. The only downside to utilizing Rayleigh-Ritz is that setting up the analysis and solving for the large nonlinear system of equations can be troublesome. Realistically, there would be a benefit to implementing Mattioni's product of two $P_{4}$ polynomial functions to describe a square laminate since accuracy is increased. Yet, increasing the accuracy of the Rayleigh-Ritz model costs a signficant amount of computation time if the system is even capable of solving 20 or more unknown coefficients, dependent upon assumptions. Additionally, there exists a need to not define the thermal deformation of a bistable laminate by starting with an assumed shape. Perhaps a piecewise Rayleigh-Ritz function over the domain to with separate assumed displacement shapes should be used. Further, in the implementation of virtual work principles to account for boundary conditions is needed to predict snapthrough behavior.

Due to the aforementioned considerations, this is why FEA is likely a better candidate for describing bistable behavior. Additionally, FEA has always been more reliable at edge condition prediction and can be used to predict snapthrough load. Analysis of the $P_{4}$ deflection polynomials gathered from FEA was not done to determine the meaning of the coefficients; however, using a polynomial in place of an averaged single value peak deflection is more practicable to describe the physical space a bistable laminate may take up. The use of the polynomial also allows a parametric definition of curvature to be determined. One benefit is the availability to calculate the Gaussian curvature, which is indicative of concavity.

However, this is probably not the most optimal method to describe the curvature of a laminate. It would be beneficial to the theory paradigm to change the way that curvature is measured for bistable laminates where all nodes within the domain are considered since each node generated 
by FEA would have an effect on the average curvature. Changes in the theory paradigm should also include observations of a "bistable region" until further understanding of bistable mechanics can be determined.

Another method that could be used with possibly better FEA shape prediction results is the STATIC:RIKS procedure along with the IMPERFECTIONS command to determine the modal cure shapes of thick laminates. Thus, cure shapes can be empirically matched with samples and would allow even more accurate snapthrough behavior predictions

Experimentally, more thick bistable laminates samples are needed to provide confident validations. Exploration of the critical point of bistability with minutely varying lamina thicknesses would also be beneficial to determine the sensitivity of the critical point location. Laminates do not necessarily need to be created with large dimensions, but instead with an aspect ratio that allows discretion in minute thickness to be measurable. Another consideration is that bifurcation follows chaos theory and there exists constants signifying predictions for the locations of other bifurcation points. An analysis utilizing chaos theory and the associated Feigenbaum constant may yield useful data for predicting intermediate modal shapes. Furthermore, FEA can be done using nonlinear perturbations analysis which may yield a better understanding of these intermediate equilibra. Another approach to examining bistability is an examination of residual stresses and how they change during snapthrough which may provide the next biggest insight towards understanding bistable laminate behavior. Further, studies on residual stresses may indicate the effects of moisture absorption and allow fatigue studies.

Finally, it is recommended that bistable laminates studies for potential applications be done. Bistable laminates have many potential applications yet there does not exist a plethora of studies showing benefits and/or cons of bistable laminates in such applications. Notably, it is suggested that bistable laminate airfoil aeroelastic studies for wind turbine blades be conducted. 
Appendices 


\section{Appendix A}

\section{Additional Experimental Details}

The purpose of this appendix is to provide additional reference information for the fabrication process used when creating samples using hand-layup and vacuum bagging techniques. As such, please reference the Methodology Chapter for a description of the procedure.

Each of these prepregs came on rolls with disposable backing paper on one side of the sheets to keep the material from buckling during handling. Cutting of prepreg unitape material was done by creating a stand to hold the rolls (Figure A.1). This was done to ensure consistent lengths and cuts of the materials. Scissors were inadequate for cutting the material consistently and all cuts were done using a box razor. Sheets were then placed in a covered area or freezer to reduce exposure to containments and reduce the tackiness of the epoxy until ready for layup.

The mold was cleaned with a scraper and acetone and prepared by the use of FibRelease 1153 mold release (applied via manufacturer instructions) in one to five layers. Consistently and best results were done following the manufacturer's direction by use of a spray bottle. The FibRelease chemical was determined to not be harmful if inhaled in a ventilated room. Following this, a single layer of nylon peel ply was laid on top of the mold to create a desired textured surface finish. Without this layer, laminates would stick or have defects on the mold surface consistent with massive voids and inconsistent spread of resin. Details on this are in the "Sources of Error" in the Results Chapter. Another green peel ply was laid on top of the laminate to create the same textured surface. Red peel ply was then laid on top, followed by breather, and the vacuum bag (Figure A.2-A.3). Figure A.4 shows the $1.016 \mathrm{~m} \times 1.016 \mathrm{~m}$ oven with two shelves and a vacuum line going into the heated cavity. Figure A.5 shows the standard pump provided, which was specifically made for CFRP structures. 


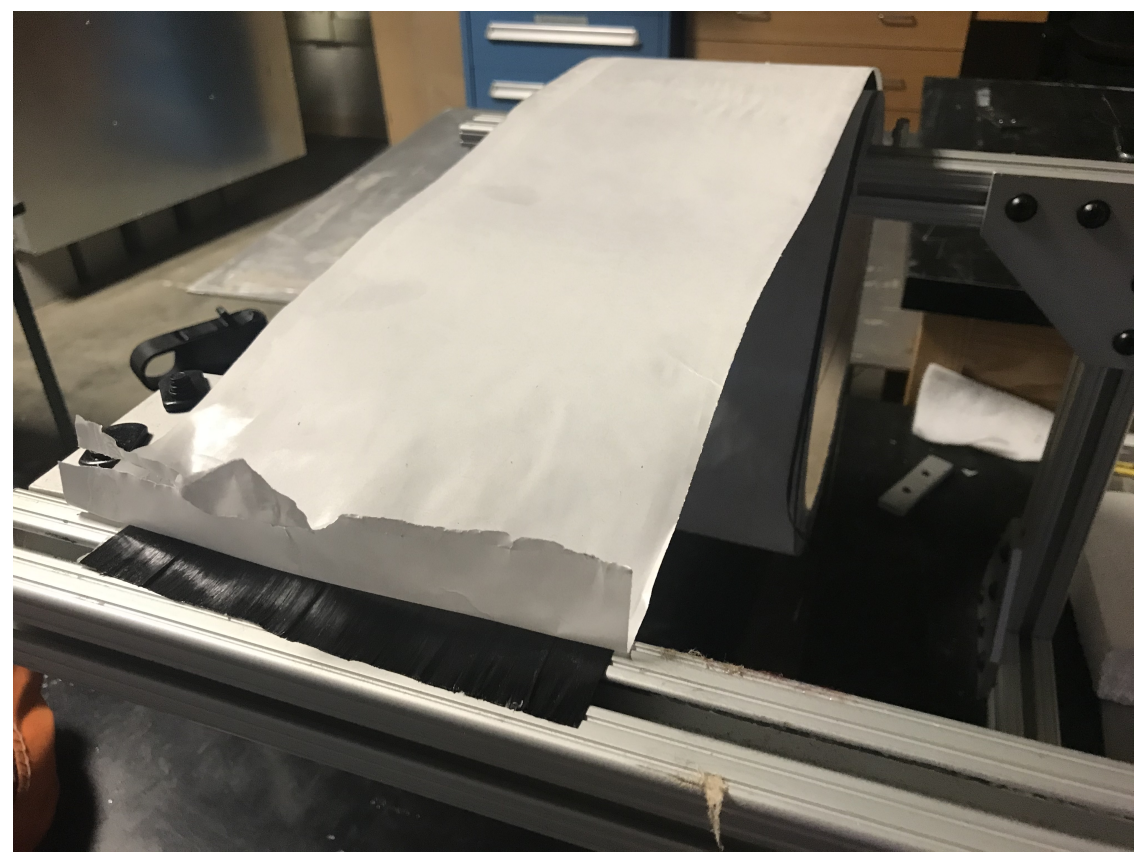

Figure A.1: Stand Used to Increase Consistency of Prepreg Unitape Cutting.

The test stand was created with the use of 1"x1"x1" 80/20 material. The stand situated the test samples approximately half a meter above the floor. Toggle clamps (McMasterCarr 5128A24) with holding capacity of $3 \mathrm{kN}$ under ideal conditions (Figure A.5). Special brackets were fabricated to allow the use of standard 1" $80 / 20$ gussets to mount a plate to hold mount each toggle clamp to the test stand. FEA was done to determined the extend of bending in the clamps and it was determined that the $80 / 20$ material would experience acceptable flexural torsion. 


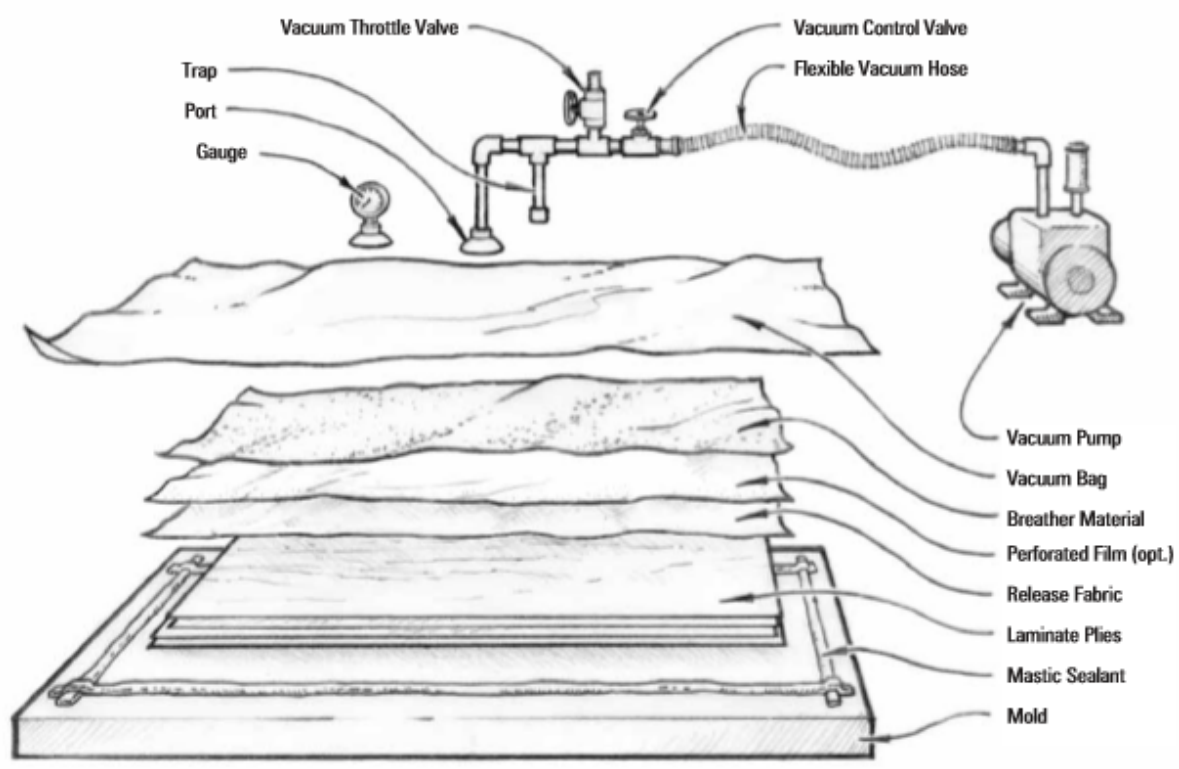

Figure A.2: Typical Vacuum Bag Technique Layup [40]

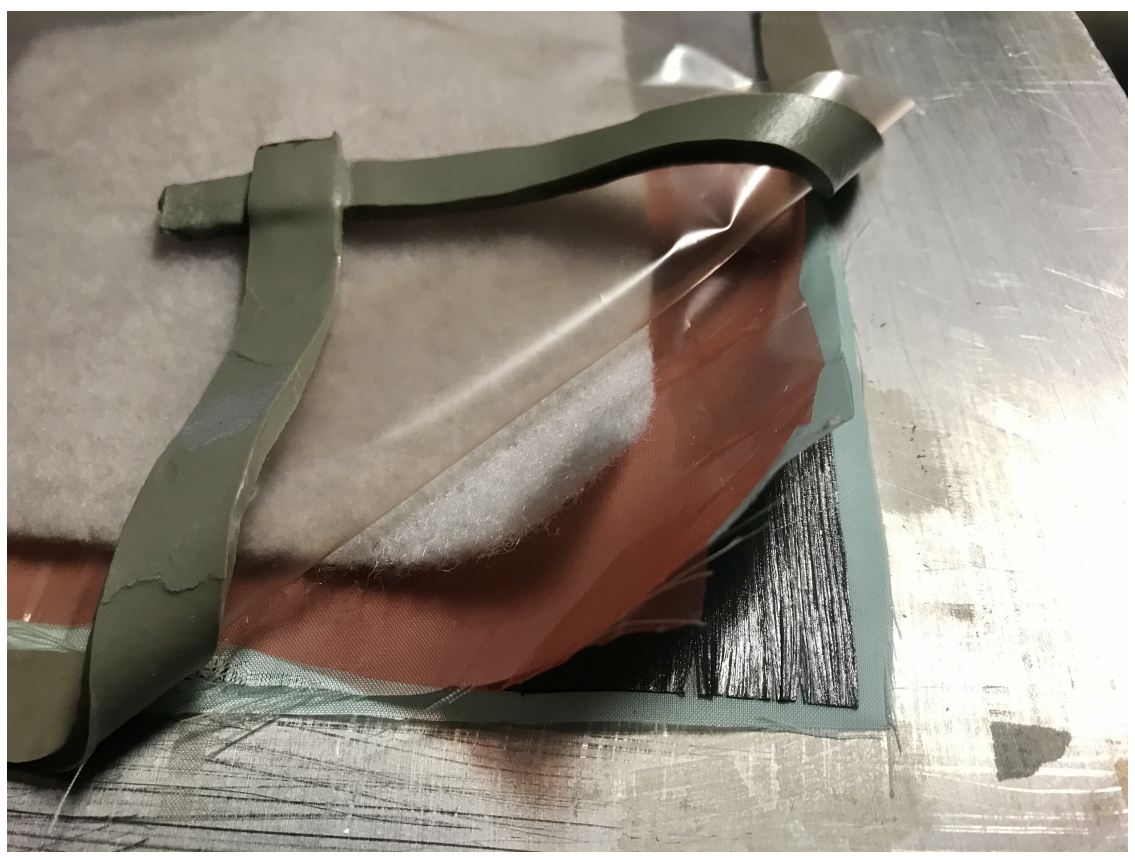

Figure A.3: Vacuum Bag Technique Layup Procedure with Green Peel Ply, Red Peel Ply, Breather, and Transparent Vaccum Bag Sealed with Mastic Tape against the Mold. 


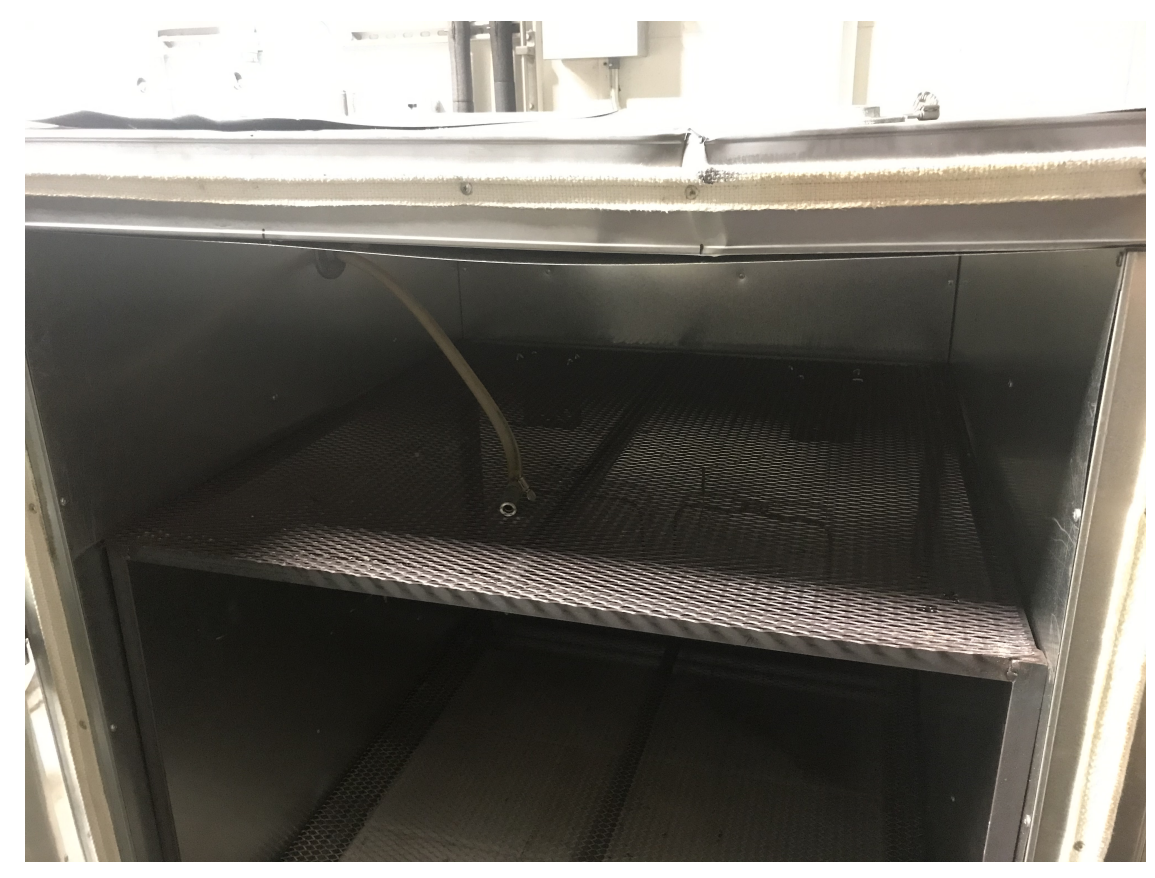

Figure A.4: Inside the Oven. A Hose was Fitted Through the Sidewall to Attach to a Fitting on the Mold Plate.

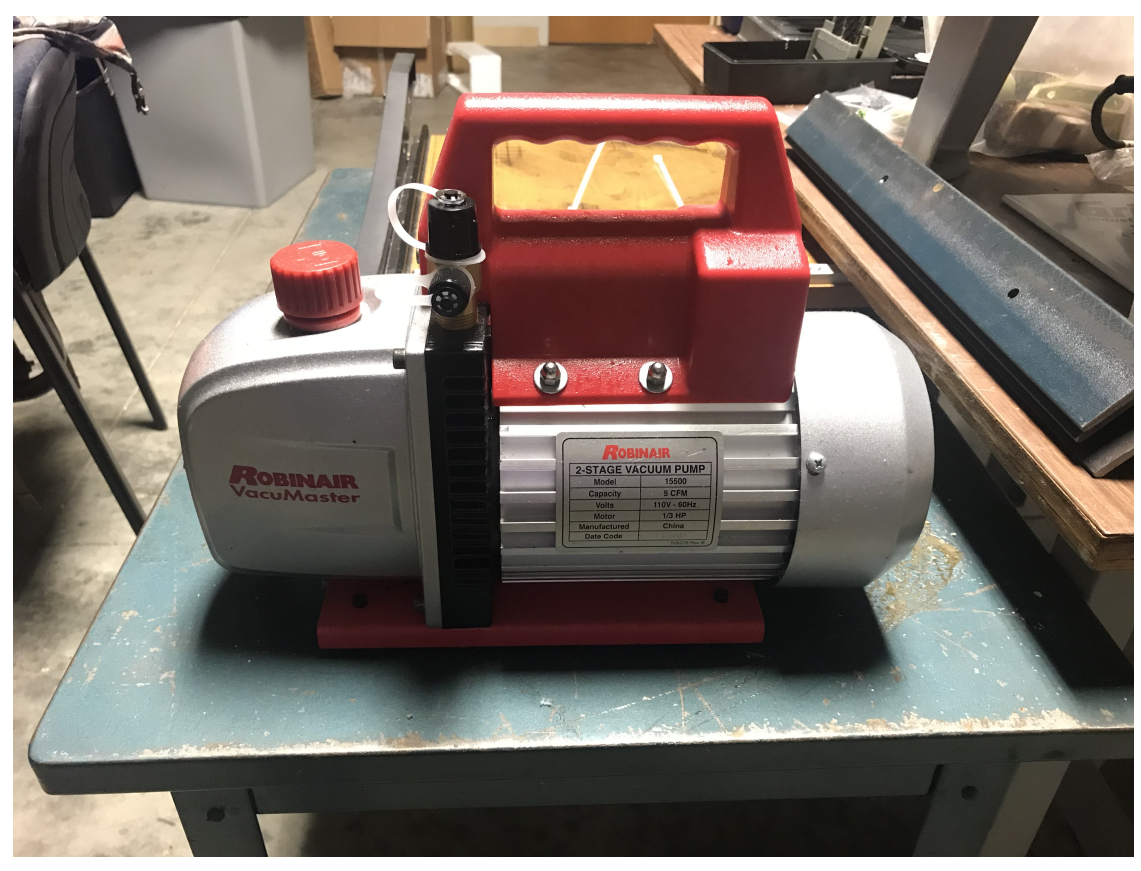

Figure A.5: Vacuum Bag Pump Used. 


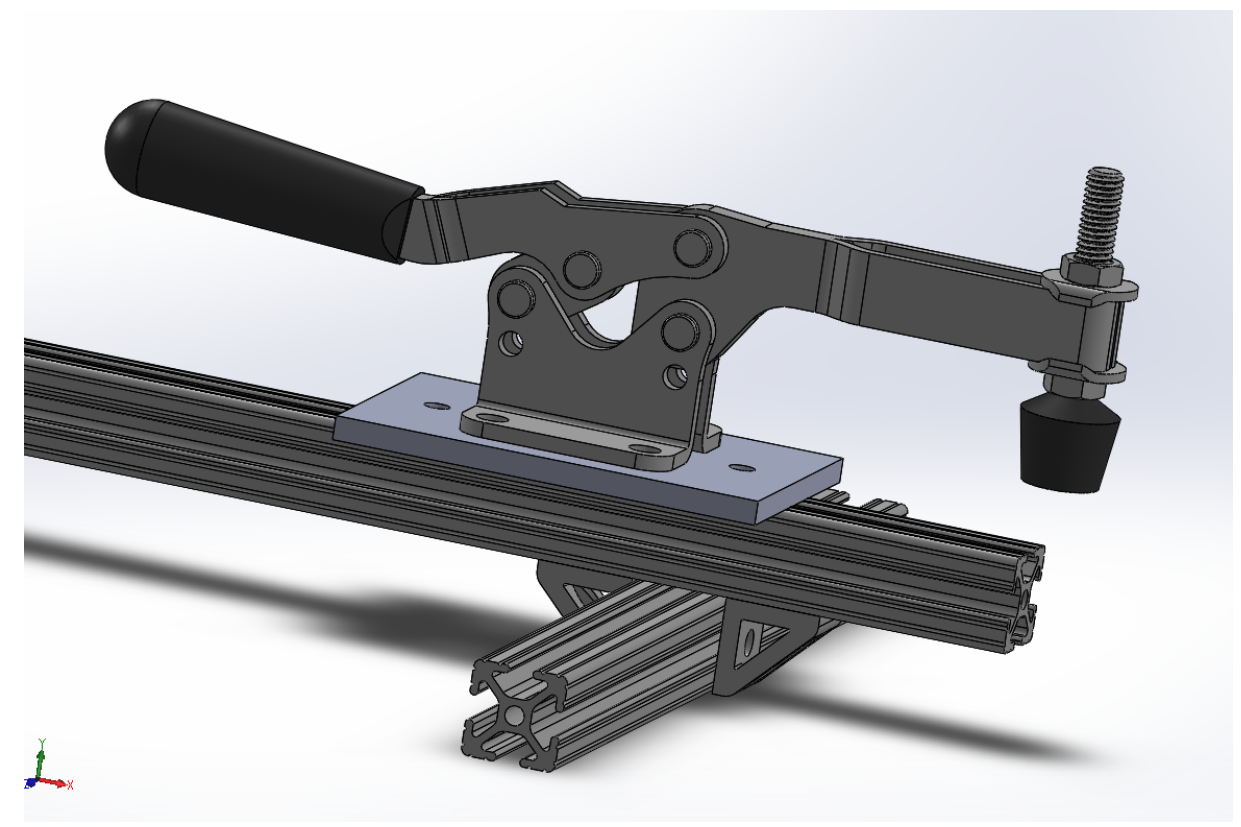

Figure A.6: Toggle Clamp on 80/20 Material Shown Mounted to Bracket.
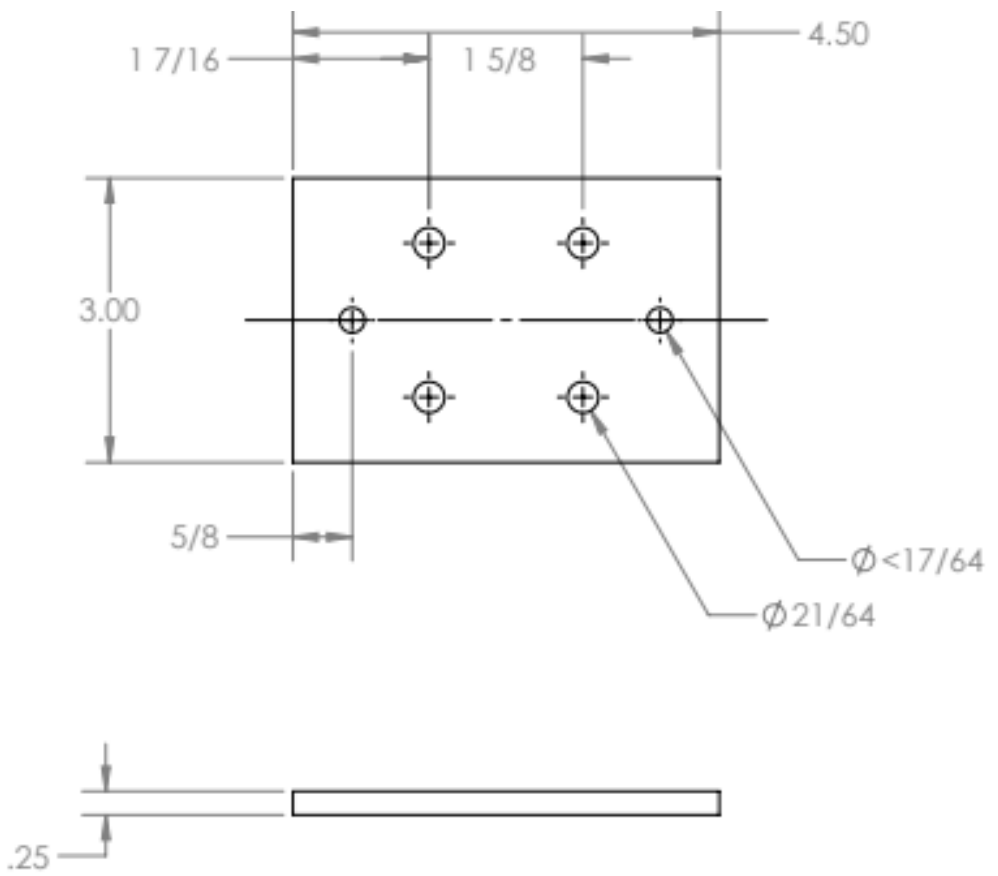

Figure A.7: Toggle Clamp Bracket Designed to Accept 1" Gussets and a McMasterCarr 5128A24 Toggle Clamp. 


\section{Appendix B}

\section{Selected Raw Data and Images}

This appendix is intended to provide a location for the raw numbers used in generating plots as well as images for each laminate modeled.

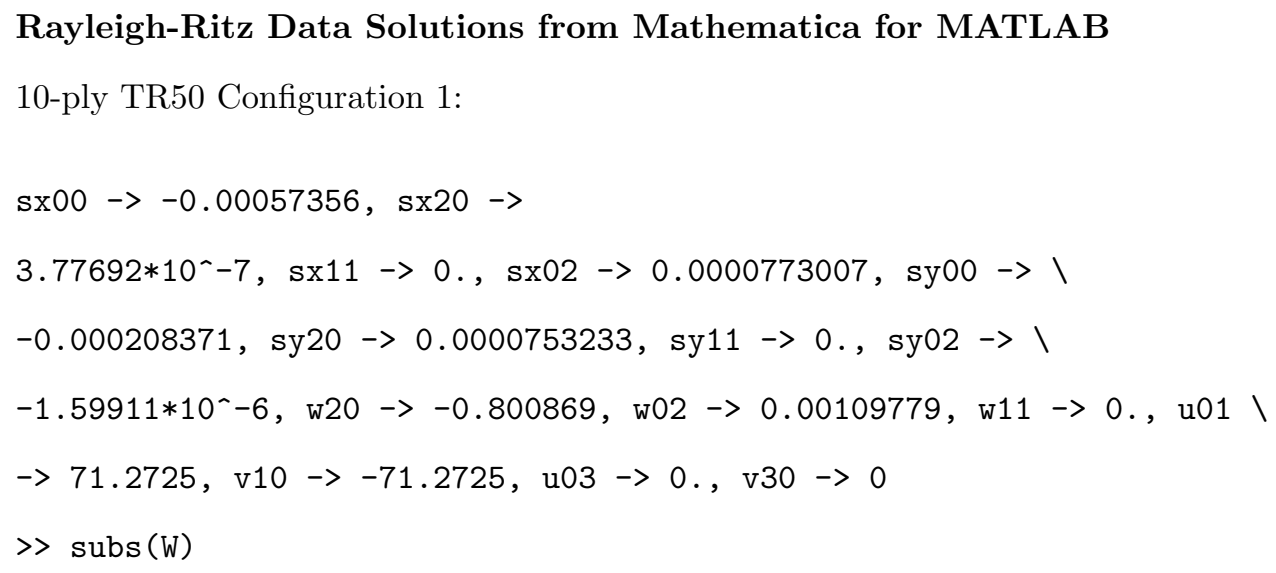

FEA Selected Images 


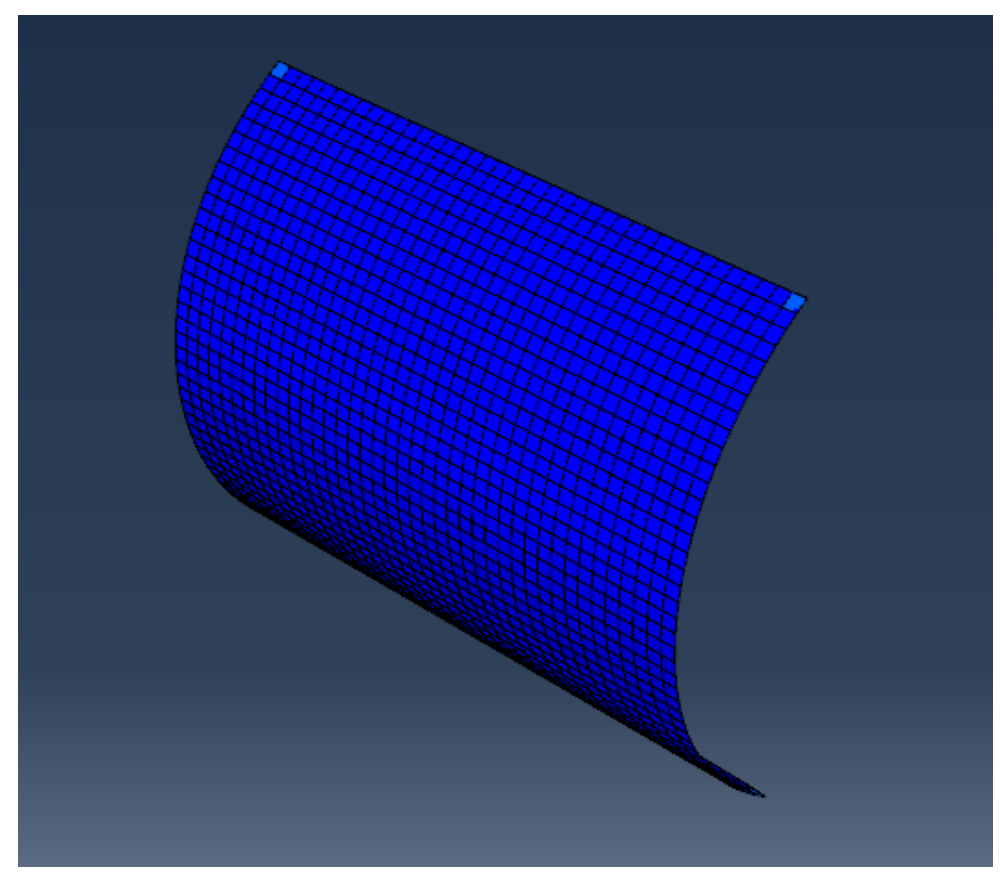

Figure B.1: Cure Shape 10-Ply DA409.

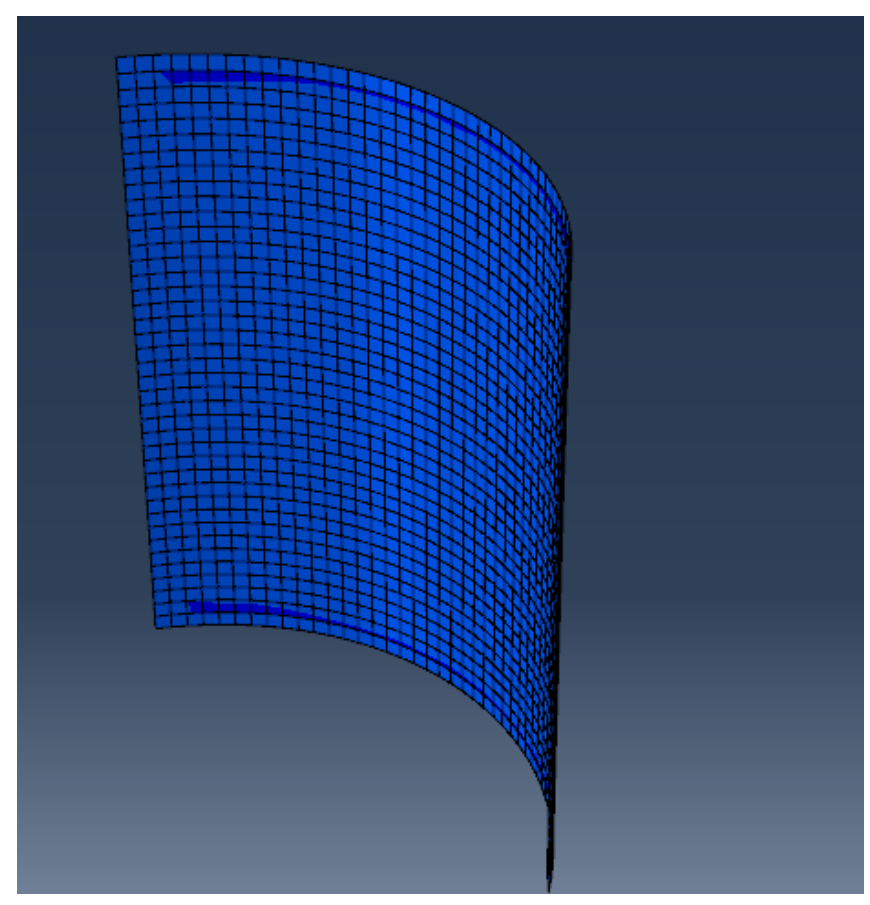

Figure B.2: Cure Shape 10-Ply DA409. 


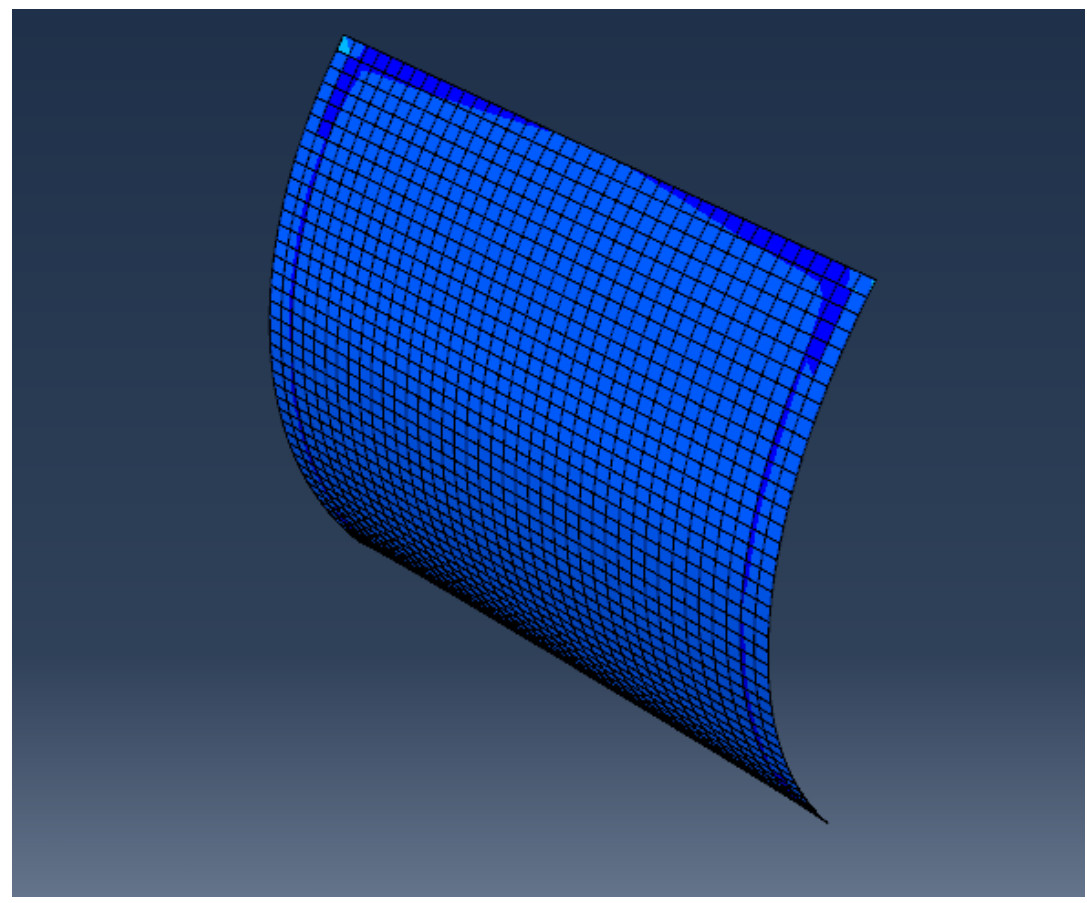

Figure B.3: Cure Shape 14-Ply DA409.

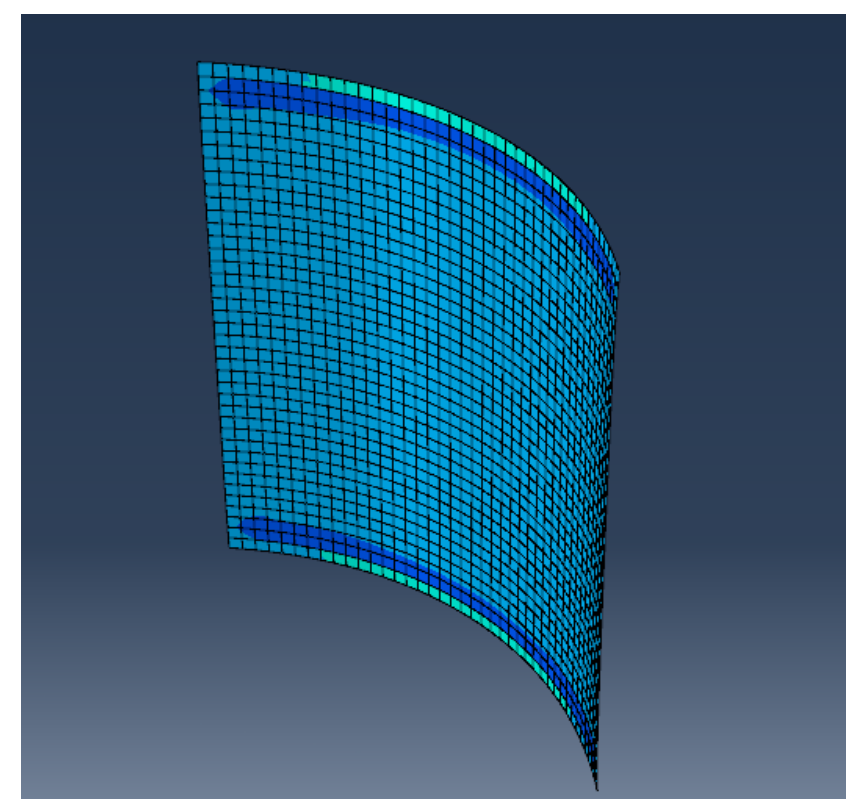

Figure B.4: Cure Shape 14-Ply DA409. 


\section{Appendix $\mathrm{C}$}

\section{Selected Matlab Scripts}

This appendix is intended to provide reference for Matlab scripts created for analysis of data in addition to providing source code for the Rayleigh-Ritz model. The Rayleigh-Ritz model script was made to output the total energy developed, $W$, for the laminate as well as the partial derivatives related to the the definition of the first variation of calculus. For the 15 unknown variables, 15 partial differentiated equations were calculated.

\section{Rayleigh-Ritz Code to Compile System of Equations}

Note: Uses constant curvature calculations.

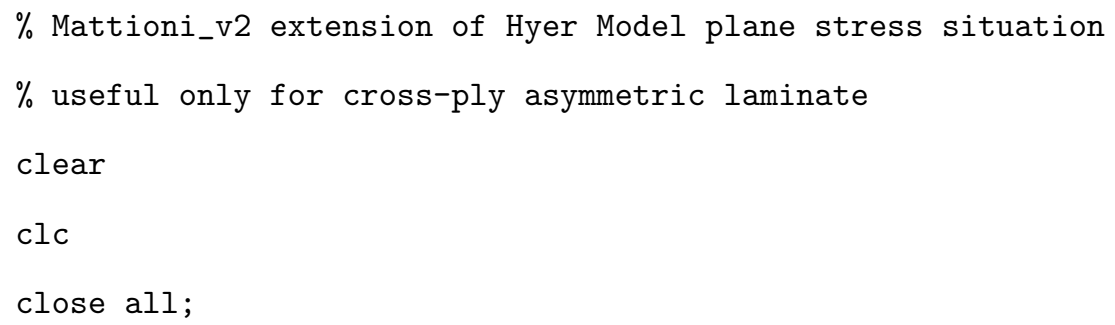




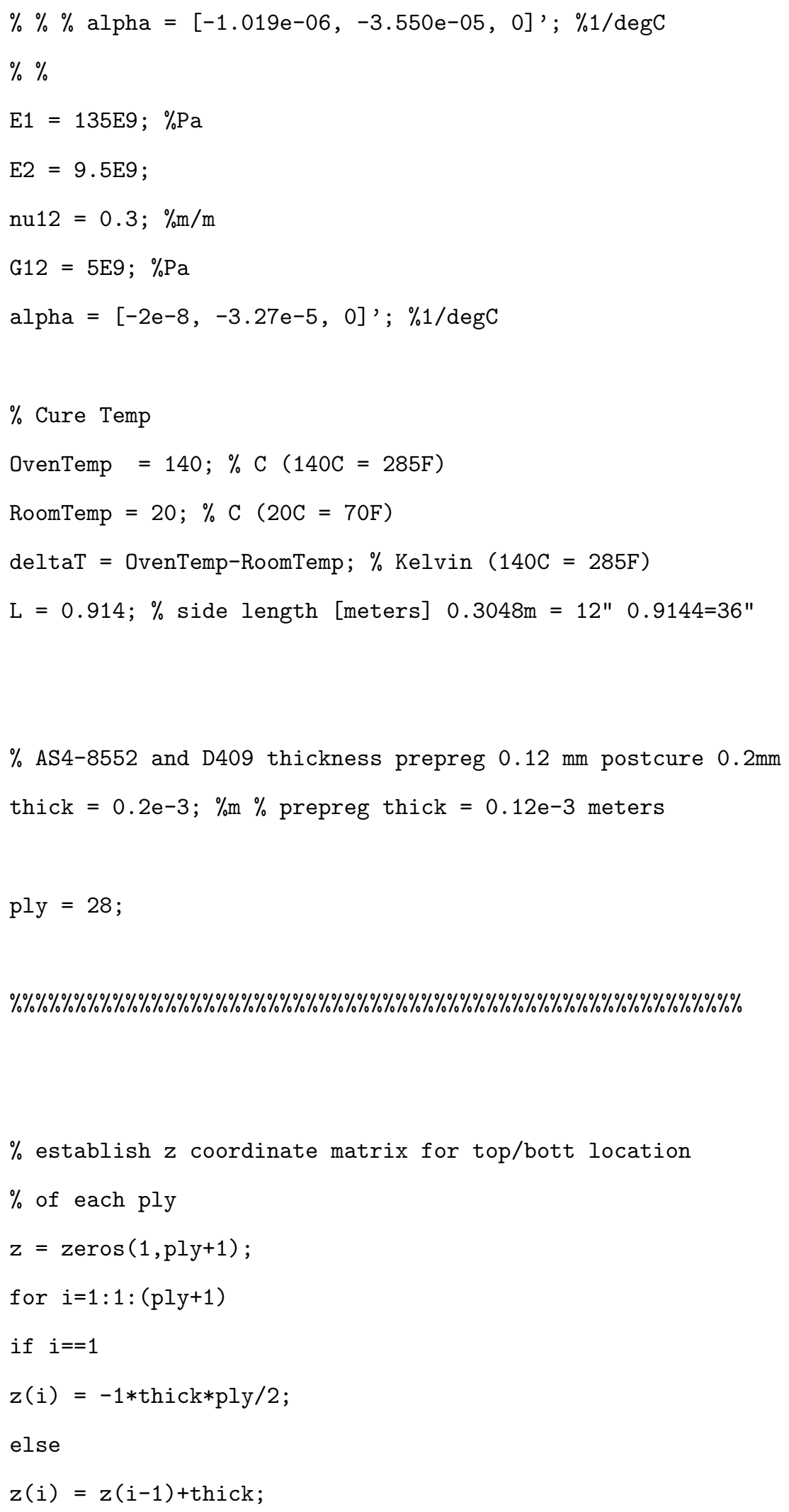


end

end

epsilonZero $=\operatorname{zeros}(3,1)$;

KappaZero $=\operatorname{zeros}(3,1)$;

$\%$ epsilon = epsilonZero+z*KappaZero;

$\%$ matrix

$R=\left[\begin{array}{lllllllll}1 & 0 & 0 ; & 0 & 1 & 0 ; & 0 & 0 & 2\end{array}\right] ;$

$\%$ Reduced Stiffness for a lamina

$Q=$ ReducedStiffness (E1,E2, nu12, G12)

$\%$

Q90 = inv(TransformationMatrix (90)) *Q*R*TransformationMatrix (90)

$\mathrm{Q}=\operatorname{inv}(\operatorname{TransformationMatrix}(0)) * \mathrm{Q} * \mathrm{R} * \operatorname{TransformationMatrix}(0)$

$\%$ Thermal coefficient at 90 degrees

$\%$ alpha90 = R*inv(TransformationMatrix (90)) *inv $(\mathrm{R}) *$ alpha; $\% 1 / \mathrm{C}$

alpha90 = TransformationMatrix (90)*alpha;

alpha = TransformationMatrix $(0) * a l p h a ;$

\% --------- code for Hyer 1981

syms a b c d $x$ y u v w

$\% \mathrm{u} 0=Q(\mathrm{a}, \mathrm{b}, \mathrm{c}, \mathrm{x}, \mathrm{y}) \mathrm{c} * \mathrm{x}-\left(\mathrm{a}^{\wedge} 2 * \mathrm{x}^{\wedge} 3\right) / 6-\left(\mathrm{a} * \mathrm{~b} * \mathrm{x} * \mathrm{y}^{\wedge} 2\right) / 4 ;$

$\%$ v0 $=Q(a, b, d, x, y) d * y-\left(b^{\wedge} 2 * y^{\wedge} 3\right) / 6-\left(a * b * x^{\wedge} 2 * y\right) / 4 ;$

$\%$ w0 $=@(a, b, x, y)(1 / 2) *\left(a * x^{\wedge} 2+b * y^{\wedge} 2\right) ;$

$\%$

$\%$ \%midplane strain

\% fprintf ('---------midplane strains--------- $\backslash n \backslash n$ ')

$\% \operatorname{ex0}=\operatorname{diff}(\mathrm{u} 0, \mathrm{x})+(1 / 2) *(\operatorname{diff}(\mathrm{w} 0, \mathrm{x}))^{\wedge} 2$ 


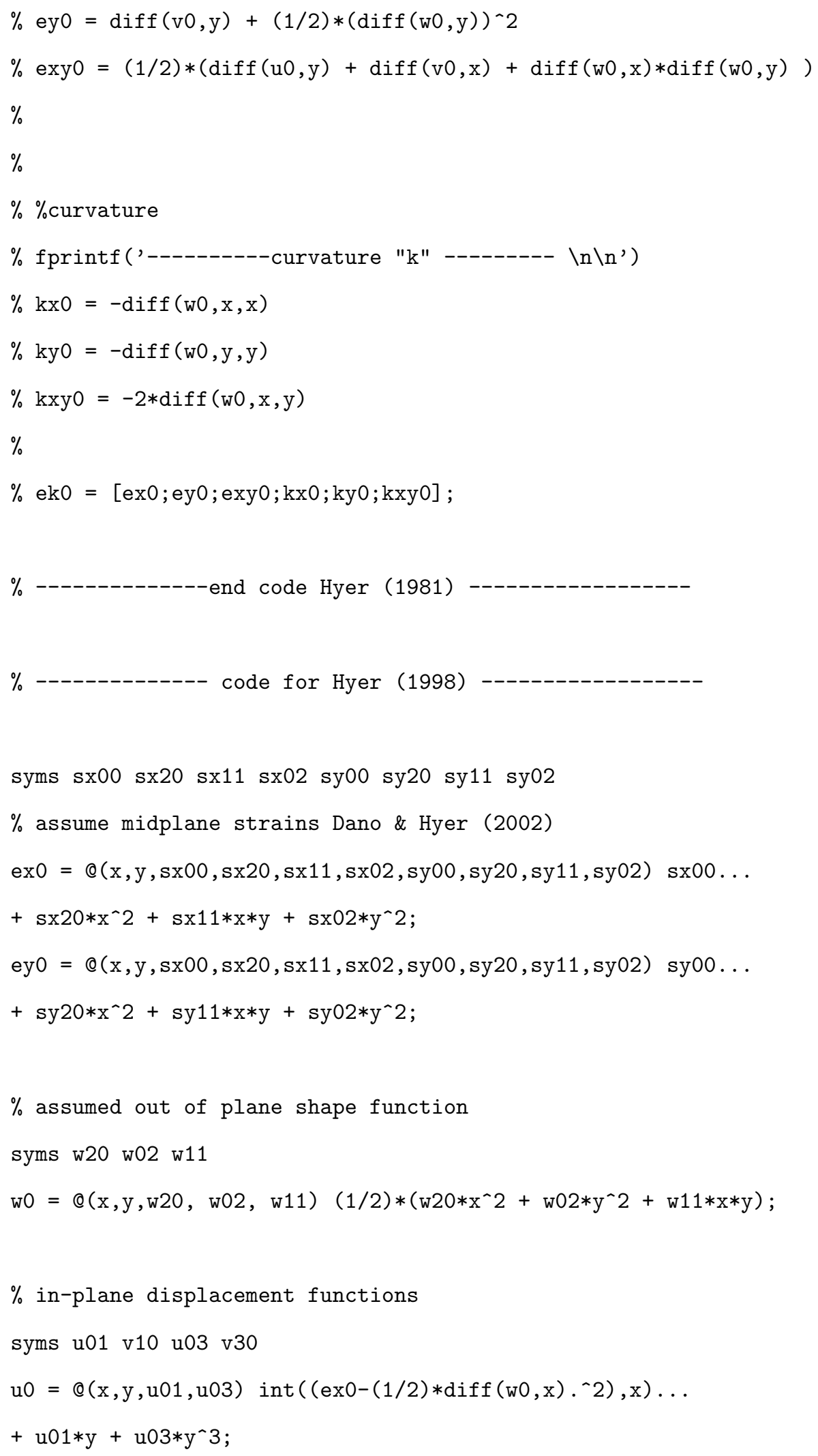




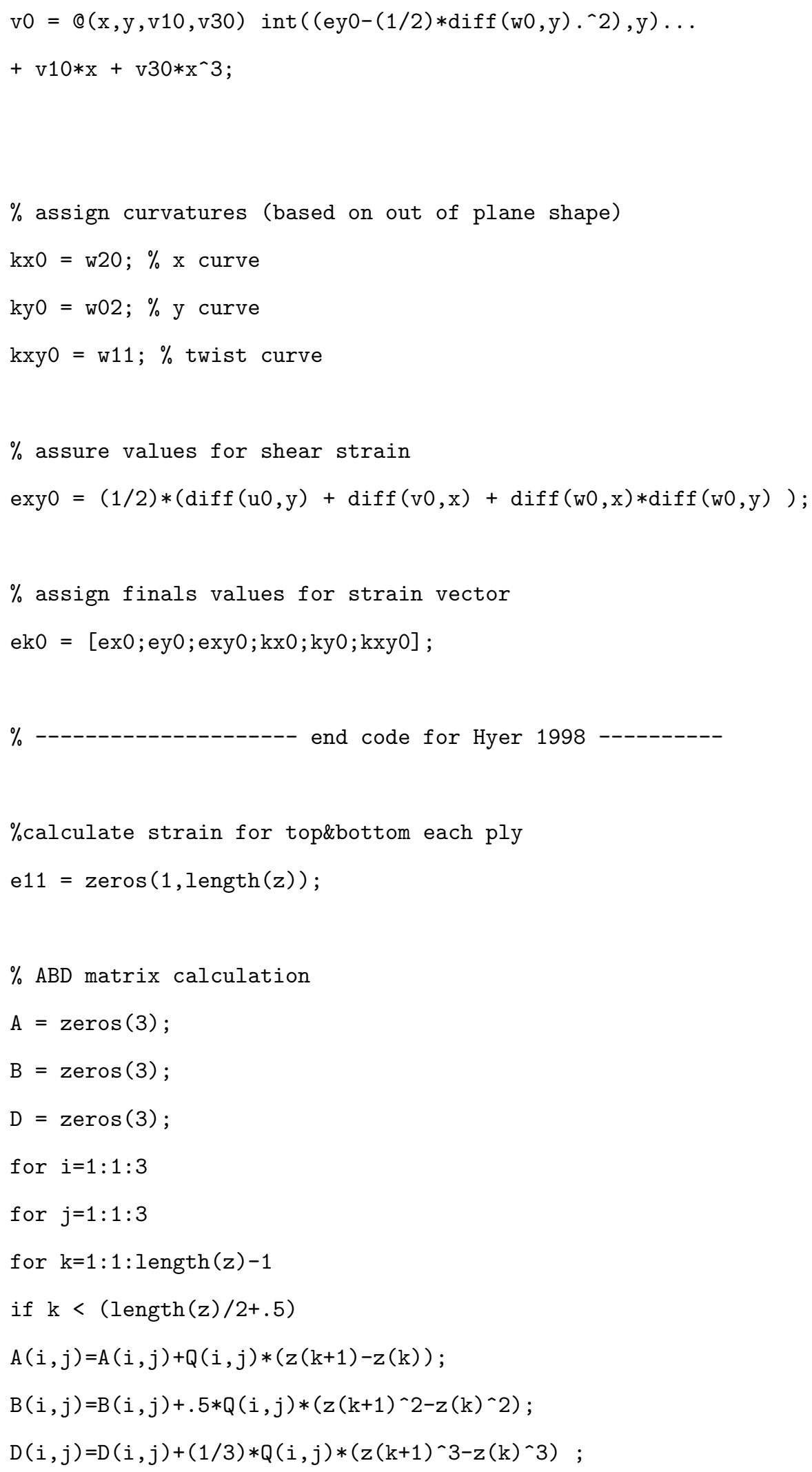




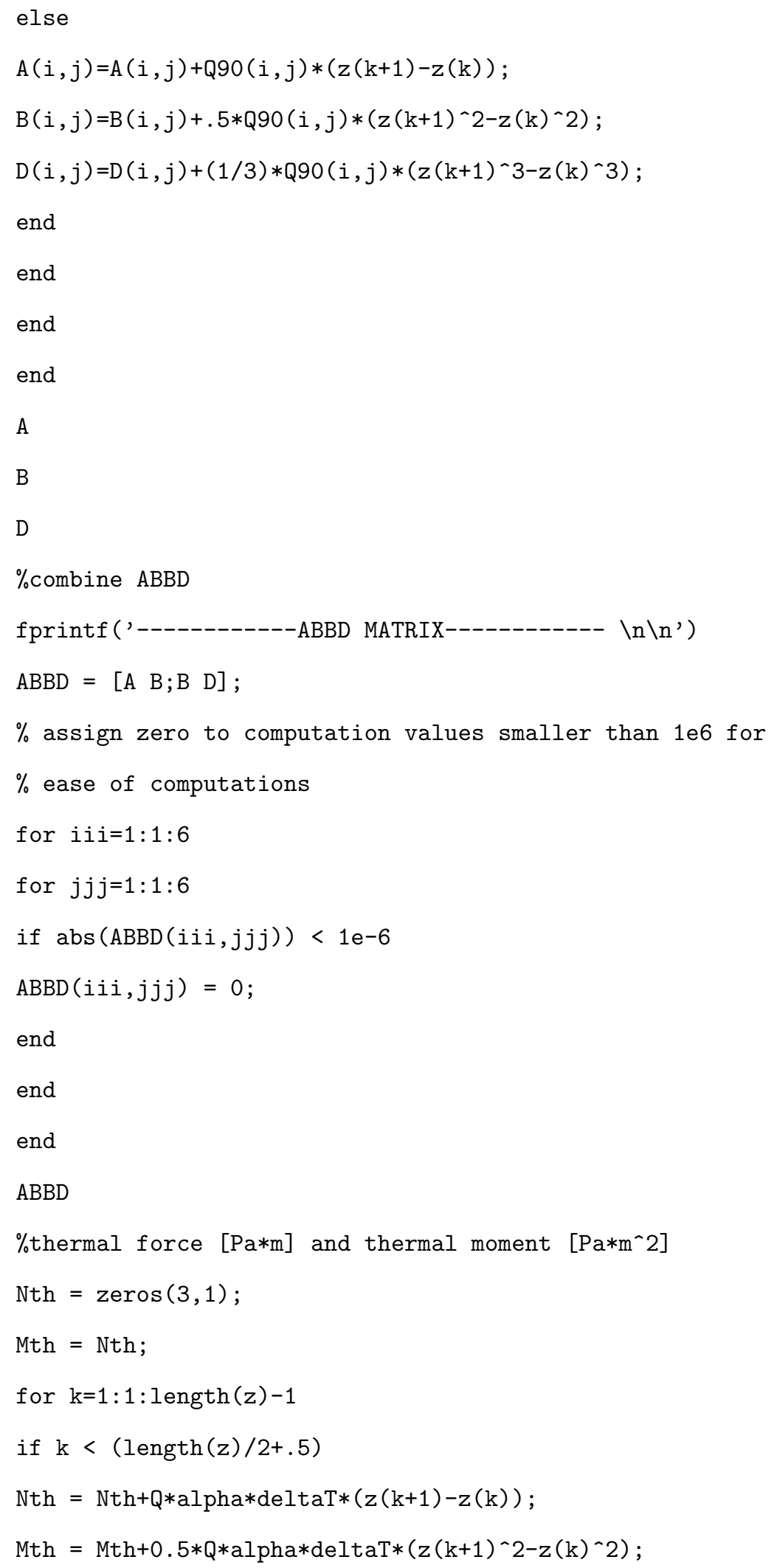




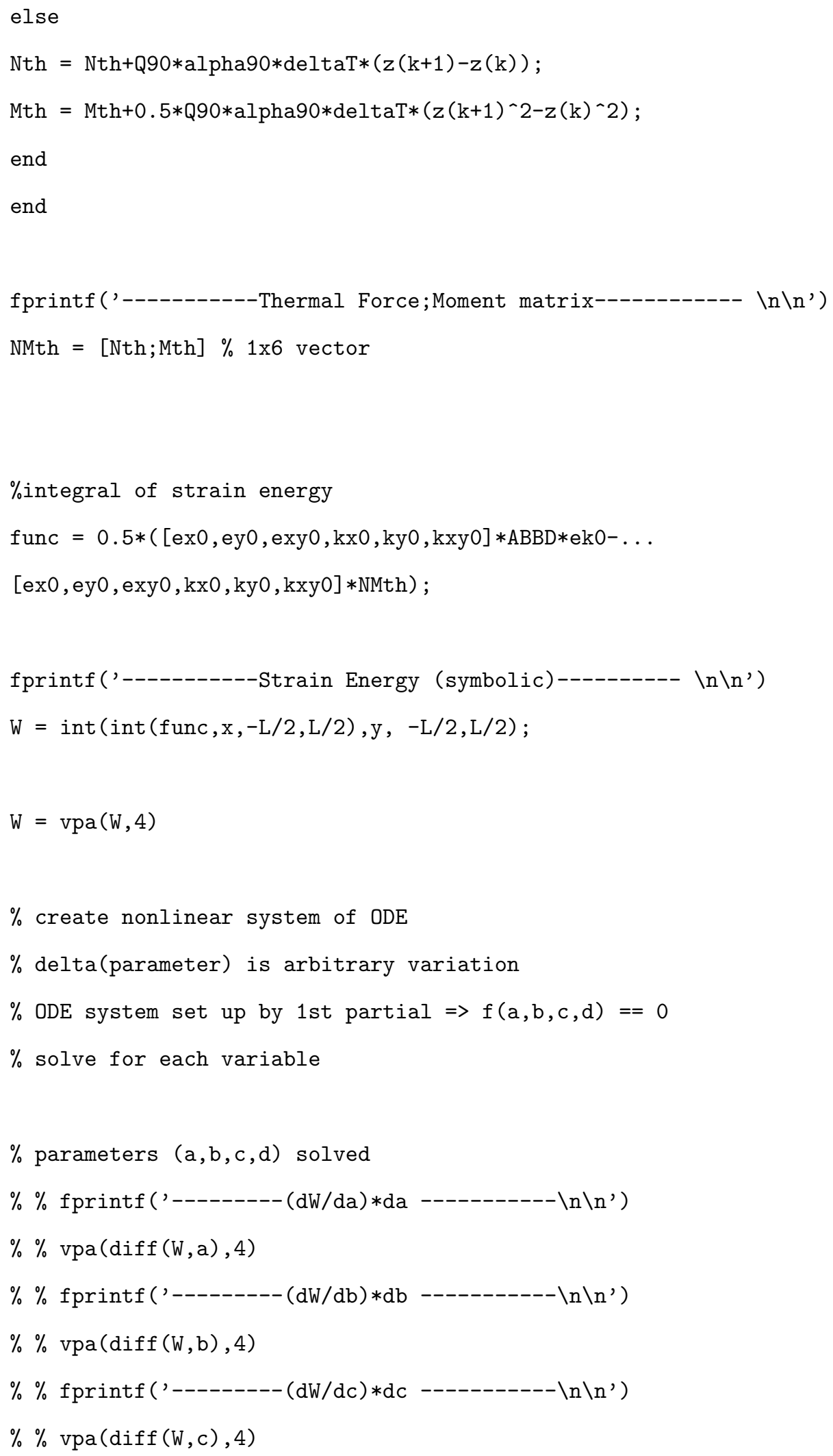




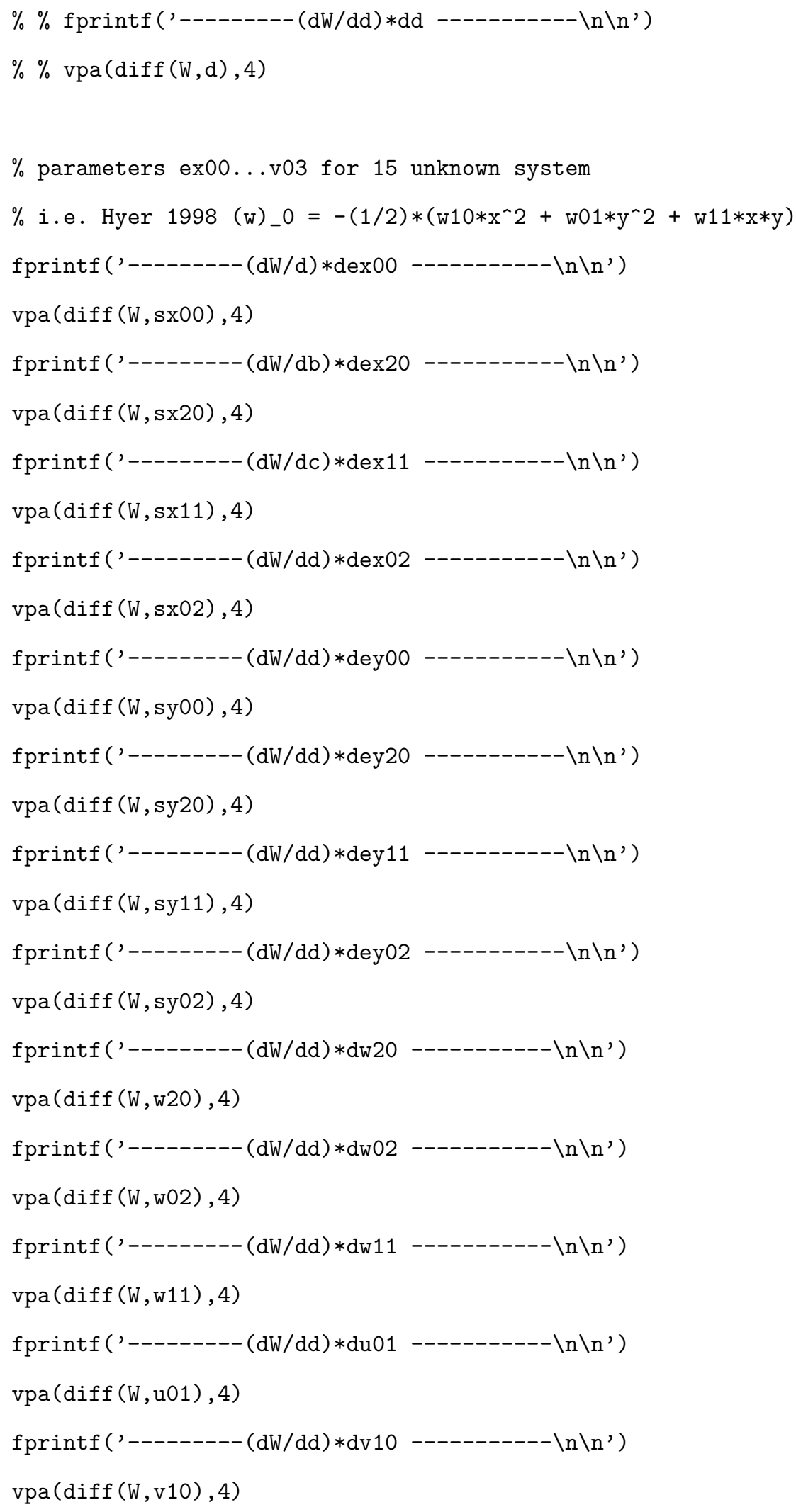




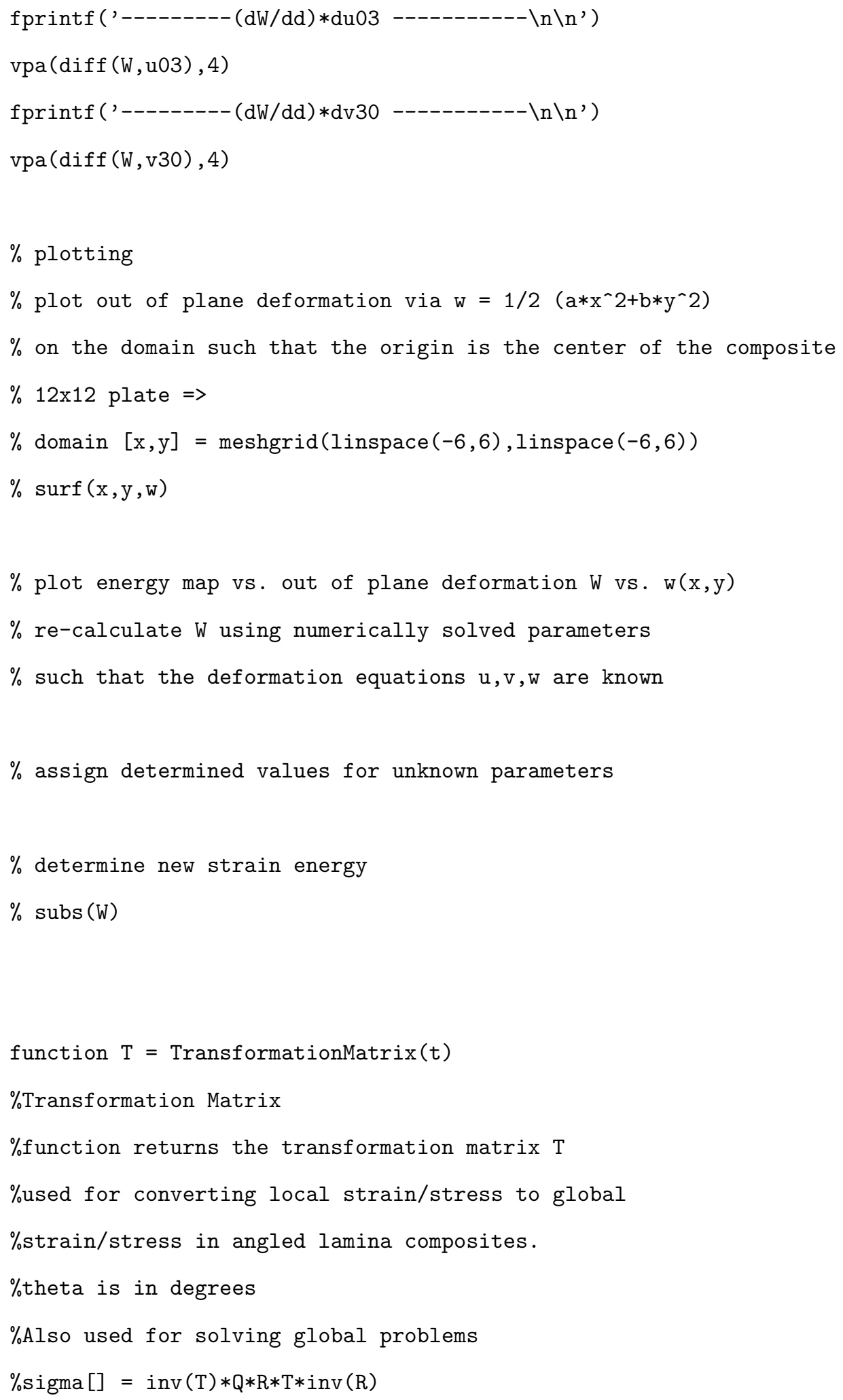




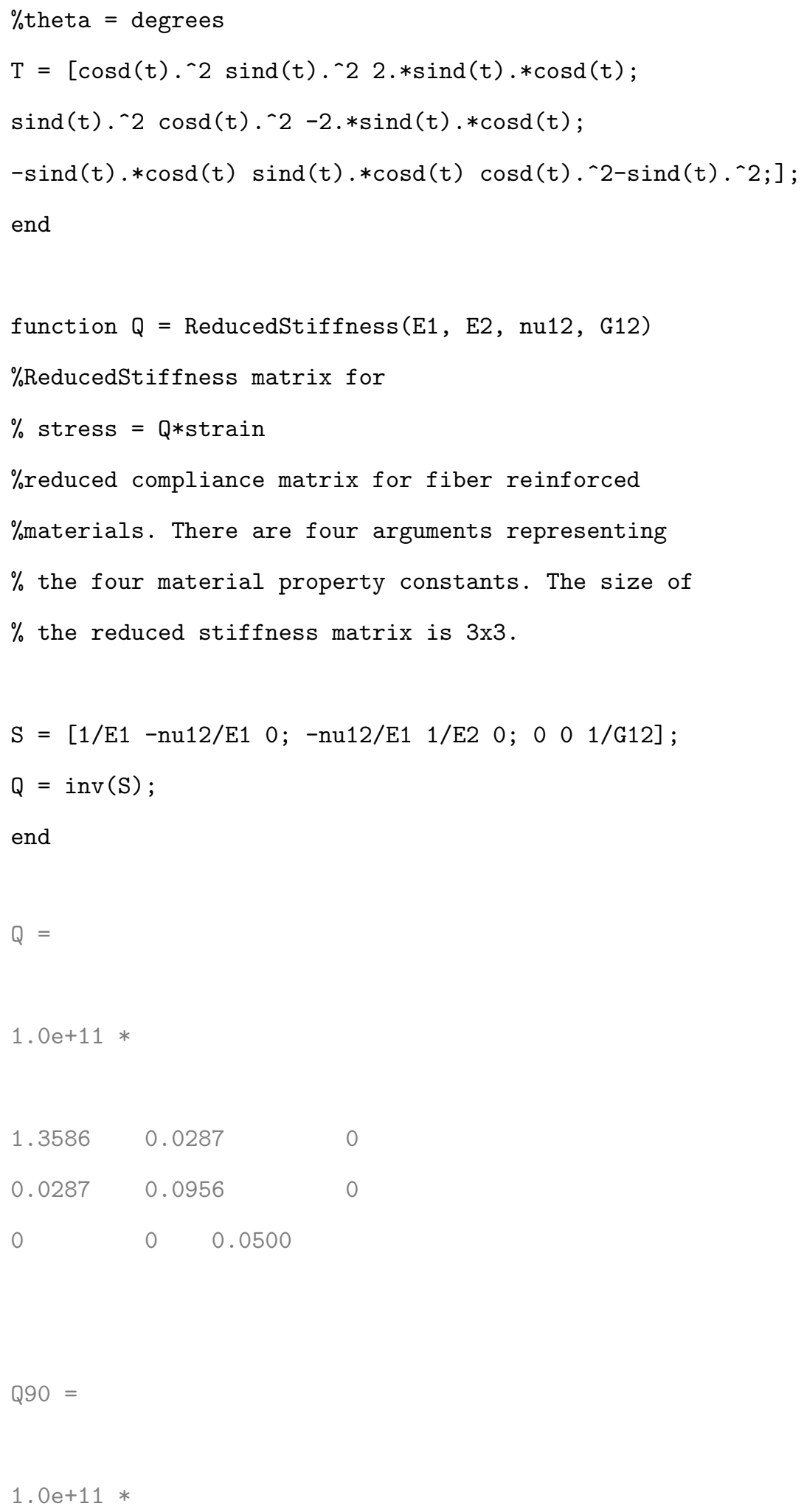




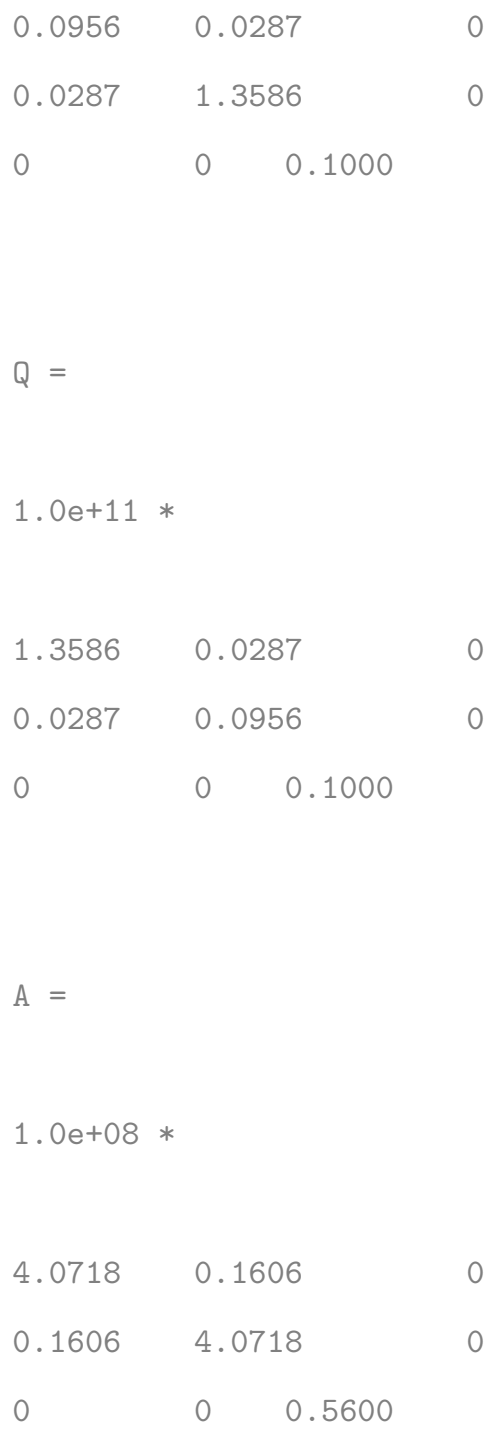




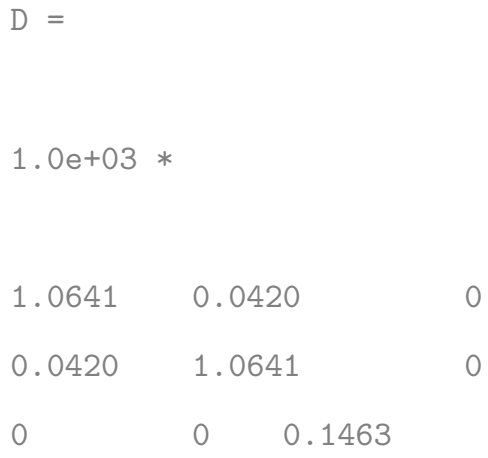

\begin{tabular}{|c|c|c|c|c|}
\hline 4.0718 & 0.1606 & 0 & -0.0050 & 0 \\
\hline 0.1606 & 4.0718 & 0 & 0 & 0.0050 \\
\hline
\end{tabular}

$\begin{array}{llllll}-0.0050 & 0 & 0 & 0.0000 & 0.0000 & 0\end{array}$

$\begin{array}{rrrrrr}0 & 0.0050 & 0 & 0.0000 & 0.0000 & 0 \\ 0 & 0 & 0 & 0 & 0 & 0.0000\end{array}$

---------- Thermal Force;Moment matrix------------

NMth $=$

$1.0 e+05 *$

$-1.3749$ 
$-1.3749$

0

$-0.0010$

0.0010

0

Strain Energy (symbolic)

$\mathrm{W}=$

$1.701 \mathrm{e} 8 * \mathrm{sx} 00^{\sim} 2+2.368 \mathrm{e} 7 * \mathrm{sx} 00 * \mathrm{sx} 02+2.368 \mathrm{e} 7 * \mathrm{sx} 00 * \mathrm{sx} 20+$

$1.342 \mathrm{e} 7 * \mathrm{sx} 00 * \mathrm{sy} 00+9.341 \mathrm{e} 5 * \mathrm{sx} 00 * \mathrm{sy} 02+9.341 \mathrm{e} 5 * \mathrm{sx} 00 * \mathrm{sy} 20-$

$4.136 \mathrm{e} 5 * \mathrm{sx} 00 * \mathrm{w} 20+57433.0 * \mathrm{sx} 00+1.597 \mathrm{e} 6 * \mathrm{sx} 02 \sim 2+1.649 \mathrm{e} 6 * \mathrm{sx} 02 * \mathrm{~s} \times 20$

$+9.341 \mathrm{e} 5 * \mathrm{sx} 02 * \mathrm{sy} 00+1.171 \mathrm{e} 5 * \mathrm{sx} 02 * \mathrm{sy} 02+2.918 \mathrm{e} 5 * \mathrm{sx} 02 * \mathrm{sy} 20$

$+1.134 \mathrm{e} 5 * \mathrm{~s} x 02 * \mathrm{w} 02 * \mathrm{w} 20-28344.0 * \mathrm{~s} x 02 * \mathrm{w} 11^{\wedge} 2-28799.0 * \mathrm{sx} 02 * \mathrm{w} 20$

$+3998.0 * \mathrm{sx} 02+8.37 \mathrm{e} 5 * \mathrm{sx} 11^{\wedge} 2+79200.0 * \mathrm{sx} 11 * \mathrm{sy} 11$

$+4.071 \mathrm{e} 5 * \mathrm{sx} 11 * \mathrm{u} 01+85022.0 * \mathrm{sx} 11 * \mathrm{u} 03+4.071 \mathrm{e} 5 * \mathrm{sx} 11 * \mathrm{v} 10$

$+1.53 \mathrm{e} 5 * \mathrm{~s} \times 11 * \mathrm{v} 30+7085.0 * \mathrm{~s} \times 11 * \mathrm{w} 02 * \mathrm{w} 11$

$+12755.0 * \mathrm{sx} 11 * \mathrm{w} 11 * \mathrm{w} 20+1.484 \mathrm{e} 6 * \mathrm{~s} \times 20^{\sim} 2+9.341 \mathrm{e} 5 * \mathrm{~s} \times 20 * \mathrm{sy} 00$

$+65033.0 * \mathrm{~s} x 20 * \mathrm{sy} 02+1.171 \mathrm{e} 5 * \mathrm{~s} x 20 * \mathrm{sy} 20-28799.0 * \mathrm{~s} x 20 * \mathrm{w} 20$

$+3998.0 * \mathrm{sx} 20+1.701 \mathrm{e} 8 * \mathrm{sy} 00^{\sim} 2+2.368 \mathrm{e} 7 * \mathrm{sy} 00 * \mathrm{sy} 02$

$+2.368 \mathrm{e} 7 * \mathrm{sy} 00 * \mathrm{sy} 20+4.136 \mathrm{e} 5 * \mathrm{sy} 00 *$ w0 2

$+57433.0 *$ sy00 +1.484 e6 $*$ sy02^ $2+1.649$ e6 6 sy0 $2 *$ sy 20

$+28799.0 *$ sy0 $2 * w 02+3998.0 *$ sy02 +8.37 e5*sy11^2

$+4.071 \mathrm{e} 5 * \mathrm{sy} 11 * \mathrm{u} 01+1.53 \mathrm{e} 5 * \mathrm{sy} 11 * \mathrm{u} 03+4.071 \mathrm{e} 5 * \mathrm{sy} 11 * \mathrm{v} 10$

$+85022.0 * \mathrm{sy} 11 * \mathrm{v} 30+12755.0 * \mathrm{sy} 11 * \mathrm{w} 02 * \mathrm{w} 11$

$+7085.0 * \operatorname{sy} 11 * \mathrm{w} 11 * \mathrm{w} 20+1.597 \mathrm{e} 6 * \mathrm{sy} 20^{\wedge} 2+1.134 \mathrm{e} 5 * \mathrm{sy} 20 * \mathrm{w} 02 * \mathrm{w} 20$

$+28799.0 * \mathrm{sy} 20 * \mathrm{w} 02-28344.0 * \mathrm{sy} 20 * w 11^{\wedge} 2+3998.0 * \mathrm{sy} 20+5.848 \mathrm{e} 6 * \mathrm{u} 01^{\wedge} 2$

$+2.443 \mathrm{e} 6 * \mathrm{u} 01 * \mathrm{u} 03+1.17 \mathrm{e} 7 * \mathrm{u} 01 * \mathrm{v} 10+2.443 \mathrm{e} 6 * \mathrm{u} 01 * \mathrm{v} 30+2.036 \mathrm{e} 5 * \mathrm{u} 01 * \mathrm{w} 02 * \mathrm{w} 11$

$+2.036 \mathrm{e} 5 * \mathrm{u} 01 * \mathrm{w} 11 * \mathrm{w} 20+4.591 \mathrm{e} 5 * \mathrm{u} 03^{\wedge} 2+2.443 \mathrm{e} 6 * \mathrm{u} 03 * \mathrm{v} 10+5.101 \mathrm{e} 5 * \mathrm{u} 03 * \mathrm{v} 30$ 


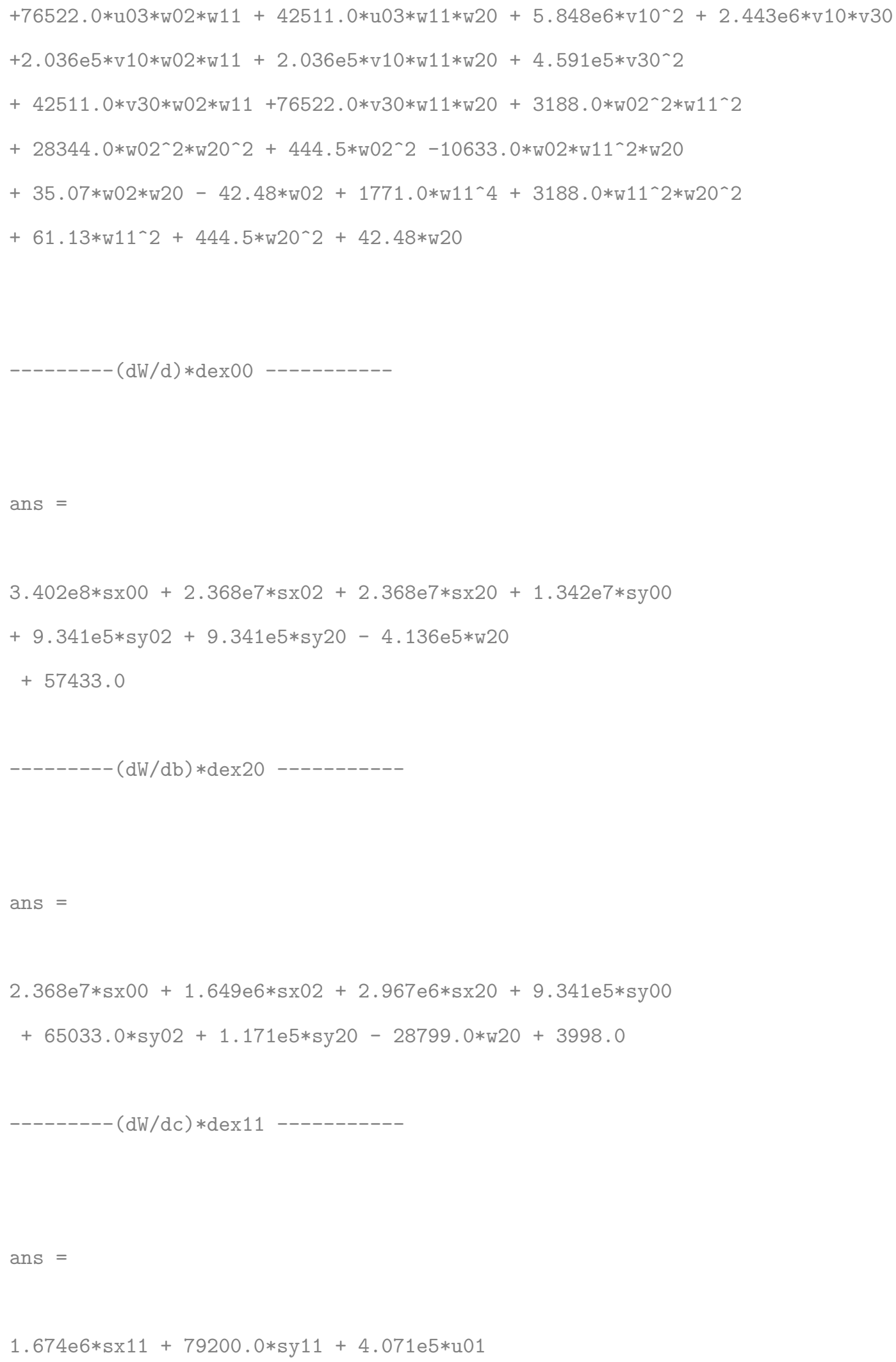




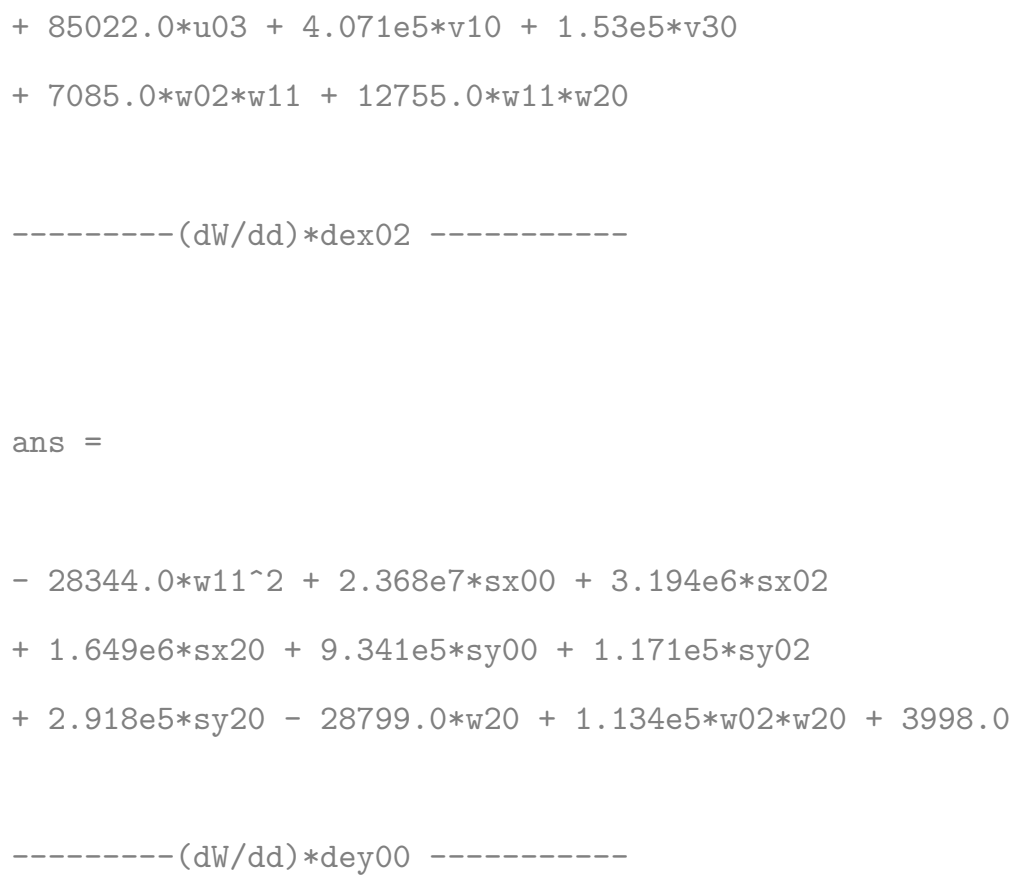




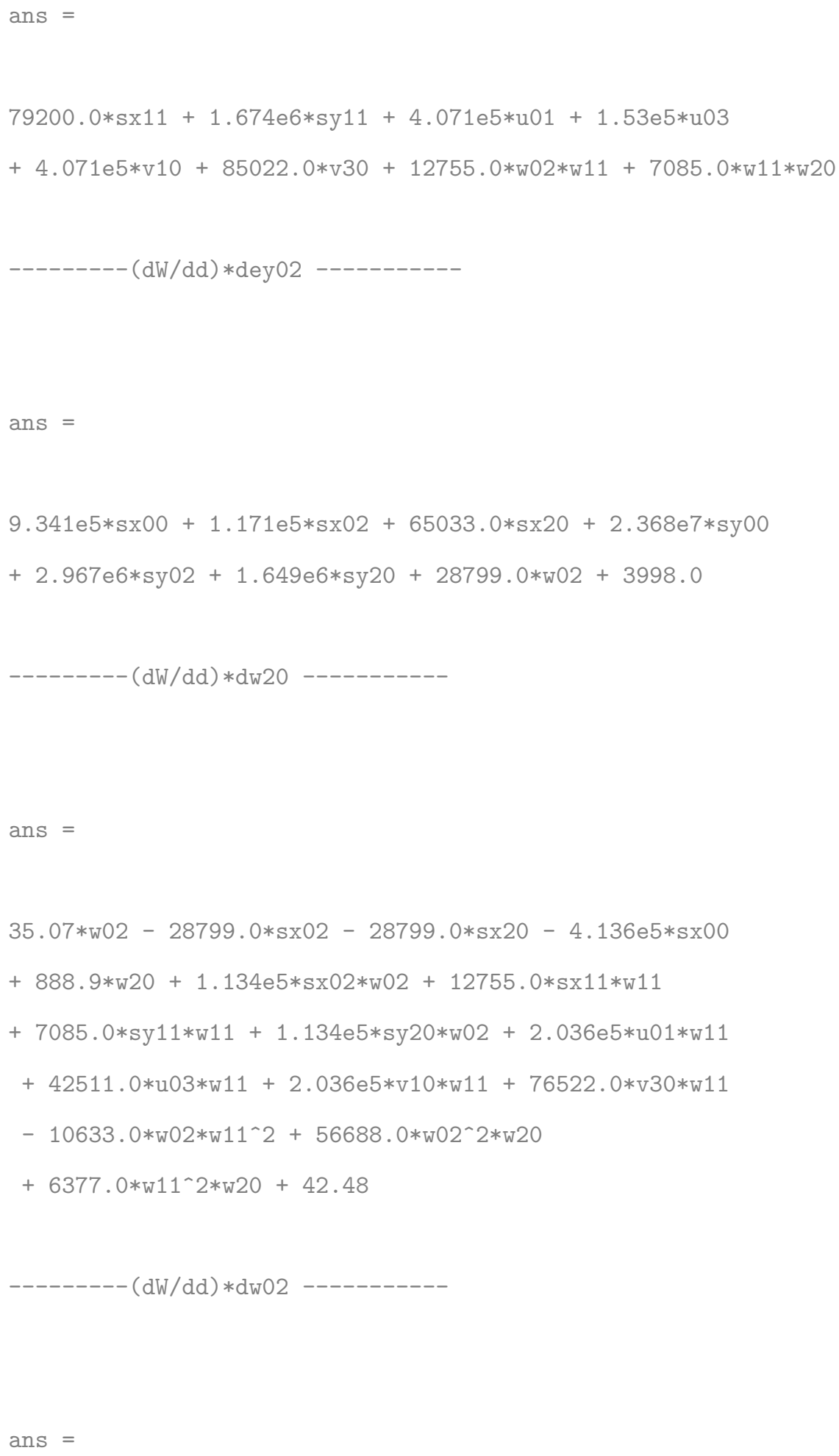




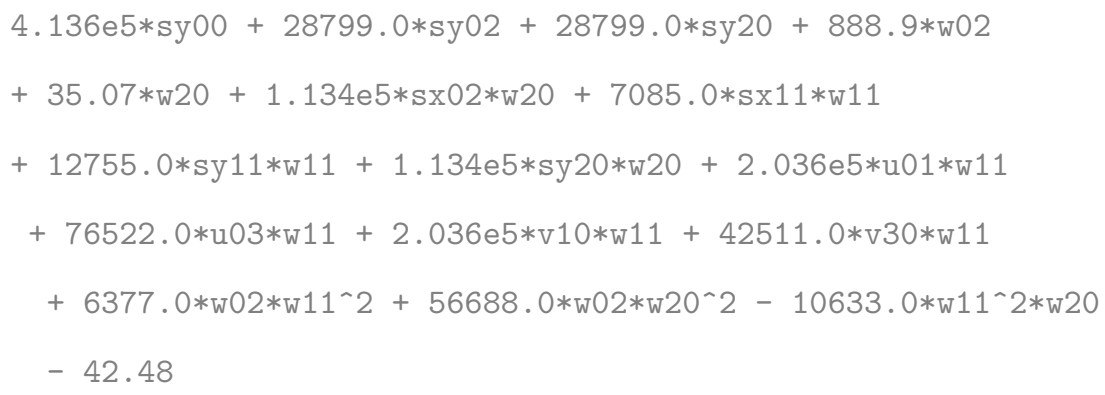




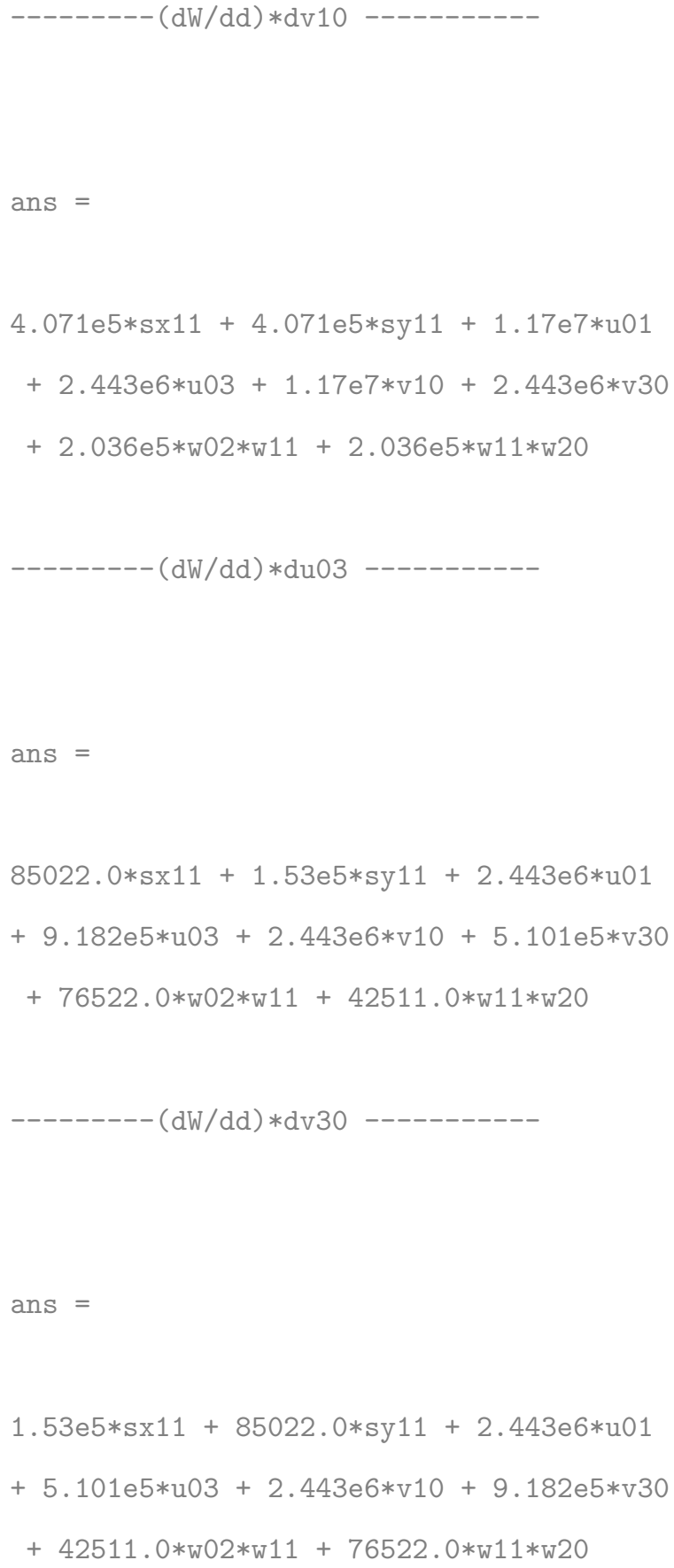

\section{Bestfit Equation Script and Mean Displacements and Curvatures}




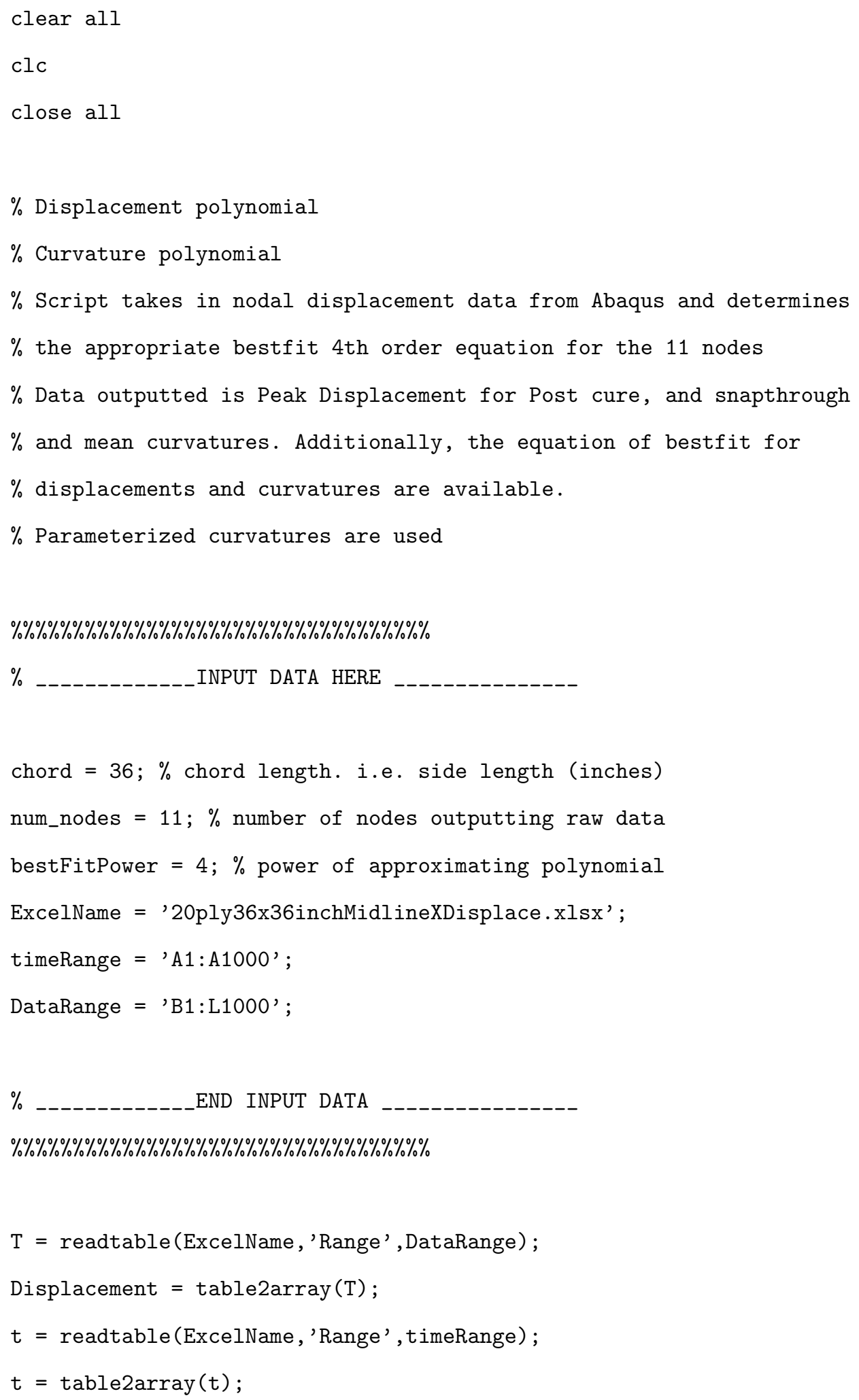




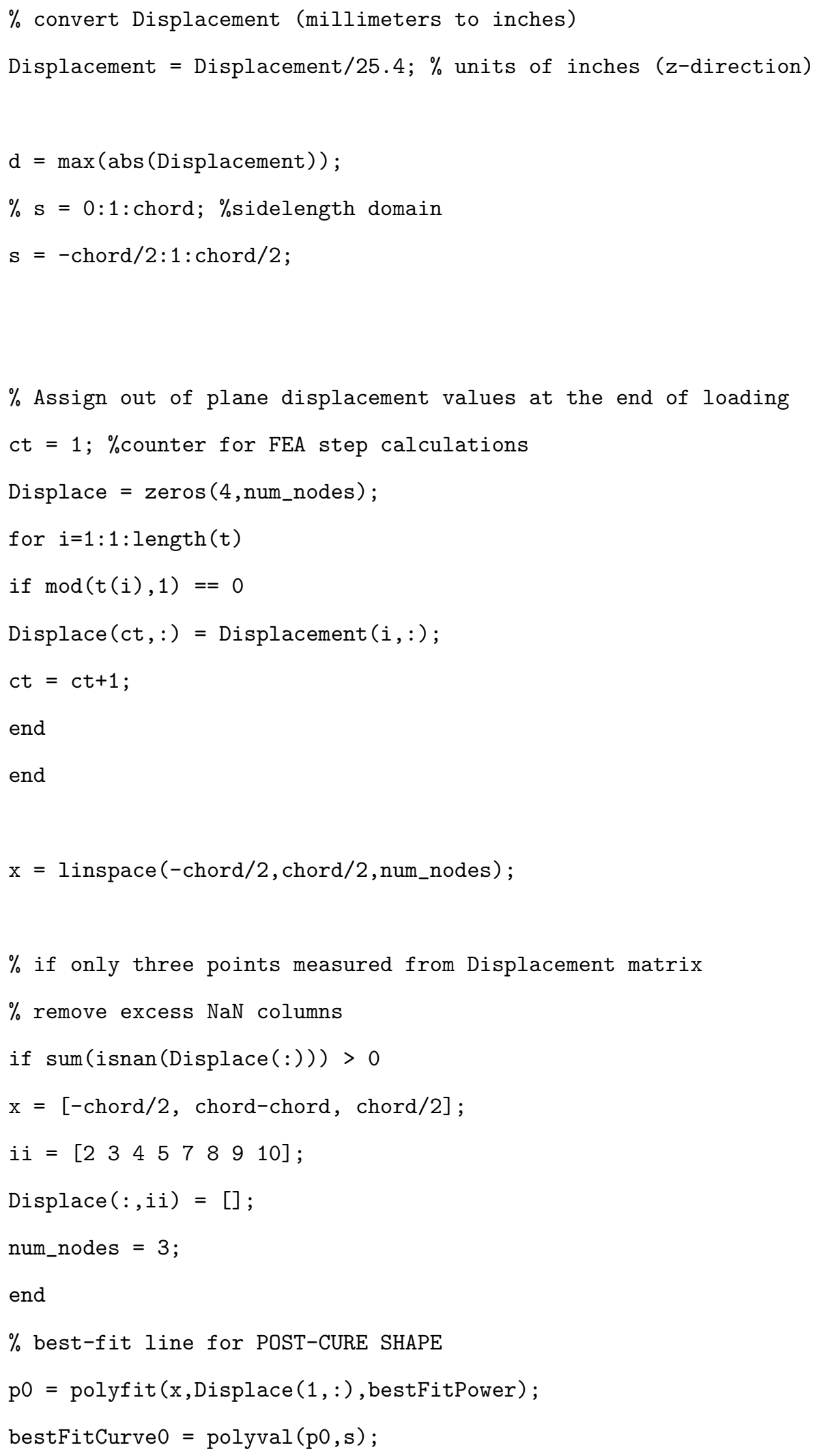




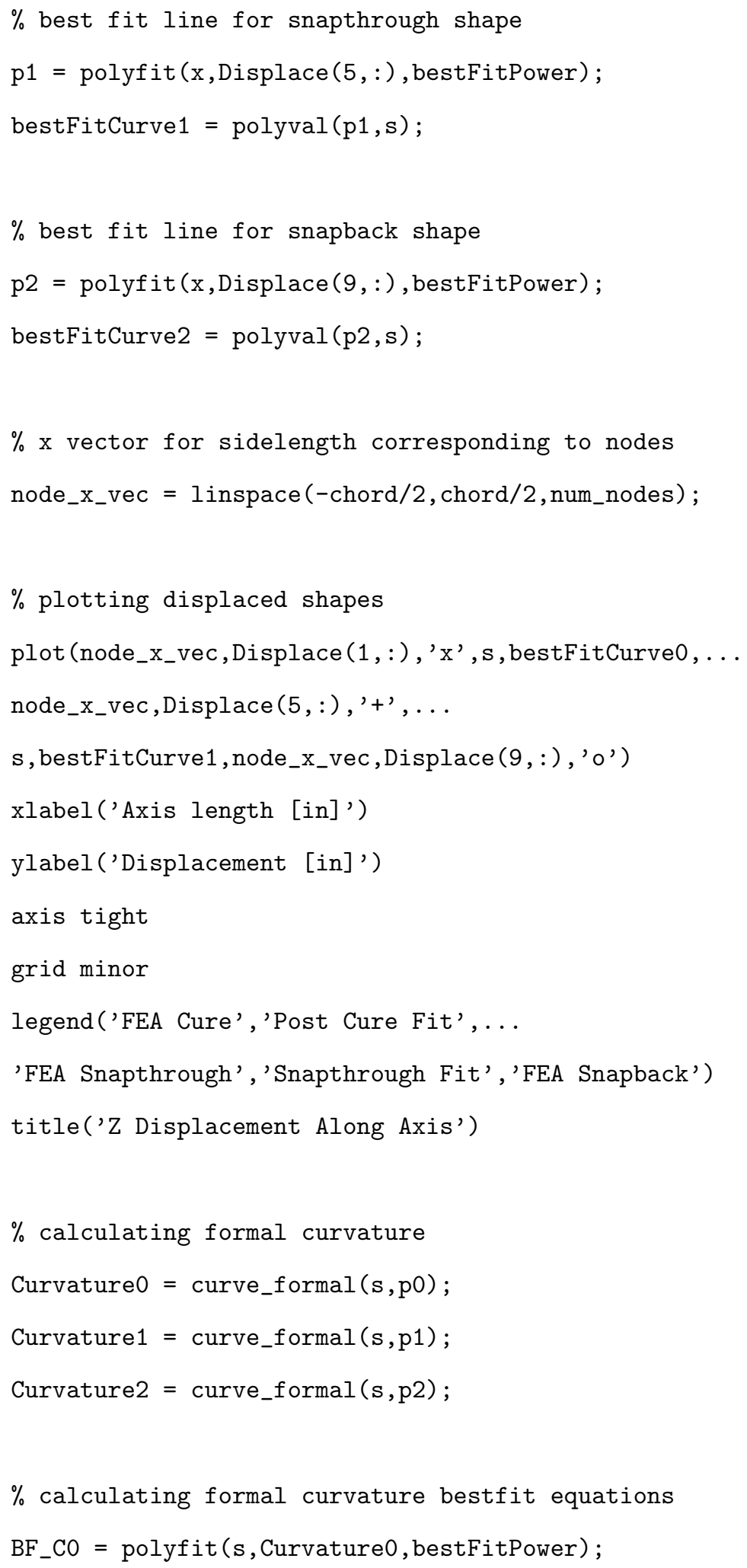




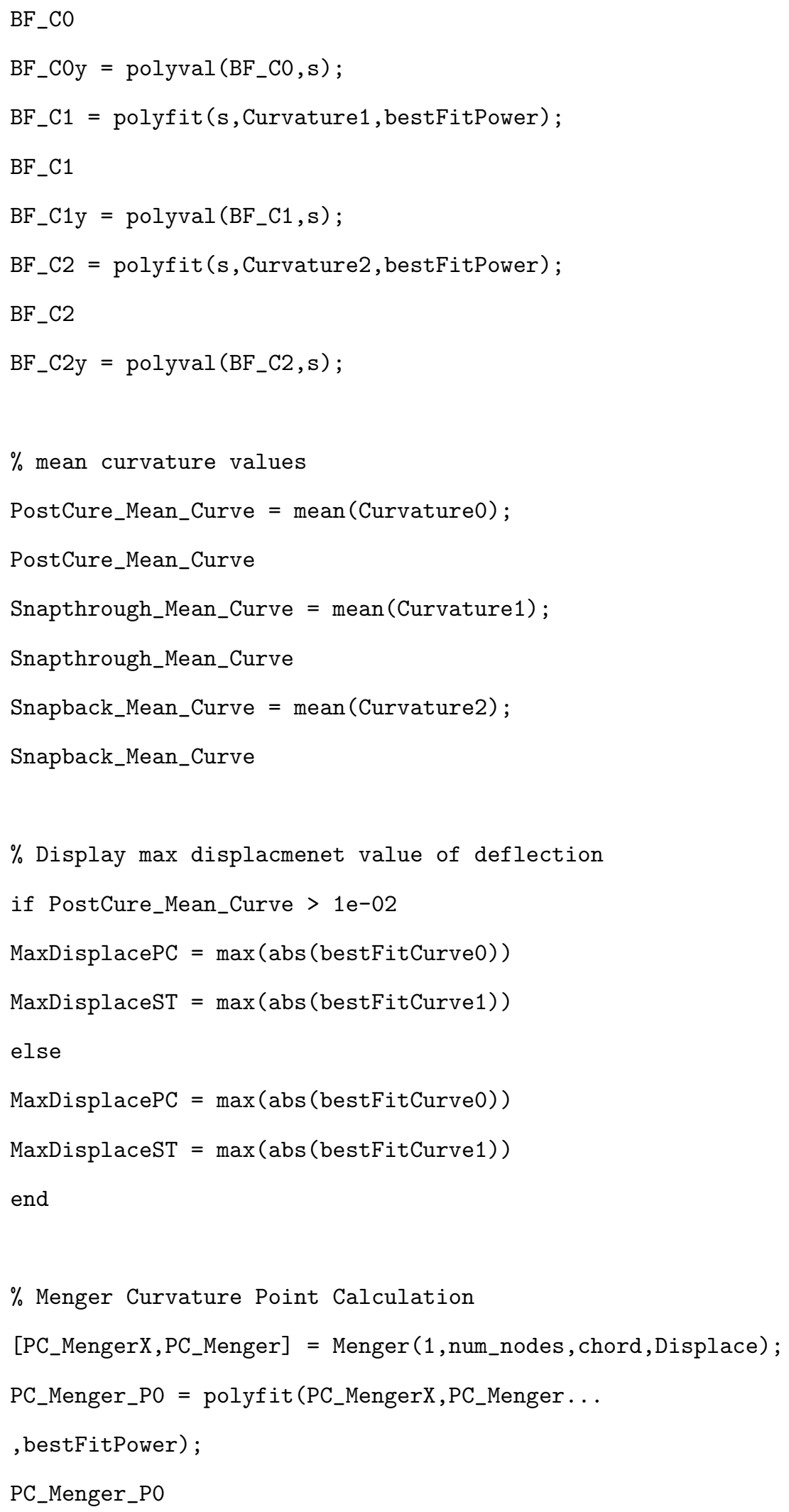


[ST_MengerX,ST_Menger] = Menger (5,num_nodes, chord,Displace);

ST_Menger_P1 = polyfit(ST_MengerX,ST_Menger, bestFitPower);

ST_Menger_P1

[SB_MengerX,SB_Menger] = Menger (9, num_nodes, chord,Displace);

SB_Menger_P2 = polyfit(SB_MengerX,SB_Menger, bestFitPower);

$\%$ Menger Bestfit calculation

MengerPO_BFy = polyval (PC_Menger_PO,s);

MengerP1_BFy = polyval (ST_Menger_P1, s);

MengerP2_BFy = polyval (SB_Menger_P2,s);

$\%$ plotting Curvature vs. Sidelength

figure

subplot $\left(3,2,\left[\begin{array}{ll}1 & 2\end{array}\right]\right) \%$ FIGURE 2

plot (s, Curvature0, ' . ', PC_MengerX, PC_Menger , 'o ' , . . .

s, BF_COy, s, MengerPo_BFy)

legend('Formal', 'Menger', 'Formal BF', 'Menger BF')

ylabel ('Curvature [1/in]')

title('Post Cure Curvature Along Axis')

axis tight

grid minor

hold on

$\operatorname{subplot}\left(3,2,\left[\begin{array}{ll}3 & 4\end{array}\right]\right)$

plot (s, Curvature1, '. ', ST_MengerX, ST_Menger, 'o' , . . .

s , BF_C1y, s, MengerP1_BFy)

ylabel ('Curvature [1/in]')

title('Snapthrough Curvature Along Axis')

axis tight

grid minor

legend('Formal', 'Menger', 'Formal BF', 'Menger BF') 


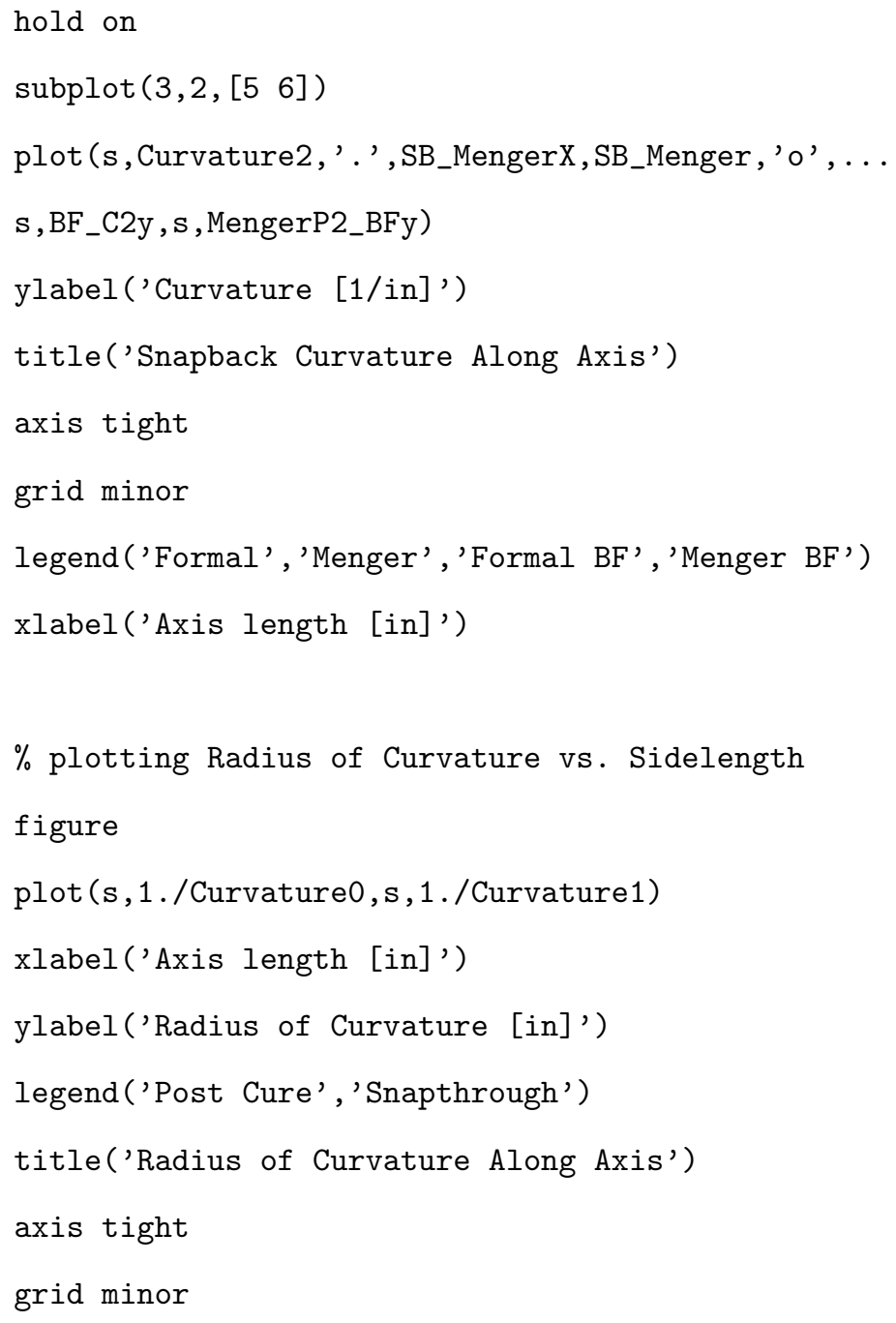




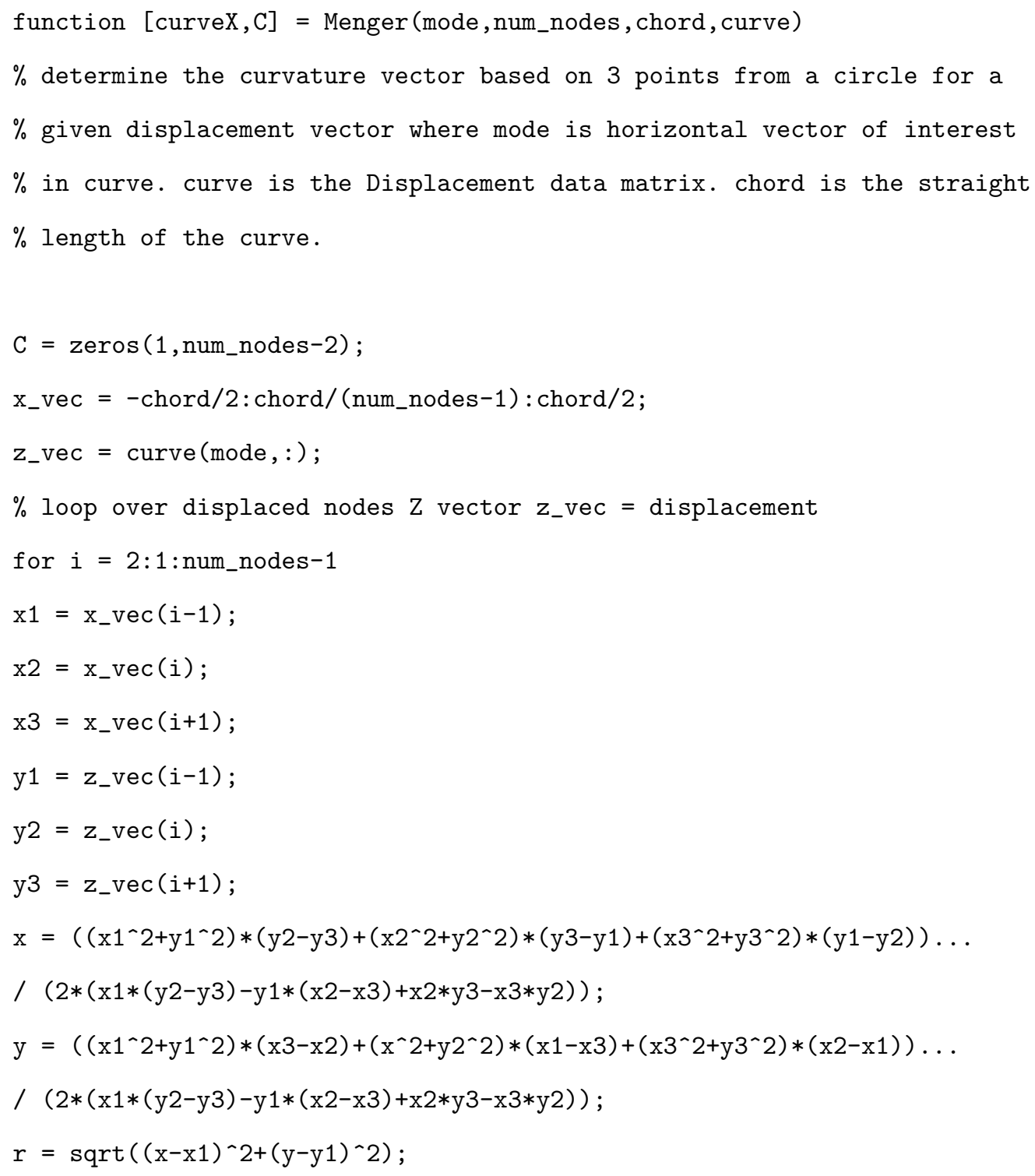




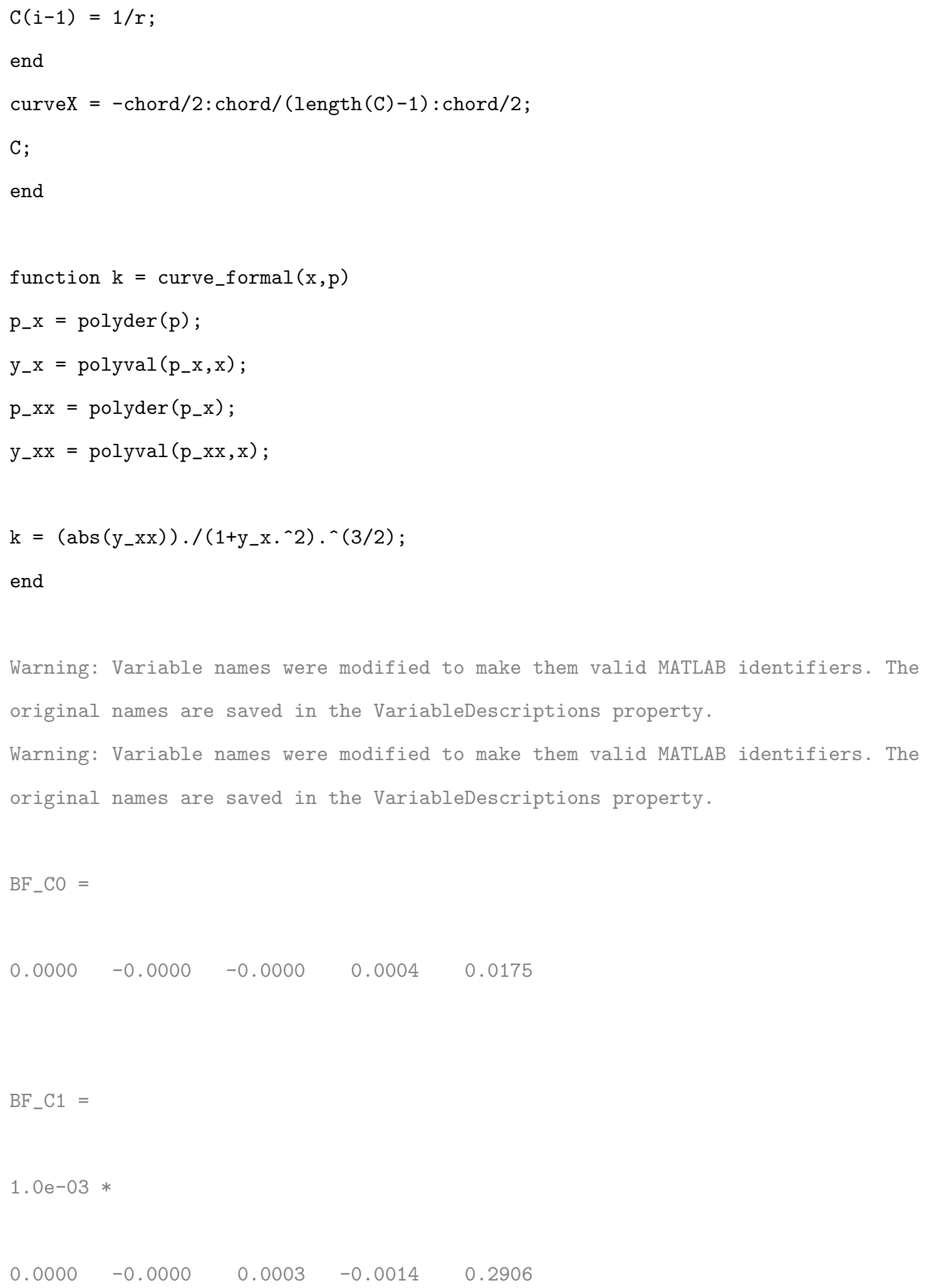




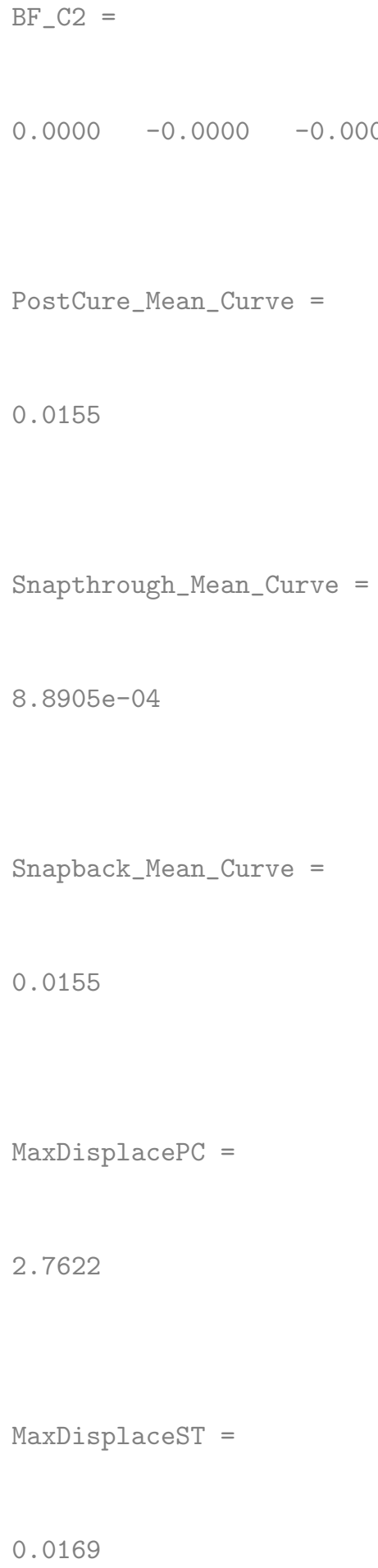


PC_Menger_PO =
0.0000
0.0000
$-0.0001$
$-0.0000$
0.0139

ST_Menger_P1 =

$1.0 e-03 *$

$-0.0044$

0.0026

0.1605

\section{Z Displacement Along Axis}

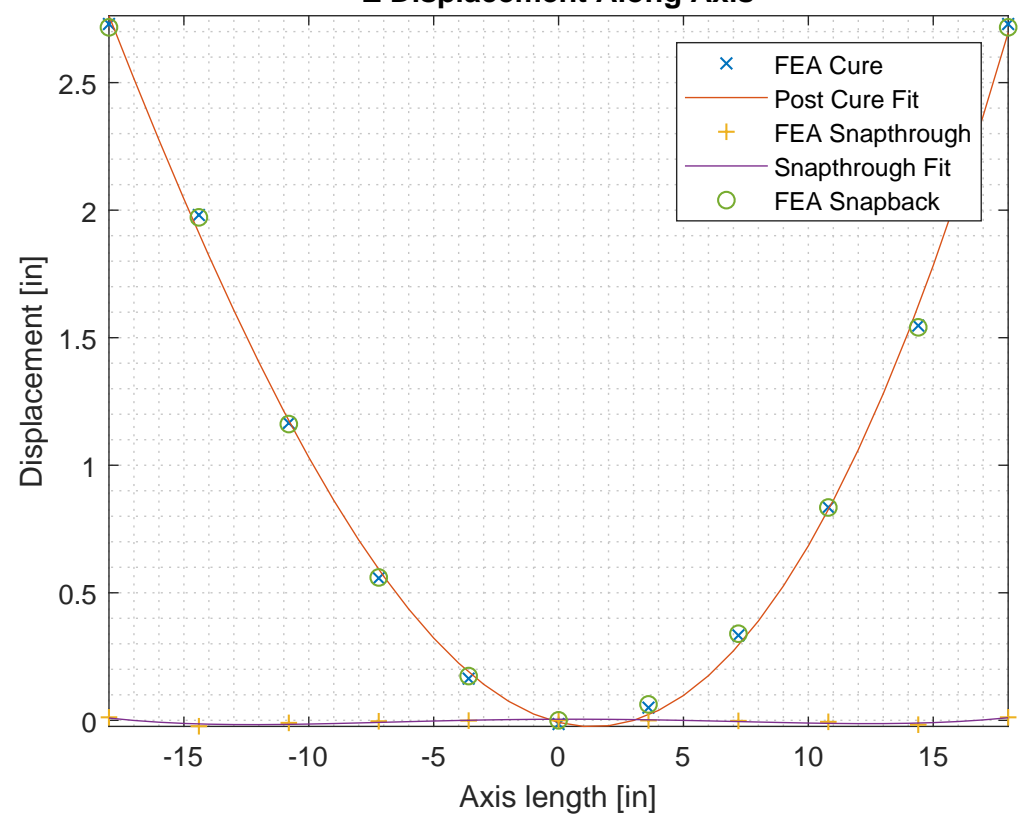



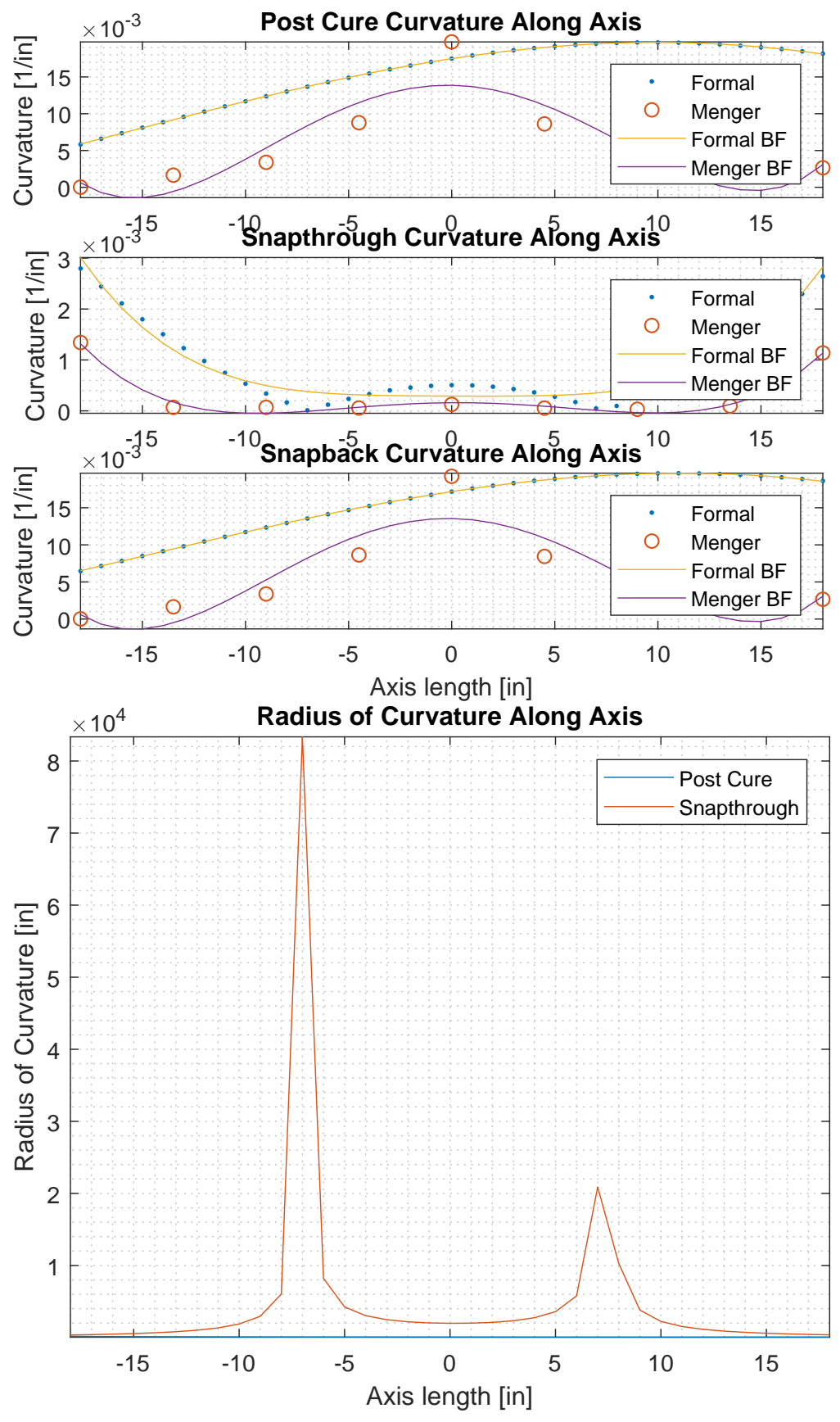

Bifurcation Plot Generation

clear all

close all

clc 
$\%$ goal of this script is to visualize the curvature polynomials

$\%$ for bistable laminates. The curvature polynomials are determined by

$\%$ fitting a polynomial function to the formal definition of curvature

$\%$ per point for a given domain. The formal definition of curvature

$\%$ is easily looked up. The formal definition of curvature is not the

$\%$ same as the Menger Curvature. The Menger Curvature is found from

$\%$ the equation of a circle that fits 3 points. Then the associated

$\%$ curvature is found based on the radii of said circle.

$\%$ It is desirable to nondimensionalize the curvature polynomials

$\%$ to see if the curvatures collapse to one master polynomial.

$\%$ The significance is to determine if curvature can be predicted

$\%$ from this "master polynomial."

$\%$ Additionally, this script will generate a bifurication plot

$\%$ and a nondimensionalized curvature vs. L/t bifuication plot

$\%$ and attempt to find a master polynomial

$\% \% \% \% \% \% \% \% \% \% \% \% \% \% \% \% \% \% \% \% \% \% \% \% \% \% \% \% \% \% \% \% \% \% \% \% \% \% \% \% \% \% \%$

$\%$ Input data

chord $=36 ; \%$ [inches]

thickness $=0.2 \mathrm{e}-3 / .0254 ; \%$ [inches $] 0.2 \mathrm{~mm}=0.016 "$ post-cure thickness

$\% \quad$ VALUES FROM EXCEL DATASHEET

ExcelName $=$ 'Curvature_Data.xlsx'; \% Imperial units

Coef_Curve_Poly = 'I3:M30'; \% Coefficients of bestfit Curvature

Length $=$ 'B4:B30'; \% Sidelength dimension laminate [inches]

num_ply = 'C4:C30'; \% num of plies

PC_mean_curve = 'G4:H30'; \% avg value curvature post cure

ST_mean_curve $=$ 'N4:030'; \%avg value curvature post cure 


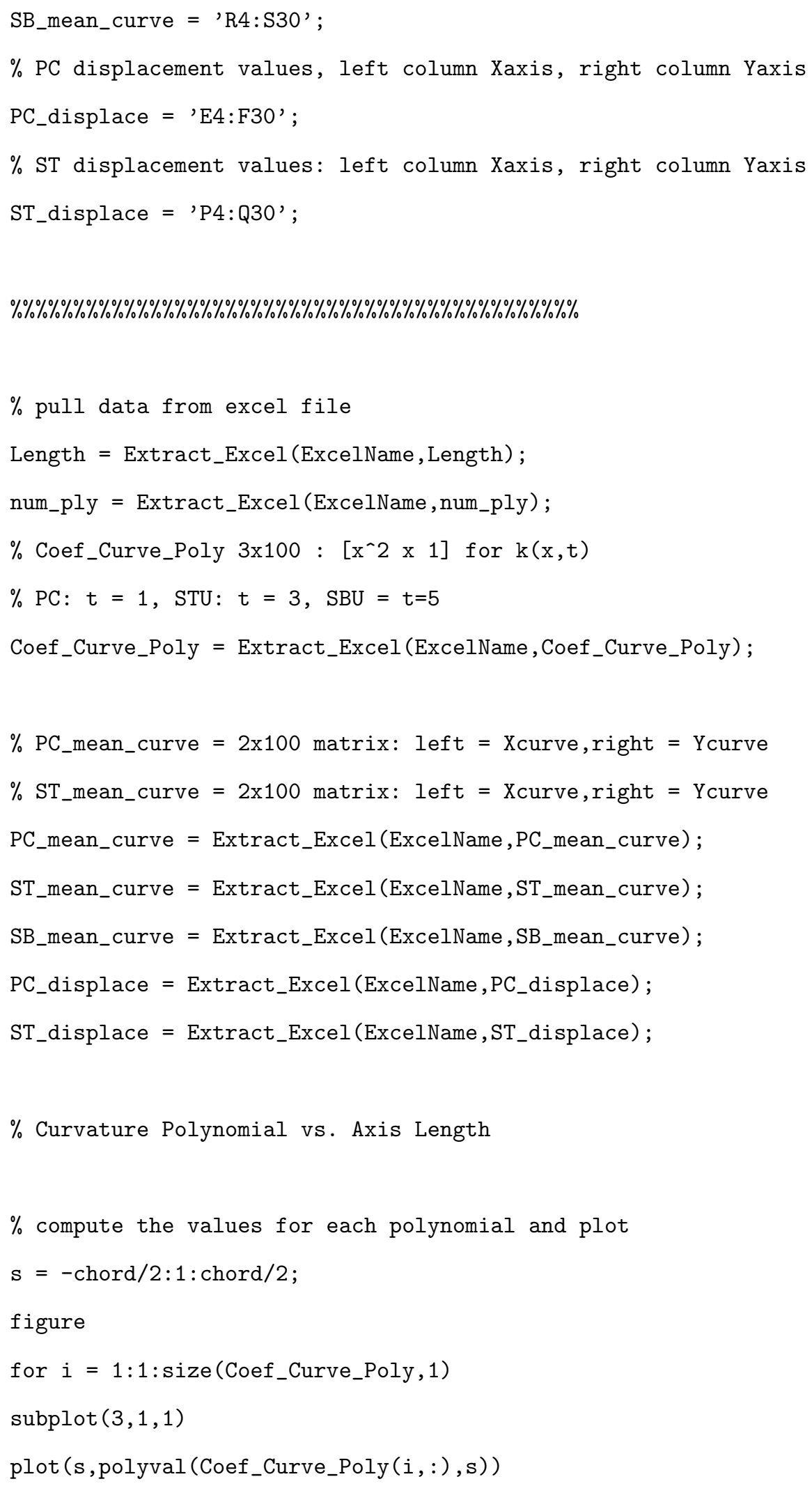




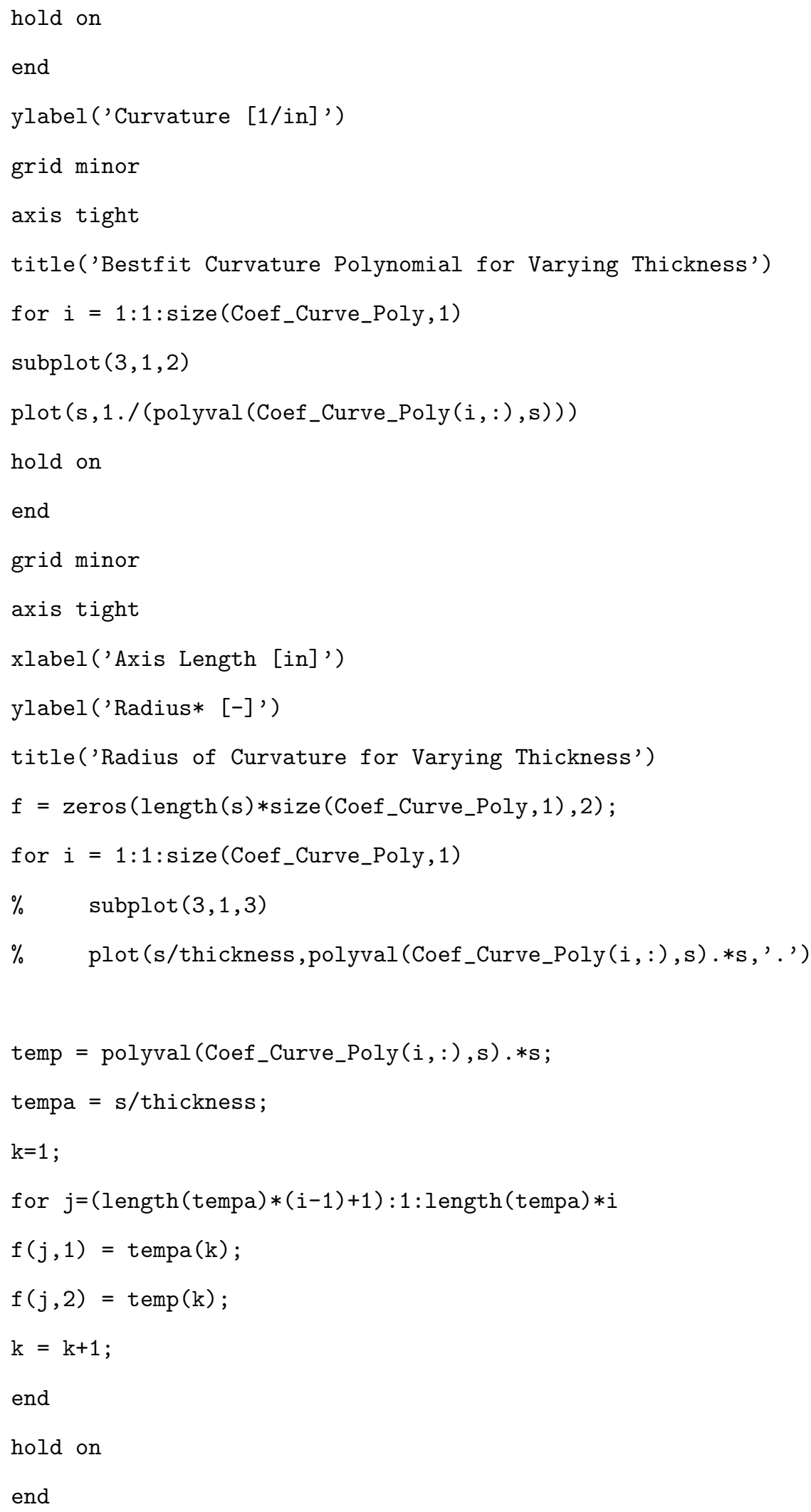




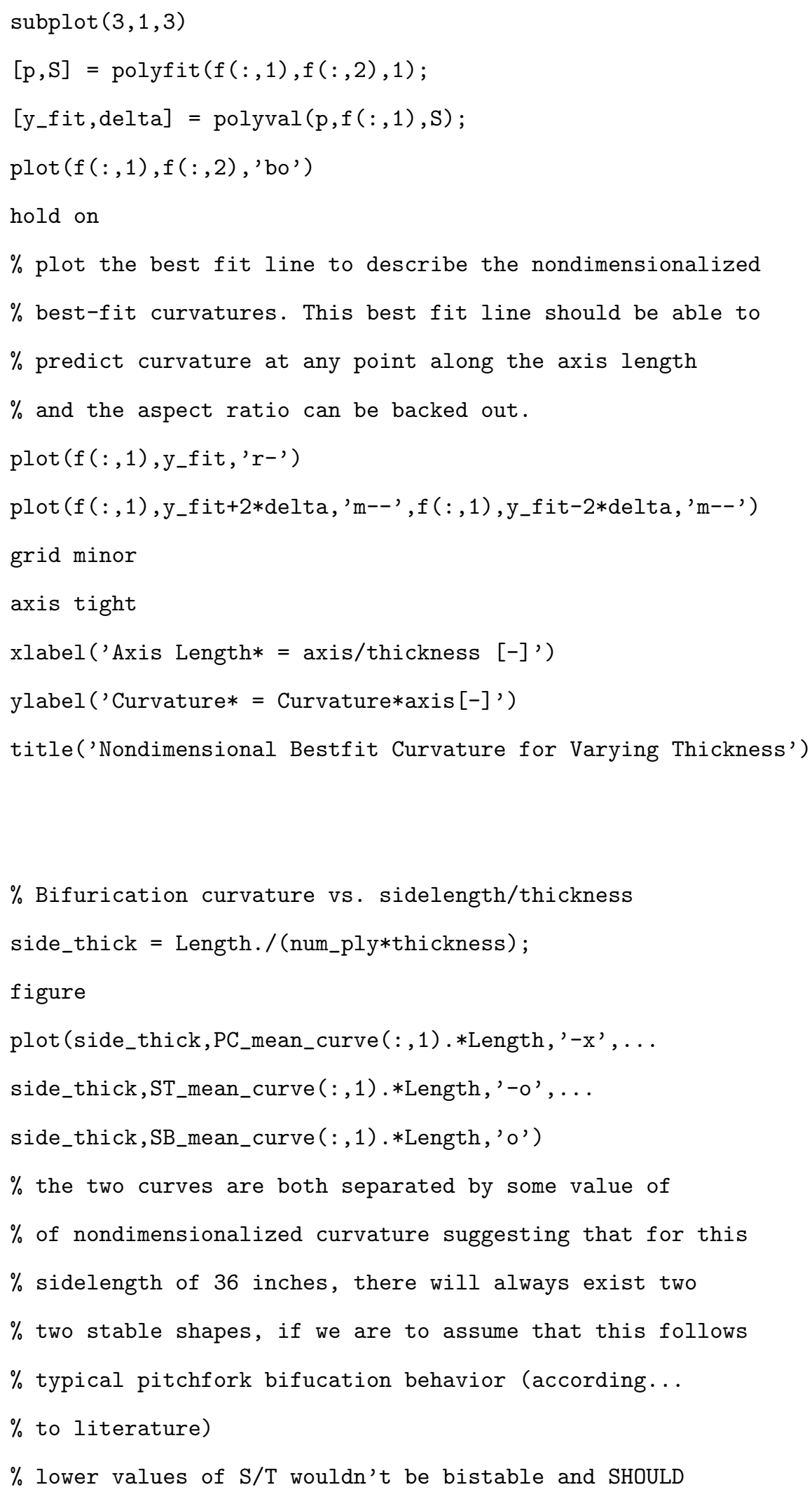




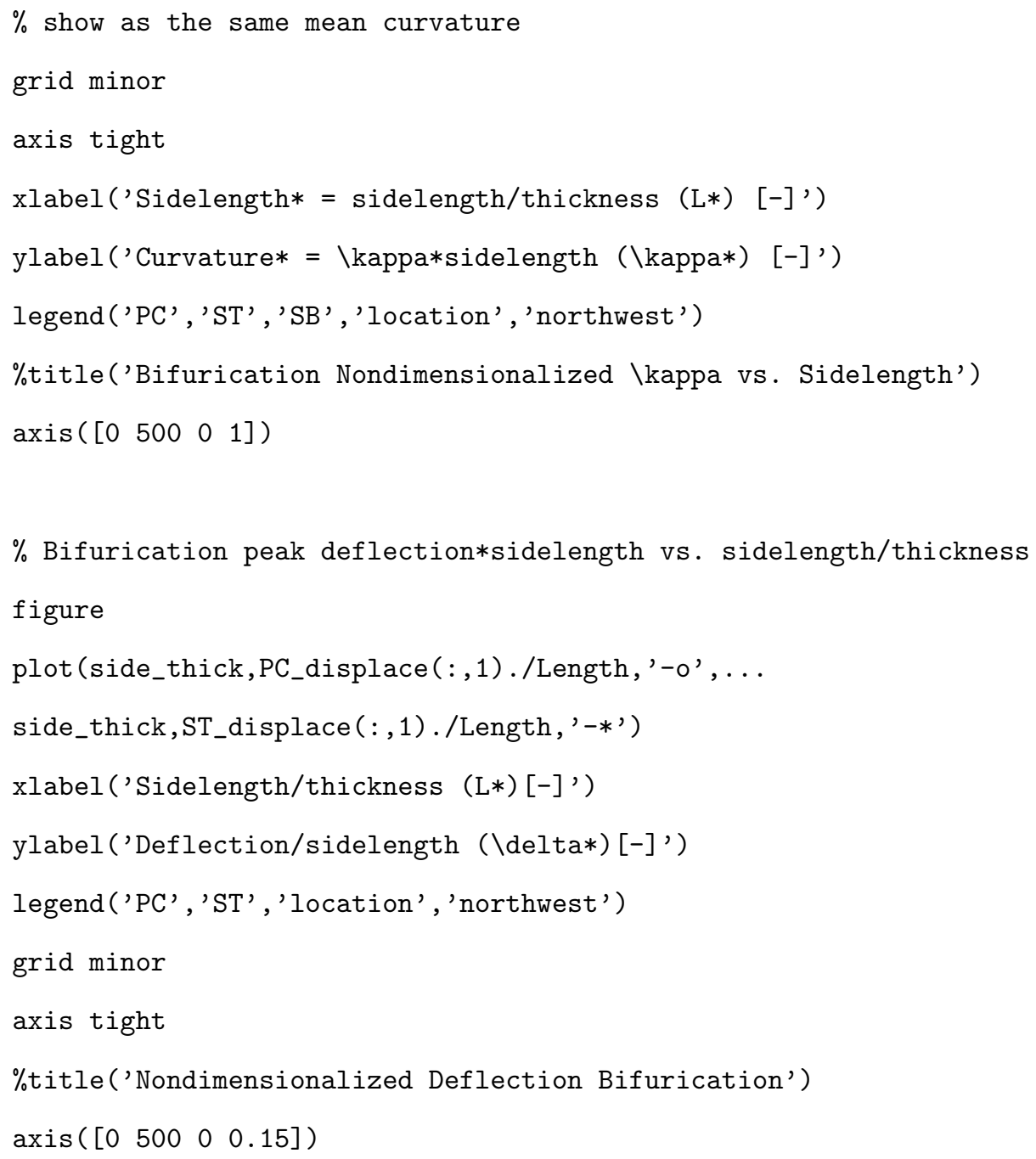



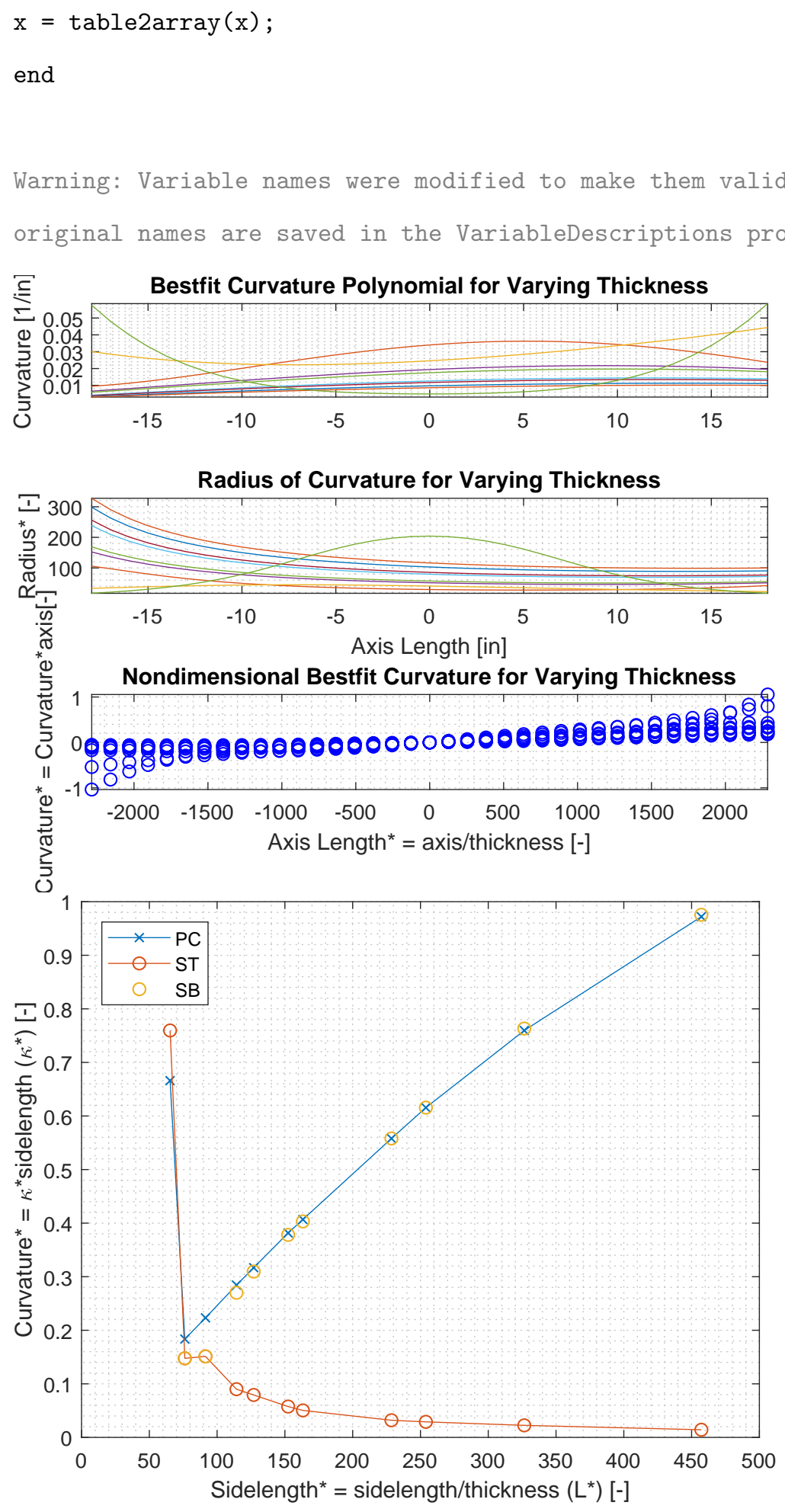


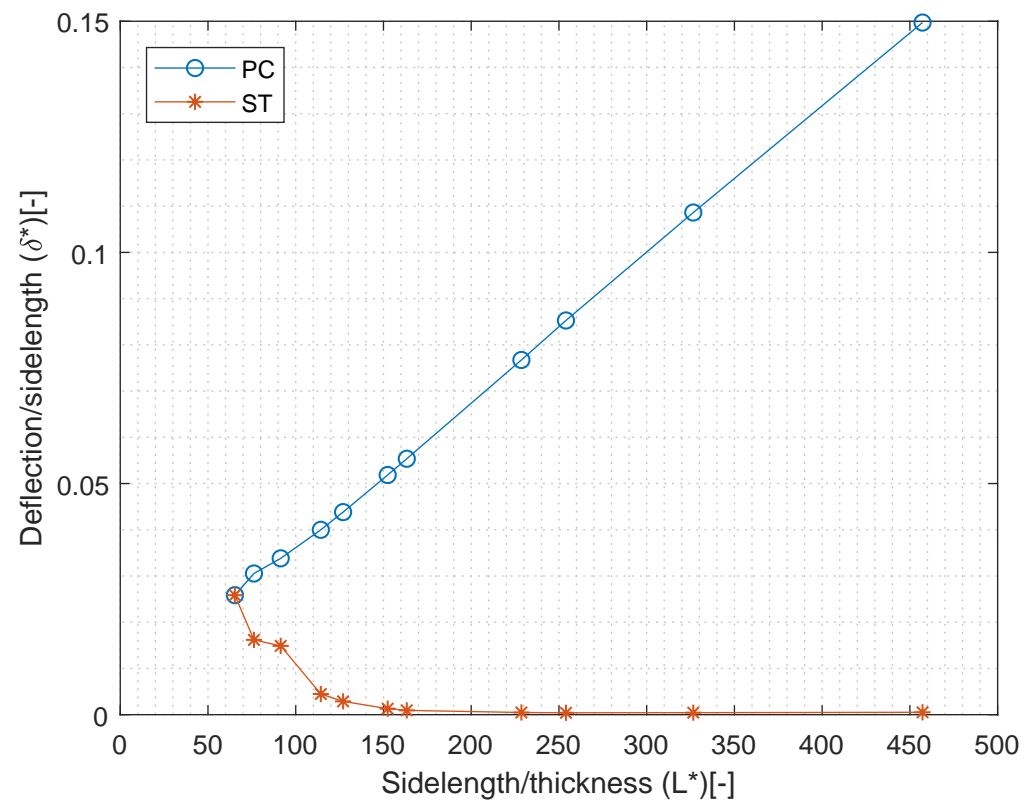

Out-of-Plane Plotter

$\%$ Christopher Knippenberg

$\%$ Out of plane deformation plot

$\%$ Uses equation obtained from Approximations (i.e. Hyer, Mattioni)

$\%$ to give a visual representation of the out of plane deformation

$\%$ of a cross-ply asymmetric composite plate

$\%$ coeffificent values are determined from approximation models i.e.

$\%$ Hyer

clear

clc

close all;

$\%$------------ INPUT PARAMETERS

$\%$ sidelength

$\mathrm{L}=0.914 ; \%$ meters $0.3048 \mathrm{~m}=12 " 0.914 \mathrm{~m}=36 "$ 


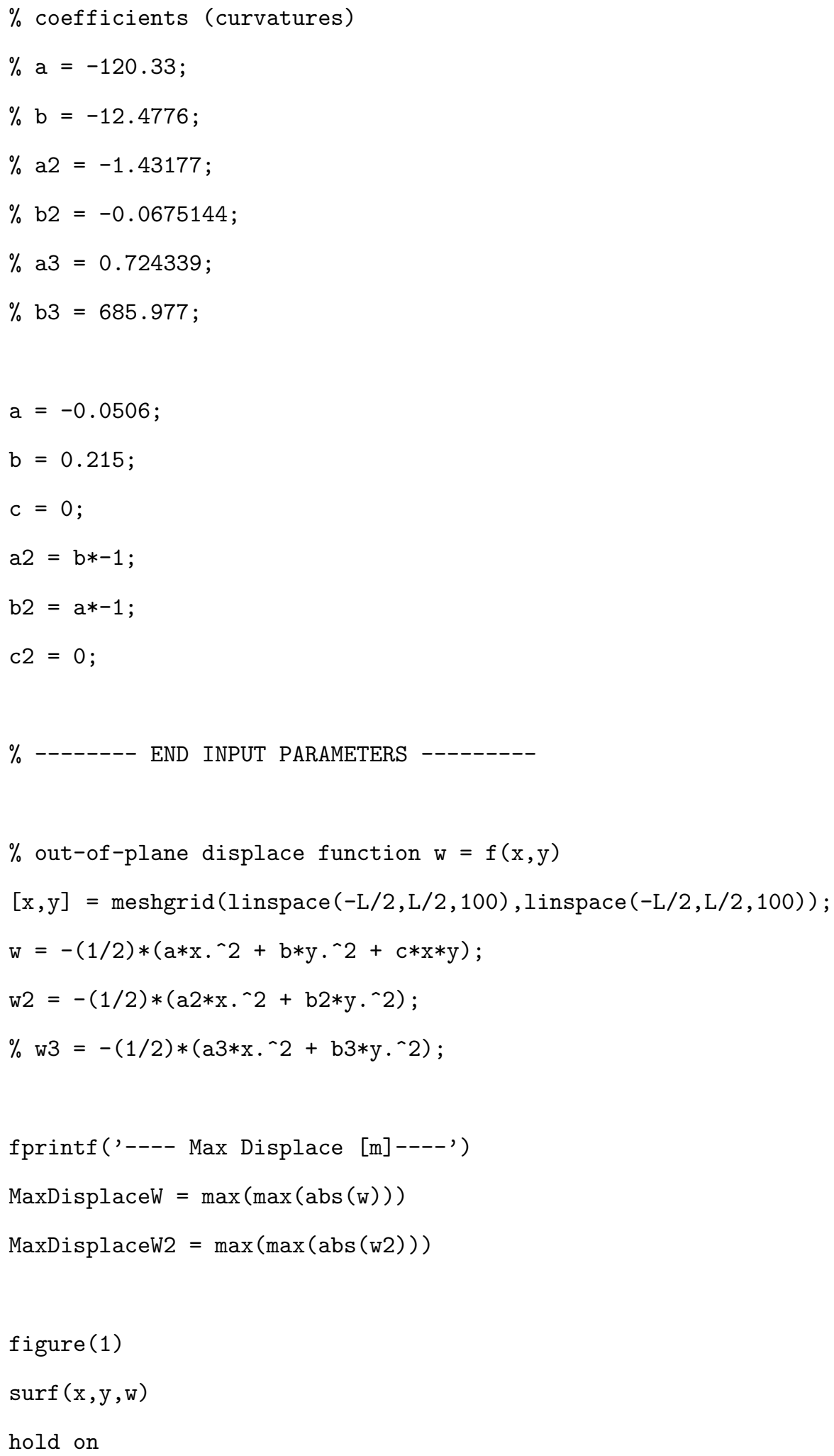



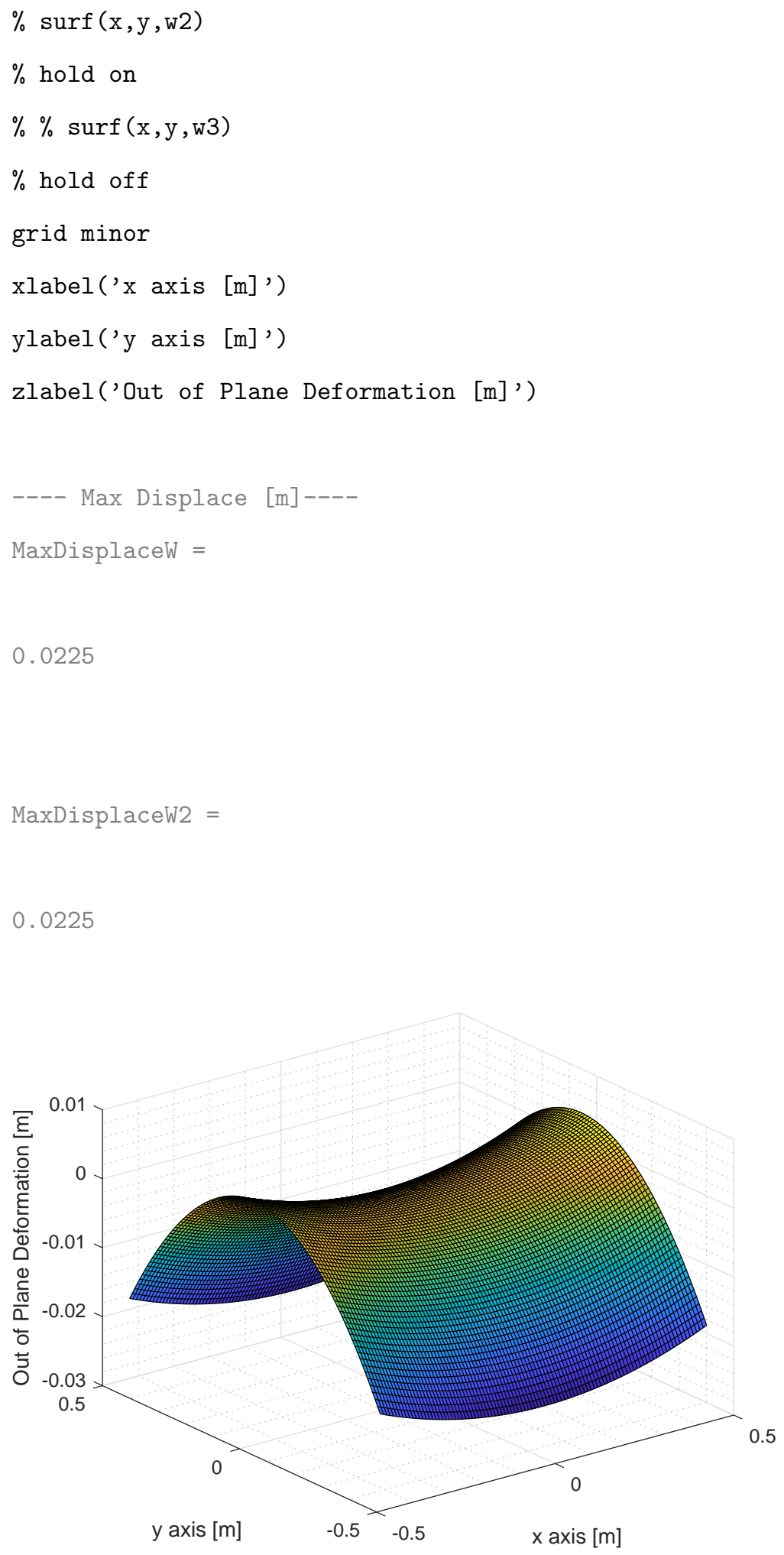


\section{Bibliography}

[1] Donald J. Leo. Engineering Analysis of Smart Material Systems. Wiley, 2007.

[2] Guy Maximilian Kemmann. An Experimental Investigation of Combined Symmetric Asymmetric Composite Laminates. Ms thesis, Clemson University, Clemson, SC, December 2018.

[3] Stephen Daynes and Paul M. Weaver. Review of shape-morphing automobile structures: concepts and outlook. Journal of Automotive Engineering, 227(11):1603-1622, June 2013.

[4] Michael W. Hyer. Calculations of the room-temperature shapes of unsymmetric laminates. Journal of Composite Materials, 15(4):296-310, 1981.

[5] P.M. Weaver F. Mattioni and M.I. Freswell. Multistable composite plates with piecewise variation of lay-up in the planform. International Journal of Solids and Structures, 46(1), January 2009.

[6] Robert M. Jones. Mechanics of Composite Materials. Taylor and Francis, Philadelphia, PA, 1999.

[7] Subramanian Annamalai. Design of Bistable Composite Laminates for Shape Morphing Applications. MS Thesis, Clemson University, Clemson, SC, August 2016.

[8] Serkan Ozbay Samer Tawfik, Xinyuan Tan and Erian Armanios. Anticlastic stabiliity modeling for cross-ply composites. Journal of Composite Materials, 41, November 2007.

[9] Salil Abhijit Phatak. Defining Design Parameters for Bistable Composite Laminates. Ms thesis, Clemson University, Clemson, SC, May 2019.

[10] A. T. Nettles. Basic Mechanics of Laminated Composite Plates. NASA, 1994.

[11] J.N. Reddy. Mechanics of Laminated Plates and Shells: Theory and Analysis. CRC Press, Boca Raton, Florida, 2004.

[12] R.A. Schapery. Thermal expansion coefficients of composite materials based on energy principles. Journal of Composite Materials, 2(3):380-404, 1968.

[13] Guy Kemmann and Oliver Myers. An experimental investigation of combined symmetricasymmetric composite laminates. Journal of Composites Science, 3:71, 072019.

[14] Samir A. Emam and Daniel J. Inman. A review on bistable composite laminates for morphing and energy harvesting. Applied Mechanics Review, 67(6), 122015.

[15] P. C. Bhavani M. W. Hyer. Suppression of anticlastic curvature in isotropic and composite plate. International Journal of Solid Structures, 20(6):553-570, 1984.

[16] A. Hamamoto and M. W. Hyer. Non-linear temperature-curvature relationships for unsymmetric graphite-epoxy lamiates. International Journal of Solids Structures, 23(7):919-935, 1987. 
[17] Christopher J. Brampton et. al. Sensitivity of bistable laminates to uncertainties in material properties, geometry, and environmental conditions", journal $=$ composite Structures, volume $=102$, year $=2013$, pages $=276-286$, issn $=0263-8223$, doi $=$ https://doi.org/10.1016/j.compstruct.2013.03.005, url $=$ http://www.sciencedirect.com/science/article/pii/S0263822313001268.

[18] C. R. Bowen D. N. Betts, H. A. Kim. Modeling and optimization of bistable composite laminates for piezoelectric actuation. Composite Structures, 22(18):2181-2191, 2011.

[19] Marie-Laurie Dano and Michael W. Hyer. Thermally-induced deformation behavior of unsymmetric laminates. International Journal of Solids and Structures, 35(17), June 1998.

[20] International Symposium on Composite Materials and Structures. alculation of the RoomTemperature Shapes of Unsymmetric Laminates, Lancater, PA, 6 1986. Technomic Publishing.

[21] Samer A. Tawfik. Stability and Morphing Characteristics of Bistable Composite Laminates. $\mathrm{PhD}$ thesis, Georgia Institute of Technology, 2008.

[22] W. J. Jun and C. S. Hong. Cured shapes of unsymmetric laminates with arbitrary lay-up angles. Journal of Reinforced Plastic Composites, 11(12):1352-1366, 1992.

[23] L. Warnet L. J. B. Peeters, P. C. Powell. Terhamlly induced shapes of unsymmetric laminates. Journal of Composite Materials, 30(5):603-626, 1996.

[24] M.L. Dano and Michael W. Hyer. Snap-through of unsymmetric fiber-reinforced composite laminates. International Journal of Solids and Structures, 39(1), January 2002.

[25] D. Avitabile A. Pirrera and P.M. Weaver. Bistable plates for morphing structures: A refined analytical approach with high-order polynomials. International Journal of Solids and Structures, 47(25-26), December 2010.

[26] A. L. T. Salo H. A. Kim A. Ive P. F. Giddings, C. R. Bowen. Bistable composite laminates: Efffects of laminate composition on cured shape and response to thermal load. 92(9):2220-2225, 2010.

[27] Marie-Laurie Dano and Michael W. Hyer. The response of unsymmetric laminates to simple applied forces. Mechanics of Composite Materials and Structures, 3(1):65-80, 1996.

[28] M. Schlecht and K. Schulte. Advanced calculation of the room-temperature shapes of unsymmetric laminates. Journal of Composite Materials, 33(16):1472-1490, 1999.

[29] Cezar G. Diaconu, Paul M. Weaver, and Andres F. Arrieta. Dynamic analysis of bi-stable composite plates. Journal of Sound and Vibration, 322(4):987 - 1004, 2009.

[30] I. Adarraga M.A. Cantera, J.M. Romera and F. Mujika. Modelling and testing of the snapthrough process of bi-stable cross-ply composites. Composite Structures, 120:41-52, October 2014.

[31] M.R. Schultz. Use of Piezoelectric Actuators to Effect Snap-Through Behavior of Unsymmetric Composite Laminates. Phd. dissertation, Virgina Polytechnic Institute and State University, Blacksburg, VA, 2003.

[32] The Application of Thermally Induced Mutlistable Composites to Morphing Aircraft Structures, volume 6930 of SPIE 6930, Industrial and Commercial Applications of Smart Structures Technologies, 2008.

[33] M. R. Schultz. A concept for airfoil-like active bistable twisting structures. 2008. 
[34] P. M. Weaver S. Daynes and K.D. Potter. Aeroelastic study of bistable composite airfoils. Journal of Aircraft, 46(6):2169.

[35] A. Erturk A. F. Arrieta, P. Hagedorn and D. J. Inman. A piezoelectric bistable plate for nonlinear broadband energy harvesting. Applied Phsyics Letters, 97(10), 2010.

[36] H. A. Kim D. N. Betts and C. R. Bowen.

[37] Xavier Lachenal, Stephen Daynes, and Paul Weaver. Review of morphing concepts and materials for wind turbine blade applications. Wind Energy, 16:283-307, 032013.

[38] J. C. Leger. Menger curvature and rectifiability. Annals of Mathematics, 149(3):831-869, 1999.

[39] Gjermund Maesel Kolvik. Higher Order Shear Deformation Plate Theory, May 2012.

[40] Inc. Gourgeon Brothers. Vacuum bagging techniques, 7th ed. https://www.westsystem.com/wp-content/uploads/VacuumBag-7th-Ed.pdf, April 2010. 